Günter Kimmeskamp

\title{
Die Rollenbeurteilung von Handelsvertretungen
}




\section{Günter Kimmeskamp}

\section{Die Rollenbeurteilung von Handelsvertretungen}

Im Spannungsfeld von Industrie und Handel vermitteln Handelsvertretungen nahezu die Hälfte des Inlandsumsatzes der deutschen Industrie. In jüngerer Zeit wird der Aufgabenbereich von Handelsvertretungen unter Marketingaspekten verstärkt diskutiert. Die vorliegende Arbeit strukturiert das Dienstleistungsangebot von Handelsvertretungen und arbeitet wechselseitige Meinungen und insbesondere Anforderungen der Marktpartner an das Dienstleistungsangebot von Handelsvertretungen heraus. Die Aussagen basieren auf den Resultaten einer repräsentativen Befragung bei Industrieunternehmen, Handelsbetrieben und Handelsvertretungen.

Günter Kimmeskamp, geboren 1951, studierte Betriebswirtschaftslehre an der Universität Münster von 1972 bis 1977. Examen als Dipl.-Kfm. 1977 in Münster. Seither Assistent am Institut für Marketing der Westfälischen Wilhelms-Universität Münster. 
Die Rollenbeurteilung von Handelsvertretungen 


\section{Schriffen zum Marketine}

Herausgegeben von Prof.Dr. Heribert Meffert

\section{Band 3}

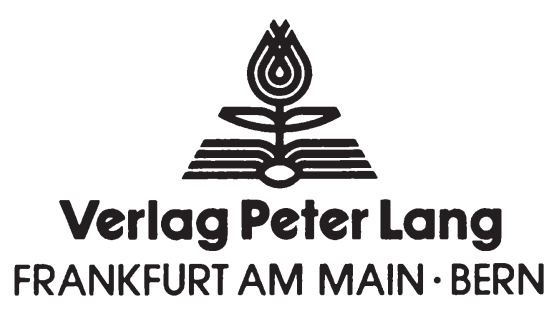




\section{Günter Kimmeskamp}

\section{Die Rollenbeurteilung von Handelsvertretungen}

Eine empirische Untersuchung zur Einschätzung des Dienstleistungsangebotes durch Industrie und Handel

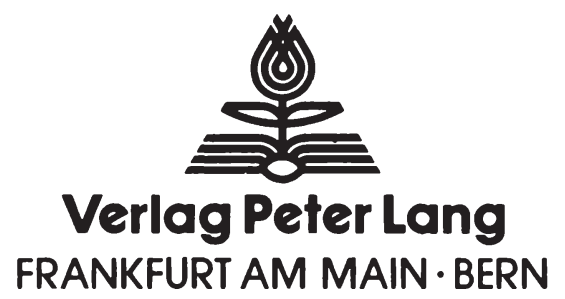


CIP-Kurztitelaufnahme der Deutschen Bibliothek

\section{Kimmeskamp, Günter:}

Die Rollenbeurteilung von Handelsvertretungen :

e. empir. Unters. zur Einschätzung d. Dienst= leistungsangebotes durch Industrie u. Handel /

Günter Kimmeskamp. - Frankfurt am Main ; Bern : Lang, 1982.

(Schriften zum Marketing ; Bd. 3)

ISBN 3-8204-5700-3

NE: GT

Open Access: The online version of this publication is published on www.peterlang.com and www.econstor.eu under the international Creative Commons License CC-BY 4.0. Learn more on how you can use and share this work: http://creativecommons. org/licenses/by/4.0.

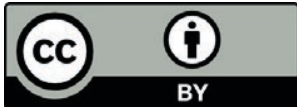

This book is available Open Access thanks to the kind support of ZBW - Leibniz-Informationszentrum Wirtschaft.

\section{ISBN 3-8204-5700-3 \\ ISBN 978-3-631-75039-1 (eBook) \\ (C) Verlag Peter Lang GmbH, Frankfurt am Main 1982 \\ Alle Rechte vorbehalten.}

Nachdruck oder Vervielfältigung, auch auszugsweise, in allen Formen wie Mikrofilm, Xerographie, Mikrofiche, Mikrocard, Offset verboten.

Druck und Bindung: fotokop wilhelm weihert KG, darmstadt 


\section{Vorwort}

Handelsvertretungen bilden in weiten Bereichen der Wirtschaft ein wichtiges Bindeglied zwischen produzierenden Unternehmen und ihren Abnehmern. Gerade in jüngerer Zeit wird vielfach von einer Renaissance des Handelsvertreters gesprochen. Demgegenüber finden handelsvertreterspezifische Marketingprobleme nur eine geringe Berücksichtigung in der neueren absatzwirtschaftlichen Literatur.

Zielsetzung der vorliegenden Arbeit ist eine marketingorientierte Neubestimmung des Tätigkeitsbereichs von Handelsvertretungen. Damit soll die Lücke zwischen der Einbettung von Handelsvertretungen in das klassische Herstellermarketing auf der einen seite und der wachsenden Betonung der Eigenständigkeit von Handelsvertretungen als Dienstleistungsunternehmen auf der anderen Seite geschlossen werden. Auf der Grundlage rollentheoretischer Utberlegungen werden dazu die Resultate einer Befragung bei Industrieunternehmen, Handelsbetrieben und Handelsvertretungen herangezogen. Diese Erhebungen ermöglichen Einblicke in die wechselseitigen Beurteilungen und Anforderungen der verschiedenen Marktpartner und bilden die Grundlage für die Konkretisierung eines Handelsvertreter-Marketing.

Die Abfassung dieser primär empirisch ausgerichteten Arbeit war nur mit vielfältiger Unterstützung verschiedener Personen möglich. Mein besonderer Dank gilt an dieser Stelle meinem akademischen Lehrer, Herrn Professor Dr. Heribert Meffert für die Anregung der Themenstellung sowie umfassende Förderung bei der Anfertigung der Arbeit.

Konzeptionelle Vorbereitung sowie Durchführung der empirischen Untersuchung erfolgten mit Unterstützung des For- 
schungsverbandes für den Handelsvertreter- und Handelsmaklerberuf in Köln. Vorstand und Geschäftsführung standen darüber hinaus jederzeit als kompetente Gesprächspartner bereit. Dafür danke ich insbesondere Herrn Dr. H.J. Bless, Herrn Dipl.-Volksw. H. Voss, Herrn Dipl.-Volksw. H. Quitzau sowie den Herren des Kuratoriums des Forschungsverbandes.

Mein Dank gilt ferner allen Kolleginnen und Kollegen am Institut für Marketing für ihre freundliche Unterstützung. Dies gilt in besonderem Maße Frau Dr. Simone PfeifferBohnenkamp, Herrn Dr. Jürgen Althans und Herrn Dr. Werner Kook für ihre ständige Bereitschaft zur kritischen Diskussion. Frau M. Bürger danke ich für die reibungslose Abwicklung der Schreibarbeiten. Schlieblich gebührt meiner Frau ein herzliches Dankeschön für das entgegengebrachte Verständnis sowie die vielfältigen Entlastungen während der Abfassung dieser Arbeit. 


\section{Vorwort des Herausgebers}

Funktionen und Leistungen von Handelsvertretungen als traditionell bedeutsamem Bereich der Absatzwirtschaft fanden in der neueren Marketingliteratur vergleichsweise wenig Beachtung. Die Diskussion erfolgte zudem schwerpunktmäBig aus der sicht von Herstellerunternehmen. In jüngerer zeit hat sich die Diskussion im Hinblick auf die Eigenständigkeit von Handelsvertretungen als Dienstleistungsunternehmen verlagert. Die vorliegende Arbeit hat zum ziel, die erkennbare Lücke zwischen der Einbettung einer Handelsvertretung in die klassische Absatzwirtschaft einerseits und der Selbständigkeit dieses Dienstleistungsunternehmens andererseits durch eine marketing-orientierte Neubestimmung des Tätigkeitsbereichs von Handelsvertretungen zu schließen.

Ausgehend von der dualen Stellung der Handelsvertretung im System des Marketing wird eine rollenorientierte Analyse der Dienstleistungen bzw. des Marketing von Handelsvertretungen vorgenommen. Dabei wird sowohl die Ubertragbarkeit rollentheoretischer Uberlegungen auf Problembereiche des Handelsvertreter-Marketing geprüt als auch eine operationale Umsetzung im Hinblick auf eine umfassende empirische Analyse der Rolle von Handelsvertretungen durchgeführt.

Einige Aspekte der Arbeit verdienen eine besondere Hervorhebung. Dies gilt einmal hinsichtlich der Entwicklung des innovativen rollentheoretischen Bezugsrahmens und der daraus abgeleiteten Hypothesen zum Handelsvertretermarketing. Zum anderen greift der Verfasser den neuen Gedanken einer dualistischen Interpretation des Handelsvertreters auf und entwickelt diesen auf empirischer Grundlage weiter. Schließlich dokumentiert die Arbeit eine umfassende empirische Uberprüfung des Hypothesengerüsts auf repräsentativer Grundlage. In einer sorgfältig angelegten Befragung wurden Handelsvertretungen, Hersteller und 
Kunden im Hinblick auf die relevanten empirischen Zusammenhänge analysiert.

Insgesamt bringt die theoriegeleitete empirische Arbeit einen beachtlichen Fortschritt der Forschung im Bereich des vertikalen Marketing und eröffnet der Diskussion über ein wirksames Handelsvertretermarketing in der Praxis neue Perspektiven.

Münster, im August 1981 Prof. Dr. Heribert Meffert 


\section{INHALTSVERZEICHNIS}

Seite

A. Handelsvertreter-Marketing im Spannungsfeld von Industrie und Handel

1. Entwicklung des Handelsvertreter-Marketing

2. Duale Stellung der Handelsvertretung im vertikalen Marketing

3. Ziel und Gang der Untersuchung

B. Verhaltenswissenschaftlicher Bezugsrahmen eines Handelsvertreter-Marketing

1. Rollenanalyse als Grundlage eines dualistischen Handelsvertreter-Marketing

1.1 Begriffliche Grundlagen der Rollenanalyse 16

1.2 Rollenkomponenten im Handelsvertreter-Marketing 24

1.21 Rollenausübung von Handelsvertretungen 24

1.22 Rollenerwartungen an Handelsvertretungen 27

1.23 Rollenerfüllung von Handelsvertretungen 29

1.3 Rollenbeziehungen im Handelsvertreter-Marketing 31

1. 31 Integration der Rollenkomponenten (Rollenmodell des Handelsvertreter-Marketing)

1. 32 Horizontale Rollenbeziehungen

1.33 Vertikale Rollenbeziehungen

2. Ansatzpunkte zur Erfassung von Rollen und Rollenbeziehungen im Handelsvertreter-Marketing

2. 1 Dienstleistungen von Handelsvertretungen als rollenprägende Merkmale

2.11 Die Eignung von Dienstleistungen zur Erfassung von Rollen

2.12 Operationalisierung von Dienstleistungen für eine Rollenanalyse 
Seite

2.13 Kennzeichnung der Dienstleistungsbereiche von Handelsvertretungen

2.2 Bestimmungsfaktoren von Rollen und Rollenbeziehungen

2.21 Merkmale der Handelsvertretungen

2.22 Merkmale der vertretenen Unternehmen

2.23 Merkmale des Kundenmarktes

2.3 Zusammenführung von Rollen und Bestimmungsfaktoren im Handelsvertreter-Marketing

3. Hypothesen zur Rollenanalyse im HandelsvertreterMarketing

3. 1 Hypothetische Annahmen einer rollenbezogenen Gruppierung der Dienstleistungsmerkmale

3.2 Hypothetische Annahmen zu einzelnen Rollenkomponenten

3. 3 Hypothetische Annahmen zu Rollenbeziehungen

3.4 Hypothetische Annahmen zur rollenbezogenen Segmentierung der Marktpartner

C. Empirische Untersuchung zum Marketing von Handelsvertretungen

1. Untersuchungsdesign und Stichprobenstruktur

2. Ermittlung der zentralen Dienstleistungsbereiche von Handelsvertretungen

2.1 Komponenten der Rollenausübung von Handelsvertretungen

2. 2 Komponenten von Rollenerfüllung und-erwartung aus der Sicht der Marktpartner

2.21 Struktur der Rollenerfüllung und -erwartung im Urteil der vertretenen Unternehmen

2.22 Struktur der Rollenerfüllung und -erwartung im Urteil der Kunden 
Seite

2.3 Gegenüberstellung der ermittelten Dienstleistungsstrukturen

3. Analyse der Rollenkomponenten im HandelsvertreterMarketing

3. 1 Rollenausübung von Handelsvertretungen

3. 11 Bestimmungsfaktoren der Rollenausübung im Überblick

3.12 Bestimmungsfaktoren der Rollenausübung einzelner Dienstleistungsbereiche

3.121 Informationsaustausch 96

3.122 Innerbetriebliche Leistungsfähigkeit 98

3. 123 Preisgestaltung und Leistungsvergütung 100

3.124 Vertretungsprogramm und Produktanregungen

102

3.13 Zusammenfassende Hypothesenbeurteilung 106

3.2 Rollenerfüllung und -erwartung im Urteil der vertretenen Unternehmen

108

3. 21 Bestimmungsfaktoren von Rollenerfüllung und -erwartung im Uberblick

3. 22 Bestimmungsfaktoren von Rollenerfüllung und-erwartung in einzelnen Dienstleistungsbereichen

3.221 Informationsaustausch

3.222 Kundenbetreuung

3. 223 Produktanregungen

3.224 Preisgestaltung und Leistungsvergütung 125

3.225 Ausstattung der Handelsvertretung

3.23 Zusammenfassende Hypothesenbeurteilung

3. 3 Rollenerfüllung und -erwartung im Urteil der Abnehmer

3. 31 Bestimmungs faktoren von Rollenerfüllung und -erwartung im uberblick

3. 32 Bestimmungsfaktoren von Rollenerfüllung und-erwartung in einzelnen Dienstleistungsbereichen

3.321 Preisgestaltung

3. 322 Produktanregungen 
Seite

3. 323 Innerbetriebliche Leistungsfähigkeit 153

3.324 Vertretungeprogramm 156

3. 33 Zusammenfassende Hypothesenbeurteilung 160

4. Analyse der Rollenbeziehungen im Handelsvertreter- 164 Marketing

4. 1 Horizontale Rollenbeziehungen

4.11 Rollenbeziehungen auf Herstellerebene

4.111 Ausprägungen der Rollenbeziehungen

4.112 Bestimmungsfaktoren der Rollenbeziehungen

4.12 Rollenbeziehungen auf Kundenebene

4.121 Ausprägungen der Rollenbeziehungen

4.122 Bestimmungsfaktoren der Rollenbeziehungen

4.13 Zusammenfassende Hypothesenbeurteilung horizontaler Rollenbeziehungen

4. 2 Vertikale Rollenbeziehungen

4.21 Empirische Grundstruktur von Rollendissens und-defizit

4.22 Rollenbeziehungen zwischen Handelsvertretungen und ihren Marktpartnern in einzelnen Dienstleistungsbereichen

4.221 Beziehungen zwischen Handelsvertretungen und Herstellern

4.222 Beziehungen zwischen Handelsvertretungen und Kunden

4.23 Zusammenfassende Hypothesenbeurteilung vertikaler Rollenbeziehungen

5. Rollenbezogene Segmentierung der Marktpartner als Grundlage eines differenzierten HandelsvertreterMarketing

5. 1 Ansatzpunkte und Vorgehensweise einer psychographischen Segmentierung im Rahmen der Rollenanalyse

5.2 Typologisierung der vertretenen Unternehmen 
Seite

5.22 Inhaltliche und demographische Segmentstruktur auf Herstellerebene

5.221 Segment I: Verkaufsorientierung mit Direktvertrieb

5.222 Segment II: Eingeschränkte Dienstleistungsorientierung mit Reisendenvertrieb

5.223 Segment III: Umfassende Dienstleistungsorientierung mit Eigenverantwortung der Handelsvertreter

5.3 Typologisierung der Abnehmer

5.31 Festlegung der Gruppenanzahl

5.32 Inhaltliche und demographische Segmentstruktur auf Kundenebene

5.321 Segment I: Dienstleistungsorientierte Abnehmer mittlerer Größe

5.322 Segment II: Kleinkunden mit geringer Handelsvertreterorientierung

5.323 Segment III: Preisorientierte Großabnehmer

5.4 Zusammenfassende Hypothesenbeurteilung der rollenbezogenen Segmentierung

D. Implikationen der Untersuchung zum Marketing von Handelsvertretungen

1. Auswirkungen für die weitere Forschung

2. Auswirkungen für das strategische HandelsvertreterMarketing

Anhang I : Tabellen

Anhang II: Fragebogen 


\section{Abbildungsverzeichnis}

Seite

Abb. 1: Tätigkeitsbereiche von Handelsvertretungen

Abb. 2: Dualistische Marketingkonzeption von Handelsvertretungen

Abb. 3: Grundmodell der allgemeinen Rollenanalyse

Abb. 4: Rollenmodell des Handelsvertreter-Marketing

Abb. 5: Struktur des Dienstleistungsangebotes von Handelsvertretungen (mit Beispielen)

Abb. 6: Bestimmungsfaktoren der Rollenausübung von Handelsvertretungen

$\mathrm{Abb}$. 7: Bestimmungsfaktoren von Rollenerwartung und -erfüllung bei den vertretenen Unternehmen

Abb. 8: Bestimmungsfaktoren von Rollenerwartung und -erfüllung beim Kundenmarkt

Abb. 9: Grundstruktur und Hypothesengerüst einer Rollenanalyse im Handelsvertreter-Marketing

Abb. 10: Stichprobenumfang und Verteilung auf Wirtschaftsbereiche

Abb. 11: Struktur des Kundensegmentes nach Betriebsformen

Abb. 12: Struktur der Rollenausübung von Handelsvertretungen

Abb. 13: Struktur der Rollenerfüllung von Handelsvertretungen im Urteil der vertretenen Unternehmen 
Abb. 14: Struktur der Rollenerwartung an Handelsvertretungen durch die vertretenen Unternehmen

Abb. 15: Struktur der Rollenerfüllung von Handelsvertretungen im Urteil der Kunden

Abb. 16: Struktur der Rollenerwartung an Handelsvertretungen durch die Kunden

Abb. 17: Bestimmungsfaktoren der Rollenausübung von Handelsvertretungen im tberblick

Abb. 18: Unternehmens- und personenbezogene Bestimmungsfaktoren der Rollenausübung beim Informationsaustausch

Abb. 19: Kundenbezogene Bestimmungsfaktoren der Rollenausübung in der innerbetrieblichen Leistungsfähigkeit

Abb. 20: GröBenbezogene Bestimmungsfaktoren der Rollenausuibung in der innerbetrieblichen Leistungsfähigkeit

Abb. 21: Unternehmens- und personenbezogene Bestimmungsfaktoren der Rollenausübung in der Preisgestaltung

Abb. 22: Unternehmens- und personenbezogene Bestimmungsfaktoren der Rollenausuibung. in der Leistungsvergütung

Abb. 23: Unternehmensbezogene Bestimmungsfaktoren der Rollenausübung beim Vertretungsprogramm

Abb. 24: Kundenbezogene Bestimmungsfaktoren der Rollenausuibung bei den Produktanregungen 
Abb. 25: Zusammenfassende Hypothesenbeurteilung zur Rollenausübung von Handelsvertretungen

Abb. 26: Bestimmungsfaktoren der Rollenerfüllung von Handelsvertretungen aus der Sicht der Hersteller im Úberblick

Abb. 27: Bestimmungsfaktoren der Rollenerwartung an Handelsvertretungen durch die Hersteller im Uberblick

Abb. 28: Unternehmensbezogene Bestimmungsfaktoren der Rollenerwartung durch die Hersteller beim Informationsaustausch

Abb. 29: Zusammenhänge zwischen der Vertriebsstruktur und der Rollenerfüllung hinsichtlich der Erweiterung des Kundenkreises

Abb. 3o: Vertriebsbezogene Bestimmungsfaktoren der Rollenerfüllung von Handelsvertretungen bei den Produktanregungen

Abb. 31: Zusammenhänge zwischen den Herstellererwartungen für Neuproduktanregungen und einzelnen Wirtschaftsbereichen

Abb. 32: Zusammenhänge zwischen der Vertriebsstruktur und der Rollenerfüllung hinsichtlich des preispolitischen Handlungsspielraumes

Abb. 33: Personenbezogene Bestimmungsfaktoren der Rollenerwartung an Handelsvertretungen bei der Preis- und Konditionengestaltung

Abb. 34: Zusammenhänge zwischen den Rollenerwartungen im Bereich der Leistungsvergütung und der Branchenstruktur

$\mathrm{Abb}$. 35: Unternehmensbezogene Bestimmungsfaktoren der Rollenerfüllung und -erwartung bei der Ausstattung der Handelsvertretung 
Abb. 36: Zusammenhänge zwischen der Beurteilung der Rollenerfüllung hinsichtlich der Ausstattung von Handelsvertretungen und der Branchenstruktur

Abb. 37: Zusammenfassende Hypothesenbeurteilung zur Rollenerfüllung und Rollenerwartung aus der Sicht der vertretenen Unternehmen

Abb. 38: Bestimmungsfaktoren der Rollenerfüllung von Handelsvertretungen aus der Sicht der Kunden im Uberblick

Abb. 39: Bestimmungsfaktoren der Rollenerwartung an Handelsvertretungen durch die Kunden im Uberblick

$\mathrm{Abb}$. 40: Unternehmens- und personenbezogene Bestimmungsfaktoren der Rollenerfüllung von Handelsvertretungen bei der Preisgestaltung

Abb. 41: Zusammenhänge zwischen der Beurteilung des preispolitischen Handlungsspielraumes und unterschiedlichen Betriebsformen auf Kundenebene

Abb. 42: Einkaufsbezogene Bestimmungsfaktoren der Rollenerfüllung und -erwartung der Kunden bei den Produktanregungen

Abb. 43: Einkaufsbezogene Bestimmungsfaktoren der Rollenerfüllung und -erwartung der Kunden bei der innerbetrieblichen Leistungsfähigkeit

Abb. 44: Einkaufsbezogene Bestimmungsfaktoren der Rollenerfüllung aus Kundensicht beim Vertretungsprogramm 
Abb. 45: Unternehmensbezogene Bestimmungsfaktoren der Rollenerwartung aus Kundensicht beim Vertretungsprogramm

Abb. 46: Zusammenfassende Hypothesenbeurteilung zur Rollenerfüllung und Rollenerwartung aus der sicht der Abnehmer

Abb. 47: Ausprägungen des Grades der Rollenerfüllung von Handelsvertretungen aus Herstellersicht

Abb. 48: Grad der Rollenerfüllung in einzelnen Dienstleistungsbereichen aus Herstellersicht

Abb. 49: Bestimmungsfaktoren horizontaler Rollenbeziehungen auf Herstellerebene

Abb. 50: Ausprägungen des Grades der Rollenerfüllung aus Kundensicht

Abb. 51: Grad der Rollenerfüllung in einzelnen Dienstleistungsbereichen aus Kundensicht

Abb. 52: Bestimmungsfaktoren horizontaler Rollenbeziehungen auf Kundenebene

Abb. 53: Zusammenfassende Hypothesenbeurteilung horizontaler Rollenbeziehungen auf Herstellerund Kundenebene

Abb. 54: Rollenbeziehungen zwischen Handelsvertretungen und vertretenen Unternehmen

Abb. 55: Rollenbeziehungen zwischen Handelsvertretungen und Kunden

Abb. 56: Zusammenfassende Hypothesenbeurteilung vertikaler Rollenbeziehungen 
Abb. 57: Segmentaufteilung des Herstellermarktes

Abb. 58: Segmentspezifische Ausprägungen von Rollenkomponenten auf Herstellerebene Segment I: Verkaufsorientierung mit Direktvertrieb

Abb. 59: Demographische Struktur von Herstellersegment I

Abb. 6o: Segmentspezifische Ausprägungen von Rollenkomponenten auf Herstellerebene Segment II: Eingeschränkte Dienstleistungsorientierung mit Reisendenvertrieb

Abb. 61: Demographische Struktur von Herstellersegment II

Abb. 62: Segmentspezifische Ausprägungen von Rollenkomponenten auf Herstellerebene Segment III: Umfassende Dienstleistungsorientierung mit Eigenverantwortung der Handelsvertreter

Abb. 63: Demographische Struktur von Herstellersegment III

Abb. 64: Segmentaufteilung des Kundenmarktes

Abb. 65: Segmentspezifische Ausprägungen von Rollenkomponenten auf Kundenebene Segment I: Dienstleistungsorientierte Abnehmer mittlerer Größe

Abb. 66: Demographische Struktur von Kundensegment I 240

Abb. 67: Segmentspezifische Ausprägungen von Rollenkomponenten auf Kundenebene Segment II: Kleinkunden mit geringer Handelsvertreterorientierung 
Seite

Abb. 68: Demographische Struktur von Kundensegment II

Abb. 69: Segmentspezifische Ausprägungen von Rollenkomponenten auf Kundenebene -

Segment III: Preisorientierte Großabnehmer

Abb. 70: Demographische Struktur von Kundensegment II.I

Abb. 71: Konzeption des integrierten Handelsvertreter-Marketing 


\section{Abkürzungsverzeichnis}

a.a.O.

$\mathrm{Abb}$.

AJS

AMA

Art.

ASM

asw

ASQ

Aufl.

Bd.

BJS

bzgl.

bzw.

DBW

DM

ders.

d.h.

Diss.

et al.

FAZ

f. (f).

$\mathrm{FfH}$

$\mathrm{H}$

HdA

HGB

Hrsg.

HV

$\mathrm{HV}+\mathrm{HM}$

i.d. R.

insbes.

IMM

IUA am angegebenen ort

Abbildung

American Journal of Sociology

American Marketing Association

Artikel

Agency Sales Magazine

Absatzwirtschaft

Administrative Science Quarterly

Auflage

Band

British Journal of Sociology

bezüglich

beziehungsweise

Die Betriebswirtschaft

Deutsche Mark

derselbe

das heiBt

Dissertation

et alteri

Frankfurter Allgemeine Zeitung

folgend (e)

Forschungsstelle für den Handel

Hypothese

Handwörterbuch der Absatzwirtschaft

Handelsgesetzbuch

Herausgeber

Handelsvertreter/-ung

Der Handelsvertreter und Handelsmakler

in der Regel

insbesondere

Industrial Marketing Management

Interview und Analyse 


\begin{tabular}{|c|c|}
\hline JAVF & $\begin{array}{l}\text { Jahrbuch der Absatz- und Verbrauchs- } \\
\text { forschung }\end{array}$ \\
\hline JoB & Journal of Business \\
\hline JoM & Journal of Marketing \\
\hline JOMR & Journal of Marketing Research \\
\hline JoSBM & Journal of Small Business Management \\
\hline Kap. & Kapitel \\
\hline KZfSS & $\begin{array}{l}\text { Kölner Zeitschrift für Soziologie } \\
\text { und Sozialpsychologie }\end{array}$ \\
\hline LZ & Lebensmittel-Zeitung \\
\hline MA & Markenartikel \\
\hline MS & Management Science \\
\hline $\mathrm{n}$ & Stichprobenumfang \\
\hline No. & Number \\
\hline $\mathrm{Nr}$ & Nummer \\
\hline 0.0 & ohne Ort \\
\hline o.v. & ohne Verfasser \\
\hline RKW & $\begin{array}{l}\text { Rationalisierungs-Kuratorium der } \\
\text { Deutschen Wirtschaft }\end{array}$ \\
\hline S. & Seite \\
\hline sog. & sogenannt \\
\hline Sp. & Spalte \\
\hline SPSS & $\begin{array}{l}\text { Statistical Package for the Social } \\
\text { Sciences }\end{array}$ \\
\hline Tab. & Tabelle \\
\hline u.a. & und andere; unter anderem \\
\hline usw. & und so weiter \\
\hline vgl. & vergleiche \\
\hline Vol. & Volume \\
\hline VuM & Verkauf und Marketing \\
\hline Wist & Wirtschaftswissenschaftliches studium \\
\hline z.B. & zum Beispiel \\
\hline $\mathrm{ZfB}$ & Zeitschrift für Betriebswirtschaft \\
\hline ZfgSt & $\begin{array}{l}\text { Zeitschrift für die gesamte staats- } \\
\text { wissenschaft }\end{array}$ \\
\hline
\end{tabular}


A. Handelsvertreter-Marketing im Spannungsfeld von Industrie und Handel

1. Entwicklung des Handelsvertreter-Marketing

Der Grundgedanke des Marketing hat sich mit dem Wandel von einer produktions- und verkaufsorientierten $z u$ einer kundenbezogenen Denkweise als marktorientierte Führung des gesamten Unternehmens vielfach etabliert ${ }^{1}$. Ausgehend von seiner klassischen Domäne im Konsumgüterbereich setzte sich die bewußte Ausrichtung an den wünschen, Problemen und Bedürfnissen der Kunden allmählich in industriellen wirtschaftsbereichen und im Dienstleistungssektor durch ${ }^{2}$. "Im Lichte dieser Entwicklung erstaunt die Tatsache, daß traditionell bedeutsame Bereiche der Absatzwirtschaft scheinbar übergangen wurden. Hierzu zählt u.a. der Handelsvertreter" ${ }^{3}$.

$1 \mathrm{zu}$ den zentralen Merkmalen des Marketing vgl. Meffert, H., Art.: Marketing, in Management-Enzyklopädie, Bd. 4, München 1971, S. 383 ff.; Kotler, P., Marketing-Management. Analyse, Planung und Kontrolle, Stuttgart 1974, S. $3 \mathrm{ff} . ;$ Ahlert, D., Grundzüge des Marketing, Düsseldorf 1980, S. $2 \mathrm{f}$..

2 Vgl. Meffert, H., Perspektiven des Marketing in den 8oer Jahren - ein Uberblick des Herausgebers, in: Marketing im Wandel, Hrsg.: H. Meffert, Wiesbaden 1980, S. 3; Die Verbreitung des Marketingdenkens beschränkt sich dabei nicht auf Großbetriebe. Vielmehr haben bereits ca. 12 \& mittelständischer Unternehmen aller Größenklassen (ab 100 bis über 5.000 ) das Marketing als organisatorisch integriertes Managementkonzept realisiert, bei weiteren $13 \%$ steht das Marketing gleichberechtigt neben dem Verkauf. Unterschiede von 8 \& zu 16 \& kennzeichnen dagegen die Diffundierung bei Investitionsgütern im Vergleich $\mathrm{zu} \mathrm{Ge}-$ und Verbrauchsgütern. Vgl. Hartan, M., Marketing- und Vertriebsorganisation in mittelständischen Unternehmen, Teil 1, Eingliederung des Marketing in die Aufbauorganisation der befragten Unternehmen, RKW-Schriftenreihe Marktorientierte Unternehmensführung-Absatzwirtschaft, Frankfurt 1974, S. 8.

3 Meffert, H., Die Handelsvertretung im Spannungsfeld des Marketing-Systems, in: HV + HM 1976, S. 83. 
Eine vergleichsweise stiefmütterliche Behandlung in der neueren Marketingliteratur ${ }^{1}$ steht dabei in offenem Widerspruch zu einer vielfach diskutierten Renaissance des Handelsvertreters ${ }^{2}$. Diese Renaissance bezieht sich dabei weniger auf eine quantitativ meBbare Ausweitung der Handelsvertretertätigkeit ${ }^{3}$, vielmehr werden Chancen und Probleme dieses Absatzmittlers - nicht zuletzt angesichts

1 Vgl. Meffert, H., Die Handelsvertretung ..., a.a.0.; den genannten Sachverhalt belegen die Ergebnisse einer Studie, daß weder in Lehwwerken der allgemeinen Betriebswirtschaftslehre bzw. des Marketing - abgesehen von einer Ausnahme - noch in Zeitschriften eine ausführliche und umfassende Darstellung der Tätigkeit von Handelsvertretungen anzutreffen ist; vgl. Schenk, H.O., Spannagel, R., Wölk, A., Funktionen und Leistungen der Handelsvertretung im Wettbewerb der Vertriebssysteme, Berlin 1974, S. 29 f. bzw. 65 .

2 Vgl. Voss, H., Handelsvertreter oder Reisende - eine Renaissance für Handelsvertreter?, in: Handbuch Marketing, Hrsg.: J. Koinecke, S. $671 \mathrm{ff.;}$ Jeske, J.J., 8er Musterkoffer reicht schon längst nicht. mehr, in: FAZ, Nr. 281 vom 3.12.1977, S. 11; O.V., Ein neues Berufsbild angestrebt, in: Handelsblatt $\mathrm{Nr} .114$ vom 10.7.1978, S. 19; Irgel, L., Wieder im kommen: der Handelsvertreter, in: MJ 1976, S. $323 \mathrm{ff}$.. Diese Entwicklung ist dabei nicht national begrenzt; vgl. auch Kuzela, L., How to work with a Manufacturer's Rep, in: Industry Week 1977, Heft 2, s. $40 \mathrm{ff} . ;$ Agents show their effectiveness in the Automotive Aftermarket, in: ASM 1979, Heft 10, S. 31 .

3 Mit mehr als $350 \mathrm{Mrd}$. DM setzen Handelsvertretungen knapp die Hälfte des Inlandsumsatzes der deutschen Industrie um, vgl. Jeske, J.J., a.a.0., S. 11; 0.V., Handelsvertreter setzen viel um, in: Handelsblatt $\mathrm{Nr} .243$ vom 19.12.1977, S. 11 . 
konjunktureller Entwicklungen - kritisch neu überdacht ${ }^{1}$. Kristallisationspunkt der Diskussion wird zunehmend die Eigenständigkeit von Handelsvertretungen als Dienstleistungsunternehmen ${ }^{2}$.

Die hier erkennbare Lücke zwischen der bisherigen Einbettung der Handelsvertretung in das klassische Marketing auf der einen Seite und der Selbständigkeit dieses Dienstleistungsunternehmens auf der anderen seite regt eine marketingorientierte Neubestimmung des Tätigkeitsbereichs von Handelsvertretungen und ihrer stellung im vertikalen Marketing an.

1 Der Einfluß konjunktureller Entwicklungen auf die Tätigkeit von Handelsvertretungen wird beispielhaft deutlich, wenn Hersteller die Vertriebspolitik als zweitwichtigsten Bereich kooperativer Maßnahmen mit dem Handel in der Rezession einstufen (hinter der Produktpolitik); vgl. Ronlmann, P., Marketing in der Rezession. Ein Beitrag zur antizyklischen Produkt- und Programmolitik bei langlebigen Konsumgütern, Wiesbaden 1977, S. 159 f. und S. 204. Zur kritischen Situationsanalyse vgl. Voss, H., Wandel durch Nachdenken, Handelsvertreter-Praxis, Heft 11, Braunschweig 1979, S. 15 f:; Bless, H.J., Wieviel Freiraum hat der freie Handelsvertreter, in: VuM 1974, Heft 12, S. 3 ff.; Quitzau, H., Wer kann's besser, in: LZ Nr. 47 vom 24.11. 1978, S. 86. Eine umfassende Analyse quantitativer Entscheidungshilfen bei der Wahl von Absatzorganen belegen Dichtl,E., Raffée,M., Niedetzky,H.M., Reisende oder Handelsvertreter - Eine Anleitung zur Lösung eines Entscheidungsproblems mit praktischen Vorschlägen, Mannheim 1981.

2 Das Verständnis von Handelsvertretungen als Dienstleistungsunternehmen konkretisiert die rechtliche Begriffsbestimmung des $\S 84$ HGB auf seinen wirtschaftlichen Hintergrund ("Handelsvertreter ist, wer als selbständiger Gewerbetreibender ständig damit betraut ist, für einen anderen Unternehmer Geschäfte $z$ u vermitteln", $\$ 84$ Abs. 1, HGB); vgl. auch Bachmann, R., Das neue Recht des Handelsvertreters, Kissing 1978, S. $18 \mathrm{ff..} \mathrm{Die} \mathrm{Chancen} \mathrm{für} \mathrm{das}$ Marketing im Dienstleistungsbereich belegt Meffert, H., Die Perspektiven des Marketing in den 8oer Jahren, a.a.0., S. 31; besondere Profilierungsmöglichkeiten für Handelsvertretungen betont Tietz, B., Die Haupttendenzen für das Handelsmarketing in den 8oer Jahren, in:Marketing im Wandel, a.a.0.,S.117 f.; zur grundsätzlichen Einordnung der Handelsvertreteraktivitäten als Dienstleistungen vgl. Falk,B., Zur Bedeutung des Dienstleistungsmarketing, in:Dienstleistungsmarketing,Hrsg.:B.Falk, München 1980,S.16;Rathmell, J.M. , Marketing in the Service Sector, Cambridge Mass.1974, S.7; Meyer, P.W. , Tostmann, T. , Dienstleistungsmarketing, in: JAVF 1978 , S. 286 ff. insbes. S. 293. 
Als Ausgangspunkt einer Neuorientierung ist eine Standortbestimmung der Tätigkeit von Handelsvertretern Im Distributionssystem unverzichtbar. Eine differenzierte Kenntnis der Position von Handelsvertretungen im Absatzkanal bereitet den Weg zum Verständnis aktueller Entwicklungen bei Industrie und Handel und ihren Auswirkungen auf Handelsvertretungen. Die Formulierung von Konsequenzen bedarf dabei der Einbeziehung geeigneter Forschungsansätze, die eine strategische Orientierung des Marketing von Handelsvertretungen und eine projektive Maßnahmenplanung unterstützen.

2. Duale Stellung der Handelsvertretung im vertikalen Marketing

Eine Standortbestimmung der Handelsvertretung ${ }^{1}$ im system des Marketing deckt auf ${ }^{2}$, daß sich die Handelsvertretung als Absatzmittler zwischen einem Lieferanten- und Kundenmarkt bewegt, wobei beide Marktseiten aus Industrie- und Handelsbetrieben zusammengesetzt sein können. Abbildung 1 veranschaulicht den gesamten Tätigkeitsbereich von Handelsvertretungen ${ }^{3}$. Die größte Bedeutung kommt Handelsvertretungen dabei innerhalb der Produzentenstufe sowie zwischen Produktion und Groß- bzw. Einzelhandel zu (in der Abbildung 1 schraffiert) ${ }^{4}$.

1 Wegen der unternehmerischen Eigenständigkeit von Handelsvertretern wird für deren unternehmerische Belange der Begriff Handelsvertretung verwendet. Vom Handelsvertreter soll gesprochen werden, wenn unmittelbar auf die Person und ihre Eigenschaften abgezielt ist.

2 Zur Systembetrachtung des Marketing vgl. Meffert, H., Marketing. Einführung in die Absatzpolitik, 5. Aufl., Wiesbaden 1980, S. $39 \mathrm{ff.}$.

3 Vgl. Batzer, E., Meyerhöfer, W., Aufgaben, Stellung und Entwicklungsperspektiven der Handelsvertretung in der Bundesrepublik Deutschland, Berlin 1970, S. 26.

4 Vgl. ebenda,S.27; Meffert,H., Marketing,a.a.O., S.398. Es wird sogar die Ansicht vertreten, daß Handelsvertretungen "an allen entscheidenden Verbindungsstellen" des gesamten Wirtschaftsgefüges anzutreffen sind;vgl.Jeske,J.J.,a.a.O., S. 11 . 


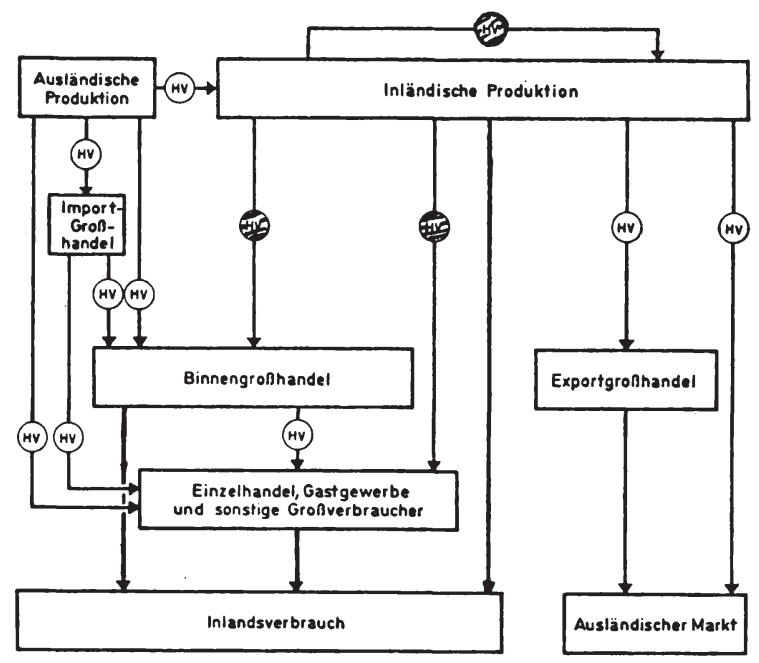

Abb. 1: Tätigkeitsbereiche von Handelsvertretungen

Die Abbildung dokumentiert die Vielschichtigkeit mehrstufiger Distributionssysteme und beleuchtet zugleich den Aufgabenbereich von Handelsvertretungen im vertikalen Marketing ${ }^{1}$.

1 Der Verkauf über Handelsvertreter wird auch als indirekter Absatz bezeichnet; vgl. Meffert, H., Marketing,a.a. O., S. 393 f.. Zur Begriffsbildung Distribution-Absatzkanal-vertikales Marketing vgl. insbes. die umfassende Ubersicht bei Maas, R.M., Absatzwege - Konzeptionen und Modelle, Wiesbaden 1980, S. 15 ff.; Ahlert, D., Grundzüge des Marketing, a.a.O., S. $198 \mathrm{ff.;}$ Steffenhagen, H., Vertikales Marketing, in: Marketing-Enzyklopädie, Band 2, München 1974, S. 675 ff.; Rosenbloom, B., Marketing Channels. A Management View, Hinsdale 1978, S. 48 f.; Stern, L.W., El-Ansary, A.I., Marketing Channels, Englewood Cliffs 1977, S. 56 ff.; Sims, J.T., Foster, J.R., Woodside, A.G., Marketing Channels - Systems and Strategies, New York usw. 1977, S. 6 f.. 
Die eingetragenen Positionen der Handelsvertreter in diesem System zeichnen sich gegenwärtig durch einen übereinstimmenden Kern ihres Tätigkeitsbereiches aus. Demnach"ist der Handelsvertreter ein Dienstleistungsunternehmer, der es übernimmt ... für einen anderen Unternehmer (z.B. eine Industriefirma oder einen Importeur) in einem bestimmten Bezirk die Aufgabe des Verkaufens im weitesten Sinne zu übernehmen" 1 .

Die Einordnung als Dienstleistungsunternehmen im Vertriebsbereich bringt gleichzeitig zum Ausdruck, daß Funktionen und Leistungen von Handelsvertretungen nur aus der dynamischen Entwicklung der Vertriebsformen im vertikalen Marketing erklärt werden können ${ }^{2}$.

Die Entwicklung bei den unmittelbaren Marktpartnern von Handelsvertretungen ist in den letzten Jahren durch tiefgreifende Wandlungen gekennzeichnet. Kooperation und Konzentration bei Industrie und Handel beleuchten schlaglichtartig diesen Proze ${ }^{3}$. Eingebettet in diese Entwicklung

1 Voss, H., Art.: Handelsvertreter, in: Management-Enzyklopädie, 3. Band, München 1970, S. 415 ff..

2 Vgl. Meffert, H., Die Handelsvertretung im Spannungsfeld des Marketing-Systems, a.a.0., s. $83 \mathrm{f}$..

$3 \mathrm{Vgl}$. beispielhaft Meffert, H., Perspektiven des Marketing in den 8oer Jahren, a.a.0., S. $21 \mathrm{f}$.; Steffenhagen, H., Konflikt und Kooperation in Absatzkanälen, Wiesbaden 1975, S. 21 f.; 0.V., Welche Auswirkungen haben Kooperation und Konzentration, in: Lz Nr. 41 vom 13.10.1978, S. 8; empirische Beispiele dieser Problematik belegen Meffert, H., Steffenhagen, H., Konflikte zwischen Industrie und Handel - Empirische Untersuchungen im Lebensmittelsektor der BRD, Wiesbaden 1976 sowie einige Beiträge in Meffert, H., (Hrsg.), Marketing heute und morgen. Entwicklungstendenzen des Marketings in Theorie und Praxis, Wiesbaden 1975, S. 299 ff.. 
können "ein wirkungsvolles Herstellermarketing einerseits und ein sich zunehmend profilierendes Händlermarketing andererseits ... auch für die Handelsvertretungen nicht ohne Einfluß bleiben" ${ }^{1}$. So sehen sich Handelsvertretungen beispielsweise verstärkt Forderungen beider Marktpartner gegenüber, die auf eine Ausweitung der bisherigen Leistungen abzielen. Derartige Funktions- und Risikoverlagerungen (z.B. Herstellerwerbung, Delkredere, Regalpflege) bieten dabei vielfach $A n l a B$ zu Meinungsverschiedenheiten und Konflikten ${ }^{2}$.

Unter dem Einfluß der beschriebenen situativen Veränderungen bei Industrie und Handel haben sich offensichtlich die Meinungen und Vorstellungen und insbesondere die Erwartungen an das Dienstleistungsangebot von Handelsvertretungen grundlegend gewandelt. Um dem ständigen Zwang zur Anpassung wirksam zu begegnen und um aktuelle Marktentwicklungen besser antizipieren zu können, ist es erforderlich, daß "die Handelsvertretungen auf die Veränderungen bewußt eingehen und ein eigenständiges und systematisches Handelsvertreter-Marketing betreiben" ${ }^{3}$. Die Besonderheit eines solchen Marketing begründet sich aus der dualen Einbindung einer Handelsvertretung in den Absatzkanal und der damit einhergehenden zweiseitigen Wirksamkeit ihrer absatzpolitischen Instrumente.

Den Gedanken, beide Marktpartner in das HandelsvertreterMarketing zu integrieren, veranschaulicht Abbildung 2.

1 Meffert, H., Die Handelsvertretung im Spannungsfeld des Marketing-Systems, a.a.O., S. 84 .

2 Vgl. ebenda, S. $84 \mathrm{f}$. sowie o.V., Konflikte erkennen und vermeiden lernen, in: Lz Nr. 9 vom 2.3.1979, S. 104.

3 Meffert, H., Die Handelsvertretung im Spannungsfeld des Marketing-Systems, a.a.O., S. 135; vgl. auch Koinecke, J., Marketing mit Handelsvertretern im härteren Markt, in: HV + HM 1976, S. 195. 


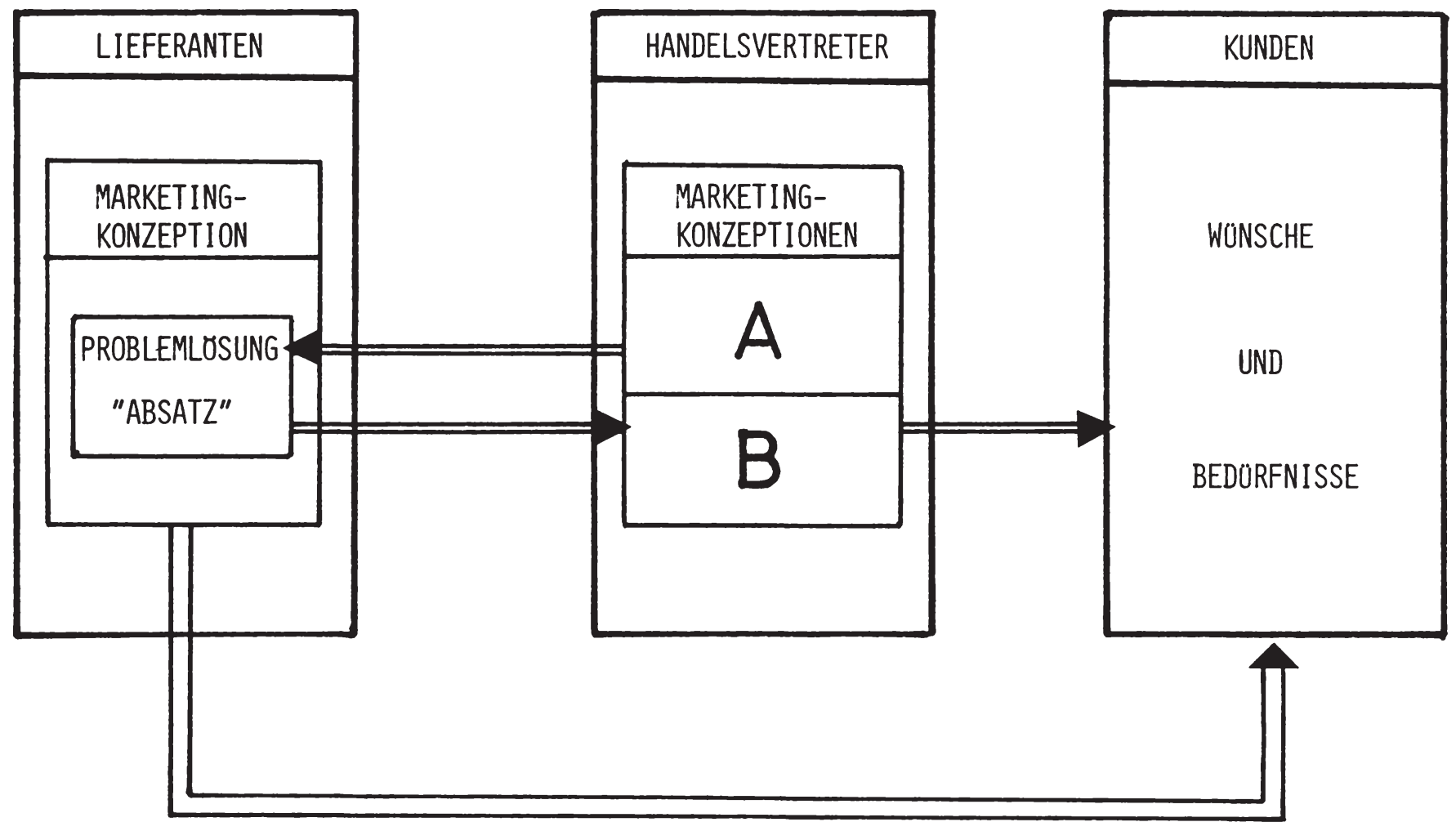

$\infty$

Abb. 2: Dualistische Marketingkonzeption von Handelsvertretungen 
Die Zweiseitigkeit dieser Orientierung kommt darin zum Ausdruck, daß gegenüber jeder 'Zielgruppe' eine spezifische Marketingkonzeption vorliegt (Konzeptionen $A$ und $B)^{1}$. Dabei ist weniger an zwei isolierte Dienstleistungsbündel gegenüber Kunden und Herstellern gedacht; vielmehr gilt es, eine einheitliche und sich ergänzende Konzeption aufzubauen. Zentrale Gestaltungsmerkmale dieses dualistischen Konzeptes sind:

"- Die Handelsvertretung soll "Vollstrecker" des Marketing der von ihr vertretenen Unternehmungen sein (Konzeption B).

- Die Handelsvertretung hat als Dienstleistungsunternehmen ein eigenes Marketing unter dem Gesichtspunkt des eigenen Geschäfts zu treiben (Konzeption A) 12 .

Die Marketingkonzeption vom Typ B stellt gleichsam den bisherigen - und damit weitgehend vorhandenen - Teil eines Handelsvertreter-Marketing dar. Eine wirksame Reaktion auf beschriebene Marktentwicklungen und Veränderungen im Vertriebsbereich erfordert jedoch von den Handelsvertretern eine Umgewichtung traditioneller Denkweisen. Profilierung gegenüber Mitbewerbern (d.h. anderen Handelsvertretungen und

1 Die Zweiseitigkeit eines Handelsvertreter-Marketing betont erstmals Meffert, H., Die Handelsvertretung in den 8oer Jahren, in: HV + HM 1977, S. 788.

2 Voss, M., Handelsvertreter-Marketing, in: Handbuch Marketing, Band 2, Hrsg.: J. Koinecke, Gernsbach 1978, S. 1434 (Ergänzungen vom Verfasser); die generelle Aufgabenstellung für Handelsvertretungen wurde treffend als 'creative catalyst' zwischen Industrie und Handel umschrieben, vgl. Newton, D.J., Role of Premium Rep more than a Salesman, in: Advertising Age, Nr. 42 vom 16. Oktober 1978, s. 18 f.. 
anderen Vertriebsformen) und damit langfristige Absicherung des eigenen Unternehmens sind letztlich nur bei einer ausgereiften Marketingkonzeption vom Typ A möglich. Diese zielt darauf $a b$, aktuellen und potentiellen vertragspartnern das Leistungsangebot der Handelsvertretung als geschlossenes Dienstleitungspaket transparent zu machen, "denn sie sind die eigentlichen Kunden der Handelsvertretung als Abnehmer ihrer Dienstleistung" ${ }^{1}$.

Die Konzipierung eines derartigen dualistisch orientierten Handelsvertreter-Marketing erfordert bei einer anzustrebenden Ausrichtung an den realen Gegebenheiten ${ }^{2}$ die unmittelbare Einbeziehung der beschriebenen Veränderungen in den Vorstellungen und Erwartungen der Marktpartner. Konkret bedeutet dies, daß ein Ausgleich möglicherweise divergierender Interessen bei der Gestaltung des Dienstleistungsprogramms nur herbeigeführt werden kann, wenn einer Handelsvertretung die an ihre Position geknüpften Erwartungen bekannt sind. Nur unter diesen Voraussetzungen ist eine Handelsvertretung in der Lage, ihre Rolle im Absatzkanal entsprechend auszufüllen ${ }^{3}$. Für die Konzipierung eines Handelsvertreter-Marketing und damit zur Gestaltung und Pflege der Beziehungen im Absatzkanal stellt demnach die

1 Voss, H., Handelsvertreter-Marketing, a.a.O., S. 1434.

$2 \mathrm{Zu}$ einer an "Echtzeitproblemen" orientierten MarketingWissenschaft vgl. Meffert, H., Perspektiven des Marketing in den 8oer Jahren, a.a.O., S. 32; Möglichkeiten des Transfers wissenschaftlicher Erkenntnisse auf praktische Problemstellungen analysiert Kroeber-Riel, W., Konsumentenverhalten, 2. Auf1., München 1980, S. $42 \mathrm{ff} .$.

3 Bis zu einer endgültigen - d.h. handelsvertreterspezifischen - Festlegung des Begriffs 'Rolle' wird als Arbeitsdefinition verwendet: Rolle = Verhaltenserwartungen an Mitglieder eines sozialen Systems; vgl. Steffenhagen, H., Konflikt und Kooperation in Absatzkanälen, a.a.O., S. 47 . 
Analyse der Rolle von Handelsvertretungen einen zentralen Ansatzpunkt dar. Eine Analyse von Rollen und Rollenbeziehungen im vertikalen Marketing spiegelt damit zugleich die dualistische Struktur eines Handelsvertreter-Marketing wider.

Die grundsätzliche Eignung rollentheoretischer Uberlegungen zur Analyse der Beziehungen in Distributionssystemen wurde im Zusammenhang mit einer funktionalen Betrachtung einzelner Marktpartner bereits früh erkannt:
"Zwar ist die Funktionenlehre ... letztlich eine Lehre von der Rollendifferenzierung im Marketing-System. Umso mehr erstaunt es, wie wenig sie sich mit dem Rollenkonsens und -dissens beschäftigt. Relativ sta- bile Rollenstrukturen und ein diesbezüglicher Konsens zwischen den Systemteilnehmern bedeuten, daß letztere das Marktverhalten wechselseitig recht sicher prognostizieren können. Rollendissens ist dagegen eine weit verbreitete, typische Ursache für Konflikte" ${ }^{1}$.

Eine Umsetzung dieser Erkenntnisse in eine theoriegeleitete empirische Absicherung fand jedoch nur peripher statt ${ }^{2}$.

1 Meffert, H., Die Leistungsfähigkeit der entscheidungsund systemorientierten Marketing-Theorie, in: Wissenschaftsprogramm und Ausbildungsziele der Betriebswirtschaftslehre, Hrsg.: G.v. Kortzfleisch, Berlin 1971, S. 172. Neben den hier genannten Rollenbeziehungen werden ziel-, Macht- und Kommunikationsbeziehungen im vertikalen Marketing diskutiert. Vgl. etwa Steffenhagen, H., Konflikt und Kooperation in Absatzkanälen, a.a.O., s. $45 \mathrm{ff} . ;$ Meffert, H., Verhaltenswissenschaftliche Aspekte vertraglicher Vertriebssysteme, in: Vertragliche Vertriebssysteme zwischen Industrie und Handel, Hrsg.: D. Ahlert, (Wiesbaden 1981 - im Druck), S. 110 ff.; Stern, L.W., Brown, J.W., Distribution Channels: A Social Systems Approach, in: Distribution Channels: Behavioral Dimensions, Hrsg.: L.W. Stern, Boston 1969, S. 17. Ausgehend von der Rollenanalyse als zentralem Untersuchungsbereich werden die genannten Beziehungen im Einzelfall zur Interpretation aufgedeckter Rollenbeziehungen ergänzend herangezogen.

2 Vgl. Steffenhagen, H., Konflikt und Kooperation in Absatzkanälen, a.a.o., s. 91. 
Gleichwohl ermutigen bisherige Forschungsansätze den Versuch, eine Rollenanalyse auf empirischer Basis als Grundlage des dualistischen Handelsvertreter-Marketing durchzuführen. "Nevertheless, many of the concepts and propositions of role theory provide a promising means of analyzing channels of distributions as social systems" ${ }^{1}$.

\section{Ziel und Gang der Untersuchung}

Ausgehend von dem Grundgedanken der dualen Einbindung in das vertikale Marketing liegt das $\underline{\text { ziel }}$ der vorliegenden Untersuchung in einer rollenorientierten Analyse der Dienstleistungen bzw. des Marketing von Handelsvertretungen. Diese rollenbezogene Vorgehensweise verfolgt dabei zwei Aspekte. Zum einen gilt es zu prüfen, ob sich die bisher vorliegenden theoretischen Ansätze einer Rollenanalyse in Distributionssystemen auf die Problembereiche des Marketing von Handelsvertretungen transferieren lassen. Der zweite Aspekt zielt auf eine operationale Umsetzung rollentheoretischer Uberlegungen im Handelsvertreter-Marketing im Hinblick auf eine umfassende empirische Analyse der Rolle von Handelsvertretungen. Die vorliegende Arbeit bezieht sich damit sowohl auf theoretische als auch auf empirische Fragestellungen. Der bisher deutlich werdende explikative Charakter der Arbeit steht dabei in unmittelbarer Verbindung mit praktisch-normativen Aussagen über Maßnahmenplanungen im Handelsvertreter-Marketing. Insofern kommt der empirischen Untersuchung und den daraus ableitbaren Konsequenzen eine besondere Bedeutung $\mathrm{zu}$.

1 Gill, L.E., Stern, L.W., Roles and Role Theory in Distribution Channel Systems, in: Distribution Channels: Behavioral Dimensions, a.a.O., S. 23. 
Ziele und Schwerpunkte der Arbeit sind demnach zusammenfassend:

1. Entwicklung eines verhaltenswissenschaftlichen Bezugsrahmens zur Rolle von Handelsvertretungen im vertikalen Marketing

2. Umsetzung dieser theoretischen Konzeption in eine umfassende empirische Untersuchung.

Diese generellen Ziele lassen sich durch differenzierte programmfragen präzisieren:

(1) Eignet sich das Rollenkonzept zur Erfassung der dualen Einbindung von Handelsvertretungen im Absatzkanal?

(2) Welches sind die zentralen Rollen von Handelsvertretungen?

(3) Bestehen Zusammenhänge zwischen der Rolle von Handelsvertretungen und allgemeinen Merkmalen der Marktpartner, die sich für die Gestaltung von Marketingmaßnahmen nutzen lassen?

(4) Welche Konsequenzen ergeben sich aus der Rollenanalyse für das dualistische HandelsvertreterMarketing?

Entsprechend der beschriebenen Zielsetzungen ist der Gang der Untersuchung konzipiert. Im Kapitel B wird zunächst der verhaltenswissenschaftliche Bezugsrahmen einer Rollenanalyse im Handelsvertreter-Marketing aufgebaut. Dieser Ansatz mündet in ein theoriegeleitetes Modell, das mit 
seinem Hypothesengerüst den Ausgangspunkt der empirischen Untersuchungen bildet.

Der empirische Teil der Arbeit (Kapitel C) orientiert sich an den beschriebenen Programmfragen der Untersuchung, die in Form expliziter Hypothesen verfeinert werden. Nach einem konzeptionellen Teil zur Beschreibung der empirischen Studie werden in vier Schwerpunkten die grundsätzliche Eignung des Rollenkonzeptes, differenzierte Rollenausprägungen und -beziehungen sowie Ansätze einer rollenbezogenen Segmentierung der Marktpartner im Handelsvertreter-Marketing überprüft. Die Auswirkungen der empirischen Befunde für Handelsvertretungen sowie Anstöße weiterer Forschungen faßt abschließend Kapitel D zusammen. 


\section{B. Verhaltenswissenschaftlicher Bezugsrahmen eines Han- delsvertreter-Marketing}

Ausgangspunkt für die Ermittlung eines dienstleistungsorientierten Marketing von Handelsvertretungen muß eine eingehende Analyse der aktuellen Stellung der Handelsvertretung im vertikalen Marketing sein. Diese Frage nach der gegenwärtigen Rolle von Handelsvertretungen erfordert eine Präzisierung im Hinblick auf die dualistische Einbindung einer Handelsvertretung in den Absatzkanal. Es gilt daher, zunächst das Konstrukt 'Rolle' in seiner handelsvertreterspezifischen dualen Ausrichtung zu explizieren. Eine differenzierte Analyse des Rollenbegriffs soll daher den Weg $\mathrm{zu}$ einer umfassenden Diskussion möglicher Rollenkomponenten sowie -beziehungen im dualistischen HandelsvertreterMarketing öffnen. Die empirische Fundierung eines Handelsvertreter-Marketing macht es darüber hinaus erforderlich, solche Faktoren in die Analyse einzubeziehen, die die Ausprägungen der Rolle von Handelsvertretungen beeinflussen (z.B. Vertriebsstrukturen, Branchen, Unternehmensgrößen). Erst die Gesamtheit dieses Bezugsrahmens vermag den für ein Handelsvertreter-Marketing strategisch bedeutsamen Erklärungszusammenhang aufzudecken.

1. Rollenanalyse als Grundlage eines dualistischen Handelsvertreter-Marketing

Die Analyse der Rolle der Handelsvertretung erfordert zunächst eine begriffliche Klärung des Konstrukts 'Rolle', Dabei gilt es zu überprüfen, ob sich dieser erst in der jüngeren Diskussion im vertikalen Marketing verwendete Terminus auf den Bereich des Handelsvertreter-Marketing übertragen läßt und welche spezifischen Besonderheiten sich aus der dualen Stellung der Handelsvertretung für eine Rollenanalyse ergeben. 


\subsection{Begriffliche Grundlagen der Rollenanalyse}

Der Rollenbegriff hat in der verhaltenswissenschaftlichen Forschung vielfach Verwendung gefunden, wobei eine Vielzahl unterschiedlicher Bedeutungsinhalte anzutreffen ist. Dabei hat sich keine allgemeingültige Begriffsklärung herausgebildet. Es gilt daher, die Ansätze verschiedener Forschungsrichtungen im Hinblick auf eine Verwendung im Rahmen des Handelsvertreter-Marketing $z u$ vergleichen.

Der Rollenbegriff geht auf kulturanthropologische Forschungen zurück. Einstellungen, Wert vorstellungen und Verhaltensweisen, die dem Inhaber eines sozialen Status von der Gesellschaft zugeschrieben werden, bilden die zentralen Merkmale des Begriffs ${ }^{1}$. Restriktiv für die vorliegende Problemstellung wirkt sich aus, daß dieser Forschungsansatz zwischen den realisierten Rollenerfüllungen und

$1 \mathrm{Vgl}$. Linton, R., The Study of Man, New York 1936; ders., The Cultural Background of Personality, London 1945. Für die betriebswirtschaftlich-ökonomisch ausgerichtete Forschung sind insbesondere die Relativität sozialer Wertsysteme sowie angesichts wechselnder Internationalisierung der Märkte die ermittelte interkulturelle Beliebigkeit von Rolleninhalten hervorzuheben. Zur Bedeutung individueller Wertsysteme im Marketing vgl. Dahlhoff, H.D., Individuelle Wertorientierungen Analyse und Aussagewert personenspezifischer Werthierarchien im Marketing, Arbeitspapiere des Instituts für Marketing der Universität Münster, Hrsg. H. Meffert, Nr. 23, Münster 1980, S. 4 f.. Beispiele für einen unterschiedlichen interkulturellen Bedeutungsgehalt verwendeter Begriffe belegt Althans, J., Standardisierung von Marketingkonzeptionen in internationalen Unternehmen aus der Sicht von Tochtergesellschaften - Eine explorative Studie auf einem überseeischen Markt, Arbeitspapiere des Instituts für Marketing der Universität Münster, Hrsg.: H. Meffert, Nr. 20, Münster 1980, S. 20. 
möglichen Rollenerwartungen nicht differenziert und sich damit eines wesentlichen Aspektes empirischer Forschung verschließt ${ }^{1}$.

Eine stärkere Explizierung hat der Rollenbegriff in der soziologischen Forschung gefunden ${ }^{2}$. Ohne im Detail die Einzelheiten der hier vorherrschenden klassifikatorischtaxonomischen Bemühungen aufzugreifen, lassen sich zwei zentrale Rollenkonzepte ausmachen ${ }^{3}$. Nach dem normativen Konzept sind Rollen eine Reihe von Erwartungen ${ }^{4}$, die gegenüber dem Inhaber einer sozialen Position bestehen ${ }^{5}$.

1 Vgl. Wiswede, G., Rollentheorie, Stuttgart 1977, S. 14 sowie Sader, M., Rollentheorie, in: Handbuch der Psychologie, 7. Bd., Sozialpsychologie, 1. Halbband, Göttingen 1975.

$2 \mathrm{Zu}$ einem umfassenden Uberblick vgl. Popitz, M., Der Begriff der sozialen Rolle als Element der soziologischen Theorie, Tübingen 1967.

3 Zur Diskussion des Rollenbegriffs in der Soziologie vgl. Dahrendorf, R., Homo Sociologicus, 13. Aufl., Opladen 1974, S. 25 ff.; ders., Rolle und Rollentheorie, in: Wörterbuch der Soziologie, Hrsg.: W. Bernsdorf, Stuttgart 1969, S. 902 ff.; Sarbin, T.R., Turner, R.H., Role, in: International Encyclopedia of the Social Sciences, Hrsg.: D.L. Sills, Vol. 13, New York 1972, S. $546 \mathrm{ff}$.; Claessens, D., Rolle und Macht, 2. Aufl., München 1970, S. $11 \mathrm{ff}$. sowie Tenbruck, F.M., Zur deutschen Rezeption der Rollentheorie, in: KzfSS 1961, S. 1 ff.; Levinson, D.J., Role, Personality and Social Structure in the Organizational Setting, in: Sociology, Hrsg.: S.M. Li pset, N.J. Smelser, Englewood Cliffs 1961, S. 299 ff.. Einen Gesamtüberblick rollentheoretischer Abhandlungen geben Biddle und Thomas, vgl. Biddle, B.J., Thomas, E.J. (Hrsg.), Role Theory, Concepts and Research, New York usw. 1966, S. $8 \mathrm{f}$. und S. 16 .

4 Zur Entstehung von Erwartungen aufgrund von Interaktionen in einem sozialen system vgl. Bales, R.F., Task roles and social roles in problem solving groups, in: Current studies in social psychology, Hrsg.: I.D. Steiner, M. Fishbein, New York 1965, S. $321 \mathrm{ff.;}$ Sherif, C.W., Orientation in Social Psychology, New York usw. 1976, S. 60 f..

5 Zur Differenzierung soziologischer Rollenkonzepte vgl. Wiswede, G., a.a.O., S. 15; Banton, M., Roles - An Introduction to the Study of Social Relations, London 1965, S. 29 ff.; Sarbin, T.R., Allen, V.L., Role Theory, in: The Handbook of Social Psychology, Hrsg.: G. Lindzey, E. Aronson, Reading 1968, S. 488 ff.; zum normativen Rollenbegriff vgl. Thomas, E.J., Biddle, B.J., Basic Concepts for Classifying the Phenomena of Role, in: Role Theory: Concepts and Research, a.a.o., S. $23 \mathrm{ff}$. sowie die dort ansegebene Literatur. 
Gegenüber der ursprünglichen Definition von Linton wird hier der Begriff 'Status' durch den der 'Position' ersetzt, um auf diese Weise den eher im Sinne von Prestige verwendeten Statusbegriff durch einen mehr auf konkrete Funktionen ausgerichteten Begriff $z u$ versachlichen ${ }^{1}$. Hinsichtlich der Stellung einer Handelsvertretung im Absatzkanal erweist sich diese Modifikation als vorteilhaft, läßt sich doch die Position einer Handelsvertretung - z.B. durch ein bestimmtes Aufgabenprofil - relativ klar abgrenzen. Weniger geeignet ist das normative Rollenkonzept dagegen zu beurteilen, wenn es um eine Weiterentwicklung des gegenwärtigen Leistungsangebotes von Handelsvertretungen in ein an den zukünftigen Erwartungen orientierten Dienstleistungsprogrammsgeht. Hier erweist sich die alleinige Fixierung auf die Erwartungshaltungen an Handelsvertretungen als $\mathrm{zu}$ einseitig. Insbesondere die fehlende Relativierung im Vergleich zum bestehenden Dienstleistungsangebot steht der strategischen Ausrichtung eines Handelsvertreter-Marketing entgegen.

Ein weiter gefaßter Rollenbegriff liegt dem behavioralen Rollenkonzept zugrunde. Hier versteht man unter Rolle ein Verhalten, das gegenüber den Inhabern anderer sozialer Positionen ausgeübt wird ${ }^{2}$. Im Vergleich zum normativen

1 Vgl. Claessens, D., a.a.O., S. 19; Thomas, E.J., Biddle, B.J., Basic Concepts for Classifying the Phenomena of Role, a.a.O., S. 28 f.. Daneben werden die Begriffe Status und Position teilweise synonym verwendet, vgl. Gill, L.E., Stern, L.W., a.a.O., S. 33; Kirsch, W., Entscheidungsprozesse, 3. Band, Wiesbaden 1971, S. 101. Zur grundlegenden Abgrenzung des Positionsbegriffs vgl. Gross, N. , Mason, S.W. , McEachern, W.A., Explorations in Role Analysis, New York 1958, S. $48 \mathrm{ff} .$.

2 Vgl. Wiswede, G., a.a.O., S. 15; Merton, R.K., Social Theory and Social Structure, Glencoe 1957, S. $116 \mathrm{ff}$. und S. $336 \mathrm{ff}$. 
Rollenbegriff werden die an einen Positionsinhaber gerichteten Erwartungen nicht explizit als Bestandteil seiner Rolle verstanden, sie finden indirekt ihren Ausdruck in dem realisierten Verhalten. "Die Diskussion der genannten kontroversen Standpunkte zu Inhalt und Funktion des Rollenbegriffs scheint demnach kaum eine Begriffsbestimmung zuzulassen, die nicht in irgendeiner Weise als unangemessen oder restriktiv angesehen wird" ${ }^{1}$.

Den Problemen um die theoretischen Dimensionen des Rollenbegriffs versucht die sozialpsychologische Forschung durch eine Ausrichtung auf operational brauchbare Definitionen $\mathrm{zu}$ begegnen. Insbesondere organisationale Forschungsansätze verwenden einen konkreteren Rollenbegriff ${ }^{2}$. So wird etwa die Rolle verstanden als Bündel unabhängiger Aktivitäten, die in ihrer Kombination das Verhalten eines Positionsinhabers $^{3}$ oder - auf einer höheren Aggregationsebene das Verhalten von Organisationen ausmachen ${ }^{4}$.

Der Positionsbegriff findet dabei in einer ausgeweiteten Fassung Anwendung. Demnach umfaßt eine Position "eine

1 Wiswede, G., a.a.0., s. 16 .

2 Vgl. ebenda, S. 21 sowie Rosenstiel, L.v., Grundlagen der Organisationspsychologie, Stuttgart 1980, S. 47 f..

$3 \mathrm{Vgl}$. Herbert, T.T., Dimensions of Organizational Behavior, New York-London 1976, S. 316; Luhmann, N., Funktionen und Folgen formaler Organisation, Berlin 1964, S. 34 f.; Hayntz, R., Soziologie der Organisation, Reinbek 1963, S. 81 ff..

4 Vgl. Katz, D., Kahn, R.L., The Social Psychology of Organizations, New York 1966, S. 179; Parsons, T., Shils, E.A., The Content of Roles, in: Role Theory, Concepts and Research, a.a.O., S. 239. 
Menge von Personen, die gleiche Merkmale aufweisen ... oder für die ein Bündel einzigartiger Kognitionen entweder von ihnen selbst oder von anderen gehalten wird" ${ }^{1}$. Diese mit einer Position assoziierten Informationen betreffen zum einen das äußere Verhalten bzw. sichtbare Eigenschaften eines Positionsinhabers (faktische Informationen).Daneben sind zugleich mutmaßliche Einstellungen, Uberzeugungen und Werte Bestandteil dieser kognitiven Informationen (wertende Informationen) ${ }^{2}$. Eine Rolle ist demzufolge "der Inbegriff der kognitiven Informationen faktischer und wertender Art, die im Individuum mit einer Position assoziiert" ${ }^{3}$.

Dieser Rollenbegriff umfaßt in seiner Ausrichtung auf faktische und wertende Bestandteile die zuvor diskutierten Konzepte des normativen bzw. behavioralen Rollenverständnisses. Gleichzeitig schließt diese Definition"nicht aus, daß es der Positionsinhaber selbst ist, der über die die Rolle konstituierenden Informationen bezüglich der Position verfügt. Die entsprechenden kognitiven Informationen des Positionsinhabers umfassen sowohl sein eigenes 'Wissen' über diese Position als auch sein Wissen von den Erwartungen und Normen, die andere mit dieser Position verbinden" 4 . Das Zusammenwirken der diskutierten rollen-

1 Biddle, B.J., The Present Status of Role Theory, Columbia 1961, S. 5 in der Ubersetzung von Kirsch, W., Entscheidungsprozesse, 3. Band, a.a.0., s. 101.

2 Vgl. Kirsch, W., Entscheidungsprozesse, 3. Band, a.a.0., S. 102. Die von Kirsch diskutierten Komponenten kognitiver Informationen über eine Position werden auch als generelle verhaltensspezifische Rollen diskutiert (Overt role, Covert role, Prescriptive role, Descriptive role, Evaluative role, Active role, Sanctioning role), vgl. Thomas, E.J., Biddle, B.J., Basic Concepts for Classifying the Phenomena of Role, a.a.0., s. 31.

3 Kirsch, W., Entscheidungsprozesse, 3. Band, a.a.0.,s. 102 .

4 Ebenda, S. 102 f.; die besondere Bedeutung des Selbstverständnisses des Positionsinhabers für die Rollenausübung wird betont bei Porter, L.W., Lawler, E.E., Managerial Attitudes and Performance, Homewood 1968, S. 24 f.; Behling, O., Schriesheim, C., Organizational Behavior, Boston 1976, S. 84 . 
bezogenen Merkmale in einem sozialen system ${ }^{1}$ veranschaulicht Abbildung $3^{2}$.

\begin{tabular}{|c|c|c|c|}
\hline \multicolumn{2}{|c|}{ ROLE SENDERS } & \multicolumn{2}{|c|}{ FOCAL PERSON } \\
\hline ROLE & SENT & RECEIVED & ROLE \\
\hline EXPECTATIONS & ROLE & ROLE & BEHAVIOR \\
\hline
\end{tabular}

Abb. 3: Grundmodell der allgemeinen Rollenanalyse

1 Zum allgemeinen Zusammenhang zwischen Rollen und ihren umgebenden sozialen systemen vgl. Parsons, T., The Social System, Glencoe 1964, insbes. S. 25; eine Integration verhaltenswissenschaftlicher Rollenanalysen mit systemorientierten Strukturanalysen dokumentiert Meffert, H., Absatztheorie, systemorientierte, in: HdA, Hrsg.: B. Tietz, Stuttgart 1974, Sp. $138 \mathrm{ff}$. und insbesondere Meffert, H., Die Leistungsfähigkeit der entscheidungs - und systemorientierten Marketing-Theorie, in: Wissenschaftsprogramm und Ausbildungsziele der Betriebswirtschaftslehre, Tagungsberichte des Verbandes der Hochschullehrer für Betriebswirtschaft e.V., Hrsg.: G.v. Kortzfleisch, Berlin 1971, S. 172 .

2 Das beschriebene Rollenmuster wird auch als 'role episode' diskutiert, vgl. Katz, D., Kahn, R.L., a.a.O., S. 182 ff.; Herbert, T.T., a.a.O., S. 317 ff.; Kahn, R.L., u.a., Organizational Stress: Studies in Role Conflict and Ambiguity, New York usw. 1964, S. 26 ff.. 
Die Rollenerwartungen der Mitglieder (role senders) eines abgegrenzten sozialen Systems - im vorliegenden Fall des Absatzkanals - sind Ausdruck bewerteter Vorstellungen (role expectations) in Bezug auf das Verhalten der Mitglieder, die im Mittelpunkt des Interesses stehen (focal persons). In der übermittelten Rolle (sent role) erreichen diese Erwartungen über festgeschriebene (z.B. vertragliche) oder informale Beziehungen den Empfänger und bilden damit seinen kognitiven Informationsstock ${ }^{1}$. Die externen Rollenerwartungen formen beim Empfänger zusammen mit seinen eigenen Vorstellungen über die Art und Weise der Rollenausübung seine empfangene Rolle (received role) und münden in ein tatsächliches Rollenverhalten ${ }^{2}$.

Ubbertragen auf die Stellung der Handelsvertretung bedeutet dies, daß die Marktpartner im Absatzkanal Erwartungen an die Rolle einer Handelsvertretung stellen. Die bis-

1 Der Begriff Informationsstock lehnt sich an einen in der Käuferverhaltensforschung verwendeten Terminus an, der eine relativ stabile Informationsbasis - z.B. aufgrund wiederholten Informationszuflusses - bezeichnet. Vgl. hierzu beispielhaft Belonax, J.A., Mittelstaedt, R.A., Evoked set size as a function of number of choice criteria and information variability, in: Advances in Consumer Research, Hrsg.: H.K. Hunt, Provo 1978, S. $48 \mathrm{ff} .$, sowie den Uberblick bei Meffert, H., Steffenhagen, H., Freter, H., (Hrsg.), Konsumentenverhalten und Information, Wiesbaden 1979, S. 27 f.; Hughes, D.G., Ray, M.L. (Hrsg.), Buyer/Consumer Information Processing, Chapel Hill 1974.

2 Die beschriebene Grundstruktur rollentheoretischer tberlegungen hat in jüngerer zeit Eingang in das vertikale Marketing gefunden. Insgesamt liegen jedoch erst wenige Arbeiten vor, die die Eignung der Rollenanalyse für absatzwirtschaftliche Fragestellungen herausarbeiten, vgl. hierzu insbesondere G1ll, L.E., Stern, L.W., Roles and Role Theory in Distribution Channel Systems, a.a.O., S. 23 und S. 36 ff.; Steffenhagen, H., Konflikt und Kooperation in Absatzkanälen, a.a.O., s. 47 und $85 \mathrm{ff.}$. 
herige Wahrnehmung dieser Erwartungen in Verbindung mit dem Selbstverstänanis von Handelsvertretern über ihre eigene Position hat $\mathrm{zu}$ einer konkreten Rollenausübung geführt. Anders ausgedrückt: Die Position der Handelsvertretung im Absatzkanal führt zu einer Ubernahme spezifischer Marketingfunktionen. Diese Ubernahme ist Ausdruck rollendeterminierten Verhaltens und resultiert aus der zweiseitigen Orientierung einer Handelsvertretung im Absatzkanal. Besonders hervorzuheben ist, daß die Ubernahme von Funktionen dabei nicht aus der Position selbst, sondern allein aus dem rollendeterminierten Verhalten resultiert, d.h. "die Durchführung bzw. das Unterlassen spezifischer Transformationen durch den Positionsinhaber ... sind demnach als typische Rollen in Distributionssystemen zu interpretieren" $^{1}$.

Zusammenfassend hat sich damit die Eignung des Konstrukts 'Rolle' erwiesen, die Einbindung der Handelsvertretung in das vertikale Marketing und insbesondere die dualistische Grundstruktur eines Handelsvertreter-Marketing hinreichend zu beschreiben. "Der ursprünglich personenbezogene soziologische Rollenansatz wird also sozusagen als heuristischer Hebel benutzt, um institutionelle Phänomene zu klären" 2 .

1 Steffenhagen, H., Konflikt und Kooperation in Absatzkanälen, a.a.0., S. 86; vgl. auch Stern, L.W., Heskett, J.L., Conflict Management in Interorganization Relations, in: Distribution Channels: Behavioral Dimensions, a.a.0., S. 294 .

2 Krüger, W., Zur methodischen und inhaltlichen Problematik der Analyse von Zielbildungsprozessen, in: Unternehmensbezogene Konfliktforschung, Hrsg.: G. Dlugos, Stuttgart 1979, S. 388; die besondere Eignung der Rollentheorie als verbindendes Element zwischen den verschiedenen Ebenen eines sozialen Systems (Individuum - Gruppe - System) wird betont bei Grochla, E., Welge, M.K., Einführung in die verhaltenstheoretisch orientierten Ansätze, in: Organisationstheorie, Hrsg.: E. Grochla, 1. Teilband, Stuttgart 1975, S. 93. 
Für die weitere Rollenanalyse von Handelsvertretungen insbesondere deren empirische Ausrichtung - hat die bisherige Diskussion die Notwendigkeit deutlich werden lassen, die beschriebenen Teilaspekte des allgemeinen Rollenmodells einer differenzierten Analyse zu unterziehen. Dies gilt umso mehr, als das dualistische Handelsvertreter-Marketing - speziell im Hinblick auf die Ableitung maßnahmenorientierter Implikationen - eine Trennung der Rollenkomponenten auf Seiten der Marktpartner unabdingbar macht.

\subsection{Rollenkomponenten im Handelsvertreter-Marketing}

Auf der Basis des allgemeinen Rollenmodells (vgl. Abbildung 3) sind nach Maßgabe der begrifflichen Festlegung des Konstruktes 'Rolle' im folgenden die handelsvertreterspezifischen Ausprägungen einer Rollenanalyse aufzudecken. Die bisherige Diskussion zeigte an, daB sich das besondere Interesse dabei auf eine differenzierte Analyse rollenbezogener Merkmale bei allen einbezogenen Marktpartnern konzentrieren muß.

\subsection{Rollenausübung von Handelsvertretungen}

Betrachtet man zunächst die Handelsvertretung als Inhaber der zentralen Position im dualistischen Modell, so belegte diese allgemeine Rollenanalyse mehrfach die Bedeutung des Selbstverständnisses des Positionsinhabers für die Art und Weise, in der dieser seine Rolle ausfüllt ${ }^{1}$. Das Vorstellungsbild eines Handelsvertreters über seinen Tätigkeitsbereich formt somit einen wesentlichen Teil seiner Rolle im Absatzkanal. Zugleich fließen in diese

1 Vgl. Porter, L.W., Lawler, E.E., a.a.O., S. 24. 
Rolle die Informationen kognitiver Art über mögliche Erwartungen von seiten der Marktpartner ein ${ }^{1}$. So kann z.B. die Betreuung der Kunden über die reine Auftragserlangung hinaus zum Selbstverständnis eines Handelsvertreters zählen. Gleichzeitig weiß bzw. nimmt er an, daß die vertretenen Unternehmen z.B. die Bearbeitung von Reklamationen als Aufgabenbereich der Handelsvertretung ansehen. $\mathrm{Zu}$ ihrer Verknüpfung führen Selbstverständnis und kognitive Informationen über mögliche Rollenerwartungen $z u$ einer bestimmten Ausprägung des Verhaltens hinsichtlich der Reklamationsbearbeitung. Dieses realisierte Verhalten von Handelsvertretungen läßt sich als Rollenausübung bezeichnen. Damit sind Art und Umfang der von einer Handelsvertretung angebotenen Dienstleistungen als Ausdruck ihrer Rollenausübungen $\mathrm{zu}$ verstehen ${ }^{2}$.

Im Hinblick auf ein empirisch fundiertes Handelsvertreter-Marketing ist die Frage zu prüfen, in welcher Weise die Rollenausübung dabei Berücksichtigung finden mu $B$.

1 Kirsch, W., Entscheidungsprozesse, 3. Band, a.a.0., S. 102; zur Entstehung von Rollen vgl. ferner Thornton, R., Nardi, P.M., The Dynamics of Role Aquisition, in: AJS 1975, S. 870 ff. (abgedruckt in: Readings in Consumer Behavior: Individuals, Groups and Organizations, Hrsg.: Wallendorf, M., Zaltman, G. , New York usw. 1979, S. 126 ff.; Turner, R.M., Role-taking, role standpoint and reference-group behavior, in: AJS 1956, S. $316 \mathrm{ff}$. (wieder abgedruckt in: Behavioral Science Foundations of Consumer Behavior, Hrsg.: J.B. Cohen, New York usw. 1972, S. 389 ff..

2 Diese Festlegung erfolgt in Anlehnung an den in der Literatur anzutreffenden Terminus "RollenbewuBtsein". Dem Begriff Rollenausübung wird wegen seines eher funktions- bzw. aufgabenbezogenen semantischen Gehaltes hier der Vorzug gegeben; vgl. z.B. Steffenhagen, H., a.a.o., s. 86 und die dort angegebene Literatur. 
Greift man das obige Beispiel auf, so läßt sich der Dienstleistung 'Reklamationsbearbeitung' nicht unmittelbar ablesen, ob ein dominierendes Selbstverständnis in Bezug auf dieses Merkmal vorliegt, oder ob die kognitiven Informationen über die Erwartungen der Marktpartner zur tatsächlichen Ausübung dieser Leistung geführt haben. Die interne kognitive Gewichtung und Verknüpfung dieser Komponenten ist dem Ergebnis 'Rollenausübung' nicht mehr zu entnehmen ${ }^{1}$.

Stellt man den Aspekt der Planung von Maßnahmen im Handelsvertreter-Marketing in den Vordergrund, so wird offenkundig, daß die internen Prozesse, die zu einer Rollenausübung führen bzw. geführt haben, zur Lösung der hierbei auftretenden Probleme ${ }^{2}$ keinen unmittelbaren Beitrag leisten. Die Entwicklung von Dienstleistungen im dualen Handelsvertreter-Marketing muß vielmehr das Resultat derar-

1 Die beschriebene Problematik wird als Intrarollenkonflikt in der Literatur diskutiert, vgl. hierzu insbes. Wiswede, G., a.a.O., s. 115 ff.; Katz, D., Kahn, R.L., a. a.O., S. 184 ff.; Gross, N., McEachern, A.W., Mason, W.S., Role Conflict and its Resolution, in: Role Theory: Concepts and Research, a.a.0., S. $287 \mathrm{ff.;}$ zur grundlegenden Diskussion intraindividueller Konflikte vgl. Kirsch, W., Entscheidungsprozesse, 1. Band, Wiesbaden 1970, S. 96 ff. sowie Kamitz, R., Wissenschaftslogische Probleme beim Aufbau einer Theorie der Konflikte, in: Unternehmensbezogene Konfliktforschung, a.a.O., S. 33 ff..

2 Hier sei beispielhaft die Frage genannt, ob Bonitätsprüfungen Gegenstand der Leistungen von Handelsvertretungen sein sollten. Diese Frage läßt sich nur unter Einbeziehung des Verhältnisses Handelsvertretung-Hersteller beantworten. $\mathrm{Zu}$ den dabei auftretenden Divergenzen vgl. B. 1.3 dieser Arbeit. 
tiger kognitiver Prozesse als Ausgangspunkt wählen. Der status-quo gegenwärtiger Dienstleistungen, d.h. die tatsächliche Rollenausübung von Handelsvertretungen bildet damit den ersten Eckpfeiler einer Rollenanalyse im Handelsvertreter-Marketing.

\subsection{Rollenerwartungen an Handelsvertretungen}

Die duale Ausrichtung eines Handelsvertreter-Marketing macht die Einbeziehung der Vorstellungen und Meinungen der Marktpartner einer Handelsvertretung unverzichtbar. Insbesondere die Diskussion um die Komponenten der Rollenausübung von Handelsvertretungen hat dabei belegt, daB die Erwartungen der Marktpartner eine wesentliche Bedeutung für die Rollenanalyse des gesamten vertikalen Vertriebssystems aufweisen.

Die Notwendigkeit einer Einbeziehung von Erwartungshaltungen wird angesichts der damit auftretenden prognostischen Relevanz noch deutlicher. Diese prognostische Relevanz leitet sich unmittelbar ab aus einer Gegenüberstellung von Rollenausübung der Handelsvertretung und Erwartungen der Marktpartner. So eröffnet beispielsweise ein geringes Maß verkaufsfördernder Leistungen bei gleichzeitig höheren Erwartungen von seiten der Marktpartner eine Möglichkeit für Handelsvertretungen, sich in diesem Bereich besonders zu profilieren ${ }^{1}$.

1 Voraussetzung der prognostischen Relevanz ist allerdings, daß die Ausprägungen der Rollenausübung sowie der Erwartungen transparent sind, d.h. die kognitiven Informationen müssen in expliziter Form bekannt sein. Ist dies der Fall, so wird damit gleichzeitig eine Ubereinstimmung von 'role expectations' und 'sent-role' des Rollensenders angenommen (vgl. Abbildung 3). $\mathrm{Zu}$ dieser Diskussion vgl. Biddle, B.J., Roles, Goals, and Value Structures in Organization Research, Hrsg.: W.W. Cooper, New York usw. 1964, S. 150 ff.; Steffenhagen, H., a.a.O., S. 92. Die Problematik der Operationalisierung der kognitiven Informationen für vorliegende Problemstellung behandelt Kapitel B. 2. dieser Arbeit. 
Sowohl aus der theoretischen Diskussion der Rollenanalyse als auch aus ihrer Ubertragung auf den Bereich der Handelsvertretertätigkeit leitet sich damit die Rollenerwartung als zweite wesentliche Komponente einer Rollenanalyse für das Handelsvertreter-Marketing $a b^{1}$.

Rollenerwartungen, d.h. Erwartungen hinsichtlich bestimmter Qualifikationen und Leistungen, sehen sich die Handelsvertretungen dabei in zweifacher Hinsicht gegenüber. Zum einen bestehen derartige Erwartungen auf seiten der vertretenen Unternehmen, zum anderen liegen Erwartungen ebenfalls bei den Kunden vor. Beispielsweise hat ein Hersteller eine bestimmte Vorstellung darüber, wie die Kundenbetreuung durch seine Handelsvertretungen durchgeführt werden sollte (Besuchsfrequenz, Besuchsdauer). In ähnlicher Weise erwarten die Kunden ein bestimmtes Maß an Betreuungsaktivitäten.

Das Zusammenwirken der - möglicherweise unterschiedlichen Rollenerwartungen mit der Rollenausübung von Handelsvertretungen kommt bereits im dualen Modell des Handelsvertreter-Marketing zum Ausdruck. Das Phänomen mehrdeutiger Rollenerwartungen ist für Handelsvertretungen von besonderem Interesse, wenn es darum geht, das gesamte Dienstleistungsangebot $\mathrm{zwischen}$ vertretenen Unternehmen und Kunden zu koordinieren. Eine Rollenanalyse im Han-

1 Die Rollenerwartung deckt sich weitgehend mit dem zuvor diskutierten normativen Rollenkonzept, vgl. Wiswede, G., a.a.O., S. 37 ff.; Thomas, E.J., Biddle, B.J., Basic Concepts for Classifying the Phenomena of Role, a.a.O., S. 26; Mills, T.M., Soziologie der Gruppe, München 1974 , S. $117 \mathrm{ff.}$. 
delsvertreter-Marketing kann daher auf eine Einbeziehung differenzierter Rollenerwartungen der Marktpartner nicht verzichten ${ }^{1}$.

\subsection{Rollenerfüllung von Handelsvertretungen}

Rollenausübung von Handelsvertretungen und Rollenerwartungen der Marktpartner beschreiben zwei wesentliche Komponenten einer Rollenanalyse im Handelsvertreter-Marketing. Damit ist es gelungen, zwei zentrale Elemente des allgemeinen rollenanalytischen Grundmodells für die verhaltenswissenschaftliche Analyse vertikaler Absatzkanalsysteme nutzbar zu machen. Betrachtet man über die mit einer Rollenanalyse erzielbaren Erklärungszusammenhänge hinaus die Gestaltung handelsvertreterspezifischer Marketingmaßnahmen als ein zu lösendes Problem, so erweist sich die Hinzunahme einer weiteren Rollenkomponente als notwendig und sinnvoll.

Das Einwirken der Rollenerwartungen von Mitgliedern eines sozialen Systems auf einen Positionsinhaber führt in Verbindung mit dessen Selbstverständnis im allgemeinen Modell

1 Die hier diskutierte Kombination unterschiedlicher Rollenerwartungen ist in der allgemeinen Rollentheorie als 'role-set' bekannt, vgl. insbesondere Wiswede, G., a.a.O., S. $81 \mathrm{ff.;}$ Merton, R.K., The Role-Set: Problems in Sociological Theory, in: BJS 1957, S. $110 \mathrm{ff.;}$ ders., Instability and Articulation in the Role-set, in: Biddle, B.J., Thomas, E.J., a.a.O., S. $282 \mathrm{ff..}$ Zur Integration des role-set in soziale Systeme vgl. Evan, W.M., Toward a Theory of Inter-Organizational Relations, in: MS 1965, S. $217 \mathrm{ff.}$ 
zu einer bestimmten Verhaltensausprägung des Positionsinhabers ${ }^{1}$. Ubertragen auf den Bereich der Handelsvertretertätigkeit haben demnach die Rollenerwartungen der Marktpartner sowie die Vorstellungen über den eigenen Aufgabenbereich $\mathrm{zu}$ einem realisierten Angebot von Leistungen gefüht. Aus der Sicht der Handelsvertretung wurde dieses Leistungsangebot als Ausdruck ihrer Rollenausübung verstanden. Speziell im Hinblick auf die bereits erwähnte Problemstellung der Konzipierung eines HandelsvertreterMarketing rückt eine weitere Fragestellung in den Mittelpunkt des Interesses.

Hierbei handelt es sich um die Frage, wie die Marktpartner das gegenwärtige Leistungsangebot von Handelsvertretungen sehen, d.h. wie sie die Ausübung der von den Handelsvertretungen übernommenen Rolle wahrnehmen. Zur Abgrenzung von der Rollenausübung aus der sicht der Handelsvertretungen selbst wird die Wahrnehmung der ausgeübten Rolle durch die Marktpartner als Rollenerfüllung bezeichnet. Dieser Terminus umschreibt damit das gegenwärtige Rollenverhalten von Handelsvertretungen aus dem Blickwinkel ihrer Marktpartner ${ }^{2}$.

1 Vgl. insbesondere Katz, D., Kahn, R.L., a.a.O., S. 182f.; Steffenhagen, H., a.a.O., S. 85 f.; Kirsch, W., Entscheidungsprozesse. 3. Band, a.a.0., S. 107.

2 Das in der allgemeinen Rollentheorie diskutierte 'Rollenverhalten' erfährt somit für die vorliegende Problemstellung eine stärkere Differenzierung im Hinblick auf die unterschiedlichen Blickrichtungen der Mitglieder in einem sozialen System. Zum Rollenverhalten vgl. Turner, R.M., a.a.O., S. 391 f.; Thomas, E.J., Biddle, B.J., Basic Concepts for Classifying the Phenomena of Role, a.a.O., S. 41 ff.; Kast, F.E., Rosenzweig, J.E., Organization and Management - A Systems Approach, New York usw. 1970, S. 264; Harvey, D.F., Brown, D.R., An Experimential Approach to Organization Development, Englewood Cliffs 1976 , S. 271 f.. 
Rollenerfüllung (aus der Sicht der Marktpartner) und Rollenausübung (aus der Sicht der Handelsvertretung) müssen dabei nicht notwendigerweise übereinstimmen. So kann beispielsweise ein Handelsvertreter im Rahmen seiner Reisetätigkeit Kundenbesuche absolvieren, die lediglich einer allgemeinen Information und Betreuung dienen. Demgegenüber registriert das vertretene Unternehmen nur solche Besuche, die sich in Form eines Auftrages niederschlagen. Es ist offensichtlich, daß in einem derartigen Fall die Ausübung der Kundenbetreuung durch Handelsvertretung und Hersteller divergierend gesehen wird. Die Rollenerfüllung aus der Sicht der Marktpartner stellt daher bei einer Rollenanalyse im Handelsvertreter-Marketing eine dritte eigenständige Komponente des allgemeinen Rollenkonstruktes dar.

Die bisherigen Ausführungen ließen mehrfach eine Verzahnung der vorgestellten Rollenkomponenten deutlich werden, z.B. die prognostische Relevanz aus der Abweichung von Rollenausübung und Rollenerwartung. Aus diesem Grunde gilt es, im folgenden die Komponenten in ihrem Zusammenwirken zu diskutieren, womit zugleich die Rollenbeziehungen im Handelsvertreter-Marketing transparent werden.

\subsection{Rollenbeziehungen im Handelsvertreter-Marketing}

Rollenausübung, Rollenerfüllung und Rollenerwartung lassen sich in Verknüpfung mit der dualistischen Stellung der Handelsvertretung im Absatzkanal zu einem Modell der Rollenanalyse im Handelsvertreter-Marketing zusammenführen. Dieses Modell bietet durch die explizite Einbeziehung aller relevanten Marktpartner wesentliche Ansatzpunkte, die Rollenbeziehungen im Handelsvertreter-Marketing aufzudecken. 


\subsection{Integration der Rollenkomponenten}

(Rollenmodell des Handelsvertreter-Marketing)

Entsprechend der Stellung einer Handelsvertretung im $\mathrm{Ab}-$ satzkanal muß das Modell einer Rollenanalyse im Handelsvertreter-Marketing drei Betrachtungsebenen aufweisen, wobei jede dieser Ebenen die ihr zugehörigen Rollenkomponenten umfaßt. Abbildung 4 zeigt die drei Ebenen mit den entsprechenden Rollenkomponenten ${ }^{1}$.

Im Mittelpunkt des Modells steht die Handelsvertretung mit einer spezifischen Rollenausübung (1). Auf seiten der Marktpartner wird diese Rollenausübung als Rollenerfüllung wahrgenommen ( $2 \mathrm{a}$ und $2 \mathrm{~b}$ ). Gleichzeitig hegen vertretene Unternehmen und Kunden bestimmte Rollenerwartungen ( $3 a$ und $3 b$ ). Auf der Grundlage dieser fünf bereits im Detail analysierten Rollenkomponenten kristallisiert sich sowohl innerhalb der einzelnen Betrachtungsebenen als auch im Vergleich der Ebenen untereinander ein symmetrisches Beziehungsgefüge zwischen den Marktpartnern heraus.

Die Strukturierung der Rollenkomponenten innerhalb einer Betrachtungsebene läßt sich als horizontale Rollenbeziehung kennzeichnen, die Gegenüberstellung von Komponenten unterschiedlicher Ebenen beschreibt vertikale Rollenbeziehungen. Art und Ausprägungen dieser Beziehungen determinieren das rollenbestimmte Verhalten aller Beteiligten, d.h. die Interaktion der Marktpartner im Absatzkanal. Eine umfassende Analyse der Wechselwirkungen zwischen den einzelnen Rollenkomponenten ist daher unabdingbarer

1 Das vorgestellte Modell konkretisiert somit das allgemeine Rollenmodell im Hinblick auf die spezifische Situation der Handelsvertretung im Absatzkanal. Zum Grundmodell vgl. Katz, D., Kahn, R.L., a.a.O., S. 187 bzw. Kapitel B.1.1 dieser Arbeit. 


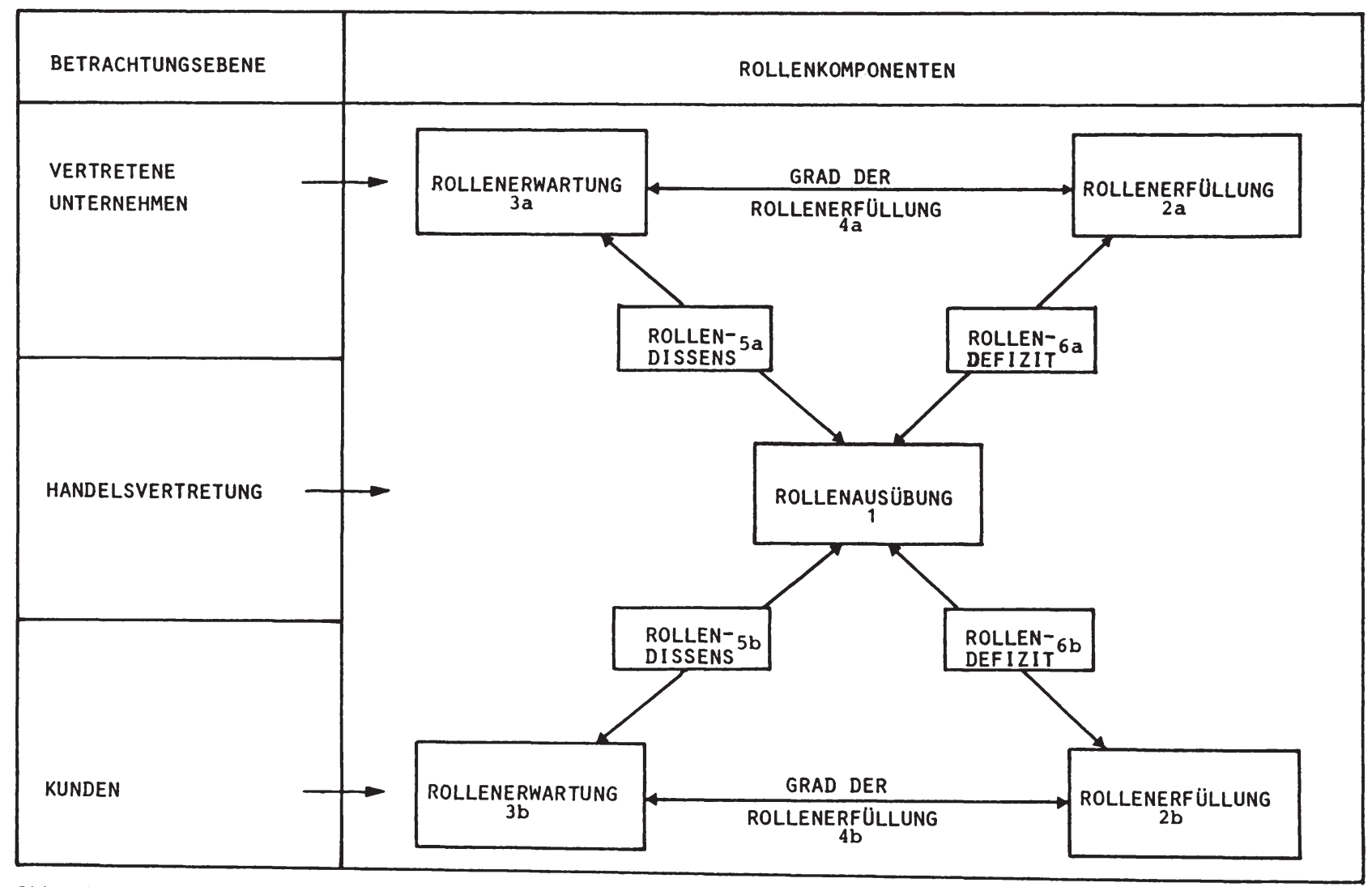

Abb. 4: Rollenmodell des Handelsvertreter-Marketing 
Bestandteil einer Rollenanalyse im Handelsvertreter-Marketing ${ }^{1}$.

\subsection{Horizontale Rollenbeziehungen}

Rollenbeziehungen horizontaler Art werden bei einer separaten Betrachtung der drei Modellebenen deutlich. Bei den Marktpartnern einer Handelsvertretung kann zwischen der Rollenerwartung (3a / 3b) und der Rollenerfüllung $(2 \mathrm{a} / 2 \mathrm{~b})$ eine Diskrepanz auftreten ${ }^{2}$. Beispielsweise registriert ein Hersteller nur in geringem Maße eine Ausweitung des Kundenkreises (niedrige Rollenerfüllung der Handelsvertretung), während seine Erwartungen in Bezug auf die-

1 Neben diesen Interdependenzen aufgrund der Rollenkonstellation unterscheidet man im Beziehungsgefüge sozialer Systeme Macht- und Kommunikationsbeziehungen; vgl. etwa Oeser, O.A., Harary, F., A mathematical model for structure role theory, in: Human Relations 1962 , S. 89 ff.; Wiswede, G., a.a.O., S. 78 f.; Beier, F.J., Stern, L.W., Power in the Channel of Distribution, in: Distribution Channels: Behavioral Dimensions, a.a.O., S. 92 ff.; Grabner, J.R., Rosenberg, L.J., Communication in Distribution Channel Systems, in: Distribution Channels: Behavioral Dimensions, a.a.0., S. $227 \mathrm{ff}$. Die Bedeutung von Zielbeziehungen für die Absatzkanalforschung betonen Meffert, $H$., Vertikales Marketing und Marketingtheorie, in: Steffenhagen, H., a.a.O.,S. 19; Steffenhagen, H., a.a.O., S. $45 \mathrm{f}$.. Für das Handelsvertreter-Marketing liefern diese Beziehungsformen Erklärungsansätze bei der Analyse der Bestimmungsfaktoren von Rollenkomponenten, vgl. Kapitel C. 3. dieser Arbeit.

2 Diese Diskrepanz ist nicht $z u$ verwechseln mit dem IntraRollenkonflikt, der aufgrund der Unvereinbarkeit mehrdeutiger Rollenerwartungen auftreten kann. Vgl. hierzu Wiswede, G., a.a.O., S. 115, Gross, N., McEachern, A.W., Mason, W.S., a.a.O., S. 288; Beispiele belegen March, J.G., Simon,H.A., Organisation und Ind1viduum Wiesbaden 1976, S. 91. 
ses Merkmal deutlich höher liegen (hohe Rollenerwartung an Handelsvertretungen). Dieser Unterschied zwischen Rollenerfüllung und Rollenerwartung kennzeichnet somit, inwieweit eine Handelsvertretung die an ihre Rolle geknüpften Erwartungen gegenwärtig $z u$ erfüllen vermag. Diese bei Herstellern und Kunden gleichermaßen auftretende Diskrepanz wird als Grad der Rollenerfüllung einer Handelsvertretung bezeichnet $(4 \mathrm{a}$ und $4 \mathrm{~b}$ ).

Es ist zu vermuten, daß positive Ausprägungen des Grades der Rollenerfüllung - d.h. relativ geringe Unterschiede zwischen Rollenerfüllung und -erwartung - zu weitgehend harmonischen Beziehungen im Absatzkanal führen. Kennzeichen solcher Beziehungen sind beispielsweise relativ konfliktfreie Räume in Bezug auf den gemeinsamen Berührungspunkt der Marktpartner - das Dienstleistungsangebot der Handelsvertretungen. Im Gegensatz dazu läßt ein niedriger Grad der Rollenerfüllung - d.h. hohe Diskrepanzen zwischen Rollenerfüllung und -erwartung - eher Konflikte in einzelnen Bereichen des Leistungsangebotes erwarten.

Identifikation und Interpretation horizontaler Rollenbeziehungen sind demnach ein weiterer bedeutsamer Untersuchungsbereich einer Rollenanalyse im HandelsvertreterMarketing. Es leuchtet jedoch unmittelbar ein, daß weiterhin die Abgrenzung vertikaler Rollenbeziehungen einen wesentlichen zusätzlichen Erklärungsbeitrag zum Verständnis des gesamten Rollengefüges leistet. Der Grad der Rollenerfüllung (d.h. horizontale Rollenbeziehungen) kann in seinen Auswirkungen auf das Handelsvertreter-Marketing erst dann abschließend beurteilt werden, wenn die Interdependenzen zur Rollenausübung aus der Sicht der Handelsvertretungen - gleichsam als Relativierungsmaßstab - er- 
gänzend herangezogen werden.

\subsection{Vertikale Rollenbeziehungen}

Die Beziehungen zwischen den Modellebenen, d.h. die vertikalen Rollenbeziehungen, weisen eine zweiseitige Struktur auf. Zum einen bestehen Unterschiede zwischen der Rollenausübung und den Rollenerwartungen, zum anderen können Rollenausübung und Rollenerfüllung voneinander abweichen ${ }^{1}$.

Ein vorhandener Unterschied zwischen Rollenausübung und Rollenerwartungen wurde bereits im Hinblick auf seine prognostische Relevanz vorgestellt. Demnach kann eine Handelsvertretung die Rollenerwartungen der Marktpartner als Orientierungsmaßstab für die Gestaltung des zukünftigen Dienstleistungsangebotes heranziehen. Wenn jedoch eine Handelsvertretung eine Anpassung des Dienstleistungsangebotes an die Rollenerwartungen nicht vornehmen kann oder will, so beinhaltet die beschriebene Diskrepanz zugleich ein hohes Maß an Konfliktpotential ${ }^{2}$. Konflikte können dabei

1 Wegen der Zentrierung der vorliegenden Problemstellung auf die Handelsvertretung bleiben weitere Konfliktfelder, die sich aus der Beziehung zwischen Herstellern und Kunden ableiten lassen, unberücksichtigt.

2 Die Unvereinbarkeit unterschiedlicher Rollenerwartungen wird auch Inter-Rollenkonflikt genannt. Vgl. etwa Wiswede, G., a.a.O., S. 115; Banton, M., Roles, a.a.O., S. $151 \mathrm{ff.;}$ Goode, W.J., A Theory of Role Strain, in: Behavioral Science Foundations of Consumer Behavior, Hrsg.: J.B. Cohen, New York usw. 1972, S. 407 ff.. 
sowohl mit den vertretenen Unternehmen (Beispiel Neukundengewinnung) als auch mit den Kunden auftreten (Beispiel Besuchsfrequenz). Diese Art potentieller Konflikte wird für die Rollenanalyse im Handelsvertreter-Marketing als Rollendissens bezeichnet ( $5 \mathrm{a}$ und $5 \mathrm{~b})^{1}$.

In Abgrenzung zum Rollendissens wird die zweite Art möglicher Abweichungen zwischen den Betrachtungsebenen mit dem Terminus Rollendefizit belegt ( $6 \mathrm{a}$ und $6 \mathrm{~b})$. Dieser Begriff umschreibt den Sachverhalt, daß die Wahrnehmungen aller Beteiligten über die gegenwärtige Rolle der Handelsvertretung voneinander abweichen ${ }^{2}$.

Das beschriebene Beispiel aus dem Bereich der Kundenbetreuung verdeutlichte ${ }^{3}$, daß Hersteller und Handelsvertretung aus ihrem subjektiven Blickwinkel jeweils 'richtige' Wahrnehmungen aufweisen. Der Versuch zur Objektivierung dieses Sachverhaltes deckt dagegen auf, daß unterschiedliche Bewertungsmaßstäbe von Hersteller und Handelsvertretung Ursache des Rollendefizits sind. Während die vertretenen Unternehmen die Besuchsfrequenz allein über die Zahl der vermittelten Aufträge feststellen (können), finden bei den Handelsvertretungen auch solche Kundenkontakte Berücksichtigung, die nicht unmittelbar als

1 Zum Begriff des Rollendissens vgl. Thomas, E.J., Biddle, B.J., Basic Concepts for Classifying the Phenomena of Role, a.a.O., S. 33;, Sarbin, T.R., Allen, V.L., a.a.O., S. 504; Krüger, W., a.a.O., S. $390 \mathrm{ff..}$

2 Der Begriff Rollendefizit ist Ausdruck der für die vorliegende Problemstellung konkretisierten allgemeinen Rollenbeziehungen; vgl. Kapitel B. 1.23 dieser Arbeit sowie Kast, F.E., Rosenzweig, J.E., a.a.O., S. 266 f.; Parsons, T., a.a.0., S. $280 \mathrm{ff.}$.

3 Vgl. Kapitel B. 1.23 dieser Arbeit. 
Auftrag faßbar sind. Eine Änderung des Bewertungsmaßstabes einer der Marktpartner führt zu unmittelbaren Auswirkungen auf die wechselseitigen Rollenbeziehungen. So kann etwa ein Hersteller die $\mathrm{Zahl}$ der insgesamt getätigten Kundenbesuche verwenden, wenn ihm von seiten der Handelsvertretung die entsprechenden Informationen zur Verfügung stehen. Ein solchermaßen erhöhter Informationsstand des Herstellers führt zu einer Verminderung des Rollendefizits.

Eine Verringerung des Rollendefizites (6a) zwischen Hersteller und Handelsvertretung beeinflußt - in Verbindung mit einer entsprechend höheren Ausprägung der Rollenerfüllung (2a) - den Grad der Rollenerfüllung in der Wahrnehmung durch den Hersteller (4a). Im vorliegenden Beispiel verringert sich die Diskrepanz zwischen Rollenerwartung (3a) und Rollenerfüllung (2a). Gelingt der Handelsvertretung ein vollständiger Abbau des Rollendefizites (6a), so verbleibt der Rollendissens (5a) als alleinige Bestimmungsgröße konfliktärer Beziehungen im HandelsvertreterMarketing ${ }^{1}$.

Die am Beispiel veranschaulichte Wirkungsweise von Rollendefizit und Rollendissens konnte erhärten, daß die interdependenten Beziehungen horizontaler und vertikaler Art für eine umfassende Rollenanalyse im Handelsvertreter-Marketing unverzichtbar sind. Im folgenden gilt es daher, geeignete Mittel zu finden, die in der Lage sind, Kom-

1 Ein vollständiger Abbau von Rollendefizit und -dissens führt $z u$ einem Rollenkonsens, der jedoch wegen damit verbundener starrer Verhaltensweisen wenig erstrebenswert ist; ein Minimum an Konsens ist dagegen für die Gleichgewichtsfähigkeit eines Distributionssystems unerläßlich; vgl. Steffenhagen, H., a.a.0., S. 95 f.; Tosi, H.L., The Effects of Expectation Levels and Role Consensus on the Buyer-Seller Dyad, in: JoB 1966, S. 518 f.. 
ponenten und Beziehungen im Rollenmodell des Handelsvertreter-Marketing $\mathrm{zu}$ operationalisieren und damit einer empirischen Erfassung zugänglich zu machen.

2. Ansatzpunkte zur Erfassung von Rollen und Rollenbeziehungen im Handelsvertreter-Marketing

Eine empirisch unterstützte Rollenanalyse im Handelsvertreter-Marketing erfordert die Bereitstellung eines geeigneten Instrumentariums zur Erfassung aller Rollenausprägungen sowie rollenbestimmten Verhaltensweisen. Dies ist gleichbedeutend mit der Frage, "welche Verhaltensbereiche im allgemeinen als rollendeterminiert betrachtet werden können ... und wovon die Determiniertheit abhängt" 1 . Eine Beantwortung dieser Frage erfordert in einem ersten Schritt die Generierung bzw. Zusammenstellung rollenprägender Merkmale im Handelsvertreter-Marketing. Die zweite Teilfrage verlangt eine Herausarbeitung von Bestimmungsfaktoren, die ihrerseits auf die Rollen bzw. Rollenbeziehungen im Handelsvertreter-Marketing einwirken.

\subsection{Dienstleistungen von Handelsvertretungen als rollen- prägende Merkmale}

Das Rollenmodell im Handelsvertreter-Marketing (vgl. Abbildung 4) hat wiederholt die Bedeutung der von einer Handelsvertretung angebotenen Dienstleistungen für eine Rollenanalyse herausgestellt. Dies galt insbesondere im Hinblick auf eine differenzierte Betrachtung einzelner Leistungen (z.B. bei der Kundenbetreuung). Bevor jedoch ein differenziertes Dienstleistungsangebot als Grund-

1 steffenhagen, H., a.a.O., s. 89. 
lage einer Rollenanalyse im Handelsvertreter-Marketing herangezogen werden kann, muß als Voraussetzung hierfür zunächst die grundsätzliche Eignung derartiger Funktionsmerkmale zur Operationalisierung von Rollen im vertikalen Marketing überprüft werden.

\subsection{Die Eignung von Dienstleistungen zur Erfassung von Rollen}

Die Abgrenzung rollenprägender Bereiche im Handelsvertreter-Marketing orientiert sich zweckmäßigerweise an dem Schwerpunkt der unternehmerischen Betätigung von Handelsvertretungen ${ }^{1}$. Dieser Schwerpunkt bzw. die Domäne dokumentiert sich allgemein in Verhaltensweisen, die für eine Handelsvertretung charakteristisch sind. Wesentliche Bestandteile einer Domäne sind allgemein das Produktangebot (range of products), der Kundenstamm bzw. das Absatzgebiet (population served) sowie die Serviceleistungen (services rendered, functions performed). Die Position der Handelsvertretung im Absatzkanal führt demnach $z u$ bestimmten - domänenspezifischen - Ausprägungen ihres Leistungsangebotes, das damit zugleich ihr gesamtes Tätigkeitsfeld, d.h. ihre Domäne im Absatzkanal abgrenzt ${ }^{2}$.

Mit diesem Angebot bestimmter Leistungen - bzw. dem NichtAnbieten von Leistungen - wird zugleich die Verbindung $z u$ den vor- und nachgelagerten Absatzstufen hergestellt.

1 Zur allgemeinen Diskussion rollenprägender Bereiche in Absatzkanälen vgl. Steffenhagen, H., a.a.O., S. $89 \mathrm{f}$. sowie Stern, L.W., Heskett, J.C., a.a.O., S. 293 f..

2 Zur Domäne und ihren vorgestellten Teilbereichen vgl. Thompson, J.D., Organizations in Action, New York usw. 1967, S. 26 f.. 
Rechnet z.B. eine Handelsvertretung Leistungen im Bereich der Warenanlieferung nicht zu ihrer Domäne, müssen andere Elemente des Absatzkanals diese Leistungen übernehmen. Für den Fall, daß ein Hersteller logistische Aufgabenstellungen aus seinem Unternehmen ausgliedern will, ergeben sich unmittelbare Konsequenzen auf die Interaktion zwischen Hersteller und Handelsvertretung.

In dem Beispiel wird deutlich, daß unterschiedliche Vorstellungs- bzw. Erwartungshaltungen hinsichtlich bestimmter Leistungen Ansatzpunkte für Konflikte aufweisen. Gleichzeitig belegt das Beispiel die enge Verzahnung von Domänen im Absatzkanal. Die von einer Handelsvertretung abgegrenzte Domäne stellt damit gleichsam das Bindeglied zwischen den Domänen der Hersteller und Kunden dar. Wegen der hier erkennbaren Bedeutung der Ausprägungen von Domänen bei den Marktpartnern für das gesamte Beziehungsgefüge im Absatzkanal wird den genannten Merkmalen einer Domäne - d.h. den übernommenen Leistungen - eine in hohem Maße rollenbildende Wirkung zugeschrieben ${ }^{1}$. Das Kernstück von Rollen und Rollenbeziehungen bilden demnach konkrete Funktionen bzw. Funktionsbündel. Ubertragen auf die Rollenanalyse im Handelsvertreter-Marketing bedeutet dies, daß das Dienstleistungsangebot von Handelsvertretungen als dominierend rollenprägender Bereich angesehen werden muß.

Die rollenprägende Funktion des Dienstleistungsangebotes bringt zum Ausdruck, daß sich eine Rollenanalyse im Handelsvertreter-Marketing an den einzelnen Dienstleistungen orientieren kann. Damit ist als nächstes zu klären, wie die einzelnen Elemente des Dienstleistungsangebotes einer Rollenanalyse zugänglich gemacht werden können. Fragen der Operationalisierung und insbesondere rollenbezogenen

1 Vgl. Steffenhagen, H., a.a.O., S. 89. 
Erfassung von Dienstleistungen stehen dabei im Vordergrund.

\subsection{Operationalisierung von Dienstleistungen für eine Rollenanalyse}

Das grundlegende Problem der empirischen Erfassung von Rollen begründet sich aus dem vorwiegend kognitiven Charakter dieses Konstrukts ${ }^{1}$. Eine Rollenanalyse im Handelsvertreter-Marketing - verstanden als Informationsgrundlage zur Konzipierung bzw. Weiterentwicklung von Marketingmaßnahmen - erfordert eine offenlegung der kognitiven Rolleninhalte. Da sich das Dienstleistungsangebot von Handelsvertretungen als dominierend rollenprägend im $\mathrm{Ab}-$ satzkanal herausgestellt hat, konkretisiert sich das Problem der empirischen Erfassung von Rollen im Absatzkanal auf die Frage: Wie lassen sich Rollen und Rollenbeziehungen im Handelsvertreter-Marketing auf der Basis des Dienstleistungsangebotes der Handelsvertretungen transparent machen?

Die bisherige empirische Erforschung von Rollen verwendete unterschiedliche Techniken zur Erfassung. Beobachtungen sowie Laborexperimente lassen sich als zwei wesentliche Meßtechniken ausmachen ${ }^{2}$. Daneben hat das Instrument der Befragung vielfach Anwendung gefunden, wobei jedoch aus-

1 Vgl. Kirsch, W., a.a.O., S. 102 sowie Kapitel B. 1.1 dieser Arbeit.

2 Vgl. Popitz, H., a.a.O., S. 25 f.; Beispiele hierzu liegen insbesondere aus dem Bereich der Organisationsforschung vor. Vgl. insbesondere Katz, D., Kahn, R.L., a.a.O., S. $188 \mathrm{ff.}$ sowie March, J.G., Simon, H.A., a.a.O., S. 91; Hoffmann, F., Entwicklung der Organisationsforschung, Wiesbaden 1973, S. 94 f.. 
führliche Erhebungen unter realen Bedingungen eher selten Verwendung fanden ${ }^{1}$. In der Distributionsforschung wurde der empirischen Rollenanalyse vergleichsweise wenig Beachtung zuteil ${ }^{2}$. Dies erstaunt angesichts der Feststellung, da $\beta$ bereits in einem relativ frühen Forschungsstadium die Befragung dazu verwendet wurde, Selbstbild, Fremdbild sowie projektives Fremdbild bestimmter sozialer Rollen zueinander in Beziehung $z u$ setzen ${ }^{3}$. Die bisher genannten Untersuchungsbereiche Selbstbild, Fremdbild sowie projektives Fremdbild weisen ein hohes Maß an Ubbereinstimmung mit den Elementen des Rollenmodells für das Handelsvertreter-Marketing auf ${ }^{4}$. Es liegt daher nahe, diesem Forschungsansatz zu folgen und die Befragung als wesentliches Instrument einer Rollenanalyse im Handelsvertreter-Marketing heranzuziehen.

Für die konkrete inhaltliche Ausgestaltung einer Befragung zur Rollenanalyse des Handelsvertreter-Marketing deckt der genannte allgemeine Forschungsansatz ebenfalls Möglichkeiten auf. Selbstbild und Fremdbild einer Rolle lassen sich für das Handelsvertreter-Marketing als Selbsteinschätzung der eigenen Rollenausübung bzw. Fremdeinschätzung durch die Marktpartner hinsichtlich Rollenerfüllung und

1 Vgl. Thomas, E.J., Biddle, B.J., The Nature and History of Role Theory, in: Role Theory: Concepts and Research, a.a.0., S. 16 .

2 Bekannt wurde in diesem Zusammenhang die studie von Ridgeway, bei der aus sekundärstatistischem Material Rollenbeziehungen in Franchisesystemen analysiert wurden. Vgl. Ridgeway, V., Administration of Manufacturer-DealerSystems, in: ASQ 1957, S. $464 \mathrm{ff.;}$ Steffenhagen, H., a.a.O., s. $91 \mathrm{f}$..

$3 \mathrm{Vgl}$. Rommetveit, R.R., Social Norms and Roles, Explorations in the Psychology of Enduring Social Pressures, Oslo-Minneapolis 1955, S. 123 ff.; Popitz, M., a.a.O., S. 26 .

$4 \mathrm{Vgl}$. Abbildung 4 dieser Arbeit. 
-erwartung charakterisieren ${ }^{1}$. Die Erfassung solcher Einschätzungen, d.h. Vorstellungen und Meinungen über ein objekt (hier: Handelsvertretungen) ist in der produktbezogenen Marketingforschung vielfach verbreitet ${ }^{2}$ und hat auch in der Erforschung nicht-kommerzieller Marketingproblemstellungen seine Eignung unter Beweis gestellt ${ }^{3}$.

Aus den genannten Gründen liegt es nahe, den Grundgedanken vorliegender empirischer Ansätze aufzugreifen und auf das Handelsvertreter-Marketing zu übertragen. Die Rollenanalyse im Handelsvertreter-Marketing kann daher über die Erfassung von Selbst- und Fremdeinschätzungen vorgenommen werden. In Verbindung mit dem Dienstleistungsangebot als rollenprägendem Merkmal ist somit die Beurteilung des Dienstleistungsangebotes von Handelsvertretungen zentraler Untersuchungsbereich einer Rollenanalyse im Handelsvertreter-Marketing.

Nachdem die grundsätzliche Vorgehensweise einer Rollenanalyse im Handelsvertreter-Marketing abgegrenzt werden konnte - d.h. die Frage nach der Eignung von Dienstleistun-

1 Vgl. Sarbin, T.R., Turner, R.H., a.a.O., S. 546; diese stellen besonders die Fremdbeurteilung (behaviors being noted by observers who may use behavior rating scales) als zentrale Meßmöglichkeit heraus.

2 Einen Uberblick der Erfassung subjektiver Einschätzungen bzw. Einstellungen geben Meffert, H., Marketing, a.a.O., S. $121 \mathrm{ff}$. und S. $178 \mathrm{ff}$. sowie Kroeber-Riel, W., Konsumentenverhalten, 2. Aufl., München 1980 .

$3 \mathrm{Vgl}$. hierzu Kook, W. , Einstellungen zur Universität, Operationalisierung und empirische Prüfung verhaltenswissenschaftlicher Hypothesen am Beispiel der Stadtbevölkerung von Münster, Frankfurt 1981. 
gen positiv beantwortet sowie Möglichkeiten ihrer Operationalisierung festgestellt wurden - muß sich das weitere Interesse auf eine Differenzierung einzelner Dienstleistungen konzentrieren, die in eine Rollenanalyse Eingang finden sollen.

\subsection{Kennzeichnung der Dienstleistungsbereiche von Han- delsvertretungen}

Das gesamte Dienstleistungsangebot von Handelsvertretungen beinhaltet eine Palette vielfältiger Einzelleistungen. Der Versuch einer allgemeinen strukturierung dieser Leistungen wird erschwert durch die Tatsache, daß Handelsvertretungen in allen Wirtschaftsbereichen tätig sind ${ }^{1}$. Branchenusancen sowie produkt- und situationsabhängige Einflüsse (z.B. regionale Gegebenheiten) führen zu einer jeweils angepaßten Dienstleistungsstruktur.

Der Generierung einer umfassenden Zusammenstellung von Handelsvertreterleistungen steht zudem eine in der Literatur vergleichsweise wenig ausgeprägte Diskussion über die Handelsvertretung als Dienstleistungsunternehmen entgegen $^{2}$. Die vorwiegend funktionsorientierten Beiträge greifen zumeist einzelne Teilaspekte auf, ohne die Einzelleistungen zu einem gesamten Dienstleistungsbündel zusammenzufügen. Letztlich ist es aber gerade die Gruppierung bestimmter Dienstleistungen, die auf die Marktpartner einwirkt bzw. von diesen gewünscht oder gefordert wird.

1 Vgl. hierzu etwa die Ergebnisse amtlicher Erhebungen: Statistisches Bundesamt (Hrsg.), Fachserie F., Großund Einzelhandel-Gastgewerbe-Fremdenverkehr, Handelsund Gaststättenzählung 1968, III. Handelsvermittlung, Stuttgart-Mainz 1968.

2 Vgl. Meffert, H., Kimmeskamp, G., a.a.0., S. 2. 
Für eine Rollenanalyse im Handelsvertreter-Marketing ist es unerläßlich, die Vielfalt einzelner Funktionen und Leistungen marketingorientiert zu strukturieren. Nur die Einbeziehung eines umfassenden Leistungskatalogs ermöglicht es, die Antezedensbedingungen herauszuarbeiten, die für eine Handelsvertretung gleichsam als Richtschnur zur Uberprüfung und Anpassung ihres Dienstleistungsangebotes und damit zur dynamischen Weiterentwicklung ihrer Rolle im Absatzkanal dienen können.

Bei aller Heterogenität vorliegender Forschungsansätze ${ }^{1}$ läßt sich das Mosaik der dabei verwendeten Leistungsmerkmale $z u$ einem Grundraster eines Dienstleistungsprogramms zusammenfügen, das bis zu einer empirischen Validierung als Arbeitsunterlage dienen kann. Ausgehend von den Basisinstrumenten des Marketing läßt sich für Handelsvertretungen ein Kern von Dienstleistungen abgrenzen. Das "Produkt" eines Handelsvertretungsunternehmens - seine Grundausstattung - bilden das Vertretungsprogramm (d.h. die Zusammensetzung der Produktpalette mit unterschiedlichen Herstellern), die Kundenbetreuung in einem abgegrenzten Gebiet sowie die generelle Leistungsbereitstellung in Form von Ausstattung und Organisation der Handelsvertretung ${ }^{2}$.

1 Diese Heterogenität begründet sich vorwiegend aus den jeweils unterschiedlichen Blickrichtungen. So werden beispielsweise die Leistungen von Handelsvertretungen im Zusammenhang mit der Fragestellung eines optimalen $\mathrm{Ab}-$ satzweges aus Herstellersicht diskutiert; andere Beiträge betonen den Dienstleistungscharakter des Handelsunternehmens. Vgl. beispielhaft Meffert, H., Marketing, a.a.O., S. $398 \mathrm{ff.;}$ Gutenberg, E., Grundlagen der Betriebswirtschaftslehre, Zweiter Band, Der Absatz, 15. Aufl., Berlin-Heidelberg-New York 1976, S. 116 f.; Ahlert, D., Grundzüge des Marketing, Düsseldorf 1980, S. $138 \mathrm{ff}$.; Voss, M. (Hrsg.), Handelsvertreter-Handbuch, München 1979, S. 2 f..

2 Vgl. Meffert, H., Kimmeskamp, G., a.a.O., S. 8 f.. 
Ferner sind Informationsaktivitäten als eigenständige Leistungskomponente festzustellen. Maßnahmen aus den Instrumentebereichen Kontrahierung und Distribution runden die Grundausstattung ab. Darüber hinaus ist eine Vielzahl weiterer Zusatzleistungen zu verzeichnen, die nach Art und Umfang stark situations- bzw. branchenspezifisch ausgeprägt sind.

Grundausstattung und Zusatzleistungen sind mit ihren differenzierten Ausprägungen bzw. Beispielen in Abbildung 5 zusammengefaßt. Auf eine detaillierte Vorstellung einzelner Leistungen soll an dieser Stelle zugunsten einer umfassenderen Diskussion im Rahmen der empirischen Untersuchung verzichtet werden ${ }^{1}$.

Die vorgestellten Merkmale des Dienstleistungsangebotes von Handelsvertretungen stellen den Kern der angestrebten Rollenanalyse dar. Im Hinblick auf die angestrebte empirische

$1 \mathrm{Vgl}$. hierzu die empirische Utberprüfung der Dienstleistungsgruppierung in Kapitel C. 2. sowie die Diskussion der Ausprägungen und Bestimmungsfaktoren von Rollen in C. 3..

2 Quellen zu Abbildung 5:

zentrale Quellen: Meffert 1976, S. 135 ff.; Kimmeskamp 1979 , S. 27 ff.; Meffert und Kimmeskamp 1980, S. 8 ff.; Schröder 1978, S. $14 \mathrm{ff.;}$ Voss 1978, S. $1434 \mathrm{ff.;}$ Voss (Hrsg.) 1979 .

Weitere Quellen: Bachmann 1963; Batzer und Meyerhöfer 1969; Batzer und Meyerhöfer 1970; Baumann 1966; Bethge 1977; Bobrow 1976; Briechle 1965; Gross 1966; Kimmeskamp 1980; Koinecke 1973; Koinecke 1974; Lampe 1962; Schenk, Spannagel, Wölk 1974; Schildmann 1969; Sibley, Teas 1979; Siekamp 1953; Treptow 1972; Voss 1970; Weber 1973; Willas 1975 . 


\section{Grundausstattung}

- Ausstattung und Organisation der Handelsvertretung

- technische Ausstattung

- Erreichbarkeit

- Kundenkartei

- Mitarbeiterqualifikation

- Kundenbetreuung

- Kundenpf lege

- Kundenstrukturanalysen

- Kundenkreiserweiterung

- Reklamationsbearbeitung

- Vertretungsprogramm

- Gestaltung des eigenen Programms

- Zusammensetzung nach Marktanalysen

- Konzentration auf eine Produktgruppe

- Ergänzung der verschiedenen Herstellersortimente

- Mitgestaltung des Herstellersortimentes

- Mitwirkung bei neuen Produkten

- Mitwirkung bei der Sortimentsgestaltung

\section{Information}

- Informationsabgabe an Hersteller und Kunden

- Auswertung der Informationen von Herstellern und Kunden

\section{Kontrahierung}

- kundenbezogen

- Möglichkeit der Preisgestaltung

- Möglichkelt der Konditionenvereinbarung

- herstellerbezogen

- Kalkulation der Provisionssätze

- Vergütung zusätzlicher Leistungen

\section{Distribution}

- Lagerhaltung

- Auslieferung

\section{Zusatzleistungen}

- Kundenschulungen

- Hausmessen beim Kunden

- Sonderverkäufe / Werbeaktionen

- Displayaufstellung / Regalpflege

- Eigene Musterschauen

- Messeteilnahme bel Herstellern

Abb. 5: Struktur des Dienstleistungsangebotes von Handelsvertretungen (mit Beispielen) 
Ausrichtung der Untersuchung sind diese Leistungsmerkmale als abhängige Variable zu bezeichnen. Eine der zentralen Programmfragen dieser Arbeit versucht, die Bestimmungsfaktoren von Rollen und Rollenbezeichnungen im Handelsvertreter-Marketing $\mathrm{zu}$ ergründen. Diese Bestimmungsfaktoren bilden demnach die unabhängigen Variablen der Untersuchung. Erst die Einbeziehung dieser Determinanten von Rollen versetzt in die Lage, differenzierte Aussagen sowie Implikationen für das Handelsvertreter-Marketing abzuleiten.

\subsection{Bestimmungsfaktoren von Rollen und Rollenbeziehungen}

Bei der Frage, welche Faktoren die Rollen und Rollenbeziehungen im Absatzkanal determinieren, sind entsprechend dem Rollenmodell im Handelsvertreter-Marketing (vgl. Abbildung $4)$ drei Betrachtungsebenen zu unterscheiden. Die Ausgangsfragestellung ist im Hinblick auf die Ebene zu präzisieren, auf die jeweils Bezug genommen wird; d.h. für Handelsvertretungen, Hersteller und Kunden sind geeignete Determinanten abzugrenzen.

Betrachtet man es als übergeordnete Zwecksetzung einer empirisch gestützten Rollenanalyse, über die Offenlegung von Rollen und Rollenbeziehungen im Absatzkanal Schlußfolgerungen für handelsvertreterspezifische Marketingstrategien zu ermöglichen, lassen sich folgende generelle Anforderungen formulieren, denen die einzubeziehenden Merkmale genügen müssen ${ }^{1}$ :

1 Der Anforderungskatalog basiert auf den im Rahmen der Marktsegmentierung verwendeten Kriterien; vgl. etwa Meffert, H., Marketing, a.a.O., S. 214 f.; Gröne,A., Marktsegmentierung bei Investitionsgütern,Wiesbaden 1977,s.41 ff.; Schmidt-Brăkling, R., Zielgruppenbestimmung mit Hilfe von Einstellungsskalen - Ein Beitrag zur Marktsegmentierung, Diss. Münster 1973, S. 7 f.; Kotler, P., Marketing Management - Analyse,Planung und Kontrolle, Stuttgart 1974,s. $164 \mathrm{f}$.. 
(1) Die Merkmale müssen mit der Rolle einer Handelsvertretung im Absatzkanal - d.h. mit dem Dienstleistungsangebot - in Verbindung stehen ${ }^{1}$.

(2) Aus der Verbindung der Merkmale mit dem Dienstleistungsangebot müssen sich handelsvertreterspezifische Maßnahmen ableiten lassen.

(3) Die Merkmale müssen mit vorhandenen Methoden mėßbar sein.

(4) Die Merkmale - und damit letztlich die daraus abgeleiteten Schlußfolgerungen - sollen über einen längeren Zeitraum aussagefähig bleiben.

Im folgenden wird für die drei Ebenen des Rollenmodells im Handelsvertreter-Marketing aufgezeigt, welche Kriterien nach Maßgabe der vorgestellten Anforderungen als Bestimmungsfaktoren - d.h. als unabhängige Variable - der Untersuchung gelten können.

\subsection{Merkmale der Handelsvertretungen}

Betrachtet man zunächst die Handelsvertretung als Kernelement des Rollenmodells, konzentriert sich die Ableitung relevanter Bestimmungsfaktoren auf die Frage, welche Merkmale die Rollenausübung von Handelsvertretungen determinieren.

Das von einer Handelsvertretung angebotene Dienstleistungsprogramm wird durch eine Reihe von Faktoren beeinflußt, die

1 Zur Konkretisierung der Rolle einer Handelsvertretung durch die von ihr angebotenen Dienstleistungen vgl. Kapitel B.2.11 dieser Arbeit. 
in der Handelsvertretung selbst bzw. ihrem situativen Umfeld ihren Ursprung haben. So leuchtet unmittelbar ein, daß bestimmte Leistungen nur in manchen Wirtschaftsbereichen anzutreffen sind, beispielsweise Zusatzleistungen wie Regalpflege oder Musterschauen nur bei Konsumgütern, nicht dagegen im Investitionsgüterbereich. In ähnlicher Weise determiniert die Zahl der in einer Handelsvertretung tätigen Personen, wie umfangreich das insgesamt angebotene Dienstleistungsprogramm sein kann.

Abbildung 6 umfaßt eine Zusammenstellung der bedeutsamsten Merkmale, die mit der Ausübung der Rolle von Handelsvertretungen in Beziehung stehen ${ }^{1}$. Als zwei wesentliche Determinantengruppen lassen sich unternehmens- und personenbezogene Einflußgrößen feststellen. Unternehmensbezogene Merkmale beziehen sich auf die Handelsvertretung als Organisation. Hier bilden Branchenzugehörigkeit, Größe des Unternehmens, Vertretungsangebot, Kundenkreis und Standort die wesentlichen Merkmalsgruppierungen. Personenbezogene Merkmale zielen auf die Person des Handelsvertreters, d.h. den Inhaber des Unternehmens, und umfassen Merkmale demographischer Art, die auf das Leistungsangebot hinwirken (z.B. Ausbildung sowie Dauer der Berufstätigkeit) ${ }^{2}$.

1 Die Zusammenstellung der Merkmale orientiert sich an den generellen Kriterien einer Markterfassung, die durch handelsspezifische Merkmale ergänzt wurden. Vgl. Meffert, H., Marketing, a.a.O., S. 215 ff.; Ahlert, D., Grundzüge des Marketing, a.a.0., S. 59 f.; Gröne, A., Marktsegmentierung bei Investitionsgütern, a.a.0., S. 14; Frank, R.E., Massy, W.F., Wind, Y., Market Segmentation, Englewood Cliffs 1972, S. 29 ff.; Forschungsverband für den Handelsvertreter- und Handelsmaklerberuf (Hrsg.), CDHStatistik 1978, Köln 1979 (unveröffentlicht); Collins,R.H., Manufacturers' Representatives for Small Firms, in: JoSBM 1978, S. 13 ff..

2 Auf eine detaillierte Diskussion der unabhängigen Merkmale wird an dieser Stelle zugunsten einer anschaulicheren Diskussion im Rahmen der empirischen Untersuchung verzichtet; vgl. Kapitel C dieser Arbeit. Die diskutierten Zusammenhänge über den Einfluß einzelner Variablen bzw. Merkmalsgruopen werden im Uberblick mit den beiden anderen Modellebenen (Hersteller-Kunden) aufgegriffen; vgl. Kapitel B.3. dieser Arbeit. 


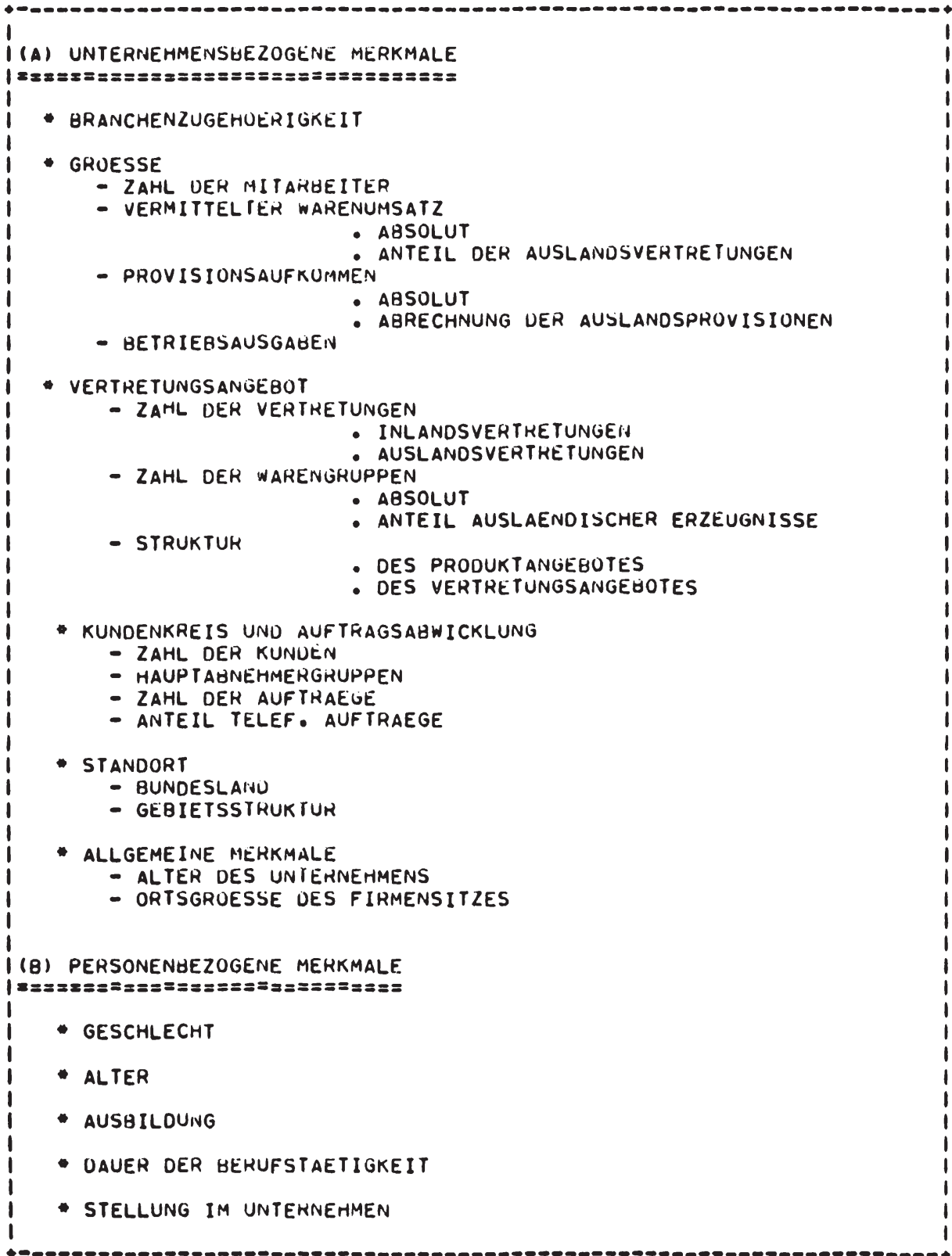




\section{22 Merkmale der vertretenen Unternehmen}

Auf seiten der vertretenen Unternehmen stehen den Handelsvertretungen vorwiegend institutionelle Entscheidungsträger gegenüber. Diese weisen - im Vergleich zur Individualentscheidung eines Konsumenten ${ }^{1}$ - ein komplexeres Entscheidungsverhalten auf $^{2}$. Eine Handelsvertretung, die z.B. ihre Rollenausübung an den Erwartungen dieser Entscheidungsinstitutionen orientieren will, muß daher die relevanten Merkmale kennen, die aufgrund des organisationalen Charakters der Marktpartner einen Einfluß auf die Beurteilung von Rollenerfüllung und auf deren Rollenerwartungen haben.

In Anlehnung an Modelle organisationalen Verhaltens ${ }^{3}$ lassen sich für die vertretenen Unternehmen von Handelsvertretungen die in Abbildung 7 aufgefuhrten Bestimmungsfaktoren abgrenzen ${ }^{4}$.

1 Schwerpunkt der bisherigen Forschung war die Analyse individueller Verhaltensweisen, vgl. etwa Meffert, H., Modelle des Käuferverhaltens und ihr Aussagewert für das Marketing, in: ZfgSt 1971,S.326 ff.; Engel,J.F., Kollat,D.T., Blackwell, K.D., Consumer Behavior, Hinsdale 1978. Erst in jüngerer Zeit diffundiert Marketing in Bereiche organisationaler Verhaltensweisen,z.B. den Investitionsgütersektor; vgl. Meffert, H., Perspektiven des Marketing in den 8oer Jahren, in: DBW 1980,S.59; Gröne, A., a.a.O., S. 8.

2 Vgl. Meffert, H., Marketing, a.a.O., S. 140; Gröne,A., a.a.0., S. 9 .

$3 \mathrm{Vgl}$. insbesondere Webster, F.E.,Wind,Y., Organizational Buying Behavior, Englewood Cliffs 1972; dieselben, A General Model for Understanding Organizational Buying Behavior, in: JoM 1972,S.12 ff.; Zinser,W., Der Absatz von Investitionsgütern. Ein Beitrag zur Bestimmung von Marktsegmenten mit Hilfe von Einstellungsdaten, Berlin 1978,S.42ff.; Meffert,H., Produktivgüter-Marketingforschung im System des Marketing,in: Der Markt 1974,S.6 ff.; Gröne,A., a.a.O.,s.8ff..

4 Der Ansatz von We bster/Wind analysiert industrielles bzw. organisationales Kaufverhalten. Wenn man die sich aus einer Rollenanalyse ergebenden Entscheidungskonsequenzen in die Betrachtung einbezieht(z.B. Ablösung von Handelsvertretern wegen mangelnder Rollenerfüllung), so wird die Eignung der Ubertragbarkeit dieses Ansatzes deutlich.Die Entscheidung für oder gegen Handelsvertreter bzw. Austausch von Handelsvertretern unterliegt organisatorischen Prozessen, die in ihren Bestimmungsfaktoren dem Modellansatz ähnlich sind. 
(A) UNTEANEMmENSHEZOGENE MERKMALE

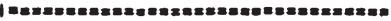

1. Demugraphische merimale

- hetriegstyp

- branche

- BRANCHENZUGEHUEHIGKEI

- UMFAMg des pRUUUKTANgebotes

- STRUKTUA UES PNUDUKTANGEBOTES

- EIINE/MEMRERE WAHENGRUPPEN

- mauptahnehmergrupPen ANTEIL AUSL. ERZEUGNISSE

\section{- groesse}

- zahl der ueschaeftigten

- uMSATz

- absulut

- exportanteil

- standoht

- bunueslano

- OATSGROESSE UES FIRMENSITZES

- ANZAML DEh ZWE IGHERKE

- allgemeine merkmale

- REChTSFUKm

- alter DES UNTEHNEMMENS

2. VERTRIEBSBEZUGENE MERKMALE

- aRt des VeRtadegsSYSTEMS

- groesse der ausat lungane

- ANZAML DEH HANDELSVERTRETER

- INLANO

- anzarl ven reiseinoen

- bedeuturag ver agsatzorgane

- SORTIMEntsanteile

VON HV

VON A

- uMSATzanteILE

- von hi

- von R

- AUS DIREKTGESCHAEFTEN

- DAUER dek zUSAMAENaReEIT

- kriterien oer vehthiEgSorganisation

- BISHERIGE EINTEILUNG (RAEUIAL./KUNOENBE2./PRODUKTBEZ.)

- HEI DEN HANDELSVERTHETERN

- eRsatz Vun mandellsvertRetern

BEI DEN REISENOEN

- KONTAKTAUf NAMME hIT NEUEM manUELSVERTHETER

- logistische mehKmale

- zaml oeh laEver

- LAGEKhal TER

- zaml DEh hV-laEgeh

(B) PERSONENAEZ OGENE mEHKMALE

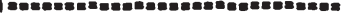

1. DEMOGHAPHISCME MEHKMALE

- geschlecht

- ALTER

- AUSAILDUNG

- stellung im vivtemnenmen

- dauer deh untermemmenszugenoerigkeit

- dauer der positiuirsausuebung

2. PERSOENLICME LUSAMmENAKBEIT MIT hV

- anzaml der pemsoemlicmen rontakte

- antejl der ameejtSzejt

AB8. 78 HESTIMMUNGSFAKTUHEN VON ROLLENEHFUELLUNG UNO -EHWARTUNG QE \ DEN VEHTRETEINEH UNTEANEMAEN 
Eine generelle Unterteilung der Bestimmungsfaktoren trennt deutlich unternehmensbezogene Merkmale von den personenorientierten Variablen. Beide Merkmalsgruppen umfassen allgemeine demographische Merkmale, die das vertretene Unternehmen (z.B. Betriebstyp, Größe, Standort) bzw. die mit den Handelsvertretungen unmittelbar in Kontakt stehende Person kennzeichnen (z.B. Alter, Dauer der Unternehmenszugehörigkeit). Daneben weisen sowohl unternehmens- als auch personenbezogene Merkmale eine Variablengruppe auf, die sich aus der unmittelbaren Interaktion Hersteller-Handelsvertretung begründet.

Bei den unternehmensbezogenen Merkmalen sind dies Faktoren, die sich aus dem konkreten organisatorischen Aufbau des Vertriebs ergeben. Als wesentliche Merkmale sind hier die Art des Vertriebssystems (nur Handelsvertreter oder gemischte Systeme), die Größe der Absatzorgane sowie Kriterien der Vertriebsorganisation und deren weitere Entwicklung (z.B. Austausch von Handelsvertretern durch Reisende) zu nennen. Auf der Ebene personaler Merkmale beschreiben Kriterien der persönlichen Zusammenarbeit zwischen Handelsvertreter und Kontaktpartner beim Hersteller eher die ablauforganisatorische Seite der Interaktion ${ }^{1}$.

\subsection{Merkmale des Kundenmarktes}

Auf der dritten Ebene des Rollenmodells stehen den Handelsvertretungen mit den Kunden ebenfalls vorwiegend orga-

1 Die Zusammenstellung handelsvertreterspezifischer Bestimmungsfaktoren auf Herstellerebene stützt sich neben einer literaturgestützten Vorgehensweise auf explorative Voruntersuchungen; vgl. hierzu Kimmeskamp, G., Tätigkeitsbereiche und Dienstleistungsangebot von Handelsvertretungen, a.a.0., S. 1 insbes. Anmerkung 2 . 
nisatorische Entscheidungseinheiten gegenüber. Die Bestimmungsfaktoren der Rollenerfüllung und Rollenerwartung und damit des Dienstleistungsangebotes von Handelsvertretungen - lassen sich unmittelbar aus den entsprechenden verhaltenswissenschaftlichen Ansätzen ableiten ${ }^{1}$. Auch hier ist - wie bei den Herstellern - eine handelsvertreterspezifische Anpassung einzelner Merkmale angezeigt.

Abbildung 8 gibt einen Uberblick der Bestimmungsfaktoren, die auf seiten des Kundenmarktes einen Einfluß auf die Beurteilung der Rollenerfüllung und die Rollenerwartungen des Abnehmerkreises zu Handelsvertretungen haben. Neben allgemeinen Merkmalen demographischer Art (unternehmens- und personenbezogen) rücken beim Kundenmarkt einkaufsbezogene Variablen in den Mittelpunkt der Betrachtung.

Hier sind zum einem die generellen Bezugsquellen von Bedeutung, über die die einzelnen Kunden jeweils ihre Erzeugnisse beziehen. Merkmale dieser Art können z.B. Auskunft geben über das Ausma $B$ der über Handelsvertreter bezogenen Waren im Vergleich zu anderen Absatzorganen (Reisende, Einkaufszentrale) und den daraus resultierenden Anforderungen an Handelsvertretungen. Darüber hinaus geben Merkmale der Einkaufsorganisation Hinweise über die aufbau- und ablauforganisatorischen Gegebenheiten des Kundenmarktes. Die Strukturierung der Einkaufsabteilung (z.B. hersteller- oder produktorientiert), die Zahl der Einkäufer sowie die Art der Einkaufsentscheidung (individuell, kollektiv) veranschaulichen diese Merkmale.

1 Es finden daher die bereits bei den vertretenen Unternehmen diskutierten Ansätze auch beim Kundenmarkt Berücksichtigung; vgl. Webster, F.E., Wind, Y., Organizational Buying Behavior, a.a.0., S. 33 ff.; Gröne, A., a.a.0., s. 14 . 


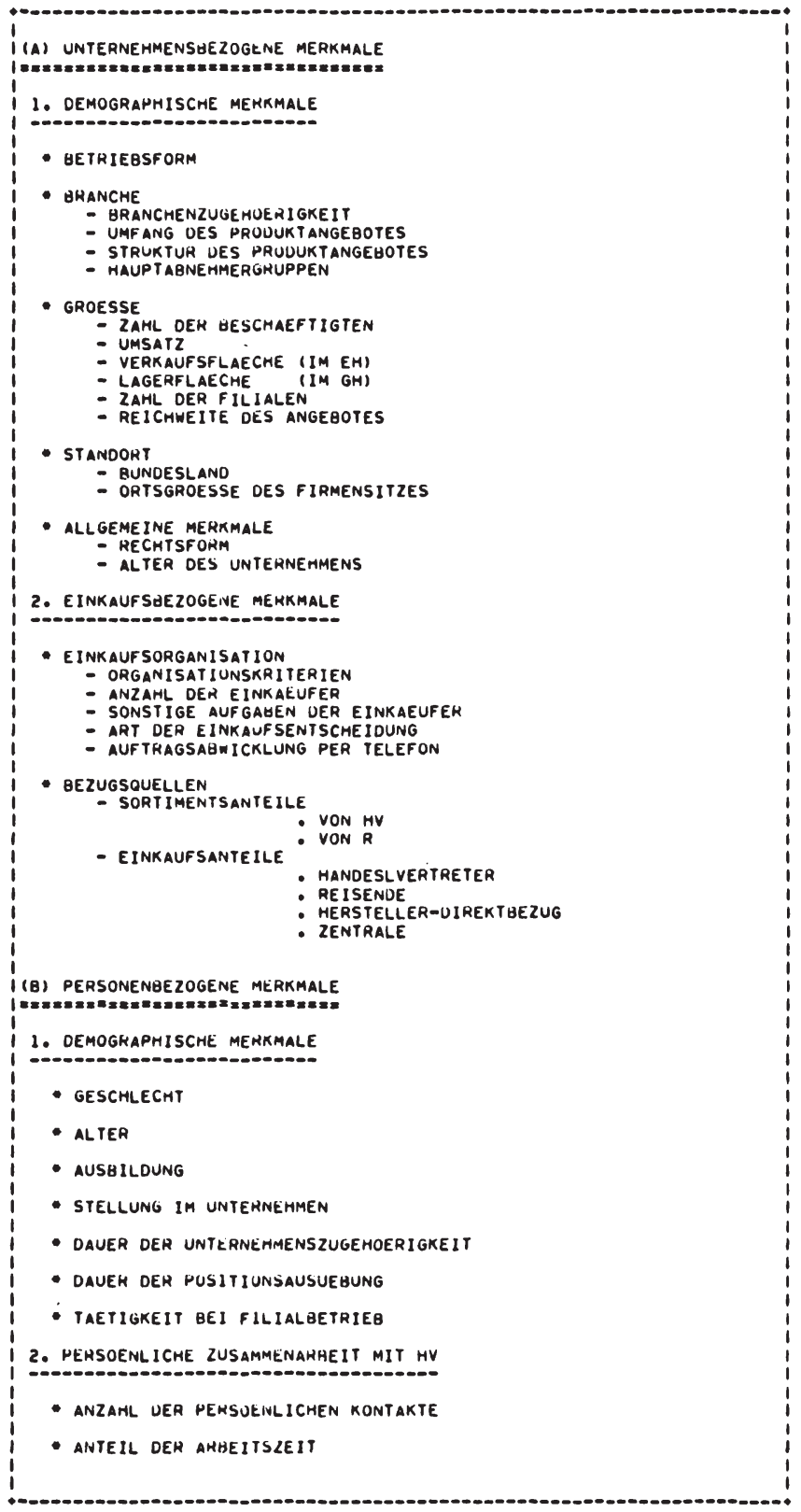

A88. B: GESTIMMUNGGSARTUREN VON RULLENERWARTUNG UNO -ERFUELLUNG BEIM KUNUENMARAT 


\subsection{Zusammenführung von Rollen und Bestimmungsfaktoren im Handelsvertreter-Marketing}

Mit der Diskussion handelsvertreterspezifischer Dienstleistungen als rollenprägenden Merkmalen und den Bestimmungsfaktoren dieser Leistungen auf allen Ebenen des Rollenmodells wurden die für eine Rollenanalyse im HandelsvertreterMarketing bedeutsamen abhängigen und unabhängigen Untersuchungsvariablen abgegrenzt. Der Einfluß einzelner Bestimmungsfaktoren auf die Rolle von Handelsvertretungen wurde dabei mehrfach exemplarisch deutlich.

Das Zusammenführen des Rollenmodells im HandelsvertreterMarketing (vgl. Abbildung 4) mit den Bestimmungsfaktoren einzelner Modellebenen mündet in ein umfassenderes Modell, das sich durch seine explizite Einbeziehung von Rollenkomponenten (ausgedrückt durch einzelne Dienstleistungen) und ihrem Determinantengerüst auszeichnet. Abbildung 9 veranschaulicht diese Grundstruktur einer Rollenanalyse im Handelsvertreter-Marketing. Die Darstellung ermöglicht es zugleich, die theoretische Analyse der Rolle von Handelsvertretungen im Absatzkanal sowie den Einfluß ihrer Bestimmungsfaktoren in Form von Hypothesen zusammenzufassen.

3. Hypothesen zur Rollenanalyse im Handelsvertreter-Marketing

Ausgehend von der dualen Stellung einer Handelsvertretung im Absatzkanal konnte unter Heranziehung rollentheoretischer Ubberlegungen ein verhaltenswissenschaftlicher Bezugsrahmen zur Analyse der Rolle einer Handelsvertretung im vertikalen Marketing aufgebaut werden. Die dabei vorgenommenen Konkretisierungen bzw. Operationalisierungen der Rollen- 


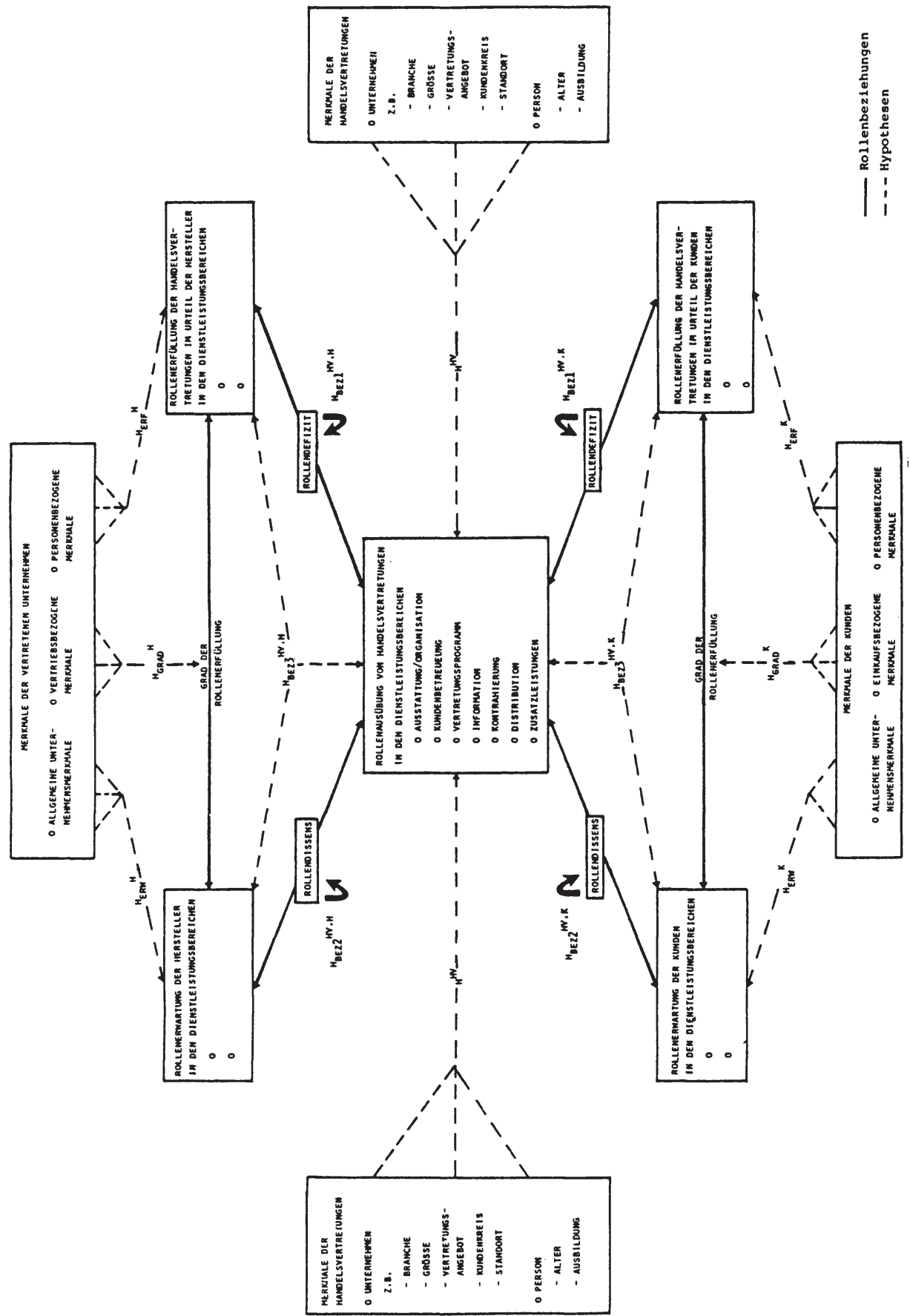


komponenten bedürfen zu ihrer Bestätigung einer empirischen Absicherung.

Als Zusammenfassung bisheriger zentraler Aussagen über die Rolle von Handelsvertretungen soll eine Reihe hypothetischer Annahmen formuliert werden ${ }^{1}$. Der HypothesenKatalog orientiert sich an dem vorgestellten Modell und dient in seiner strukturierung zugleich als Problemrahmen für die anschließende empirische Untersuchung. Die Einordnung der Hypothesen in das Modell wird durch eine gleichlautende Hypothesenkennzeichnung in Text und Abbildung unterstützt (vgl. Abbildung 9) ${ }^{2}$. Die formulierten Aussagen tragen vorwiegend den Charakter von Orientierungshypothesen, die je nach bisherigem Erkenntnisstand durch die Angabe möglicher Tendenzen verfeinert werden ${ }^{3}$.

\section{1 Hypothetische Annahmen einer rollenbezogenen Gruppie- rung der Dienstleistungsmerkmale}

Die Hypothesen einer rollenbezogenen Gruppierung der Dienstleistungsmerkmale von Handelsvertretungen formulieren Beziehungen für die Komponenten Rollenausübung, Rollenerfüllung und Rollenerwartung auf den drei Ebenen des Modells.

1 Zur Kennzeichnung absatzwirtschaftlicher Hypothesen vgl. Kopp, M., Hypothesenformulierung in der Absatzforschung, Betriebswirtschaftliche Schriften, Heft 60, Berlin 1972 .

2 Die Abbildung kann dabei nur die Hypothesen der zentralen Untersuchungsbereiche Rollenkomponenten und Rollenbeziehungen aufnehmen. Die übergeordneten Fragestellungen der Dienstleistungsstrukturierung (3.1) und Segmentierung der Modellebenen (3.4) lassen sich nicht unmittelbar veranschaulichen.

3 Zur Differenzierung von Orientierungs- und Tendenzhypothesen vgl. Bruhn, M., Das soziale Bewußtsein von Konsumenten, Wiesbaden 1978, S. 104 f.. Die formulierten Hypothesen drücken zugleich den erwarteten zusammenhang zwischen den betrachteten Variablen aus; auf die exaktere - aber inhaltlich gleichbedeutende - Angabe von Null- und Alternativhypothesen wird daher verzichtet. 


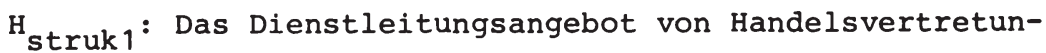
gen läßt sich auf wenige zentrale Bereiche verdichten. Dies gilt sowohl für die Rollenausübung der Handelsvertretungen, als auch für die Rollenerfüllung und -erwartung im Urteil der Marktpartner

(Hypothese der Rollengenerierung)

$\mathrm{H}_{\text {struk2 }}$ : Bei den ermittelten Rollenstrukturen des aktuellen Dienstleistungsangebotes auf den drei Befragungsebenen sind Hersteller- und Kundenbeurteilungen einander weitgehend ähnlich; das Selbstverständnis von Handelsvertretern führt im Vergleich $\mathrm{zu}$ einer abweichenden strukturierung der Rollenausübung

(Hypothese ungleicher Rollenstrukturen)

$\mathrm{H}_{\text {struk } 3}$ : Hersteller und Kunden richten im wesentlichen gleiche Erwartungen an das Dienstleistungsangebot von Handelsvertretungen (Hypothese homogener Rollenerwartungen)

$\mathrm{H}_{\text {struk 4 }}$ : Die Strukturierung der Dienstleistungsmerkmale nach der Rollenerwartung der Marktpartner weicht deutlich von der Gruppierung aktueller Leistungen $\mathrm{ab}$

(Hypothese ungleicher Rollenerfüllung und -erwartung).

\subsection{Hypothetische Annahmen zu einzelnen Rollenkomponenten}

Die Formulierung von Hypothesen zu den einzelnen Rollenelementen des vorgestellten Modells verkörpert eine erste zentrale Untersuchungseinheit im Rahmen einer Rollenanalyse 
des Handelsvertreter-Marketing. Ausgehend von den Komponenten der drei Modellebenen gilt es, die Verbindungen zu den Bestimmungsfaktoren der jeweiligen Ebene transparent zu machen.

1. Hypothesen zur Rollenausübung von Handelsvertretungen $\left(\mathrm{H}^{\mathrm{HV}}\right)$

$\mathrm{H}^{\mathrm{HV}}$ : Die Rollenausübung von Handelsvertretungen hängt $a b$ von unternehmens- und personenbezogenen Merkmalen der Handelsvertretung. Hinsichtlich des Erklärungswertes einzelner Bestimmungsfaktoren gilt:

$\mathrm{H}_{1}{ }^{\mathrm{HV}}$ : Unternehmensbezogene Faktoren sind eher geeignet, die Rollenausübung von Handelsvertretungen zu erklären als personenbezogene Einflußgrößen (Intergruppenhypothese der Rollenausübung)

$\mathrm{H}_{2}{ }^{\mathrm{HV}}$ : Innerhalb der unternehmensbezogenen Merkmale weisen abnehmerorientierte Faktoren (Vertretungsprogramm und Kundenkreis) höhere Erklärungsbeiträge auf als generelle Unternehmensmerkmale (Intragruppenhypothese der Rollenausübung)

$\mathrm{H}_{3}{ }^{\mathrm{HV}}$ : Der Erklärungswert unternehmensbezogener Merkmale kann durch die Einbeziehung personaler Faktoren verbessert werden. (Kombinationshypothese der Rollenausübung)

2. Hypothetische Annahmen $z u$ den Rollenkomponenten auf Herstellerebene $\left(\mathrm{H}^{\mathrm{H}}\right)$

Bei den Hypothesen auf der Ebene der vertretenen Unternehmen stehen Rollenerfüllung und Rollenerwartung - jeweils aus- 
gedrückt durch handelsvertreterspezifische Dienstleistungen - im Mittelpunkt der Hypothesenformulierung.

a. Hypothesen zur Rollenerfülling ( $\mathrm{Herf}^{\mathrm{H}}$ )

$\mathrm{H}_{\text {erf }}^{\mathrm{H}}$ : Die Beurteilung der Rollenerfüllung von Handelsvertretungen durch die Hersteller hängt ab von unternehmens- und personenbezogenen Merkmalen der vertretenen Unternehmen.

Hinsichtlich des Erklärungswertes der genannten Bestimmungsfaktoren gilt:

${ }^{\mathrm{H}}{ }_{\operatorname{erf1}}{ }^{\mathrm{H}}$ : Unternehmensbezogene Faktoren sind eher geeignet, die Beurtellung der Rollenerfüllung zu erklären als personale Einflußgrößen (Intergruppenhypothese der Rollenerfüllung)

${ }^{\mathrm{H}}{ }_{\text {erf2 }}{ }^{\mathrm{H}}$ : Innerhalb der unternehmensbezogenen Merkmale weisen die vertriebsorientierten Faktoren höhere Erklärungsbeiträge auf als generelle Unternehmmensmerkmale

(Intragruppenhypothese der Rollenerfüllung)

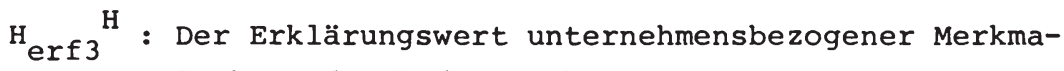
le kann durch die Einbeziehung personaler Faktoren verbessert werden

(Kombinationshypothese der Rollenerfüllung)

b. Hypothesen $z u$ den Rollenerwartungen ( ${ }_{\text {erw }}{ }^{\mathrm{H}}$ )

Die Hypothesen $z u$ den Rollenerwartungen der Hersteller werden analog zur Rollenerfüllung gebildet.

${ }_{\text {erw }}{ }^{\mathrm{H}}$ : Die Rollenerwartungen an Handelsvertretungen durch die Hersteller hängen ab von unternehmens- und 
personenbezogenen Merkmalen der vertretenen Unternehmen.

$\mathrm{H}_{\text {erw }}{ }^{\mathrm{H}}$ : Unternehmensbezogene Faktoren sind eher geeignet, die Rollenerwartungen zu erklären als personale Einflußgrößen

(Intergruppenhypothese der Rollenerwartung)

$\mathrm{H}_{\text {erw2 }}{ }^{\mathrm{H}}$ : Innerhalb der unternehmensbezogenen Merkmale weisen die vertriebsorientierten Faktoren höhere Erklärungsbeiträge auf als generelle Unternehmensmerkmale

(Intragruppenhypothese der Rollenerwartung)

$\mathrm{H}_{\text {erw3 }}{ }^{\mathrm{H}}$ : Der Erklärungswert unternehmensbezogener Merkmale kann durch die Einbeziehung personaler Faktoren verbessert werden

(Kombinationshypothese der Rollenerwartung)

3. Hypothetische Annahmen $z u$ den Rollenkomponenten auf Kundenebene $\left(\mathrm{H}^{\mathrm{K}}\right)$

Die Hypothesen auf der Ebene des Kundenmarktes folgen den Annahmen, die für die vertretenen Unternehmen von Handelsvertretungen vorgestellt wurden.

a. Hypothesen zur Rollenerfüllung ( $\mathrm{H}_{\text {erf }}{ }^{\mathrm{K}}$ ) $\mathrm{H}_{\text {erf }}^{\mathrm{K}}$ : Die Beurteilung der Rollenerfüllung von Handelsvertretungen durch die Kunden hängt $a b$ von unternehmens- und personenbezogenen Merkmalen der Kunden ${ }^{1}$.

1 Bei den Detailhypothesen sind die einkaufsbezogenen Merkmale an die Stelle der vertriebsorientierten Variablen zu setzen. 
$\mathrm{H}_{\text {erf1 }}{ }^{\mathrm{K}}$ : Intergruppenhypothese der Rollenerfüllung

$\mathrm{H}_{\text {erf2 }}{ }^{\mathrm{K}}$ : Intragruppenhypothese der Rollenerfüllung

$\mathrm{H}_{\text {erf }} 3^{\mathrm{K}}$ : Kombinationshypothese der Rollenerfüllung

b. Hypothesen $\mathrm{zu}$ den Rollenerwartungen $\left(\mathrm{H}_{\mathrm{erw}} \mathrm{K}\right.$ )

$\mathrm{H}_{\text {erw }}{ }^{\mathrm{K}}$ : Die Rollenerwartungen an Handelsvertretungen durch die Kunden hängen $a b$ von unternehmens- und personenbezogenen Merkmalen des Abnehmerkreises.

$\mathrm{H}_{\text {erw } 1}{ }^{\mathrm{K}}$ : Intergruppenhypothese der Rollenerwartung

$\mathrm{H}_{\text {erw2 }}{ }^{\mathrm{K}}$ : Intragruppenhypothese der Rollenerwartung

$\mathrm{H}_{\text {erw }} 3^{\mathrm{K}}$ : Kombinationshypothese der Rollenerwartung

\section{3 Hypothetische Annahmen zu Rollenbeziehungen}

Die Hypothesen über mögliche Beziehungen im Handelsvertreter-Marketing berücksichtigen sowohl horizontale als auch vertikale Rollenbeziehungen. Diese Hypothesengruppe stellt den zweiten zentralen Untersuchungsbereich der Rollenanalyse im Handelsvertreter-Marketing dar.

1. Hypothesen zum Grad der Rollenerfüllung auf Her-

$\mathrm{H}_{\text {grad1 }} \mathrm{H}$ : Der Grad der Rollenerfüllung ist als Abweichung zwischen der Beurteilung der Rollenerfüllung und den Rollenerwartungen der Hersteller meßbar (Hypothese differenzierter Rollenkomponenten) 
Die Hypothesen über den Einfluß der Bestimmungsfaktoren auf den Grad der Rollenerfüllung werden analog $\mathrm{H}_{\mathrm{erf}}$ und $\mathrm{H}_{\text {erw }}{ }^{\mathrm{H}}$ beibehalten. Diese Vorgehensweise impliziert eine weitgehende Invarianz der Determinanten in Bezug auf Rollenerfüllung und Rollenerwartung. Dies kommt auch in einem Vergleich der jeweiligen Hypothesen aus $\mathrm{H}_{\text {erf }}{ }^{\mathrm{H}}$ und ${ }_{\text {H }}{ }^{H}$ zum Ausdruck und bedeutet, daß die Rolle der Handelsvertretungen durch die Hersteller zwar unterschiedlich angesehen werden kann, diese unterschiedlichen Rolleneinschätzungen sich jedoch durch dieselben Merkmale bzw. Merkmalsgruppierungen erklären lassen.

Die detaillierten Hypothesen zum Grad der Rollenerfüllung werden deshalb nur in ihrer Kurzbezeichnung aufgeführt.

$\mathrm{H}_{\text {grad2 }}{ }^{\mathrm{H}}$ : Intergruppenhypothese zum Grad der Rollenerfüllung

$\mathrm{H}_{\text {grad3 }}{ }^{\mathrm{H}}$ : Intragruppenhypothese zum Grad der Rollenerfüllung

$\mathrm{H}_{\text {grad4 }}{ }^{\mathrm{H}}$ : Kombinationshypothese $\mathrm{z}$ um Grad der Rollenerfüllung.

B. Hypothesen zum Grad der Rollenerfüllung auf Kundenebene $\left(\mathrm{H}_{\operatorname{grad}}{ }^{\mathrm{K}}\right)$

Die Hypothesen für die Ebene der Abnehmer werden analog $z u$ den vertretenen Unternehmen formuliert:

$\mathrm{H}_{\text {grad1 }}{ }^{\mathrm{K}}$ : Hypothese differenzierter Rollenkomponenten $\mathrm{H}_{\text {grad2 }}{ }^{\mathrm{K}}$ : Intergruppenhypothese zum Grad der Rollenerfüllung

$\mathrm{H}_{\text {grad3 }}{ }^{\mathrm{K}}$ : Intragruppenhypothese zum Grad der Rollenerfüllung 
$\begin{aligned} \mathrm{H}_{\text {grad4 }} \mathrm{K} & \text { : Kombinationshypothese zum Grad der Rollener- } \\ & \text { füllung }\end{aligned}$

2. Hypothesen zu vertikalen Rollenbeziehungen

a. Hypothesen zu Rollenbeziehungen zwischen Handelsvertretungen und vertretenen Unternehmen ( $\mathrm{Hbez}^{\mathrm{HV} . \mathrm{H}}$ )

$\mathrm{H}_{\text {bez } 1} \mathrm{HV} \cdot \mathrm{H}$ : Rollenausübung aus der Sicht der Handelsvertretungen und Beurteilung ihrer Rollenerfüllung durch die Hersteller stimmen nicht überein; die Rollenerfüllung liegt dabei niedriger als die Rollenausübung

(Rollendefizithypothese)

$\mathrm{H}_{\text {bez2 }}{ }^{\mathrm{HV} \cdot \mathrm{H}}$ : Rollenerwartung der vertretenen Unternehmen und Rollenausübung der Handelsvertretungen stimmen nicht überein; die Erwartungen liegen tendenziell höher (Rollendissenshypothese)

Die Verknüpfung dieser beiden Hypothesen führt zu einer Aussage über die relative Stellung der Rollenkomponenten auf Hersteller- und Handelsvertretungsebene zueinander:

$\mathrm{H}_{\mathrm{bez} 3}{ }^{\mathrm{HV} \cdot \mathrm{H}}$ : Die Beurteilungen der Rollenkomponenten durch Handelsvertretungen und Hersteller führen $\mathrm{zu}$ einer Konstellation - d.h. relativen Stellung zueinander -, bei der die Rollenausübung zwischen Rollenerfüllung und Rollenerwartung liegt. Diese Konstellation zeichnet sich dadurch aus, daß Rollenausübung und -erfüllung einander ähnlicher sind als Rollenausübung und Rollenerwartung, d.h. das Rollendefizit ist geringer 
als der Rollendissens

(Hypothese der Rollenkonstellation)

b. Hypothesen $z u$ Rollenbeziehungen zwischen Handelsvertretungen und Kunden ( $\mathrm{H}_{\mathrm{bez}} \mathrm{HV}^{\mathrm{K}}$ )

Die Hypothesen über mögliche Rollenbeziehungen zwischen Handelsvertretungen und Kunden werden analog $\mathrm{zu}$ den Herstellern abgeleitet.

$\mathrm{H}_{\text {bez } 1}^{\mathrm{HV} \cdot \mathrm{K}}$ : Rollendefizithypothese

$\mathrm{H}_{\text {bez2 }}^{\mathrm{HV} \cdot \mathrm{K}}$ : Rollendissenshypothese

$\mathrm{H}_{\text {bez } 3}^{\mathrm{HV} \cdot \mathrm{K}}$ : Hypothese der Rollenkonstellation.

\subsection{Hypothetische Annahmen zur rollenbezogenen Segmentie- rung der Marktpartner}

Die vorgestellten zentralen Untersuchungsbereiche einer Rollenanalyse im Handelsvertreter-Marketing (Rollenkomponenten und -beziehungen) lassen die Notwendigkeit deutlich werden, auf allen Ebenen des beschriebenen Modells - d.h. bei allen Marktpartnern - Gruppen zu identifizieren, die sich auf der Basis rollenbezogener Merkmale im Hinblick auf die Ableitung handelsvertreterspezifischer Marketingstrategien als Zielgruppen eignen. Zur Identifikation und Beschreibung derartiger rollenbezogener Gruppierungen werden die folgenden Hypothesen überprïft. Die Darstellung beschränkt sich auf solche Rollenmerkmale, die für das Handelsvertreter-Marketing einen projektiven Charakter aufweisen. 
1. Hypothesen zur Segmentierung des Herstellermarktes $\left(\mathrm{H}_{\text {seg }}^{\mathrm{H}}{ }^{\mathrm{H}}\right.$

$\mathrm{H}_{\text {seg } 1}{ }^{\mathrm{H}}$ : Die Ebene der vertretenen Unternehmen läßt sich in Subgruppen zerlegen, die jeweils in sich weitgehend homogene Rollenerwartungen aufweisen; die Gruppen untereinander weisen deutlich heterogene Erwartungen auf ${ }^{1}$ (Hypothese der Segmentabgrenzung)

$\mathrm{H}_{\text {seg } 2}{ }^{\mathrm{H}}$ : Die gebildeten Gruppen homogener Rollenerwartungen lassen sich durch unternehmens- und personenbezogene Merkmale der vertretenen Unternehmen voneinander abgrenzen und damit für Marketingmaßnahmen nutzbar machen (Hypothese der Segmentbeschreibung)

2. Hypothesen zur Segmentierung des Kundenmarktes $\left(\mathrm{H}_{\text {seg }}{ }^{\mathrm{K}}\right.$ ) Die Hypothesen für den Kundenmarkt gelten analog: $\mathrm{H}_{\text {seg } 1}^{\mathrm{K}} \quad$ : Hypothese der Segmentabgrenzung $\mathrm{H}_{\text {seg } 2} \mathrm{~K}$ : Hypothese der Segmentbeschreibung

Die empirische Utberprüfung dieses Hypothesen-Katalogs war im Rahmen einer umfangreichen Untersuchung über die Stellung der Handelsvertretung im Absatzkanal möglich. Verfolgung der Hypothesen unter Veranschaulichung an konkreten Dienstleistungen von Handelsvertretungen ist Gegenstand der im folgenden Kapitel dargestellten empirischen Befunde.

1 Die hier vorgestellten Hypothesen sind deutlich von den weiter oben beschriebenen Annahmen einer rollenbezogenen Gruppierung der Dienstleistungsmerkmale zu trennen. Dort (vgl. B.3.1) ging es um die Strukturierung von Dienstleistungen; die hier vorgestellten Hypothesen betreffen dagegen Gruppierungen hinsichtlich des Ausmaßes möglicher Rollenerwartungen. 


\section{Empirische Untersuchung zum Marketing von Handels- vertretungen}

Die Überprüfung der Hypothesen zur Rolle von Handelsvertretungen im vertikalen Marketing war im Rahmen eines Forschungsprojektes des Instituts für Marketing der Universität Münster möglich. Nicht zuletzt wegen der mangelnden wissenschaftlichempirischen Forschung zum Spannungsfeld Hersteller-Handelsvertreter-Kunde ${ }^{1}$ wurde hier "eine Arbeit in Angriff genommen, um den Schwachstellen der bisherigen Forschung durch ein empirisch fundiertes Handelsvertreter-Marketing zu begegnen" 2 . Der Datensatz dieses Projektes bietet hinreichende Ansatzpunkte, die im Rahmen der vorliegenden Problemstellung formulierten Fragestellungen bzw. Hypothesen zu überprüfen.

Aus dem theoriegeleiteten Hypothesengerüst lassen sich die empirischen Untersuchungsbereiche unmittelbar ableiten. Der Diskussion inhaltlicher Problemstellungen wird im Verlauf des folgenden Kapitels eine Skizzierung von Konzeption und Durchführung der empirischen Studie sowie ihrer Stichprobenstruktur vorangestellt, um den Datenrahmen der Untersuchung transparent zu machen. Die anschließende Problemdiskussion folgt den vier Schwerpunktbereichen einer Rollenanalyse im Handelsvertreter-Marketing entsprechend dem vorgestellten $\mathrm{Hy}-$ pothesenkatalog. Nach einer rollenbezogenen Gruppierung der Dienstleistungsmerkmale von Handelsvertretungen gilt es, Ausprägungen und Bestimmungsfaktoren von Rollenkomponenten und -beziehungenim Handelsvertreter-Marketing für die drei Betrachtungsebenen (Hersteller, Handelsvertretung, Abnehmer) detailliert zu analysieren. Diese Diskussion differenzierter Einzelleistungen wird im letzten Schwerpunkt durch eine rollenbezogene Segmentierung der Marktpartner zusammengefaßt.

1 Die erste und bislang einzige Studie, die wesentliche Marktpartner von Handelsvertretungen einbezieht, haben Batzer und Meyerhöfer vorgelegt; vgl. Batzer, E., Meyerhöfer, W., a.a.O..

2 Meffert, H., Die Information ist verbesserungsfähig, in: HV + HM 1979, S. 1436 . 


\section{Untersuchungsdesign und Stichprobenstruktur}

Ausgehend von dem Grundgedanken, in ein empirisch fundiertes Handelsvertreter-Marketing die Vorstellungen und Meinungen aller beteiligten Marktpartner einzubeziehen, wurde im Rahmen des beschriebenen Forschungsprojektes ein umfassender Fragebogen zur Einschätzung sämtlicher Leistungsbereiche von Handelsvertretungen zusammengestellt. Entsprechend des Forschungsansatzes erfolgte die Erfassung der Beurteilung dieser Dienstleistungsbereiche mittels einer fünfstufigen Skala ${ }^{1}$.

Die Erhebung wurde als schriftliche Befragung mit einer positiven Ausschöpfungsquote ${ }^{2}$ von 24-42 8 in den drei Bereichen Handelsvertretungen, Hersteller (d.h. vertretene Unternehmen von Handelsvertretungen) und Abnehmer durchgeführt ${ }^{3}$. Die Befragungsteilnehmer wurden in allen Wirtschaftsbereichen, in denen Handelsvertreter tätig sind, durch eine $\mathrm{Zu}-$ fallsauswahl ermittelt ${ }^{4}$.

1 Zur Untersuchungsanlage des Forschungsprojektes vgl. Meffert, H., Kimmeskamp, G., Die Handelsvertretung im Meinungsbild von Industrie und Handel, a.a.O., S. 4 f. bzw. S. 90 .

2 Verzerrungen der Resultate aufgrund der non-response-Quote sind nicht zu erwarten, da nach umfangreichen persönlichen Testbefragungen sowie insbesondere einer Gegenüberstellung der Stichprobenstruktur mit verfügbaren Vergleichsdaten der vorliegende Rücklauf als repräsentativ angesehen werden kann. Vgl. hierzu insbes. Meffert, H., Kimmeskamp,G., Die Handelsvertretung im Meinungsbild von Industrie und Handel, a.a.0., s. 5 .

3 Vgl. ebenda, S. 5; die Eignung schriftlicher Erfassungsmethoden trotz relativ komplexer Fragebogen betonen Roscoe, A.M., Lang, D., Sheth, J.N., Follow-up Methods, Questionaire Length and Market Differences in Mail Surveys, in: JoM 1975, S. 20 ff.; Czaia, U., Schriftliche Befragung, in: IuA 1978, S. $174 \mathrm{ff.;}$ Hafermalz,0., Schriftliche Befragung-Möglichkeiten und Grenzen, Wiesbaden 1976, S. 159 ff..

4 Vgl. Bleymüller,J., Gehlert, G., Statistik für Wirtschaftswissenschaftler, in: Wist 1977,s. 579 f.; Berekoven, L., Eckert, W., Ellenrieder, P., Marktforschung. Methodische Grundlagen und praktische Anwendung, Wiesbaden 1977, S. $115 \mathrm{ff.:}$ Cohen, R. , Richmond,S., Stock,J.S., Semon, T. T. , Sampling in Marketing Research, AMA 'Marketing Research Techniques' Series No. 3, 0.0. 1970. 
Diese drei Befragungsgruppen erhielten jeweils segmentspezifisch angepaßte Fragebogen, wobei eine weitgehend gleiche Formulierung von Items zur Sicherstellung einer größtmöglichen Vergleichbarkeit sichergestellt war. Die in der vorliegenden Untersuchung verwendeten Fragestellungen dieser Studie sind in Anhang II beigefügt.

Der Befragungsumfang beträgt $n=237$ beim Kundensegment, $\mathrm{n}=402$ bei den Herstellern und $\mathrm{n}=419$ bei den Handelsvertretungen ${ }^{1}$. Abbildung 10 belegt zugleich die Streuung in den unterschiedlichen Wirtschaftsbereichen ${ }^{2}$. Der Kreis der vertretenen Unternehmen von Handelsvertretungen setzt sich vorwiegend aus produzierenden Betrieben zusammen, die teilweise gleichzeitig Waren importieren und zusammen mit eigenen Erzeugnissen über die Handelsvertreter absetzen. Der Kundenkreis umfaßt alle Betriebsformen des Handels und berücksichtigt ferner, daß Handelsvertreter zu einem nicht unerheblichen Teil Rohstoffe und Halbfabrikate an Industrieunternehmen verkaufen. Aus diesem Grund sind Industriebetriebe unabdingbarer Bestandteil des Kundensegmentes (vgl. Abbildung 11) ${ }^{3}$.

$1 \mathrm{Zu}$ den Erhebungsdaten vgl. Meffert, H., Kimmeskamp, G., a.a.O., S. $91 \mathrm{ff}$ und S. 98 .

2 Eine Gegenüberstellung dieser Stichprobenstruktur mit amtlichen Statistiken ist nicht möglich, da die letzte Handels- und Gaststättenzählung (HGZ) des statistischen Bundesamtes von 1968 die aktuelle Wirtschaftsstruktur nicht wiederzugeben vermag. Ein Vergleich mit der regelmäßig durchgeführten $\mathrm{CDH}-$ Statistik deckt weitgehende Ubereinstimmungen der Stichprobenstruktur auf. Ausgehend von Handelsvertretungen als zentralem Untersuchungsbereich kommt daher der Studie ein weitgehend repräsentativer Charakter zu. Vgl. Forschungsverband für den Handelsvertreter- und Handelsmaklerberuf (Hrsg.), CDH-Statistik 1978, Köln 1979,S. 29 (unveröffentlicht).

3 Keine Berücksichtigung finden in der studie die - zahlenmäBig - wenigen Handelsvertreter, die ausschließlich für Großhändler arbeiten sowie diejenigen, die vorwiegend an Endverbraucher (Konsumenten) verkaufen (z.B. Buchverlage, Zeitschriften). 


\begin{tabular}{|c|c|c|c|c|c|c|}
\hline \multirow{2}{*}{ WIRTSCHAFTSBEREICH } & \multicolumn{2}{|c|}{ HANDELSVERTRETER } & \multicolumn{2}{|c|}{ HERSTELLER } & \multicolumn{2}{|c|}{ KUNDEN } \\
\hline & $\begin{array}{l}\text { ANZAHL } \\
\text { FrAGEBOGEN }\end{array}$ & $\begin{array}{l}\text { ANTEIL } \\
(\%)\end{array}$ & $\begin{array}{l}\text { ANZAHL } \\
\text { FRAGEBOGEN }\end{array}$ & $\begin{array}{l}\text { ANTEIL } \\
(\%)\end{array}$ & $\begin{array}{l}\text { ANZAHL } \\
\text { FRAGEBOGEN }\end{array}$ & $\begin{array}{l}\text { ANTEIL } \\
(\%)\end{array}$ \\
\hline GRUNDSTOFFE & 46 & 11.0 & 64 & 15,9 & 26 & 11.0 \\
\hline INVESTITIONSGÜTER & 61 & 14.6 & 83 & 20.6 & 26 & 11,0 \\
\hline $\begin{array}{l}\text { INDUSTRIELLE GE- UND } \\
\text { VERBRAUCHSGUTER }\end{array}$ & 76 & 18,1 & 75 & 18.7. & 59 & 24,9 \\
\hline KONSUMGÜTER & 178 & 42,5 & 87 & 21.6 & 74 & 31.2 \\
\hline $\begin{array}{l}\text { NAHRUNGS- UND GENUSS- } \\
\text { MITTEL }\end{array}$ & 50 & 11.9 & 93 & 23.2 & 52 & 21,9 \\
\hline SONSTIGE & 8 & 1,9 & - & - & - & - \\
\hline SUMmE & 419 & 100,0 & 402 & 100,0 & 237 & 100.0 \\
\hline
\end{tabular}




\begin{tabular}{|l|c|}
\hline \multicolumn{1}{|c|}{ Betriebsform } & Anteil der Stichprobe \\
& in 8 \\
\hline Industriebetrieb & 29,2 \\
Einkaufsverband/Genossenschaft & 9,1 \\
Cash und Carry Markt & 4,1 \\
Fachgroßhandel & 32,9 \\
Discountbetrieb & 0,8 \\
Warenhaus / Filialbetrieb & 9,9 \\
Facheinzelhandel & 14,0 \\
\hline
\end{tabular}

Abb. 11: Struktur des Kundensegmentes nach Betriebsformen (Die Werte ergänzen sich zu mehr als $100 \%$, weil manche Kunden mehreren Betriebsformen angehören)

Auf der Basis dieses Datenmaterials ${ }^{1}$ werden im folgenden die zentralen Problemstellungen weiter analysiert. Dabei steht zunächst die Frage im Vordergrund, ob es gelingt, das Dienstleistungsangebot von Handelsvertretungen in geeigneter Form im Hinblick auf die theoretisch generierten Konstrukte $z u$ verdichten. Solchermaßen abgegrenzte - d.h. durch konkrete Dienstleistungen beschriebene - Ausprägungen von Rollenkonstrukten bilden die Voraussetzung für die anschließenden differenzierteren Auswertungen in den drei Befragungssegmenten.

$1 \mathrm{Da}$ an dieser Stelle nicht die Details aller einbezogenen Fragestellungen aufgezeigt werden können, informieren Tabellen im Anhang I ergänzend über weitere demographische Merkmale der drei Befragungsgruppen. Der Aufbau der Anhang-Tabellen 1, 3, 8 orientiert sich dabei an der aus Kapitel B. 2.2 bekannten Struktur der unabhängigen Untersuchungsvariablen. 
2. Ermittlung der zentralen Dienstleistungsbereiche von Handelsvertretungen

Die hypothetischen Annahmen einer rollenbezogenen Gruppierung der Dienstleistungsmerkmale von Handelsvertretungen weisen mehrere Ansatzpunkte auf, die diskutierten Rollenkonstrukte durch Gruppen von Dienstleistungsmerkmalen zu repräsentieren. Die dabei formulierte allgemeine Hypothese einer Rollengenerierung $\left(\mathrm{H}_{\text {struk } 1}\right)$ fordert in einem ersten Schritt die Analyse der Beurteilungen der Handelsvertretungen im Hinblick auf zentrale Bereiche ihrer Rollenausübung. Hersteller und Kunden haben ferner die Dienstleistungen in ihrem aktuellen Angebot sowie in ihrem Anspruchsniveau beurteilt (Rollenerfüllung bzw. -erwartung). In gleicher Weise wie bei den Handelsvertretungen sind daher in einem zweiten Schritt bei ihren Marktpartnern die entsprechenden Dienstleistungsstrukturen aufudecken.

Im Hinblick auf strategische tberlegungen bei der Entwicklung des Dienstleistungsangebotes ist es für Handelsvertretungen wichtig zu wissen, ob gegenwärtige Leistungserfüllung und zukunftsgerichtete Erwartungen der Marktpartner einander jeweils ähnlich sind, oder ob Rollenerfüllung und -erwartung bei beiden Marktpartnern jeweils unterschiedliche Dimensionen - d.h. unterschiedliche Dienstleistungsstrukturen - zugrundeliegen $\left(\mathrm{H}_{\text {struk } 4}\right)$. Weiterhin ergibt sich für eine Handelsvertretung aus ihrer Position im Spannungsfeld von Industrie und Handel die Notwendigkeit, Unterschiede in den Beurteilungen von vertretenen Unternehmen und Abnehmern aufzudecken $\left(\mathrm{H}_{\text {struk } 3}\right)$. Vor allem ein Vergleich mit dem eigenen Rollenverständnis bietet wertvolle Ansatzpunkte für den strategischen Rahmen zukünftiger Leistungen ( $\left.\mathrm{H}_{\text {struk } 2}\right)$. 
Zur Uberprüfung dieser Hypothesen wurden die Beurteilungen des Dienstleistungsangebotes von Handelsvertretungen auf allen drei Befragungsebenen faktorenanalytisch verdichtet ${ }^{1}$, um auf diese Weise die allgemeinen Konstrukte Rollenausübung, bzw. Rollenerfüllung und -erwartung problemspezifisch aufzuarbeiten und durch Bündel konkreter Dienstleistungen $z u$ veranschaulichen ${ }^{2}$.

\subsection{Komponenten der Rollenausübung von Handelsvertre- tungen}

Zur Ermittlung der zentralen Bereiche der Rollenausübung von Handelsvertretungen wurden die Beurteilungen aller in die Untersuchung einbezogenen Merkmale faktoriell verdichtet $^{3}$. Abbildung 12 zeigt die Gruppierung der Dienstleistungsmerkmale nach Durchlauf des Rechenverfahrens ${ }^{4}$.

1 Zur Anwendung der Faktorenanalyse vgl. Green, P.E., Analyzing Multivariate Data, Hinsdale 1978,s. 342 f.; Sturm,M.,Vajna,T., Grundzüge der Faktorenanalyse, in: Techniken der empirischen Sozialforschung, Hrsg.: J.V. Koolwijk, M. Wieken-Mayser, Bd. 5, Testen und Messen, München 1976, S. 184 ff.; Uberla, K., Faktorenanalyse, 2. Aufl., Berlin 1971.

2 Sämtliche Auswertungsarbeiten wurden mit dem SPSS-Programmpaket (Version 8) des Rechenzentrums der Universität Münster durchgeführt. Vgl. Nie, N.H., u.a., SPSS-Statistical Package for the Social Sciences, 2. Aufl., New York usw. 1975; Hull, C.M., Nie, N.H., SPSS Update, New York usw. 1979; Beutel, P., u.a., SPSS-Statistik Programmsystem für die Sozialwissenschaften, 2. Aufl., Stuttgart 1978.

3 zur Formulierung der entsprechenden Items vgl. Fragebogen 'Handelsvertreter' im Anhang II.

4 Die Abbildung gibt - wie auch die folgenden - aus Gründen einer besseren Ubersichtlichkeit und Verständlichkeit nur einen Ausschnitt der rotierten Faktormatrix wieder. Bei den einzelnen Faktoren sind jeweils die dominierenden Variablen aufgeführt (d.h. diejenigen mit den größten Faktorladungen). Die erklärten Varianzen basieren dagegen auf der vollständigen Faktorladungsmatrix, um hier den Informationsverlust zu minimieren. 


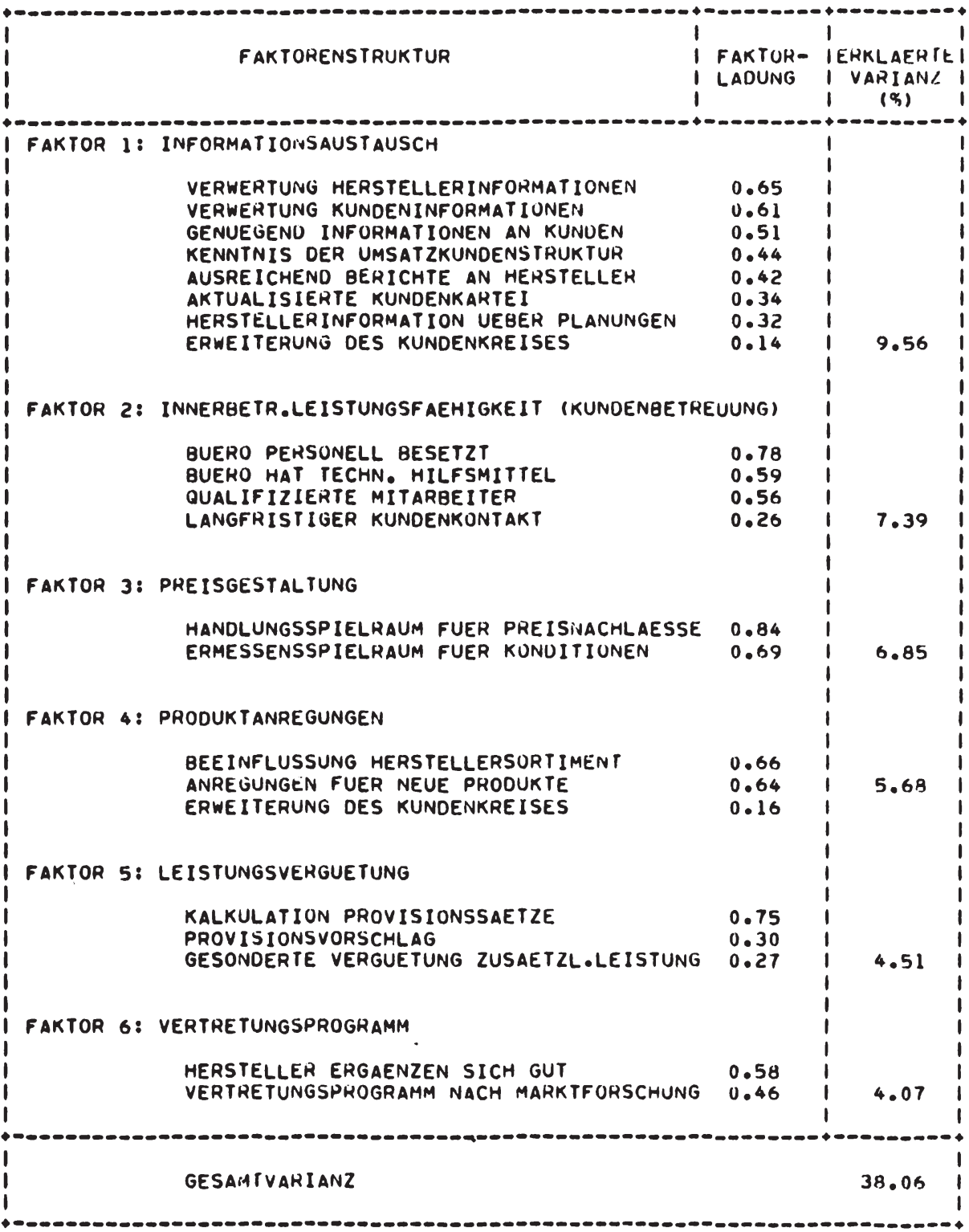

ABB. 12: STRUKTUH DER ROLLENAUSUEBUNG VON HAINUELSVERTRETUNGEN 
Aus den 21 in die Untersuchung einbezogenen Merkmalen wurden sechs Faktoren extrahiert, die insgesamt eine erklärte Varianz von 38 \& aufweisen. Dieser Erklärungsbeitrag entspricht dem methodischen Anspruch, zentrale Bereiche der Rollenausübung von Handelsvertretungen abzugrenzen ${ }^{1}$. Der relativ hohe Anteil merkmalsspezifischer Einzelrestvarianzen ist gleichzeitig Indikator für ein sehr differenziert ausgeprägtes Rollenbewußtsein der Handelsvertreter.

Betrachtet man die Gruppierung der Variablen in den einzelnen Bereichen der Rollenausübung, so lassen sich relativ gut voneinander abgrenzbare Bezeichnungen für die extrahierten Faktoren finden. Der Informationsaustausch mit beiden Marktpartnern sowie eine umfassende innerbetriebliche Leistungsfähigkeit zur Kundenbetreuung dominieren die Faktorenmatrix deutlich; auf diese beiden Gruppen entfällt nahezu die Hälfte des gesamten Erklärungsbeitrages. Dieses Resultat bestätigt vorherrschende Literaturmeinungen ${ }^{2}$ sowie frühere empirische Untersuchungen ${ }^{3}$.

Zwei weitere Merkmalsgruppen betreffen die Gestaltung des Leistungsangebotes selbst. Hier lassen sich zum einen die Produktanregungen gegenüber den vertretenen Unternehmen sowie die Gestaltung des Vertretungsprogramms der Handelsvertretung deutlich voneinander abgrenzen. Schließlich

1 Vgl. Zaltman, G., Burger, P.C., Marketing Research, Hinsdale 1975, S. 504. Der ermittelte Wert bewegt sich zudem auf einem empirisch vielfach ermittelten Niveau; vgl. ebenda sowie Stewart, D.W., The Application and Misapplication of Factor Analysis in Marketing Research, in: JoMR 1981 , S. 51 ff..

2 Vgl. Meffert, H., Die Handelsvertretung im Spannungsfeld des Marketing-Systems, a.a.0., S. 138; Batzer, E., Meyerhöfer, W., Der Handelsvertreter - individueller Absatzmittler im modernen Markt, a.a.O., S. 244; Gross, M., Dienstleistung - Chance des Handelsvertreters, in: HV + HM 1969 , S. 580 f.; Gutenberg, E., Grundlagen der Betriebswirtschaftslehre, a.a.O., S. $116 \mathrm{ff}$..

3 Vgl. Kimmeskamp, G., Tätigkeitsbereiche und Dienstleistungsangebot von Handelsvertretungen - Ergebnisse einer empirischen Untersuchung, a.a.O., S. 37 bzw. Abbildung 16. 
ist deutlich zwischen den Variablen der marktbezogenen preisgestaltung und der ausschließlich auf das Verhältnis Hersteller-Handelsvertreter ausgerichteten Leistungsvergütung zu unterscheiden.

Den auf der Basis der Handelsvertreterbeurteilungen gewonnenen Bereichen der Rollenausübung kommt ein lediglich explanativer Charakter zu. Im folgenden gilt es daher, die entsprechenden Gruppierungen auf seiten der vertretenen Unternehmen und Kunden vorzunehmen um die ermittelten Rollenstrukturen vergleichen zu können.

\subsection{Komponenten von Rollenerfüllung und-erwartung aus der Sicht der Marktpartner}

\subsection{Struktur der Rollenerfüllung und-erwartung im Urteil der vertretenen Unternehmen}

Dem Segment der vertretenen Unternehmen von Handelsvertretungen haben dieselben Dienstleistungsmerkmale zur Beurteilung vorgelegen wie den Handelsvertretungen ${ }^{1}$. Die Ergebnisse der faktoriellen Verdichtungen für die Einschätzung der gegenwärtigen Rollenerfüllung und für die Rollenerwartungen zeigen Abbildungen 13 und 14. Beide Analysen weisen einen ähnlichen Erklärungsbeitrag auf $(44,68$ für die Rollenerfüllung und 41 \& für die Rollenerwartung), wobei diese Erklärungsbeiträge geringfügig über der Gruppierung der Rollenausübung bei den Handelsvertretungen liegen $(38$ \%).

1 Zur Formulierung der Items vgl. Fragebogen 'Hersteller' im Anhang II. 


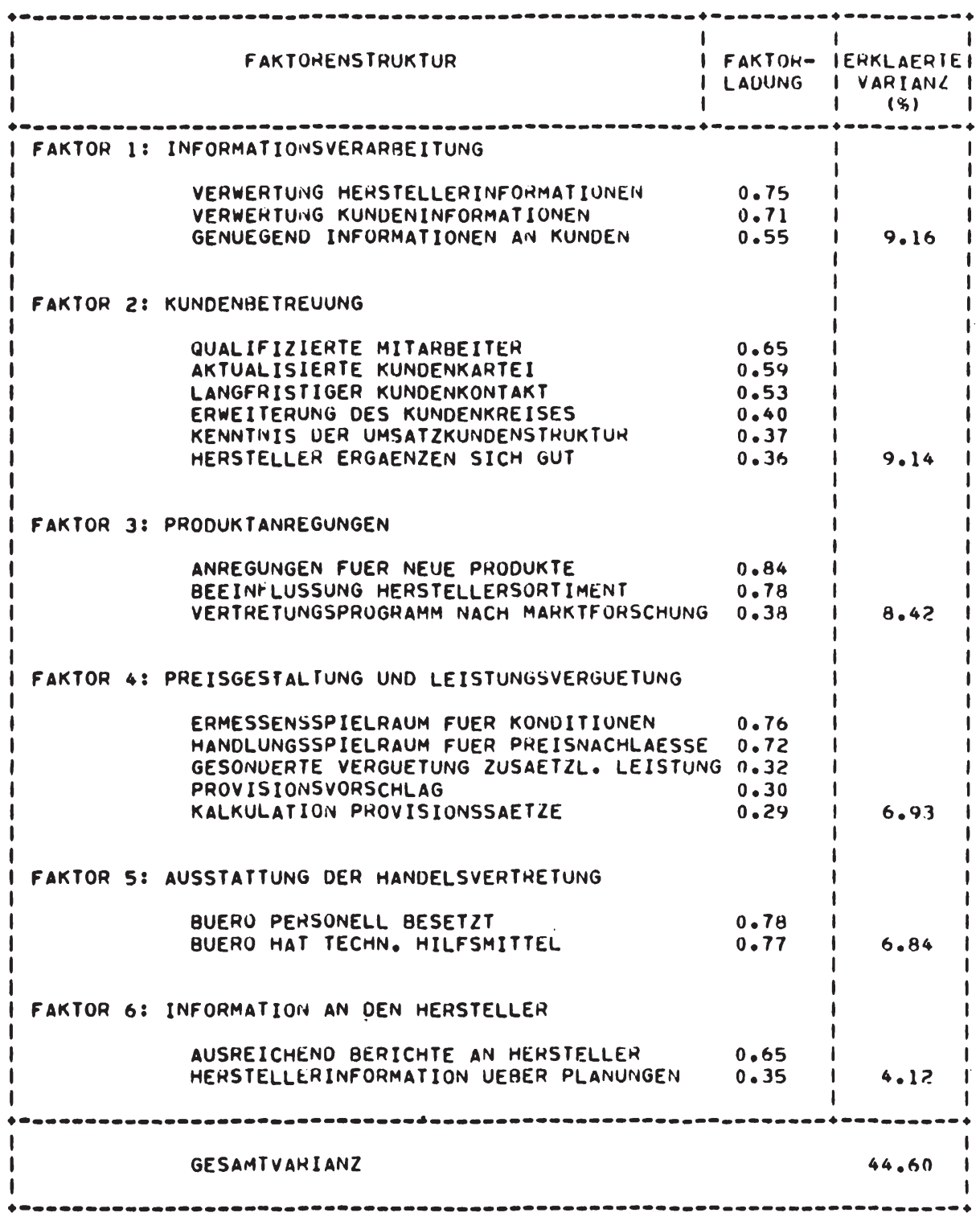

ABB. 13: STRUKTUR OER HOLLENERFUELLUNG VOIN HANOELSVEHTRETUNGEIN IM URTE IL DER VERTHETEHEN UNTERNEHMEN 


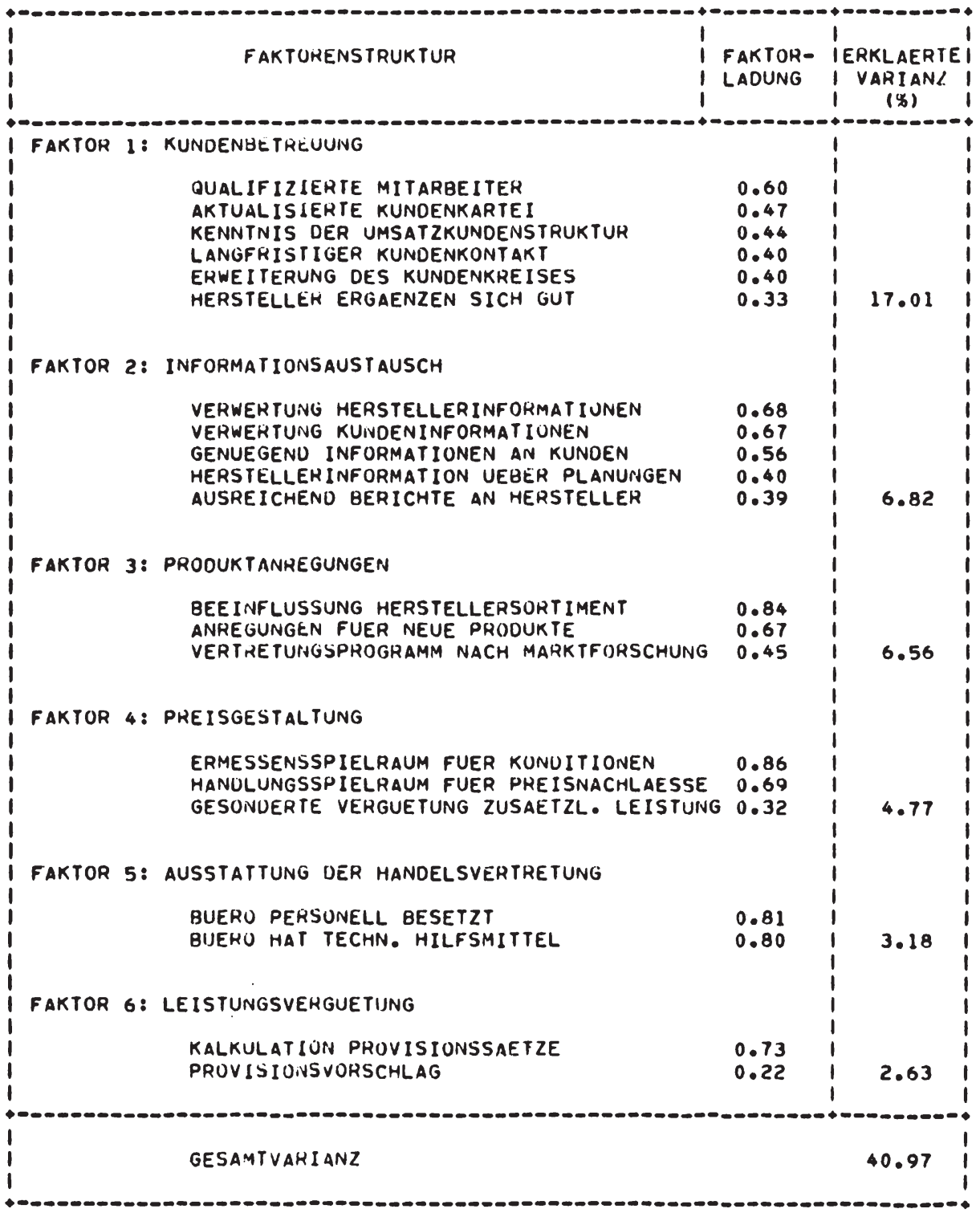

ABB. 14: STRUKTUR DER ROLLEINERWARTUNG AN HANDELSVERTRETUNGEN DURCH DIE VERTRËTENEN UNTERNEHMEN 
Erkennbare Unterschiede zeigen sich bei der Zusammensetzung der Faktoren von Rollenerfüllung und -erwartung in qualitativer und quantitativer Hinsicht. Bezüglich der quantitativen Erklärungsbeiträge ergeben sich bei der Rollenerfüllung sechs Faktoren mit Erklärungsbeiträgen zwischen 4 \& und 98 , bei der Rollenerwartung dominiert dagegen der Faktor Kundenbetreuung mit $17 \%$, die übrigen Faktoren liegen deutlich niedriger zwischen $3 \&$ und $7 \%$. Obwohl die Höhe des erklärten Varianzanteils allein keinen AufschluB über mögliche Aktivitätsniveaus geben kann, belegt die herausragende Stellung der Kundenbetreuung bei den Erwartungen der Hersteller dennoch die Notwendigkeit, diesem Dienstleistungsbereich besondere Aufmerksamkeit $\mathrm{zu}$ widmen. Bemerkenswert ist ferner, daß sich die in der Kundenbetreuung zusammengefaßten einzelnen Leistungsmerkmale zwischen der Beurteilung der Rollenerfüllung und der Rollenerwartung nicht verändern.

Verschiebungen bei der Gruppierung von Leistungsmerkmalen ergeben sich beim Informationsaustausch und den finanziell ausgerichteten Merkmalen. Während die Beurteilung der aktuellen Rollenerfüllung die Merkmale einer marktbezogenen Informationsverarbeitung (Abbildung 13 Faktor 1) von den herstellergerichteten Informationsaktivitäten trennt (Abbildung 13 Faktor 6), wird dieser Einschnitt bei den Rollenerwartungen der vertretenen Unternehmen aufgehoben (Abbildung 14 Faktor 2). Es wäre verfrüht, dieses Resultat als Rechtfertigung eines nur globalen und möglicherweise undifferenzierten Informationsverhaltens heranzuziehen. Vorliegende Resultate belegen vielmehr, daß gerade die Informationsaktivitäten der Handelsvertretungen verbesserungsbedürftig sind ${ }^{1}$.

1 Vgl. Meffert, H., Die Information ist verbesserungsfähig, a.a.O., S. 1442 . 
Ein umgekehrter Effekt zeigt sich bei den Bereichen Preisgestaltung und Leistungsvergütung. Während die Beurteilung der Rollenerfüllung hier keine Differenzierung ausweist (Abbildung 13, Faktor 4), belegen die Rollenerwartungen der vertretenen Unternehmen eine deutliche Trennung der Ansprüche in diesen Bereichen (Abbildung 14, Faktor 4 bzw. 6). Auch dieses Resultat läßt lediglich die Tendenzaussage zu, daß Handelsvertreter diese Bereiche gegenüber ihren vertretenen Unternehmen stärker trennen sollten, wobei das Ausmaß der Aktivitäten bei diesen Leistungen erst nach einer Gegenüberstellung der absoluten Ausprägungen von Rollenerfüllung und -erwartung festgelegt werden kann.

Die beiden übrigen Faktoren Produktanregungen und Ausstattung der Handelsvertretung bleiben in ihrer Zusammensetzung bei geringfügigen Verschiebungen der Faktorladungen erhalten (Faktoren 3 und 5 in Abbildungen 13 und 14).

Zusammenfassend läßt die verdichtende Analyse von Rollenerfüllung und Rollenerwartung auf seiten der vertretenen Unternehmen von Handelsvertretungen deutliche Parallelen im Aufbau der Rollenkonstrukte erkennen. Eine entsprechende Analyse des Kundenmarktes soll aufdecken, ob sich diese Tendenz bestätigt und welche Konsequenzen sich daraus für Handelsvertretungen ergeben.

\subsection{Struktur der Rollenerfüllung und -erwartung im Urteil der Kunden}

Der Befragungsgruppe 'Kunden' hat ein vom Umfang her reduzierter Katalog von Dienstleistungsmerkmalen zur Beurteilung vorgelegen. Dieses Vorgehen resultierte notwendigerweise aus der sachlichen Gegebenheit, daß einige Leistungs- 
merkmale lediglich das Verhältnis Handelsvertreter-Hersteller betreffen, die Kunden zu diesen Merkmalen aber keine Aussagen machen können (z.B. im Bereich der Provisionsabrechnung). Nach der Herausnahme solcher Merkmale verblieben elf Dienstleistungskomponenten, die für die Kunden von Handelsvertretungen der faktoriellen Verdichtung unterzogen werden konnten ${ }^{1}$.

Abbildungen 15 und 16 zeigen die ermittelten Dienstleistungsstrukturen aus der Beurteilung der Rollenerfüllung bzw. den Rollenerwartungen. Der Erklärungswert beider Analysen ist annähernd gleich hoch $(44,98$ bzw. 45,28) und bewegt sich damit in einer Höhe, die sich auch bei den beiden übrigen Befragungssegmenten herausgestellt hat. Damit gewinnt erneut die Hypothese Unterstützung, daß sich eine rollenbezogene Verdichtung des gesamten Dienstleistungsangebotes von Handelsvertretungen vornehmen läßt.

Die Resultate beim Kundensegment belegen eindrucksvoll, daß die Struktur der einzelnen Dienstleistungsbereiche sich bei Rollenerfüllung und -erwartung nicht verändert. Beide Konstrukte weisen die vier Gruppierungen Produktanregungen, Preisgestaltung, Kundenbetreuung und Vertretungsprogramm aus. Ähnlich zum Herstellersegment bestehen jedoch Unterschiede in der Gewichtung der Gruppierungen im Vergleich von Rollenerfüllung und -erwartung. Die Erklärungsbeiträge schwanken bei der Rollenerfüllung zwischen 7 is und 148 , bei der Rollenerwartung dominiert dagegen die Preisgestaltung mit einem Erklärungsbeitrag von 28 . Auch dieses Resultat kann erst nach einem Vergleich der absoluten Ausprägungen bei diesem Leistungsmerkmal in ein entsprechendes Aktivitätsniveau umgesetzt werden. Unbestreitbar ist jedoch die maßgebliche Rolle preispolitischer Aktivitäten von Handelsvertretungen in den Erwartungen des Kundenmarktes.

1 Zur Formulierung der Items vgl. Fragebogen 'Kunden' im Anhang II. 


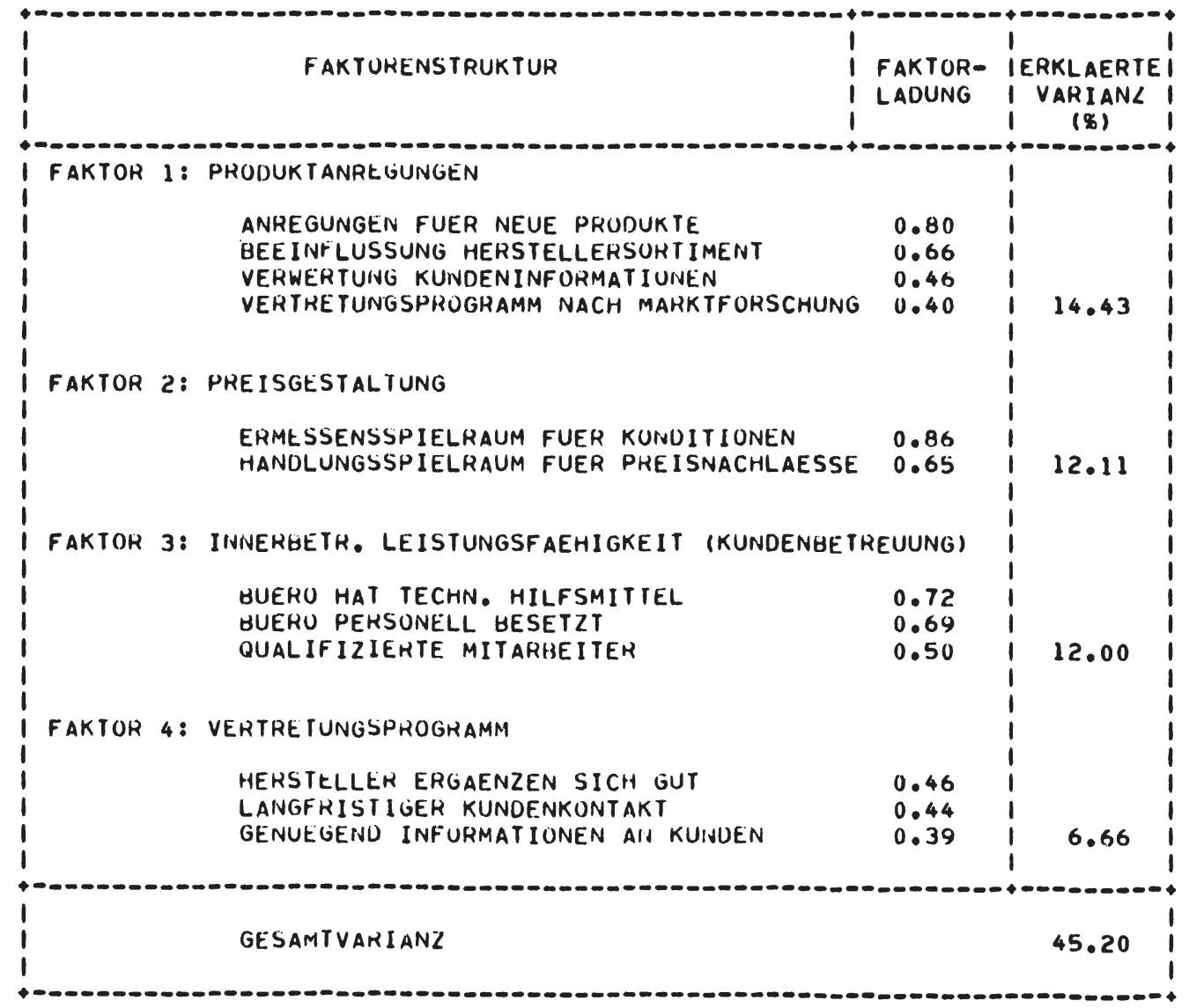

ABG. 15: STRUKTUR UER ROLLENERFUELLUNG VON HANDELSVERTRETUNGEN IM URTEIL OER KUNISE. IN 


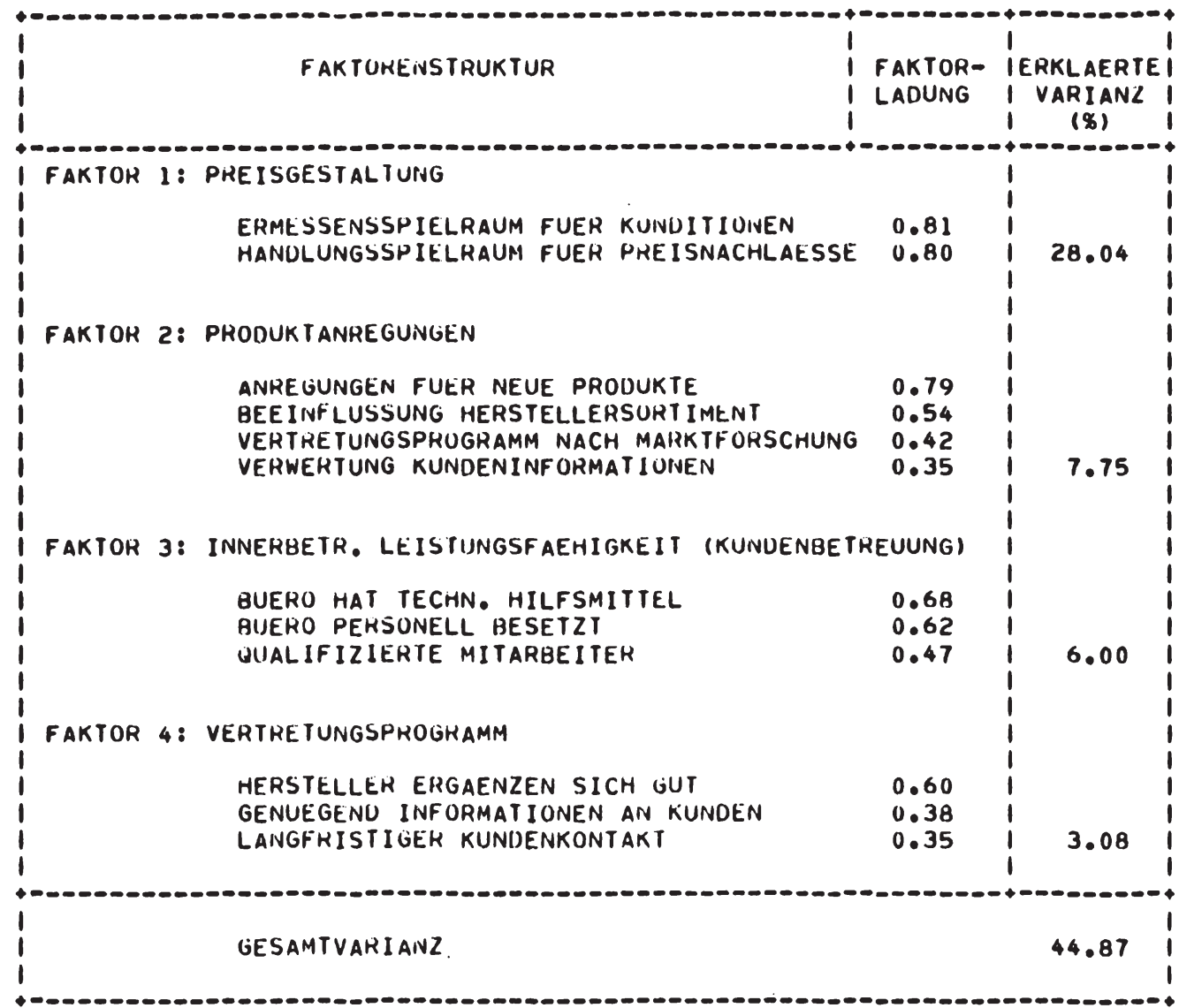

ABB. 16: STRUKTUR DER ROLLENERWARTUNG AN HANUELSVERTHETUNGEN DURCH DIE KUNUEN 
Die Gruppierung der Dienstleistungsmerkmale nach der Beurteilung der Rollenerfüllung sowie den Rollenerwartungen der Marktpartner stellt für Handelsvertretungen den ersten Schritt einer Neuorientierung ihres Dienstleistungsangebotes dar. In einem zweiten Schritt gilt es, die ermittelten Dienstleistungsstrukturen einander gegenüberzustellen, um auf diese Weise die Vorstellungen der Handelsvertreter selbst mit denen ihrer Marktpartner in Einklang zu bringen.

\subsection{Gegenüberstellung der ermittelten Dienstleistungs- strukturen}

Ein Vergleich der ermittelten Komponenten der Rollenausübung von Handelsvertretungen mit den strukturen der Rollenerfüllung und -erwartung auf seiten der Marktpartner vermag Unterschiede und Gemeinsamkeiten aller Beteiligten im Hinblick auf das Dienstleistungsangebot von Handelsvertretungen aufzudecken. Uber die Beschreibung dieser Sachverhalte hinaus verfolgt eine derartige vergleichende Analyse das Ziel, eine für alle Beteiligten gleichermaßen akzeptable Struktur des Dienstleistungsangebotes $z u$ ermitteln, die als Ausgangspunkt eines Handelsvertreter-Marketing angesehen werden kann. Damit soll im folgenden die Frage beantwortet werden, in welchem Maße die strukturen in der Beurteilung der Rollenerfüllung sowie der Rollenerwartungen der Marktpartner im Vergleich untereinander und in Bezug auf die Rollenausübung der Handelsvertretungen in Einklang stehen ( $\mathrm{H}_{\text {struk } 3,4}$ bzw. $\mathrm{H}_{\text {struk } 2}$ ).

Betrachtet man zunächst die Rollenstrukturen aus der Beurteilung von Herstellern und Kunden im Vergleich, deckt eine Gegenüberstellung der Komponenten der Rollenerfüllung eine weitgehende tbereinstimmung in der Gruppierung der 
Dienstleistungsmerkmale auf (Abbildung 13 und 15). Kundenbetreuung, Produktanregungen sowie preisgestaltung beinhalten nahezu identische Einzelmerkmale. Besonders auffällig ist dagegen, daß sich das Vertretungsprogramm lediglich bei den Kunden, herstellerbezogene Informationen nur bei den vertretenen Unternehmen als eigenständige Bereiche herauskristallisieren. An dieser stelle zeigen sich deutlich segmentspezifische Einflüsse, die es bei der Umsetzung im Rahmen von Marketingmaßnahmen durch Handelsvertretungen $z u$ beachten gilt.

Für die strategische Ausrichtung des HandelsvertreterMarketing gibt ein Vergleich der Rollenerwartungen von Herstellern und Kunden näheren Aufschluß. Ein Vergleich der hier ermittelten Dienstleistungsstrukturen unterstützt die Hypothese, daß die Rollenerwartungen an Handelsvertretungen von seiten ihrer Marktpartner weitgehend identisch sind. Segmentspezifische Abweichungen zeigen sich erneut beim Vertretungsprogramm (Kundensegment, Abbildung 16) und dem Informationsaustausch (Herstellersegment, $\mathrm{Ab}-$ bildung 14). Die Hypothese homogener Rollenerwartungen der Marktpartner von Handelsvertretungen kann nach diesen Resultaten hinsichtlich der Zusammensetzung des Dienstleistungsangebotes als bestätigt gelten.

Stellt man die Komponenten der Rollenausübung von Handelsvertretungen (Abbildung 12) den beschriebenen Strukturen der Rollenerfüllung und -erwartung der Marktpartner gegenüber, so kann die eingangs aufgestellte Hypothese ungleicher Rollenstrukturen zwischen Handelsvertretungen einerseits sowie Herstellern und Kunden andererseits nicht aufrecht erhalten werden. Die bei der Rollenausübung erkennbare strukturierung des Dienstleistungsangebotes deckt sich vielmehr in weiten Bereichen mit den Gruppierungen der Rollenerfüllung und -erwartung durch die Markt- 
partner. Die vorhandenen tbereinstimmungen zwischen Handelsvertretungen, vertretenen Unternehmen und Kunden formen damit eine hinreichende Basis für eine detaillierte Analyse der Ausprägungen und Bestimmungsfaktoren der diskutierten Rollenkonstrukte.

Greift man zusammenfassend die aufgestellten Hypothesen der rollenbezogenen Gruppierung der Dienstleistungsmerkmale von Handelsvertretungen auf, läßt sich folgendes feststellen:

Hstruk1 : Die Hypothese der Rollengenerierung hat sich als tragfähig erwiesen. Das Dienstleistungsangebot von Handelsvertretungen konnte sowohl in der Beurteilung der Rollenausübung als auch in der Einschätzung der Rollenerfüllung und -erwartung durch die Marktpartner auf wenige zentrale Bereiche verdichtet werden.

$\mathrm{H}_{\text {struk2 : Die Hypothese ungleicher Rollenstrukturen }}$ zwischen Handelsvertretungen und ihren Marktpartnern kann nicht aufrecht erhalten werden. Die festgestellte Ubereinstimmung hinsichtlich der strukturierung des Dienstleistungsangebotes spannt somit den strategischen Rahmen für das Handelsvertreter-Marketing auf, d.h. die generierten Gruppierungen bilden den Ausgangspunkt der Planung von Marketingaktivitäten.

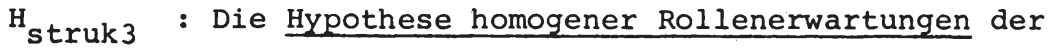
Marktpartner hat sich bestätigt. Hier gilt es - wie auch bei den anderen Hypothesen - zu beachten, daß diese aufgedeckten Ubereinstimmun- 
gen sich auf die Struktur des Leistungsangebotes beziehen, nicht aber auf das Ausmaß der jeweiligen Aktivitäten. Die hier aufgedeckten strukturellen Ubereinstimmungen vereinfachen somit den Prozeß der Planung konkreter Maßnahmen.

$\mathrm{H}_{\text {struk } 4}$ : Ein Vergleich von Rollenerfüllung und -erwartung im Urteil der Marktpartner deutet in dieselbe Richtung. Hier erweist sich die Hypothese ungleicher Strukturen von Rollenerfüllung und-erwartung als ambivalent. Während sich die qualitative Zusammensetzung der Dienstleistungsgruppierungen nicht erheblich unterscheidet, müssen die quantitativen Unterschiede in den Erklärungsbeiträgen als Indikator für eine sorgfältige Analyse der Ausprägungen und Bestimmungsfaktoren der Beurteilungen aller drei Beteiligtengruppen verstanden werden. 
3. Analyse der Rollenkomponenten im HandelsvertreterMarketing

Die Analyse der Rollenkomponenten (d.h. Rollenausübung, -erfüllung und -erwartung) untergliedert sich entsprechend dem vorgestellten Modell in drei Ebenen. Als Ausgangspunkt der Betrachtung wird die Rollenausübung von Handelsvertretungen als zentralem Modellelement vorgestellt. Ergebnisse und Interpretationen $\mathrm{zu}$ Rollenerfüllung und -erwartung jeweils für Hersteller- und Kundenebene schließen sich an.

Zur Strukturierung der Diskussion dienen die im vorhergehenden Kapitel erarbeiteten empirischen Dienstleistungsstrukturen auf allen drei Ebenen als Auswertungsgerüst, d.h. $z u$ jedem der aufgezeigten Dienstleistungsbündel werden die entsprechenden Bestimmungsfaktoren herausgearbeitet. Die Darstellung ist dabei einheitlich so konzipiert, daß differenzierte Aussagen sowohl über die Erklärungswirksamkeit der unabhängigen Variablen als auch über einzelne Leistungsbereiche getroffen werden können.

\subsection{Rollenausübung von Handelsvertretungen}

Die theoriegeleiteten Hypothesen zur Rollenausübung von Handelsvertretungen lenken das Untersuchungsinteresse unmittelbar auf eine Verknüpfung zwischen abhängigen Variablen (hier: Dienstleistungsmerkmalen) und unabhängigen Variablen (hier: unternehmens- und personenspezifischen Merkmalen). Eine solchermaßen integrative Analyse von Variablengruppen setzt jedoch eine differenzierte Detailkenntnis der Ausprägungen abhängiger und unabhängiger Merkmale voraus. Da eine solche ausführliche Präsentation durch ihren vorwiegend dokumentarischen Charakter nur eine geringe Nähe 
zum vorliegenden Problem aufweist, wird auf eine Diskussion der Variablenausprägungen verzichtet. Gleichwohl bietet die Kenntnis dieser Ausprägungen wertvolle Hilfen zur Argumentation und Veranschaulichung, wenn es im folgenden darum geht, die Bestimmungsfaktoren der Rollenausübung von Handelsvertretungen herauszuarbeiten. Aus diesem Grunde ist die Deskription der Rollenausübung sowie sämtlicher unabhängiger Variablen im Anhang I zusammengefaßt ${ }^{1}$.

\section{11 Bestimmungsfaktoren der Rollenausübung im Uberblick}

Die aus dem theoriegestützten Modell abgeleiteten Hypothesen zur Rollenausübung von Handelsvertretungen erfordern zu ihrer Uberprüfung die Auswahl geeigneter Rechenverfahren. Für die im vorliegenden Fall angestrebte Erklärung der Ausübung von Dienstleistungen durch eine Reihe von unternehmens- und personendemographischen Merkmalen wurde das Verfahren der Regressionsanalyse herangezogen.

Aufgabe dieses Verfahrens ist es, die Art der Abhängigkeit zwischen quantitativen - d.h. metrisch skalierten - Variablen $z u$ bestimmen ${ }^{2}$. Bei empirisch-sozialwissenschaftlichen Fragestellungen hat sich dabei die Annahme linearer Wirkungszusammenhänge vielfach bewährt ${ }^{3}$. Da vorliegende

1 Vgl. Tab. 1 und 2, Anhang $I$.

2 Vgl. Bleymüller, J., Gülicher, H., Gehlert, G., Statistik für Wirtschaftswissenschaftler, in: Wist 1978, S. 369 ff.; Hüttner, M., Informationen für Marketing-Entscheidungen, München 1979, S. $238 \mathrm{ff.;}$ Yamane, T., Statistik, Frankfurt 1976, S. $331 \mathrm{ff.;}$ Green, P.E., a.a.O., S. $50 \mathrm{ff..}$

3 Vgl. Bleymüller, J., Gülicher, H., Gehlert, G., a.a.O., S. 370; Yamane, T., a.a.O., S. 385 f.. 
Hypothesen nur Aussagen über Gruppen von unabhängigen Merkmalen treffen konnten, muß mit Hilfe der Regressionsanalyse ein Weg gefunden werden, die Erklärungswirksamkeit einzelner Variablen aus diesen Gruppen zu ermitteln, um konkretere Aussagen im Hinblick auf maßnahmenspezifische Empfehlungen ableiten zu können. Diese Notwendigkeit wird durch die Verwendung der schrittweisen multiplen Regression weitgehend erfüllt ${ }^{1}$. Bei diesem Verfahren ist es möglich, durch sukzessive Einbeziehung der unabhängigen Variablen ${ }^{2}$ ihren jeweiligen Erklärungszuwachs für die abhängige Variable unter Anwendung bestimmter Ausschlußkriterien zu ermitteln. Für die vorliegenden Datenauswertungen wurde ein Signifikanzniveau von 95 \& für eine einzubeziehende Variable als Trennkriterium verwendet ${ }^{3}$.

Für die zu erklärenden, d.h. die abhängigen Variablen der Analyse wurden die faktoriell verdichteten Dienstleistungsbündel herangezogen, wobei jeder der beschriebenen Faktoren jeweils eine abhängige Variable bildet ${ }^{4}$. Durch die Verwendung der entsprechenden Faktorenwerte war es so mög-

1 Vgl. Gülicher, H., Gehlert, G., Statistik für Wirtschaftswissenschaftler, a.a.O., S. $532 \mathrm{ff}$; Hüttner, M., a.a.O., S. 247 f.; Kim, J.O., Kohout, F.J., Multiple Regression Analysis: Subprogramm Regression, in: SPSS, a.a.O., S. $320 \mathrm{ff}$..

2 Die vorgestellten unternehmens- und personendemographischen Merkmale der Handelsvertretungen weisen nicht alle metrisches Skalenniveau auf. Solche Merkmale (z.B. Gebietsstruktur) lassen sich über eine Umformung in Dummy-Variable für eine Regressionsanalyse aufbereiten. Vgl. hierzu Hüttner, M., a.a.O., S. 261 f.; Kim, J.O., Kohout, F.J., Special Topics in Linear Models, in: SPSS, a.a.O., S. 373 ff.; Gülicher, H., Gehlert, G., a.a.O., S. $579 \mathrm{f}$..

3 Das SPSS-Programm Regression stellt zur Ermittlung dieser Signifikanzgrenze die Ergebnisse eines F-Tests bereit; vgl. hierzu Hüttner, M., a.a.O., S. 251 f.; Kim, J.O., Kohout, F.J., a.a.O., S. 334 ff.; Gülicher, H., Gehlert, G. , a.a.O., S. 575.

4 Dazu wurden Faktorenwerte (facscores) für die Regressionsrechnungen als abhängige Variablen verwendet. 
lich, das gesamte Dienstleistungsprogramm auf eine überschaubare Anzahl von Merkmalen zu beschränken. Gleichzeitig stellt die Vorgehensweise durch die Einbeziehung des gesamten umfassenden Dienstleistungskatalogs in den Faktoren sicher, daß Informationsverluste durch eine andersartige Beschränkung bei der Variablenauswahl vermieden werden $^{1}$.

Die Regressionen zwischen den beschriebenen Dienstleistungsbündeln und den verschiedenen Gruppen unabhängiger Merkmale zeigt Abbildung 17 in einem Ergebnisüberblick ${ }^{2}$. Es wird deutlich, daß die unternehmensbezogenen Merkmalsgruppen und auch die personenbezogenen Variablen jeweils isoliert nur relativ geringe Beiträge zur Erklärung der Rollenausübung in den Dienstleistungsbereichen liefern. (Spalten 1, 2, 3 und 5). Die simultane Berücksichtigung mehrerer Merkmalsgruppen führt dagegen $z u$ erheblich höheren Erklärungsbeiträgen (Spalten 4 und 6), wobei die Einbeziehung personaler Merkmale den Erklärungswert unternehmensbezogener Faktoren teilweise deutlich $\mathrm{zu}$ erhöhen vermag.

Damit gilt es, als erstes wesentliches Resultat festzustellen, daß die Rollenausübung von Handelsvertretungen in allen Dienstleistungsbereichen durch eine Reihe unterschiedlicher Faktoren beeinflußt wird. Es läßt sich keine Merkmalsgruppe abgrenzen, die einen dominanten Einfluß

1 Dieser Informationsverlust ist nicht $\mathrm{zu}$ verwechseln mit der Diskrepanz zwischen der erklärten Varianz aller Faktoren und der erklärbaren Gesamtvarianz. Diese resultierende Einzelrestvarianz kann mathematisch nicht wie etwa die Faktorenwerte - weiterverarbeitet werden und muß bei Interpretationen ergänzend herangezogen werden.

2 Die unternehmens- und personenbezogenen Merkmale der Handelsvertretungen beziehen sich dabei auf die Diskussion dieser Bestimmungsfaktoren in Kap. B. 2.21. Die in der Abbildung vorgestellten Resultate fassen die Ergebnisse der jeweiligen multiplen Regressionsanalysen zusammen. 


\begin{tabular}{|c|c|c|c|c|c|c|}
\hline $\begin{array}{l}\text { Merkmale der } \\
\text { Handelsvertretung }\end{array}$ & $\begin{array}{l}\text { Grösse } \\
\text { (1) }\end{array}$ & $\begin{array}{l}\text { Vertre- } \\
\text { tungsange- } \\
\text { bot } \\
\quad \text { (2) }\end{array}$ & $\begin{array}{l}\text { Kunden- } \\
\text { kreis } \\
\text { (3) }\end{array}$ & $\begin{array}{l}\text { Untermeh- } \\
\text { mensmerkma- } \\
\text { le gesamt } \\
(1+2+3) \\
(4)\end{array}$ & $\begin{array}{l}\text { personale } \\
\text { Merkmale } \\
\text { (5) }\end{array}$ & $\begin{array}{l}\text { Unternehmens- } \\
\text { und personen- } \\
\text { bezogene Merkmale } \\
\qquad(4+5) \\
(6)\end{array}$ \\
\hline $\begin{array}{l}\text { Dienstleistungs- } \\
\text { bereich }\end{array}$ & $n_{\mathrm{v}} 1$ & $n_{v} \mid r$ & \begin{tabular}{l|l}
$n_{v}$ & 1 \\
& $r$
\end{tabular} & $n_{v 1} \quad r$ & $n_{v}{ }_{1}^{1}$ & $\begin{array}{ll}n_{v} & 1 \\
& 1\end{array}$ \\
\hline Informationsaustausch & $\left.2\right|_{1} ^{1} 0,2+$ & $\begin{array}{r}1 \\
-1-\end{array}$ & $\begin{array}{c}-1- \\
1\end{array}$ & $61_{0,27}^{1}$ & $-1-$ & \begin{tabular}{l|l}
1 \\
7
\end{tabular} \\
\hline $\begin{array}{l}\text { innerbetriebliche } \\
\text { Leistungsfähigkeit }\end{array}$ & $3_{1}^{1} 0,58^{+}$ & $2 \mid 0,39^{+}$ & $310,4 \pi$ & 10. ${ }_{1}^{1} 0,65^{+}$ & ${ }_{4}^{1} 0,3 \frac{1+}{1+}$ & $13^{1} 0,67^{+}$ \\
\hline Preisgestaltung & $2 \quad 10,14$ & $-1-$ & $-1-$ & $410,22^{+}$ & $\left.1\right|^{0,16^{+}}$ & $11,0,3 \hat{3}^{+}$ \\
\hline Produktanregungen & $-1-$ & $11^{0,1 \frac{1}{2}}$ & 4. $1^{0,2^{3+}}$ & $9_{1}^{1} 0,4 b^{+}$ & $-1-$ & $9 \mid 0,4 t^{+}$ \\
\hline Leistungsvergütung & $\begin{array}{r}-1- \\
1\end{array}$ & $-\frac{1}{1}-$ & $-1-$ & $91^{0,29^{+}}$ & $1^{1} 0,1 \frac{1}{2}$ & $\left.7\right|_{0,28} ^{+}$ \\
\hline Vertretungsprogramm & $\begin{array}{l}10,1 t^{+} \\
\end{array}$ & $2,0,17$ & $\begin{array}{r}-1- \\
1\end{array}$ & $\begin{array}{ll}8 & 10,29^{+} \\
1 & 1\end{array}$ & $4 \quad 0,18$ & $\left.10\right|_{0,33^{+}}$ \\
\hline
\end{tabular}

Abb. 17: Bestimmungsfaktoren der Rollenausübung von Handelsvertretungen im Uberblick

$n_{v}:$ Anzahl der in die multiplen Regressionen einbezogenen Variablen

$r$ : multipler Korrelationskoeffizient

+ : 99 \& - Signifikanzniveau

$++: 95$ - Signifikanzniveau 
aufweist. Dieses relativ globale Urteil läßt sich durch eine differenzierte Analyse einzelner Leistungsbereiche weiter verfeinern. Im folgenden werden daher die Bestimmungsfaktoren einzelner Dienstleistungsbereiche separat herausgearbeitet.

\subsection{Bestimmungsfaktoren der Rollenausübung einzelner Dienstleistungsbereiche}

\subsection{Informationsaustausch}

Betrachtet man den Leistungsbereich Informationsaustausch in Abbildung 17, so fällt auf, daß lediglich Faktoren, die die Größe einer Handelsvertretung beschreiben, einen Einfluß auf die Rollenausübung in diesem zentralen Leistungsbereich von Handelsvertretungen haben. Wider Erwarten treten Merkmale, von denen ein Einfluß zu erwarten gewesen wäre - wie z.B. die Komplexität technischer Produkte - nicht in Erscheinung. Auch führt der unmittelbare interpersonelle Charakter der Informationsaktivitäten nicht zu Erklärungsbeiträgen für die personenbezogenen Merkmale eines Handelsvertreters.

Abbildung 18 dokumentiert, welche Faktoren sich für den Informationsaustausch als erklärungswirksam herausgestellt haben. 


\begin{tabular}{|l|c|c|}
\hline \multicolumn{1}{|c|}{ ERKLÄRENDE VARIABLE } & $\begin{array}{c}\text { MULTIPLE } \\
\text { KORRELATION } \\
(\mathrm{r})\end{array}$ & $\begin{array}{c}\text { BEEINFLUSSUNGS- } \\
\text { RICHTUNG }\end{array}$ \\
\hline VERMITTELTER WARENUMSATZ & 0.19 & - \\
STUDIUM & 0.22 & + \\
BRUTTOEI NNAHMEN & 0.24 & - \\
ZAHL DER KUNDEN & 0.26 & + \\
MEHRERE WARENGRUPPEN & 0.27 & + \\
GRUNDSTOFFE & 0.28 & - \\
HAUPTVERTRETUNG VORHANDEN & 0.29 & + \\
\hline
\end{tabular}

ABB, 18: UNTERNEHMENS- UND PERSONENBEZOGENE BESTIMMUNGSFAKTOREN DER ROLLENAUSUBBUNG BEIM INFORMATIONSAUSTAUSCH

Nach dem vorliegenden Resultat werden Informationsaktivitäten in erster Linie von großen Handelsvertretungen (hohe Umsätze, Bruttoeinnahmen) mit einem umfangreichen Kundenkreis als wichtiger Leistungsbestandteil angesehen. Kleinere Handelsvertretungen neigen demnach eher dazu, diesen Bereich ihrer Tätigkeit zu vernachlässigen; diese Vermutung erfährt Unterstützung durch das Resultat, daß vorwiegend Handelsvertreter mit Studienabschluß den Informationsaktivitäten eine hohe Priorität einräumen ${ }^{1}$.

1 Gerade Aktivitäten im Informationsbereich werden von Herstellern und Handel als besonders wichtig eingestuft. Vgl. Koinecke, J., Marketing mit Handelsvertretern im härteren Markt, a.a.O., S. 196; ein Informationsdefizit des Handels bei Markt- und Brancheninformationen belegt die Studie: Der Markenartikel-Reisende, Hrsg.: Lebensmittel-zeitung, 0.0., 1978; O.V., Verkaufsleiter: Resultat einer Umfrage, in: VuM, Heft 3, 1978, S. 44 f.. 


\subsection{Innerbetriebliche Leistungsfähigkeit}

Die Einschätzung der innerbetrieblichen Leistungsfähigkeit läßt sich durch eine Reihe demographischer Merkmale der Handelsvertretungen erklären. Bereits die zusammengefaßten Resultate (Abbildung 17) machten deutlich, daß beispielsweise Erreichbarkeit und Mitarbeiterqualifikation in hohem Maße von kunden-, größen- und personenbezogenen Faktoren determiniert werden.

Bei den kundenbezogenen Merkmalen weisen Art der Auftragsabwicklung, Auftragsumfang und $\mathrm{Zahl}$ der Kunden darauf hin, daß mit einem Ansteigen der genannten Faktoren der innerbetrieblichen Leistungsfähigkeit eine wachsende $\mathrm{Be}-$ deutung zukommt (vgl. Abbildung 19). Dieselbe Aussage gilt für die größenbezogenen Merkmale Bruttoeinnahmen, Betriebsausgaben und Umsätze (vgl. Abbildung 20). Die Verbindung dieser beiden Resultate läßt den Schluß zu, daß routinisierte Auftragsabwicklung bei hohen Warenumsätzen einer entsprechenden organisatorischen Grundlage innerhalb der Handelsvertretung bedarf ${ }^{1}$. Nach den vorliegenden Ergebnissen hat sich diese Ansicht vorwiegend bei relativ jungen Handelsvertreterunternehmen durchgesetzt, deren Inhaber oder Geschäftsführer ein Studium absolviert haben.

Diese Befunde widersprechen einem vielfach anzutreffenden Klischee vom Ein-Mann-Unternehmen und ständiger Reisetätigkeit; vielmehr wird deutlich, daß eine hinreichende innerbetriebliche Leistungsfähigkeit Grundlage langfristiger Erfolge sein muß. Handelsvertretungen, die diesem Anspruch (noch) nicht genügen, müssen ihre Position kritisch überdenken, um den hier erkennbaren Wandlungen in der Handelsvertretertätigkeit rechtzeitig $\mathrm{zu}$ begegnen (z.B. durch verstärkte Kooperation im organisatorischen Bereich).

1 Die Bedeutung des Telefonverkaufs betont Leicher, R., Telefon-Lektion für Handelsvertreter, in: VuM, Heft 10, 1980 , S. 64 f.. 


\begin{tabular}{|l|c|c|}
\hline \multicolumn{1}{|c|}{ ERKLÄRENDE VARIABLE } & $\begin{array}{c}\text { MULTIPLE } \\
\text { KORRELATION } \\
(\mathrm{r})\end{array}$ & $\begin{array}{c}\text { BEEINFLUSSUNGS- } \\
\text { RICHTUNG }\end{array}$ \\
\hline $\begin{array}{l}\text { AUFTRAGSABWICKLUNG MIT } \\
\text { TELEFON/TELEX (\%) }\end{array}$ & 0,38 & - \\
$\begin{array}{l}\text { ZAHL DER AUFTRÄGE PRO } \\
\text { WOCHE } \\
\text { ZAHL DER KUNDEN }\end{array}$ & 0,40 & - \\
\hline
\end{tabular}

ABB， 19: KUNDENBEZOGENE BESTIMMUNGSFAKTOREN DER ROLLENAUSÜBUNG IN DER INNERBETRIEBLICHEN LEISTUNGSFÄHIGKEIT

\begin{tabular}{|l|c|c|}
\hline \multicolumn{1}{|c|}{ ERKLÄRENDE VARIABLE } & $\begin{array}{c}\text { MULTIPLE } \\
\text { KORRELATION } \\
(r)\end{array}$ & $\begin{array}{c}\text { BEEINFLUSSUNGS- } \\
\text { RICHTUNG }\end{array}$ \\
\hline BRUTTOEINKOMMEN (DM) & 0.53 & - \\
$\begin{array}{l}\text { BETRIEBSAUSGABEN } \\
(\% \text { DER PROVISION) } \\
\text { VERMITTELTER WARENUMSATZ } \\
(\text { MIO.DM) }\end{array}$ & 0.56 & - \\
\hline
\end{tabular}

ABB, 20:GRÖSSENBEZOGENE BESTIMMUNGSFAKTOREN DER ROLLENAUSÜBUNG IN DER INNERBETRIEBLICHEN LEISTUNGSFÄHIGKEIT 


\subsection{Preisgestaltung und Leistungsvergütung}

Der preispolitische Handlungsspielraum von Handelsvertretungen gegenüber den Kunden erweist sich als nahezu invariant gegenüber einzelnen Gruppen unternehmensbezogener Variablen. Erst die gleichzeitige Analyse von Unternehmens- und Personenmerkmalen ermöglicht akzeptable Erklärungsbeiträge, die jedoch mit 10,8 \& nicht sehr hoch ausfallen. Noch schwächere Zusammenhänge ergeben sich bei der Leistungsvergütung mit nur 8 \& erklärter Varianz.

Betrachtet man die Variablen, die die Rollenausübung im Bereich der Preisgestaltung determinieren, so lassen die Ergebnisse die Schlußfolgerung zu, daß Ermessensspielräume für Preis- und Konditionenverhandlungen eher bei größeren Handelsvertreterunternehmen anzutreffen sind als bei kleineren. Dies wird beispielhaft aus Abbildung 21 erkennbar, wo eine niedrige Preis- und Konditionenverantwortlichkeit eher mit geringen Auftragszahlen, weniger Vertretungen und niedrigen Bruttoeinnahmen in Verbindung steht. Die Einbeziehung der Alleininhaber von Handelsvertretungen als erklärungswirksames Merkmal verstärkt diesen Eindruck. Die beschriebene relativ wenig ausgeprägte Rolle von Handelsvertretungen ist ferner vorwiegend in der Konsumgüterbranche im südwest- und süddeutschen Raum anzutreffen.

Eine relativ niedrige Ausprägung von Leistungen bei kleineren Unternehmen und umgekehrt höherem Leistungsangebot bei größeren ist ebenfalls bei der Leistungsvergütung $\mathrm{zu}$ erkennen. Hier belegen die als erklärungswirksam herausgefilterten Variablen (vgl. Abbildung 22), daß eher ältere Handelsvertretungen mit einem großen Kundenstamm und teilweise routinisierter Auftragsabwicklung Provisionskalkulationen vornehmen bzw. die Vergütung zusätzlicher Leistungen 


\begin{tabular}{|l|c|c|}
\hline ERKLÄRENDE VARIABLE & $\begin{array}{c}\text { MULTIPLE } \\
\text { KORRELATION } \\
(\mathrm{r})\end{array}$ & $\begin{array}{c}\text { BEEINFLUSSUNGS- } \\
\text { RICHTUNG }\end{array}$ \\
\hline ALLEININHABER & 0.16 & + \\
HESSEN-RHEINL.-PFALZ-SAAR & 0.21 & - \\
KONSUMGÜTER & 0.24 & + \\
BETRIEBSAUSGABEN (\% DER PROV.) & 0.26 & + \\
ZAHL DER AUFTRÄGE PRO WOCHE & 0.27 & - \\
LEHRE & 0.28 & - \\
STUDIUM & 0.29 & + \\
GESAMTZAHL DER VERTRETUNGEN & 0.30 & - \\
BRUTTOEINNAHMEN (DM) & 0.31 & - \\
MEHRERE WARENGRUPPEN & 0.32 & - \\
BAYERN & 0.33 & + \\
\hline
\end{tabular}

ABB , 21: UNTERNEHMENS- UND PERSONENBEZOGENE BESTIMMUNGSFAKTOREN DER ROLLENAUSÜBUNG IN DER PREISGESTALTUNG

\begin{tabular}{|l|c|c|}
\hline \multicolumn{1}{|c|}{ ERKLÄRENDE VARIABLE } & $\begin{array}{c}\text { MULTIPLE } \\
\text { KORRELATION } \\
(\mathbf{r} \text { ) }\end{array}$ & $\begin{array}{c}\text { BEEINFLUSSUNGS- } \\
\text { RICHTUNG }\end{array}$ \\
\hline STUDIUM & 0.12 & - \\
B - HH - HB - SCHLESWIG & 0.17 & - \\
MITTLERE GEBIETSGRÖSSE & 0.21 & - \\
ZAHL DER KUNDEN & 0.24 & + \\
INDUSTR, GE- UND VERBRAUCH & 0.26 & - \\
ALTER DER HANDELSVERTRETUNG & 0.27 & - \\
AUFTRAGSABWICKLUNG MIT & 0.28 & + \\
TELEFON/TELEX (\%) & & \\
\hline
\end{tabular}

ABB， 22: UNTERNEHMENS- UND PERSONENBEZOGENE BESTIMMUNGSFAKTOREN DER ROLLENAUSÜBUNG IN DER LEISTUNGSVERGÜTUNG 
zumindest durchzusetzen versuchen. Ansatzpunkte einer solchen aktiven Beeinflussung der Leistungsvergütung sind dabei vor allem bei Handelsvertretern mit Studienabschluß festzustellen. Ausgangspunkt dieser Entwicklung ist tendenziell die Branche industrieller $\mathrm{Ge}$ - und Verbrauchsgüter in Norddeutschland.

\subsection{Vertretungsprogramm und Produktanregungen}

Für die Erklärung von Aktivitäten bei der Zusammensetzung des Vertretungsprogramms von Handelsvertretungen lassen sich nur in geringem Maße unternehmensbezogene Prediktoren abgrenzen (vgl. Abbildung 17). Dabei fällt besonders auf, daß kundenorientierte Merkmale (z.B. Umfang des Kundenstammes, Abnehmergruppen) keinerlei Einfluß auf Maßnahmen im Rahmen der Gestaltung des Leistungsangebotes von Handelsvertretungen aufweisen. Der hier erkennbaren Tendenz einer mangelnden Kundenorientierung der gesamten Unternehmenstätigkeit unterliegen dabei vor allem kleinere Handelsvertretungen.

Abbildung 23 ermöglicht eine präzisere Umschreibung dieser Handelsvertretungen. Die aufgeführten Variablen belegen, daß ein wenig marktorientiertes Vertretungsprogramm anzutreffen ist bei jüngeren Unternehmen der Nahrungs- und Genußmittelbranche mit relativ geringem Umsatzvolumen. Die Zahl der Vertretungen ist insgesamt gering; eine der Vertretungen stellt i.d.R. eine Hauptvertretung dar, d.h. sie erbringt mehr als 50 \& der gesamten Provisionseinnahmen. Dieser Unternehmenstyp ist nach den vorliegenden Resultaten in weitgehend großflächigen Gebieten Süddeutschlands tätig. 


\begin{tabular}{|l|c|c|}
\hline \multicolumn{1}{|c|}{ ERKLÄRENDE VARIABLE } & $\begin{array}{c}\text { MULTIPLE } \\
\text { KORRELATION } \\
(\mathrm{r})\end{array}$ & $\begin{array}{c}\text { BEEINFLUSSUNGS- } \\
\text { RICHTUNG }\end{array}$ \\
\hline GESAMTZAHL DER VERTRETUNGEN & 0.13 & - \\
NAHRUNGS- UND GENUSSMITTEL & 0.18 & + \\
ALTER DER HANDELSVERTRETUNG & 0.21 & + \\
BAYERN & 0.23 & + \\
ZAHL DER WARENGRUPPEN & 0.25 & - \\
GROSSFLÄCHIGES GEBIET & 0.27 & - \\
VERMITTELTER WARENUMSATZ & 0.28 & + \\
HAUPTVERTRETUNG VORHANDEN & 0.29 & + \\
\hline
\end{tabular}

ABB， 23: UNTERNEHMENSBEZOGENE BESTIMMUNGSFAKTOREN DER ROLLENAUSÜBUNG BEIM VERTRETUNGSPROGRAMM

Diese Kennzeichnung von Handelsvertretungen läßt die Schlußfolgerung möglich erscheinen, daß Handelsvertreter kleinerer Unternehmen wenig marktorientiert agieren. Wenn dieser Sachverhalt in Einzelfällen zutreffen mag, deuten einige Indikatoren auf eine andere Interpretation. Das Vorhandensein einer Hauptvertretung und die Tatsache, daß es sich vorwiegend um jüngere Unternehmen handelt, unterstützen vielmehr die Argumentation, daß im vorliegenden Fall eher Unternehmen skizziert wurden, die sich noch in der Anfangsphase ihrer Tätigkeit befinden. Die wesentliche Stütze des Unternehmens bildet eine zentrale Vertretung, und die damit verbundene Abhängigkeit steht einer stärker marktorientierten Ausrichtung des Vertretungsprogramms (noch) entgegen ${ }^{1}$. Mit wachsender Größe des Unter-

1 Diesen Sachverhalt diskutiert aus Herstellersicht Bethge, K.M., Mit Handelsvertretern erfolgreich verkaufen, in: MA 1977 , S. $67 \mathrm{ff.}$. 
nehmens schwächt sich diese Tendenz ab, d.h. größere Handelsvertretungen orientieren die zusammensetzung ihres Vertretungsangebotes stärker an den Markterfordernissen.

Die allmähliche Profilierung einer eigenständigen Handelsvertretertätigkeit schlägt sich bei den Produktanregungen deutlich nieder. Anstöße zur Entwicklung neuer Produkte bzw. Vorschläge zur Sortimentsgestaltung der vertretenen Unternehmen stehen in hohem Zusammenhang mit kundenbezogenen Merkmalen. Abbildung 24 stellt dabei insbesondere die Erklärungswirksamkeit auftragsbezogener Merkmale heraus.

\begin{tabular}{|l|c|c|}
\hline \multicolumn{1}{|c|}{ ERKLÄRENDE VARIABLE } & $\begin{array}{c}\text { MULTIPLE } \\
\text { KORRELATION } \\
(\mathrm{r})\end{array}$ & $\begin{array}{c}\text { BEEINFLUSSUNGS- } \\
\text { RICHTUNG }\end{array}$ \\
\hline $\begin{array}{l}\text { AUFTRAGSABWICKLUNG MIT TELEFON/ } \\
\text { TELEX (\%) }\end{array}$ & 0.22 & + \\
ZAHL DER AUFTRÄGE PRO WOCHE & 0.24 & - \\
HÄNDLER & 0.26 & - \\
HANDWERKER - ENDVERBRAUCHER & 0.27 & - \\
\hline
\end{tabular}

ABB. 24: KUNDENBEZOGENE BESTIMMUNGSFAKTOREN DER ROLLENAUSÜBUNG BEI DEN PRODUKTANREGUNGEN

Die Art der Auftragsabwicklung und das Auftragsvolumen weisen auf den ersten Blick eine gegenläufige Tendenz auf. Anregungen zur Produkt- und Sortimentsgestaltung sind 
niedriger ausgeprägt, je mehr Aufträge ohne unmittelbaren Kundenbesuch erlangt wurden, d.h. je höher der Anteil telefonischer Aufträge ist. Telefonische Auftragsabwicklung läßt auf ein hohes Auftragsvolumen schließen, in der Konsequenz müßte eine große Anzahl vermittelter Aufträge ebenfalls zu geringen Aktivitäten im Bereich der Produktund Sortimentsanregungen führen.

Die Ergebnisse der Studie belegen jedoch eine umgekehrte Tendenz. Je höher die Anzahl der vermittelten Aufträge ist, umso ausgeprägter werden die Aktivitäten der Handelsvertretungen im Hinblick auf Produktanregungen. Dieser scheinbare Widerspruch erklärt sich aus den verschiedenen Möglichkeiten einer Auftragserlangung. Je nachdem ob Aufträge durch persönliche Kontakte mit dem Kunden - d.h. durch persönlichen Besuch - oder durch unpersönliche Medien telefonische Kontakte, Fernschreiber - erlangt werden, resultieren daraus Unterschiede bei den Aktivitäten in der Produkt- und Sortimentspolitik.

Die Resultate deuten an, daß die unmittelbare persönliche Kommunikation mit dem Kunden eher geeignet ist, von seiten des Marktes Anregungen zur Gestaltung von Produkten und Sortimenten aufzunehmen, als dies bei unpersönlichen Kontakten der Fall ist. Angesichts der unbestrittenen Notwendigkeit zur Entwicklung von neuen Produkten ${ }^{1}$ und der Verantwortung von Handelsvertretungen für die Sortimente der vertretenen Unternehmen müssen Handelsvertreter einen KompromiB finden zwischen einer routinisierten, kostengün-

$1 \mathrm{Vgl}$. Koinecke, J., Marketing-Praxis mit Handelsvertretern, a.a.0., S. $38 \mathrm{f}$; Hunsinger, H., Durch bessere Information erfolgreicher an den Handel verkaufen, Nr. 7 der Information zur Rationalisierung der Kommunikation, Frankfurt 1979. 
stigeren Auftragserlangung und intensiverem persönlichen Einsatz zur Aufdeckung von Marktchancen.

\subsection{Zusammenfassende Hypothesenbeurteilung}

Die Analyse der Rollenausübung in allen Dienstleistungsbereichen läßt zusammenfassend ein heterogenes Erscheinungsbild von Handelsvertretungen erkennen. Aus den in die Untersuchung einbezogenen Bestimmungsfaktoren der Rollenausübung hat sich keine Merkmalsgruppe oder eine einzelne Variable als dominant erwiesen. Auch erreichte die Höhe der Erklärungsbeiträge nur Werte zwischen $8 \&$ und $21 \%$ erklärter Varianz; lediglich die innerbetriebliche Leistungsfähigkeit konnte zu ca. $45 \&$ durch die Determinanten erklärt werden.

Eine zusammenfassende Beurteilung der Hypothesen zur Rollenausübung von Handelsvertretungen veranschaulicht $\mathrm{Ab}-$ bildung 25. Eindeutig bestätigt hat sich die Intergruppenhypothese, d.h. unternehmensbezogene Merkmale sind bei den oben diskutierten Einschränkungen - durchweg besser in der Lage, die Rollenausübung von Handelsvertretungen in allen Dienstleistungsbereichen zu erklären als personenbezogene Merkmale. Der hypostasierte dominante Einfluß abnehmerorientierter Merkmale konnte dagegen nur für den Leistungsbereich Produktanregungen nachgewiesen werden; für alle anderen Dienstleistungsbereiche ist die Intragruppenhypothese abzulehnen.

Uneinheitlich zeigen sich die Befunde zur Kombinationshypothese. Die Verknüpfung von unternehmens- und personenbezogenen Merkmalen führt nur teilweise zu einer Verbesserung des Erklärungswertes; insbesondere die Bereiche 


\begin{tabular}{|c|c|c|c|}
\hline Dienstleistungsbereich & $\begin{array}{l}\text { Intergruppenhypothese } \\
\qquad \mathrm{H}_{1}{ }^{\mathrm{HV}}\end{array}$ & $\begin{array}{l}\text { Intragruppenhypothese } \\
\qquad \mathrm{H}_{2}^{\mathrm{HV}}\end{array}$ & $\begin{array}{c}\text { Kombinationshypothese } \\
\mathrm{H}_{3}{ }^{\mathrm{HV}}\end{array}$ \\
\hline Informationsaustausch & $(+)$ & - & $(+)$ \\
\hline $\begin{array}{l}\text { innerbetriebliche Leistungs- } \\
\text { fähigkeit }\end{array}$ & + & - & + \\
\hline Preisgestaltung & + & - & + \\
\hline Produktanregungen & + & + & - \\
\hline Leis tungsvergütung & + & - & $(-)$ \\
\hline Vertretungsprogramm & + & $(-)$ & + \\
\hline
\end{tabular}

Abb. 25: Zusammenfassende Hypothesenbeurteilung zur Rollenausübung von Handelsvertretungen $+\quad$ : Hypothese bestätigt

- : Hypothese nicht bestätigt

(t) : Hypothese schwach bestätigt/abgelehnt 
Produktanregungen und Leistungsvergütung erweisen sich als invariant gegenüber der Variablenkombination.

Für das Marketing von Handelsvertretungen ist es daher angezeigt, die bei den einzelnen Dienstleistungsbereichen herausgearbeiteten unternehmensbezogenen Bestimmungsgrößen als relevante Determinanten der Rollenausübung im Rahmen der Maßnahmenplanung zu antizipieren. Voraussetzung dieser Vorgehensweise ist die Einbeziehung der Vorstellungen und Meinungen von seiten der Marktpartner. Im folgenden werden dabei zunächst die Rollenkomponenten der vertretenen Unternehmen diskutiert.

\subsection{Rollenerfüllung und -erwartung im Urteil der vertre- tenen Unternehmen}

Das Grundmodell einer Rollenanalyse im HandelsvertreterMarketing hat für die vertretenen Unternehmen von Handelsvertretungen die Beurteilung des gegenwärtigen Leistungsangebotes (Rollenerfüllung) und das Anspruchsniveau in einzelnen Dienstleistungsbereichen (Rollenerwartung) als relevante Rollenkomponenten abgegrenzt. Zur Ableitung von Maßnahmen ist für Handelsvertreter die Frage von unmittelbarer Bedeutung, ob im Hinblick auf das eigene Leistungsangebot Merkmale der vertretenen Unternehmen identifiziert werden können, die einen Einfluß auf die Beurteilung ihrer gegenwärtigen Leistungsausübung sowie das erwartete Leistungsniveau haben. Die Kenntnis derartiger Bestimmungsfaktoren versetzt Handelsvertreter in die Lage, mögliche Reaktionen und Verhaltensweisen von Herstellern - bei Kenntnis der als erklärungsunwirksam abgegrenzten Merkmale besser abschätzen zu können und somit die eigenen Aktivitäten darauf einstellen $z u$ können. Im folgenden werden 
daher zunächst die Bestimmungsfaktoren von Rollenerfüllung und -erwartung im tberblick vorgestellt, um auf dieser Grundlage die Besonderheiten einzelner Dienstleistungsbereiche aufzudecken ${ }^{1}$.

3.21 Bestimmungsfaktoren von Rollenerfüllung und-erwartung im Uberblick

Die Uberprüfung der Hypothesen über den Einfluß möglicher Bestimmungsfaktoren auf die Beurteilung des gegenwärtigen Leistungsangebotes von Handelsvertretungen sowie das Anspruchsniveau der Hersteller erfolgte analog $\mathrm{zu}$ den Handelsvertretungen auf regressionsanalytischer Basis ${ }^{2}$. Der Unterscheidung zwischen Rollenerfüllung und Rollenerwartung wurde durch eine Verwendung der beiden hierzu ermittelten Faktorenstrukturen als abhängigen Variablen Rechnung getragen ${ }^{3}$. Diese Vorgehensweise führt in Verbindung mit den unterschiedlichen Gruppen der unabhängigen Variablen $\mathrm{zu}$ einer Reihe von Regressionsgleichungen. Abbildung 26 und 27 dokumentieren die wesentlichen Resultate der Auswertungen zur Hypothesenprüfung im Uberblick.

1 Auch an dieser Stelle wird - wie bei den Handelsvertretungen - darauf verzichtet, die einfachen Ausprägungen von Rollenerfüllung und -erwartung sowie der unabhängigen Merkmale vorzustellen. Diesen deskriptiven Ubberblick vermitteln die Tabellen 3,4,5 im Anhang I.

2 Zur Vorgehensweise vgl. Kapitel C. 3.11 dieser Arbeit.

3 Zur Zusammensetzung dieser zentralen Dienstleistungsbereiche vgl. Kapitel C. 2.21 dieser Arbeit. 


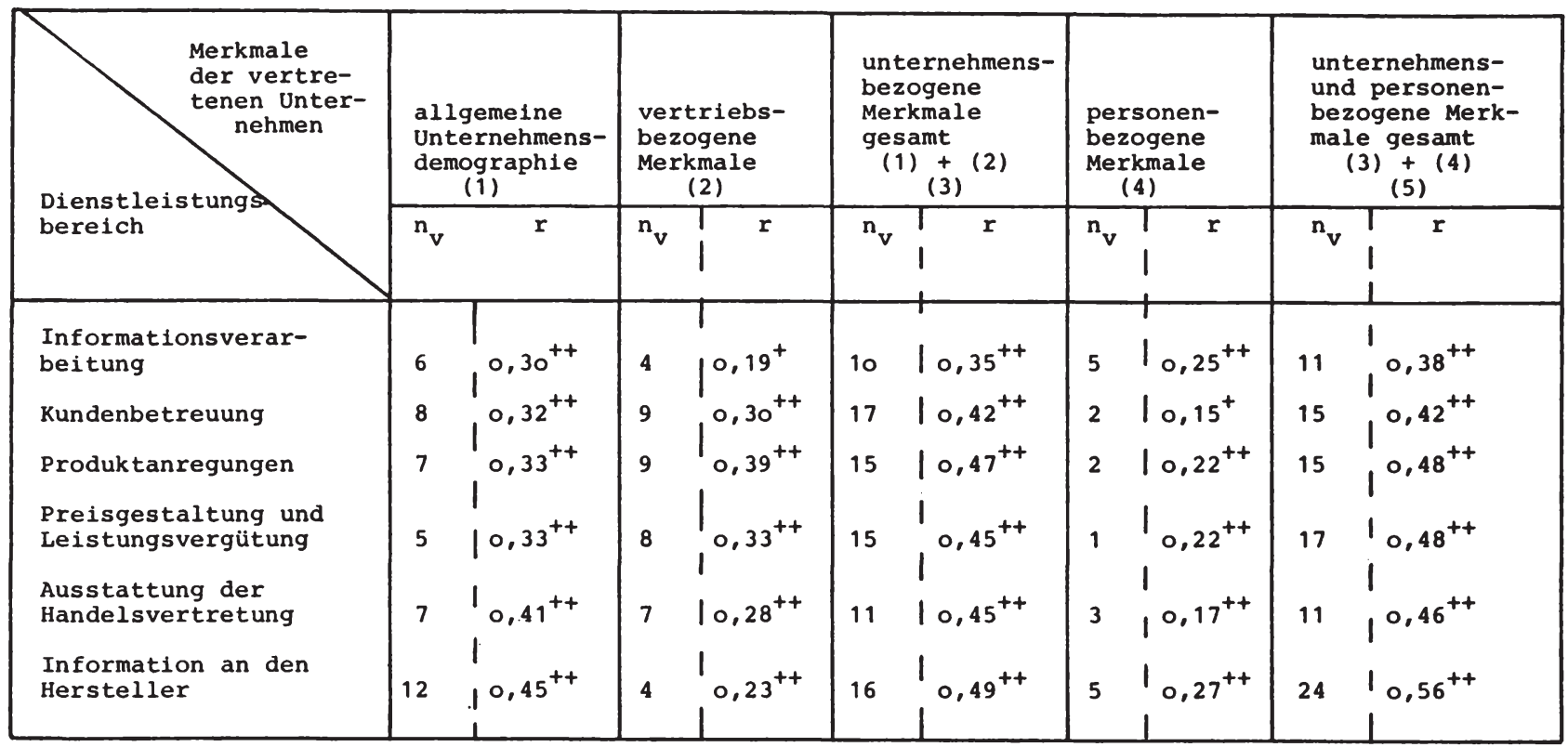

Abb. 26: Bestimmungsfakțoren der Rollenerfüllung von Handelsvertretungen aus der Sicht der Hersteller im Uberblick

$\mathrm{n}_{\mathrm{v}}$ : Anzahl der in die multiplen Regressionen einbezogenen Variablen

$r$ : multipler Korrelationskoeffizient

++99 \& - Signifikanzniveau

$+\quad: 958$ - Signifikanzniveau 


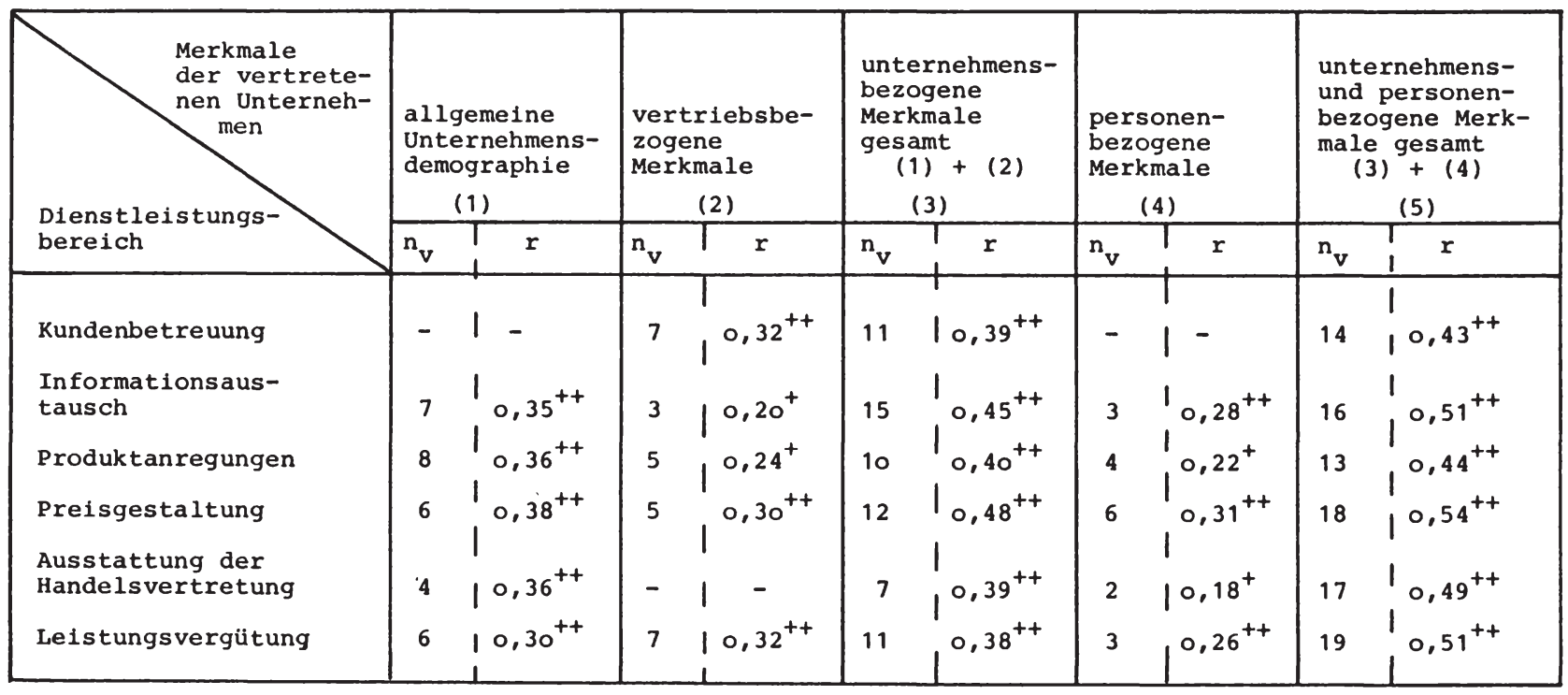

Abb. 27: Bestimmungsfaktoren der Rollenerwartung an Handelsvertretungen durch die Hersteller im Uberblick

$n_{v}:$ Anzahl der in die multiplen Regressionen einbezogenen Variablen

$r$ : multipler Korrelationskoeffizient

$++\quad$ : 998 - Signifikanzniveau

$+\quad$ : 958 - Signifikanzniveau 
Nach den vorliegenden Ergebnissen der Studie können sowohl die Beurteilung des Leistungsangebotes von Handelsvertretungen durch die Hersteller, d.h. die Rollenerfüllung, als auch die Rollenerwartungen bei globaler Betrachtung zu einem erheblichen Anteil durch unternehmens- und personenbezogene Merkmale der vertretenen Unternehmen erklärt werden. Ein Vergleich (der Spalten 5) zeigt, daß die erklärungswirksamen Merkmale bei der Rollenerfüllung multiple Korrelationen zwischen 0,38 und 0,56 und bei der Rollenerwartung zwischen 0,43 und 0,54 je nach Dienstleistungsbereich aufweisen.

Eine Gegenüberstellung unternehmens- und personenbezogener Merkmale (Spalten 3 und 4 ) belegt auch für die Ebene der vertretenen Unternehmen eine Dominanz der unternehmensorientierten Merkmale. Die Einbeziehung personaler Merkmale der Kontaktpartner von Handelsvertretern in den vertretenen Unternehmen ist dagegen in der Lage, den alleinigen Erklärungswert der unternehmensbezogenen Merkmale zu erhöhen (Spalten 3 und 5). Dabei sind diese Erklärungszuwächse bei den Rollenerwartungen erheblich höher als bei der Beurteilung der Rollenerfüllung. Bei der Rollenerfüllung führt die Einbeziehung personaler Merkmale lediglich für die herstellergerichteten Informationen $\mathrm{zu}$ einer Verbesserung der Korrelation von 0,47 auf 0,56; bei den Rollenerwartungen treten mehrere derartige Steigerungen auf - so beispielsweise beim Informationsaustausch $(0,45$ auf 0,51$)$, der Preisgestaltung $(0,48$ auf 0,54$)$ und insbesondere der Leistungsvergütung $(0,38$ auf 0,51$)$.

Diese generellen Unterschiede sowie eine wenig eindeutige Tendenz hinsichtlich der Erklärungsbeiträge innerhalb der unternehmensbezogenen Merkmale (Spalten 1 und 2) sollen durch eine differenziertere Analyse der einzelnen Dienstleistungsbereiche weiter erhellt werden. 
3.22 Bestimmungsfaktoren von Rollenerfüllung und-erwartung in einzelnen Dienstleistungsbereichen

\subsection{Informationsaustausch}

Bei der Generierung zentraler Dienstleistungsbereiche von Handelsvertretungen wurden bei der Beurteilung der Rollenerfüllung durch die Hersteller die Informationsverarbeitung durch Handelsvertretungen sowie die an das vertretene Unternehmen übermittelten Informationen als zwei getrennte Leistungsbereiche identifiziert, während diese Unterscheidung bei den Rollenerwartungen nicht festzustellen war. Die Frage, ob diese Unterscheidung durch unterschiedliche Bestimmungsfaktoren eine Bestätigung erfährt, muß weitgehend verneint werden. Zwar haben sich bei der Informationsverarbeitung sowie den herstellergerichteten Informationen im Rahmen der Rollenerfüllung verschiedene Merkmale als erklärungswirksam herausgestellt, diese betreffen jedoch vorwiegend die allgemeine Unternehmensdemographie, insbesondere die Rechtsform und regionale Merkmale.

Darüber hinaus dominieren zur Erklärung von Informationsaktivitäten sowohl bei der Rollenerfüllung als auch bei den Rollenerwartungen die allgemeinen Merkmale der vertretenen Unternehmen die vertriebsbezogenen Einflußgrößen. Demnach müssen Handelsvertreter ihren Informationsaustausch mit den vertretenen Unternehmen mehr an den generellen Merkmalen der vertretenen Unternehmen als an den spezifischen 
vert́riebsorientierten Gegebenheiten ausrichten. Welche besonderen Faktoren dabei zu beachten sind, belegt die Analyse der erklärungswirksamen Merkmale für die Erwartungen der Hersteller im Rahmen des Informationsaustausches.

Abbildung 28 umfaßt die 15 Merkmale, die sich für den Informationsaustausch mit einem multiplen Korrelationskoeffizienten von 0,45 als relevant eriwesen haben. Demnach lassen sich Aussagen zur Verwertung von Hersteller- und Kundeninformationen sowie zur Informationsübermittlung an die Marktpartner vor allem für die Investitionsgüterindustrie ableiten. Die Anforderungen an Handelsvertretungen sind dabei besonders hoch bei eher kleineren Herstellern, die mit einer geringen $\mathrm{Zahl}$ von Beschäftigten ein relativ kleines Produktprogramm an die weiterverarbeitende Industrie verkaufen ${ }^{1}$. Unternehmen, die mit wenigen Handelsvertretungen zusammenarbeiten, stellen insgesamt geringere Anforderungen; dasselbe gilt, wenn sich die Zusammenarbeit bereits auf einen längeren Zeitraum erstreckt.

Diese gering ausgeprägten Erwartungshaltungen der beschriebenen Hersteller lassen in ihrer Gesamtheit auf ein wenig flexibles Agieren am Markt schließen. Bei derartigen Vertretungen ist $\mathrm{zu}$ überprüfen, ob diese wenig kundenbezogenen Verhaltensweisen langfristig die Aufrechterhaltung der vertraglichen Beziehungen lohnen ${ }^{2}$.

1 Ähnliche Resultate belegen Untersuchungen des RKW neben der Investitionsgüterbranche in der Tendenz für weitere Wirtschaftsbereiche. Vgl. RKW (Hrsg.), Marketing in mittleren und kleinen Industrieunternehmen dargestellt am Beispiel der Wohnmöbelindustrie, Frankfurt 1979, S. 54 f.; ders., ... dargestellt am Beispiel der Bekleidungsindustrie, Frankfurt 1976 , S. 25 ff..

2 Speziell dieser Unternehmensgruppe sind die potentiellen Risiken mangelnder Informationspolitik aufzuzeigen; vgl. Höfner, K., Leitfaden für das praktische Marketing in mittleren und kleinen Unternehmen der Investitions- und Gebrauchsgüterindustrie, Frankfurt 1978,s.26 ff.; aus Handelsvertretersicht belegt diese Argumentation Bless,H.J., Unwirtschaftlichkeit wider Willen, in: HV+HM 1976,S.451. 


\begin{tabular}{|c|c|c|}
\hline ERKLÄRENDE VARIABLE & $\begin{array}{c}\text { MULTIPLE } \\
\text { KORRELATION } \\
(r)\end{array}$ & $\begin{array}{l}\text { BEEINFLUS- } \\
\text { SUNGSRICHTUNG }\end{array}$ \\
\hline INVESTITIONSGÜTER & 0,18 & - \\
\hline NUR HANDELSVERTRETER & 0.23 & + \\
\hline ZAHL DER WARENGRUPPEN & 0,26 & + \\
\hline ANZAHL DER BESCHÄFTIGTEN & 0.29 & + \\
\hline ANZAHL DER ZWEIGWERKE & 0,31 & - \\
\hline ABSATZ AN WEITERVERARBEITER & 0.34 & - \\
\hline HERSTELLERUNTERNEHMEN & 0,35 & - \\
\hline WENIGE WARENGRUPPEN & 0,37 & + \\
\hline $\begin{array}{l}\text { RÄUMLICHE HV-VERTRIEBSORGANI- } \\
\text { SATION }\end{array}$ & 0,39 & - \\
\hline $\begin{array}{l}\text { DAUER DER ZUSAMMENARBEIT MIT } \\
\text { HV }\end{array}$ & 0.40 & + \\
\hline GESAMTZAHL DER HV & 0.41 & - \\
\hline SORTIMENTSANTEIL DER REISENDEN & 0.42 & - \\
\hline UMSATZANTEIL DER REISENDEN & 0.43 & - \\
\hline UMSATZANTEIL DER HV & 0.44 & + \\
\hline $\begin{array}{l}\text { PRODUKT- UND KUNDENBEZOGENE HV- } \\
\text { VERTRIEBSORGANISATION }\end{array}$ & 0.45 & + \\
\hline
\end{tabular}

ABB , 28: UNTERNEHMENSBEZOGENE BESTIMMUNGSFAKTOREN DER ROLLENERWARTUNG DURCH DIE HERSTELLER BEIM INFORMATIONSAUSTAUSCH 
Eine besondere Herausforderung für Handelsvertretungen zeigt sich im Rahmen der Vertriebsstruktur der gekennzeichneten Unternehmen. Eine Möglichkeit zur Profilierung wird dabei in zweifacher Hinsicht deutlich. Auf der einen Seite belegen die vorliegenden Resultate besonders hohe Anforderungen an die Informationsaktivitäten, wenn das vertretene Unternehmen eher viele Handelsvertreter einsetzt. Ein entsprechendes Leistungsangebot in diesem Bereich trägt damit zur langfristigen Absicherung der Vertragsbeziehung bei.

Eine weitere wichtige Profilierungsmöglichkeit hat sich bei Herstellern mit gemischtem Vertrieb (d.h.Einsatz von Handelsvertretern und Reisenden) herausgestellt. Eine unerwartete Tendenz eröffnet hier den Handelsvertretungen besondere Chancen. Je mehr neben den Handelsvertretern von dem vertretenen Unternehmen auch Reisende im Vertrieb eingesetzt werden, um so höhere Anforderungen werden an die informationsbezogenen Leistungen der Handelsvertreter gestellt.

Neben dem Versuch der Hersteller, den Handelsvertretungen ein den Reisenden vergleichbares Berichtswesen abzuverlangen, belegt dieses Ergebnis die Vorteile der Handelsvertretungen als selbständige Dienstleistungsanbieter ${ }^{1}$. Gerade Hersteller, die einen großen Teil ihres Produktprogramms mit Reisenden absetzen - und diese damit zugleich hohe Umsatzbeiträge erbringen -,stellen an die Informationsaktivitäten ihrer Handelsvertretungen besondere Anforderungen. Es ist $z u$ vermuten, daß diese Anforderungen auf solche Informationen abzielen, die die Reisenden der Hersteller nicht bzw. nur kaum liefern können. Hier sind beispielhaft detaillierte Kenntnisse über Kunden und Branche zu nennen, die bei Handelsvertretern aufgrund der

1 Vgl. Willas, J., a.a.o., s. 8. 
längerfristigen Tätigkeit in demselben Gebiet tendenziell höher liegen als bei Reisenden. Eine gezielte Bearbeitung dieser Nische ermöglicht Handelsvertretungen eine Absicherung der Beziehungen zum Hersteller. Unabdingbare Voraussetzung dafür ist jedoch, daß die Handelsvertreter derartige Schwerpunkte ihrer Tätigkeit den vertretenen Unternehmen transparent machen, d.h. insbesondere Leistungen in der Abgrenzung zum konkurrierenden Reisenden müssen den vertretenen Unternehmen besser "verkauft" werden.

\subsection{Kundenbetreuung}

Die Betreuung der Kunden gilt nach vorliegenden Studien als wichtigstes Dienstleistungsmerkmal von Handelsvertretungen, wobei gerade die ständige Präsenz des Handelsvertreters in seinem Vertretungsgebiet besonders hervorgehoben wird ${ }^{1}$. Die Frage nach den gegenwärtigen Beurteilungen ihrer Leistungen in diesem Dienstleistungsbereich sowie den Erwartungen der Hers.teller ist demnach für die Maßnahmenplanung im Handelsvertreter-Marketing von herausragendem Interesse.

Der globale Überblick zur Beurteilung der Kundenbetreuung hinsichtlich der Rollenerfüllung und -erwartung belegt deutliche Unterschiede in den Erklärungsmöglichkeiten dieser Rollenkomponenten (vgl. Abbildung 26 und 27). Demnach läßt sich die Rollenerfüllung in diesem Dienstleistungsbereich durch Merkmale der vertretenen Unternehmen insgesamt besser erklären als die Rollenerwartungen.

1 Vgl. Tab. 5 , Anhang I sowie Meffert, H., Kimmeskamp, G., a.a.0., S. 69; Koinecke, J., Marketing-Praxis mit Handelsvertretern, a.a.0., S. 82 . 
Unterschiede zwischen Rollenerfüllung und -erwartung ergeben sich bei den unternehmensbezogenen Bestimmungsfaktoren. Während acht allgemeine unternehmensdemographische Merkmale die gegenwärtige Beurteilung der Kundenbetreuung erklären können, weist keines der Merkmale eine prognostische Relevanz für die Rollenerwartungen in diesem Bereich auf ${ }^{1}$. Ferner eignen sich vertriebsbezogene Merkmale der vertretenen Unternehmen - mit einer noch zu diskutierenden Ausnahme - vorwiegend zur Erklärung von Erwartungshaltungen; die Beurteilung aktueller Leistungen wird durch sie kaum beeinflußt.

Gemeinsamkeiten zwischen Rollenerfüllung und Rollenerwartung belegen die personenbezogenen Merkmale der Kontaktpartner von Handelsvertretungen in den vertretenen Unternehmen. Der Erklärungswert dieser Variablengruppe ist in beiden Fällen nur gering; auch kann der Erklärungswert unternehmensbezogener Faktoren durch die Hinzunahme personaler Faktoren nur bei den Rollenerwartungen geringfügig erhöht werden.

Ein für Handelsvertretungen beachtenswertes Einzelergebnis verdient eine gesonderte Erwähnung. Dies betrifft den Einfluß der Vertriebsstruktur auf die Beurteilung der Rollenerfüllung.

Die Analyse des Zusammenhangs zwischen vertriebsorientierten Merkmalen der vertretenen Unternehmen und der Kundenbetreuung hat bei der Beurteilung der Rollenerfüllung u.a. die Struktur des Vertriebes als Einflußkomponente herausgefiltert. Während die Mehrzahl der einbezogenen Variab-

1 Allerdings ist zu beachten, daß mit den genannten acht Merkmalen nur ein Varianzanteil von ca. 10 \& erklärt wird. 
len die Schlußfolgerung zuläßt, daß mit wachsender Bedeutung der Handelsvertretungen für den Hersteller - ausgedrückt durch die Umsatzbeiträge der Handelsvertreter - sowie einer längeren Zusammenarbeit die Beurteilung der Kundenbetreuung eher positiv ausfällt, muß diese Aussage hinsichtlich der Vertriebsstruktur modifiziert werden.

Greift man aus der Kundenbetreuung die Erweiterung des Kundenkreises als einzelne Leistungskomponente heraus, ergeben sich signifikante Unterschiede in der gegenwärtigen Beurteilung dieser Leistung in Abhängigkeit davon, ob das vertretene Unternehmen nur Handelsvertreter, oder Reisende und Handelsvertreter einsetzt. Abbildung 29 dokumentiert diesen Zusammenhang ${ }^{1}$.

31 \& der befragten Unternehmen setzen sowohl Handelsvertreter als auch angestellte Reisende im Vertrieb ein; 69 \& arbeiten nur mit Handelsvertretern. Bei der Beurteilung von Handelsvertretungen hinsichtlich der Erweiterung des Kundenkreises werden dagegen die Handelsvertretungen erheblich besser eingeschätzt, wenn das vertretene Unternehmen nicht mit Reisenden zusammenarbeitet. Anders ausgedrückt: Wenn ein Hersteller beide Vertriebsorgane einsetzt, werden Handelsvertreter hinsichtlich ihrer Leistungsfähigkeit zur Erweiterung des Kundenkreises deutlich niedriger eingestuft als bei Herstellern, die ihre Erzeugnisse ausschließlich mit Handelsvertretern absetzen.

Dieses Resultat birgt ein hohes $M a \beta$ an Brisanz in sich fur diejenigen Handelsvertretungen, die in derartigen gemischten Vertriebssystemen tätig sind. Je nach der strategischen Aus-

1 Zur Kreuztabellierung vgl. Nie, N.H., u.a., SPSS, a.a.O., S. $218 \mathrm{ff.;}$ der asymmetrische Koeffizient eta gibt Auskunft über die Stärke des Zusammenhangs zwischen einer nominalen und einer intervallskalierten Variablen $(0=$ keine, 1 = hohe Ubereinstimmung der Mittelwerte); vgl. ebenda, s. 230 . 


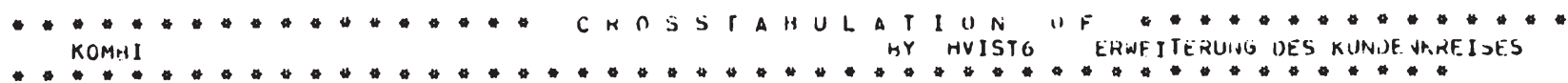

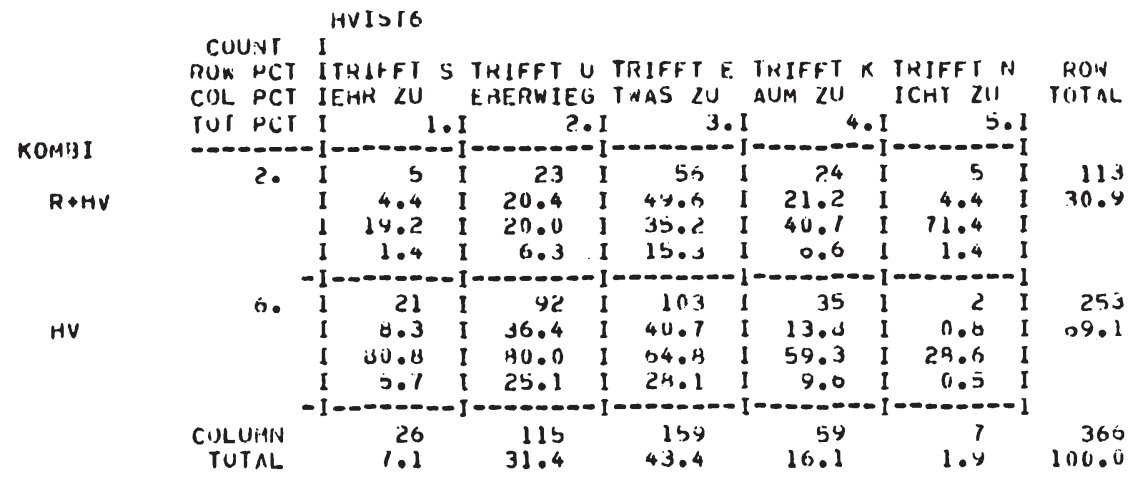

CHI SUISARE $=17.48171 \mathrm{NITH} 4$ UEGREES JF FHEEUOH SIONIFICAHCE $=0.0116$

ETA $=0.21 B 55 \mathrm{WITH}$ KO UI DEPEINIENT.

IUMBBEH OF MISSINSG UBSEKVATIOMS = J6

\section{Lesebeispiel:}

- 5 Unternehmen, die HV und Reisende einsetzen, sind in hohem Maße der Meinung, daß HV ihren Kundenkreis ständig erweitern.

- Dies entspricht einem Anteil von 4,4 aller Unternehmen mit gemischtem Vertrieb (HV und R)

- bzw. 19,2 der Unternehmen, die die Erweiterung des Kundenkreises in hohem Maße sehen

Abb. 29: Zusammenhänge zwischen der Vertriebsstruktur und der Rollenerfüllung hinsichtlich der Erweiterung des Kundenkreises 
richtung der Absatzpolitik des vertretenen Unternehmens sowie insbesondere dem Schwergewicht, das der Erweiterung des Kundenkreises beigemessen wird, droht hier den Handelsvertretungen langfristig eine Ablösung durch unternehmensangestellte Reisende. Für diese Interpretation spricht auch die Tatsache, daß sich der beschriebene Unterschied bei den Rollenerwartungen nicht fortsetzt.

Ein hinreichendes Ausmaß an Aktivitäten im Bereich der Neukundengewinnung ist damit als unverzichtbarer Bestandteil der Handelsvertreterleistungen anzusehen. Die Berücksichtigung dieser Erkenntnisse ist angesichts der außerordentlich hohen Anforderungen der Hersteller an die Kundenbetreuung ${ }^{1}$ in die Maßnahmenplanung von Handelsvertretungen einzubeziehen.

\subsection{Produktanregungen}

Die Mitwirkung von Handelsvertretungen bei den Produktund Sortimentsentwicklungen der vertretenen Unternehmen läßt sich bei globaler Betrachtung sowohl hinsichtlich der Rollenerfüllung als auch bei den Rollenerwartungen durch Merkmale der Hersteller differenzierter beschreiben (vgl. Abbildung 26 und 27). Dabei ist für die Rollenerfüllung ein Schwerpunkt bei den vertriebsbezogenen Merkmalen erkennbar, während die Rollenerwartung mehr durch allgemeine unternehmensdemographische Merkmale erklärt werden kann.

Betrachtet man zunächst die Beurteilung der Rollenerfüllung im Detail, so belegen die Ergebnisse, daß die Aktivitäten der Handelsvertreter bei der Vermittlung von Anregungen zur Produkt- und Sortimentsgestaltung vor allem von

1 Zur absoluten Ausprägung dieses Anforderungsniveaus vgl. Tab. 5 in Anhang $I$. 
solchen Herstellern tendenziell positiv eingeschätzt werden, die große Teile ihres Umsatzaufkommens mit Handelsvertretern realisieren. Steigt dagegen der Umsatzanteil anderer Vertriebswege - beispielsweise aus Direktgeschäften -, sehen sich die Handelsvertretungen einer erheblich niedrigeren Beurteilung in diesem Leistungsbereich konfrontiert (vgl. Abbildung 30). Auch die Dauer der Zusammenarbeit mit den vertretenen Unternehmen erweist sich nicht als Garant einer positiven Beurteilung im Bereich der Produktanregungen. Möglicherweise eingefahrene Verhaltensweisen bei langfristigen Geschäftsbeziehungen stehen nach diesen Resultaten einer - von den Herstellern offenbar vermiBten aktiven Ausgestaltung im Hinblick auf produkt- und sortimentspolitische Anregungen entgegen ${ }^{1}$.

Eine Differenzierung der Rollenerwartungen lenkt das Interesse auf eine Unterscheidung der Gegebenheiten einzelner Wirtschaftsbereiche. Eine Gegenüberstellung der Erwartungen im Hinblick auf die Vermittlung von Anregungen zur Entwicklung neuer Produkte belegt signifikante Unterschiede zwischen den verschiedenen Branchen. Abbildung 31 dokumentiert diese Zusammenhänge mit einer Fehlerwahrscheinlichkeit von weniger als 1 .

1 Initiativen, die durch Handelsvertreter ausgelöst werden, sind dabei erfolgversprechender als herstellerinduzierte Aktivitäten. So hat sich beispielsweise eine systematische Heranziehung von Außendienstmitarbeitern in diesem Problemzusammenhang als wenig sinnvoll erwiesen; vgl. Grace, D., Pointon, T., Marketing Research through the Salesforce, in: IMM 1980, S. $53 \mathrm{ff.}$. 


\begin{tabular}{|c|c|c|}
\hline ERKLÄRENDE VARIABLE & $\begin{array}{l}\text { MULTIPLE } \\
\text { KORRELATION } \\
(\mathrm{r})\end{array}$ & $\begin{array}{l}\text { BEEINFLUS- } \\
\text { SUNGSRICHTUNG }\end{array}$ \\
\hline $\begin{array}{l}\text { UMSATZANTEIL DER HANDELSVERTRE- } \\
\text { TER }\end{array}$ & 0.25 & - \\
\hline AUSSENLAGER BEI DEN HV & 0.28 & + \\
\hline $\begin{array}{l}\text { UMSATZANTEIL AUS DIREKTGESCHÄF- } \\
\text { TEN }\end{array}$ & 0.31 & + \\
\hline ZAHL DER HANDELSVERTRETERLÄGER & 0.32 & - \\
\hline $\begin{array}{l}\text { RÄUMLICHE VERTRIEBSORGANISATION } \\
\text { BEI REISENDEN }\end{array}$ & 0.34 & + \\
\hline SORTIMENTSANTEIL DER HV & 0,36 & + \\
\hline SORTIMENTSANTEIL DER REISENDEN & 0.37 & + \\
\hline DAUER DER ZUSAMMENARBEIT MIT HV & 0,38 & + \\
\hline $\begin{array}{l}\text { RÄUMLICHE VERTRIEBSORGANISATION } \\
\text { BEI HV }\end{array}$ & 0.39 & + \\
\hline
\end{tabular}

ABB . 30: VERTRIEBSBEZOGENE BESTIMMUNGSFAKTOREN DER ROLLENERFÜLLUNG VON HANDELSVERTRETUNGEN BEI DEN PRODUKTANREGUNGEN 


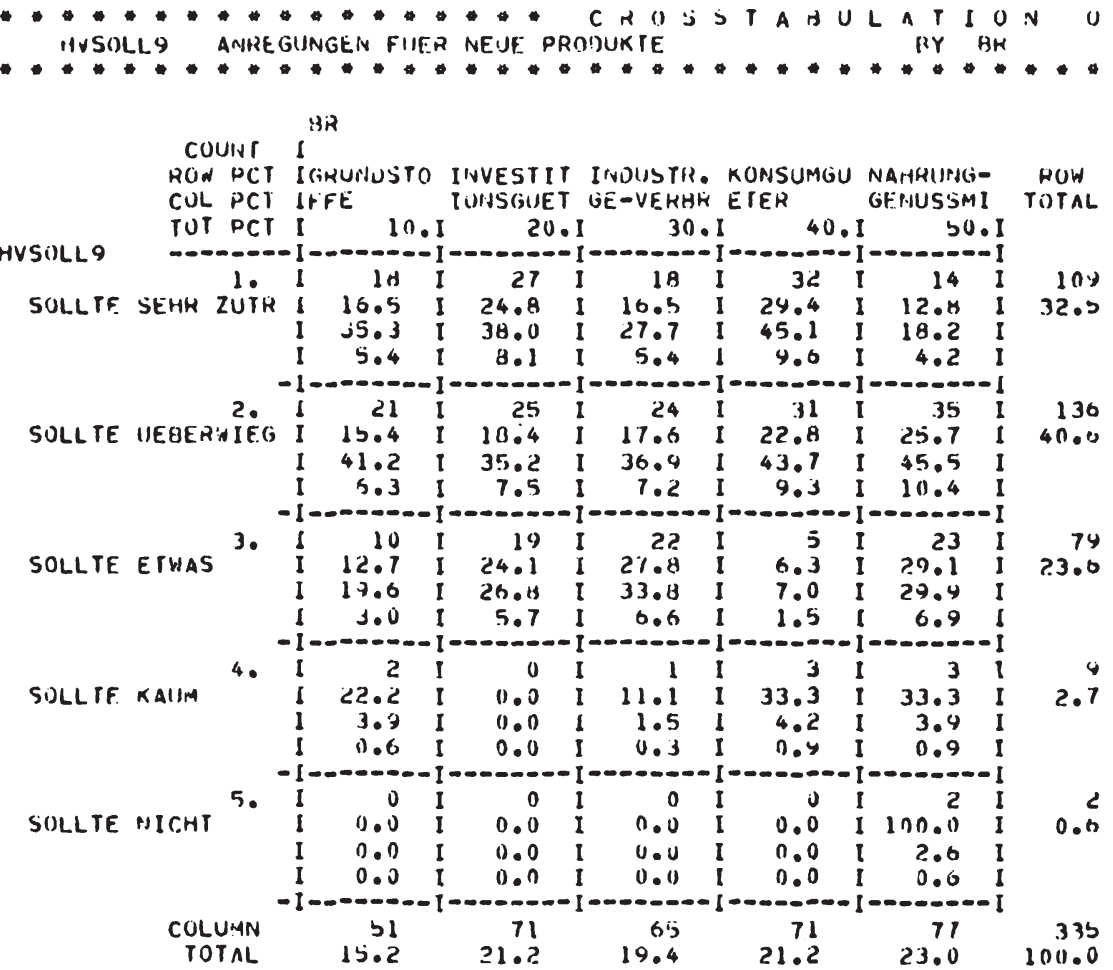

CHI SQUAAF $=34.22919$ WITH 16 IDEUREES UF FREEDOIA SISINIFICANCE = U.0N5I

ETA $=0.23712 \mathrm{WITH} \mathrm{HVSULLG} \mathrm{DERENISENT.}$

NUMBER OF MISSING UQSERVATIOIS = 07

ABH. 31: ZUCAMMFNHAFNGE ZWISCHEN DEN HERSTELLEDFFWAMTIMIGEN FUER

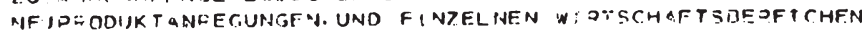


Im Durchschnitt erwarten $32,5 \&$ aller befragten Hersteller einen sehr hohen Umfang von Anregungen für ihre Neuproduktentwicklung. Erheblich höher liegen diese Anforderungen bei den Investitionsgütern mit 38 \& und vor allem bei den Konsumgütern mit 45 \%. Unterdurchschnittliche Erwartungen haben die Hersteller industrieller Ge- und Verbrauchsgüter $(28$ \%) und von Nahrungs- und Genußmitteln (18 \%).

Diese starke Polarisierung der einzelnen Wirtschaftsbereiche verlangt eine entsprechende Reaktion der jeweiligen Handelsvertretungen. Insbesondere angesichts kürzerer Produktlebenszyklen ${ }^{1}$ betreffen Entscheidungen der vertretenen Unternehmen in der Produkt- und Sortimentspolitik unmittelbar den Erfolg der Tätigkeit von Handelsvertretungen. Im Lichte dieser Entwicklung sollten auch Handelsvertreter der Nahrungs- und Genußmittelbranche prüfen, ob die relativ niedrigen Erwartungshaltungen ihrer vertretenen Unternehmen den Markterfordernissen genügen. Andernfalls müßte vor der Ubermittlung konkreter Anregungen für die Produktpolitik zunächst die allgemeine Sensibilität der Hersteller für die Probleme der Produkt- und Sortimentspolitik erhöht werden.

\subsection{Preisgestaltung und Leistungsvergütung}

Bei der Beurteilung des gegenwärtigen Leistungsangebotes von Handelsvertretungen haben sich Preisgestaltung und Leistungsvergütung als ein Dienstleistungsbereich herausgestellt, während bei den Rollenerwartungen eine Trennung beider Bereiche $z u$ verzeichnen war. Ohne die generellen Implikationen dieses Ergebnisses aufzugreifen, steht im folgenden

$1 \mathrm{Vgl}$. Meffert, H., Perspektiven des Marketing in den 8oer Jahren, a.a.O., S. 59.

2 Vgl. hierzu Kapitel C. 2.21 dieser Arbeit. 
die Frage im Mittelpunkt, ob unterschiedliche Bestimmungsfaktoren diese Differenzierung bestätigen und damit möglicherweise angepaßte Maßnahmen nach sich ziehen.

Die Einschätzung der Rollenerfüllung von Handelsvertretungen durch die Hersteller in den Aktivitäten der Preisgestaltung und Leistungsvergütung kann durch allgemeine unternehmensdemographische sowie vertriebsbezogene Merkmale gleichermaßen erklärt werden (vgl. Abbildung 26). Bei den als erklärungswirksam identifizierten Variablen tritt dabei insbesondere die Vertriebsstruktur als Unterscheidungsmerkmal hervor. Eine differenziertere Analyse dieses Ergebnisses führt zu teilweise überraschenden Aussagen.

Abbildung 32 dokumentiert den Zusammenhang zwischen der Vertriebsstruktur und der Beurteilung des Handlungsspielraums für Preisnachlässe, der den Handelsvertretern durch die Hersteller zugebilligt wird. Während lediglich 31 \& der vertretenen Unternehmen mit einem gemischten Vertrieb arbeiten, konzentrieren sich auf diese Gruppe 76 \% aller Handelsvertretungen mit einem relativ hohen Handlungsspielraum für Preisnachlässe. Dementsprechend entfallen auf die 69 \& der Unternehmen, die ausschließlich Handelsvertreter einsetzen, nur 24 \% der Handelsvertreter mit hohem Handlungsspielraum.

Diese Daten belegen eindrucksvoll, daß Hersteller mit gemischter Vertriebsstruktur ihren Handelsvertretern mehr Preisverantwortung zubilligen, als Hersteller, die ausschließlich Handelsvertreter einsetzen. Hinsichtlich der Frage nach den Gründen dieses Phänomens kann vermutet werden, daß Hersteller der letztgenannten Gruppe diesen 


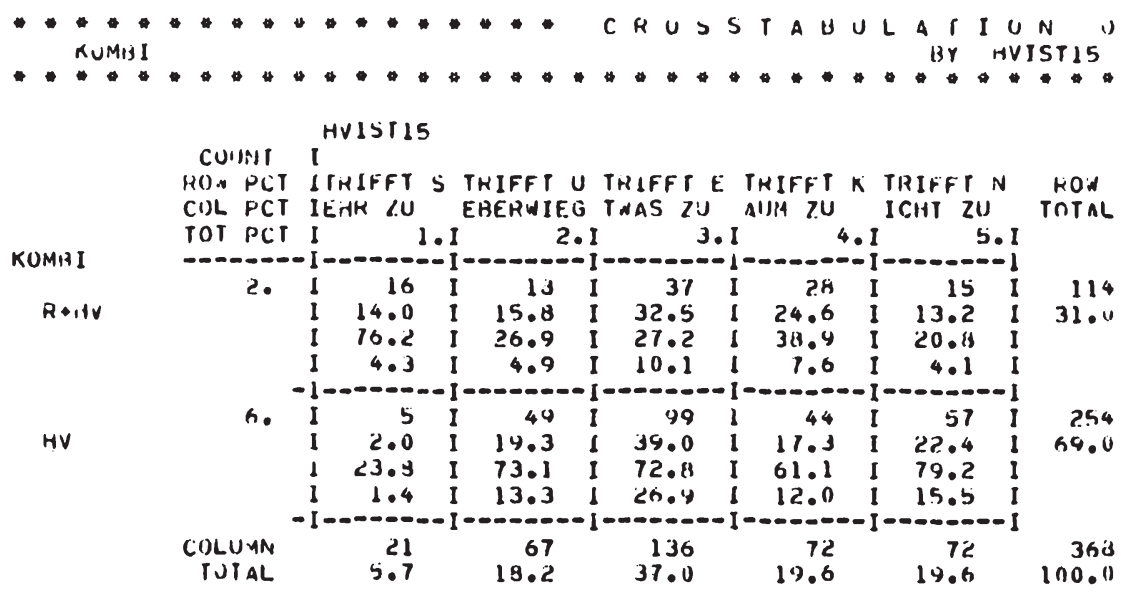

CHI SUUAME $=27.08449$ NITH 4 DEGREES OF FHEEDUA SIGINIFICANCE $=0.0000$

ETA $=0.27129$ NITH KOMMHI DEPENAENT.

HUMHEA OF MISJING JASERVATIIINS =

34

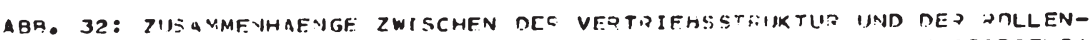

EPFIJFLLUNG HIY:ICHTLICH DFG DOFISONLITISCHFI H NOLUNGSSPIELQAIJMS 
unmittelbar erfolgswirksamen Entscheidungsbereich in eigener Verantwortung behalten wollen, Hersteller der ersteren Gruppe dagegen ihre Einfluß- und Kontrollmöglichkeiten durch die Reisenden in hinreichendem Maße realisiert sehen. Dieser spezielle Aspekt der Verteilung preispolitischer Verantwortlichkeiten erfährt auch bei einer Analyse der Erwartungshaltungen der Hersteller keine Änderung und festigt damit die geäußerte Vermutung.

Die Erwartungen der Hersteller an die Aktivitäten der Handelsvertreter im Rahmen der Preis- und Konditionengestaltung belegen einen herausragenden Einfluß personenbezogener Bestimmungsfaktoren (vgl. Abbildung 27). Dies betrifft sowohl den alleinigen Erklärungswert dieser Merkmalsgruppe (sechs Variable mit einer multiplen Korrelation von 0,31) als auch den Erklärungszuwachs bei Verknüpfung von unternehmens- und personenorientierten Faktoren (von 0,48 auf $0,54)$.

Eine Beantwortung der Frage, ob sich personale Merkmale der unmittelbaren Kontaktpartner von Handelsvertretern in den vertretenen Unternehmen maßnahmenbezogen umsetzen lassen, soll mit Hilfe der Abbildung 33 erfolgen. Die Abbildung belegt die ermittelten Zusammenhänge zwischen den Erwartungen der Hersteller hinsichtlich der Preis- und Konditionenpolitik von Handelsvertretungen und den personalen Merkmalen der für die Handelsvertreterkontakte zuständigen Personen. 


\begin{tabular}{|l|c|c|}
\hline \multicolumn{1}{|c|}{ ERKLÄRENDE VARIABLE } & $\begin{array}{c}\text { MULTIPLE } \\
\text { KORRELATION } \\
(\mathrm{r})\end{array}$ & $\begin{array}{c}\text { BEEINFLUSSUNGS- } \\
\text { RICHTUNG }\end{array}$ \\
\hline ALTER & 0.20 & + \\
ALLEININHABER-MITINHABER & 0.24 & - \\
LEHRE & 0.27 & - \\
DAUER DER AUSGEÜBTEN TÄTIGKEIT & 0.29 & + \\
UNTERNEHMENSZUGEHÖRIGKEIT & 0.30 & + \\
ANTEIL DER ARBEITSZEIT MIT HV & 0.31 & + \\
\hline
\end{tabular}

ABB. 33: PERSONENBEZOGENE BESTIMMUNGSFAKTOREN DER ROLLENERWARTUNG AN HANDELSVERTRETUNGEN BEI DER PREISUND KONDITIONENGESTALTUNG

Nach den vorliegenden Resultaten werden die Erwartungen an Handelsvertreter in diesem Leistungsbereich vorwiegend durch die Inhaber der vertretenen Unternehmen geprägt. Dabei neigen ältere Personen, die ihre gegenwärtig ausgeübte Tätigkeit im Unternehmen relativ lange ausführen bzw. die lange im Unternehmen tätig sind, zu tendenziell niedriger ausgeprägten Erwartungshaltungen. Dieses Resultat dokumentiert eine eher reservierte Haltung der vertretenen Unternehmen gegenüber einer weitreichenden Verantwortung von Handelsvertretern für die Preisgestaltung sowie die Vereinbarung der Konditionen. Solche Entscheidungen mit finanziellen Konsequenzen wollen sich die Hersteller bzw. Unternehmensinhaber weitgehend vorbehalten: 
Eine leichte Abschwächung dieser Tendenz deutet sich an, bezieht man das Ausmaß der Interaktion zwischen Hersteller und Handelsvertretung in die Betrachtung ein. Mit einer intensiveren Zusammenarbeit zwischen Handelsvertretung und vertretenem Unternehmen steigen die Erwartungen an die Leistungen in der Preis- und Konditionengestaltung. Gemessen über den Anteil der Arbeitszeit des Inhabers für die unmittelbaren Kontakte mit den Handelsvertretern wird deutlich, daß ein hohes Kontaktniveau die Eigenverantwortung von Handelsvertretungen für Preise und Konditionen begünstigt. Für Handelsvertreter implizieren die aufgezeigten gegenläufigen Tendenzen die Notwendigkeit einer Uberprüfung ihrer Beziehungen $z u$ den jeweiligen vertretenen Unternehmen, um im Einzelfall den eigenen Aktivitätsbereich in der Preis- und Konditionengestaltung ausbauen zu können.

Für die Erwartungen der Hersteller bei der Leistungsvergütung verdient die vielfach umstrittene Frage der Vergütung zusätzlicher Leistungen eine besondere Beachtung. Vor allem die Diskussion um eine wachsende Nachfragemacht des Handels und dem damit verbundenen Verlangen nach Serviceleistungen (z.B. Verkaufsförderung, Regalpflege) berührt unmittelbar den Tätigkeitsbereich von Handelsvertretungen und wirft an dieser stelle die Frage auf, wie die vertretenen Unternehmen die Probleme beurteilen bzw. welche Erwartungen sie an die Handelsvertretungen in diesem Bereich stellen. Im Mittelpunkt des Interesses stehen dabei weniger die geforderten Leistungen im Detail als vielmehr die Frage, wer die Kosten derartiger zusatzleistungen zu tragen hat.

Die Analyse der multiplen zusammenhänge zwischen den verschiedenen Bestimmungsfaktoren und den Erwartungen in diesem 
Aktivitätsbereich deutet eine unterschiedliche Behandlung dieser Problematik in den einzelnen Wirtschaftsbereichen an. Abbildung 34 belegt diese Zusammenhänge für alle in die Untersuchung einbezogenen Branchen.

Unterschiede in den Erwartungen hinsichtlich der Vergütung zusätzlicher Leistungen ergeben sich zwischen der Nahrungs- und Genußmittelbranche auf der einen

Seite und allen übrigen Wirtschaftsbereichen auf der anderen Seite. In den meisten Wirtschaftsbereichen erwarten die Hersteller offensichtlich einen Verzicht auf eine gesonderte Abrechnung zusätzlicher Leistungen. Während im Durchschnitt 9,6 \& aller Hersteller eine gesonderte Abrechnung erwarten, liegen beispielsweise die Werte für die Investitionsgüter- und Konsumgüterbranche mit je 6,3 \& erheblich niedriger. In diesen Wirtschaftsbereichen herrscht demnach die Meinung vor, daß sämtliche Leistungen von Handelsvertretungen mit der provision weitgehend abgegolten sind.

Einen deutlichen Wandel dieser Einstellung verzeichnet dagegen die Nahrungs- und Genußmittelbranche. Hier erwarten 16,7 \& der vertretenen Unternehmen in sehr hohem Maße eine getrennte Abrechnung von Auftrag (d.h. Provisionen) und zusätzlichen Leistungen. Noch deutlicher wird der Unterschied zu den anderen Branchen bei den Herstellern, die in etwas geringerem Ausma $B$ dieser Ansicht sind. Im Durchschnitt befürworten 15 \& die beschriebene Erwartung $z u$ einem überwiegenden Teil; der entsprechende Wert für die Nahrungs- und Genußmittelbranche liegt doppelt so hoch $(30,6$ 8) . 


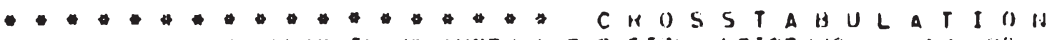

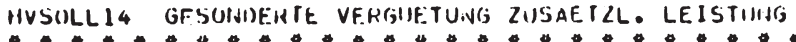

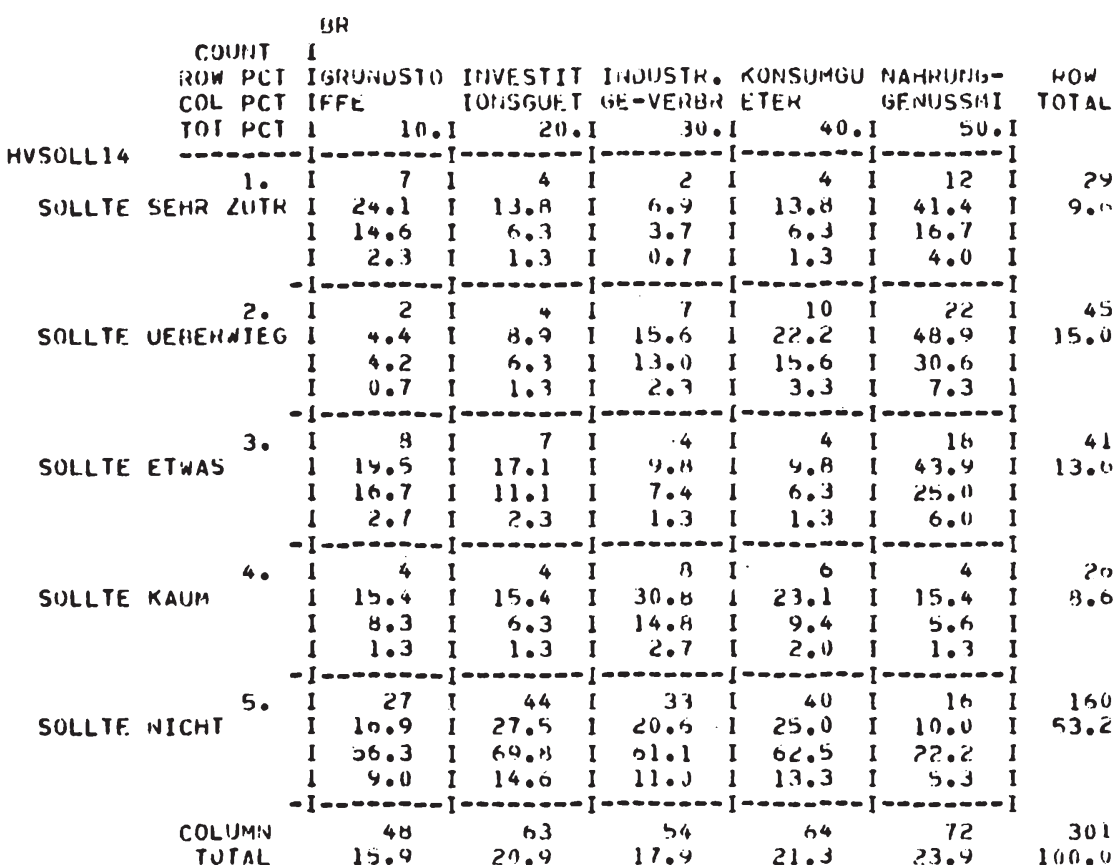

CHI SUUARE $=$ G0.2H6/3 aITH 16 I)EGKELS OF FREEDIII SIGVIFICANCE = O.0ONI

ETA $=0.37625$ WITH HVSULLIT DEPE.VUFIVT.

NUMREK OF MISSIING WISERVATIONS =

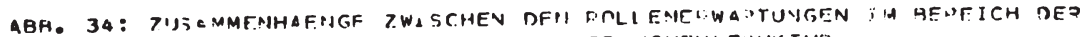
LFISTUNG SVEPGUFTUNG IINO DF= BRENCHF:ISTOUKTUS 
Die beschriebenen Resultate lassen damit Zugeständnisse der Hersteller an veränderte Marktverhältnisse in der Nahrungs- und Genußmittelbranche erkennen. Handelsvertretungen dieses Wirtschaftsbereichs sehen sich damit gegenüber anderen Branchen in einer vergleichsweise günstigeren Lage. Diese Aussage gilt jedoch nicht absolut, sondern mu $B$ im Zusammenhang mit dem generellen Provisionsgefüge der verschiedenen Branchen beurteilt werden. Beispielsweise leuchtet unmittelbar ein, daß ein hohes Ma $\beta$ an Beratungsleistungen bei Investitionsgütern Aufstellung und Inbetriebnahme von Maschinen begleiten muB - und damit Bestandteil der Handelsvertreterleistung ist - während die Preisauszeichnung von Lebensmitteln nicht ihrem unmittelbaren Aufgabenbereich angehört.

Zur Versachlichung der Diskussion um Provisionen und Zusatzleistungen sollte daher in Verhandlungen zwischen Herstellern und Handelsvertretungen ein Weg gefunden werden, die Leistungen abzugrenzen, die als Bestandteil des Provisionssatzes gelten ${ }^{1}$. Gleichzeitig sind solche Leistungsbereiche festzulegen, die eine gesonderte Vergütung bedingen; dies gilt in besonderem Maße, wenn bestimmte Leistungen nur für einzelne Kundengruppen erforderlich sind.

\subsection{Ausstattung der Handelsvertretung}

Die Beurteilung der gegenwärtigen Ausstattung von Handelsvertretungen in materieller und personeller Hinsicht sowie

1 Beispiele zur Provisionsgestaltung belegt Zarth, H.R., Setzen Sie Ihre Handelsvertreter erfolgreicher ein, in: MJ 1976, S. 438 sowie Voss, H., Spezialprobleme des Verkaufs über Handelsvertreter, in: Arbeitshandbuch Absatzförderung, Hrsg.: J. Koinecke, München 1979, S. 303 ff.; Finkenrath, R., Mehr Gewinn durch gewinnorientierte Entlohnung im Vertrieb, München 1978, S. 123 ff.. 
die Erwartungen in diesem Bereich werden in erster Linie durch allgemeine unternehmensdemographische Faktoren der vertretenen Unternehmen beeinflußt (vgl. Abbildung 26 bzw. 27) ; vertriebsbezogene Merkmale und insbesondere personale Faktoren weisen kaum eine Erhöhung der Erklärungsbeiträge auf.

Betrachtet man die als erklärungswirksam identifizierten Merkmale im Detail, so deutet Abbildung 35 Unterschiede vor allem bei Konsumgütern und Nahrungs- und Genußmitteln an. Darüber hinaus werden Handelsvertretungen in ihrer materiellen und personellen Ausstattung von umsatzstarken Herstellern tendenziell positiver eingeschätzt.

Die angesprochenen Branchenunterschiede schlüsselt Abbildung 36 weiter auf. Hinsichtlich der technischen Ausstattung erfahren durchschnittlich 16,9 \& der Handelsvertretungen eine sehr gute Beurteilung. Handelsvertretungen der Nahrungs- und Genußmittelbranche erreichen diesen Wert knapp $(16,38)$; Unternehmen der Konsumgüterbranche werden dagegen mit 14,3 \& niedriger eingestuft.

Deutlich größer werden die Abstände zwischen diesen Branchen bei Betrachtung der Befragungsschwerpunkte. $42,3 \%$ der vertretenen Unternehmen halten die gegenwärtige Ausstattung der Handelsvertretungen für überwiegend ausreichend. Bei Konsumgütern liegt dieser Wert mit nur 23,5 \& erheblich niedriger, in der Nahrungs- und Genußmittelbranche mit 55,4 \% entsprechend höher. Offensichtlich haben es die Handelsvertretungen der letztgenannten Branche besser verstanden, eine adäquate innerbetriebliche Leistungsfähigkeit sicherzustellen als Handelsvertretungen der Konsumgüterbranche. 


\begin{tabular}{|c|c|c|}
\hline ERKLÄRENDE VARIABLE & $\begin{array}{c}\text { MULTIPLE } \\
\text { KORRELATION } \\
(\mathrm{r})\end{array}$ & $\begin{array}{l}\text { BEE INFLUS- } \\
\text { SUNGSRI CHTUNG }\end{array}$ \\
\hline R_Q_L_L_E_N_E_E_B_E_Ü_L_L_U_V_L_G & & \\
\hline KONSUMGÜTER & 0,33 & + \\
\hline$B$ - HH - HB - SCHLESWIG-HOLSTEIN & 0,36 & - \\
\hline WE I TERVERARBE I TER & 0,38 & - \\
\hline UMSATZ & 0,39 & - \\
\hline NAHRUNGS- UND GENUSSMITTEL & 0.40 & - \\
\hline KG & 0.41 & + \\
\hline GMBH U, CO, KG & 0.41 & + \\
\hline$B_{-} \underline{O}_{-} L_{-} L_{-} E_{-} U_{-} E_{-} R_{-} W_{-} A_{-} B_{-} I_{-} U_{-} U_{-} G$ & & \\
\hline KONSUMGÜTER & 0.26 & + \\
\hline WE I TERVERARBE I TER & 0,30 & - \\
\hline NAHRUNGS- UND GENUSSMITTEL & 0.33 & - \\
\hline KG & 0.36 & + \\
\hline
\end{tabular}

ABB , 35: UNTERNEHMENSBEZOGENE BESTIMMUNGSFAKTOREN DER ROLLENERFÜLLUNG UND -ERWARTUNG BEI DER AUSSTATTUNG DER HANDELSVERTRETUNG 


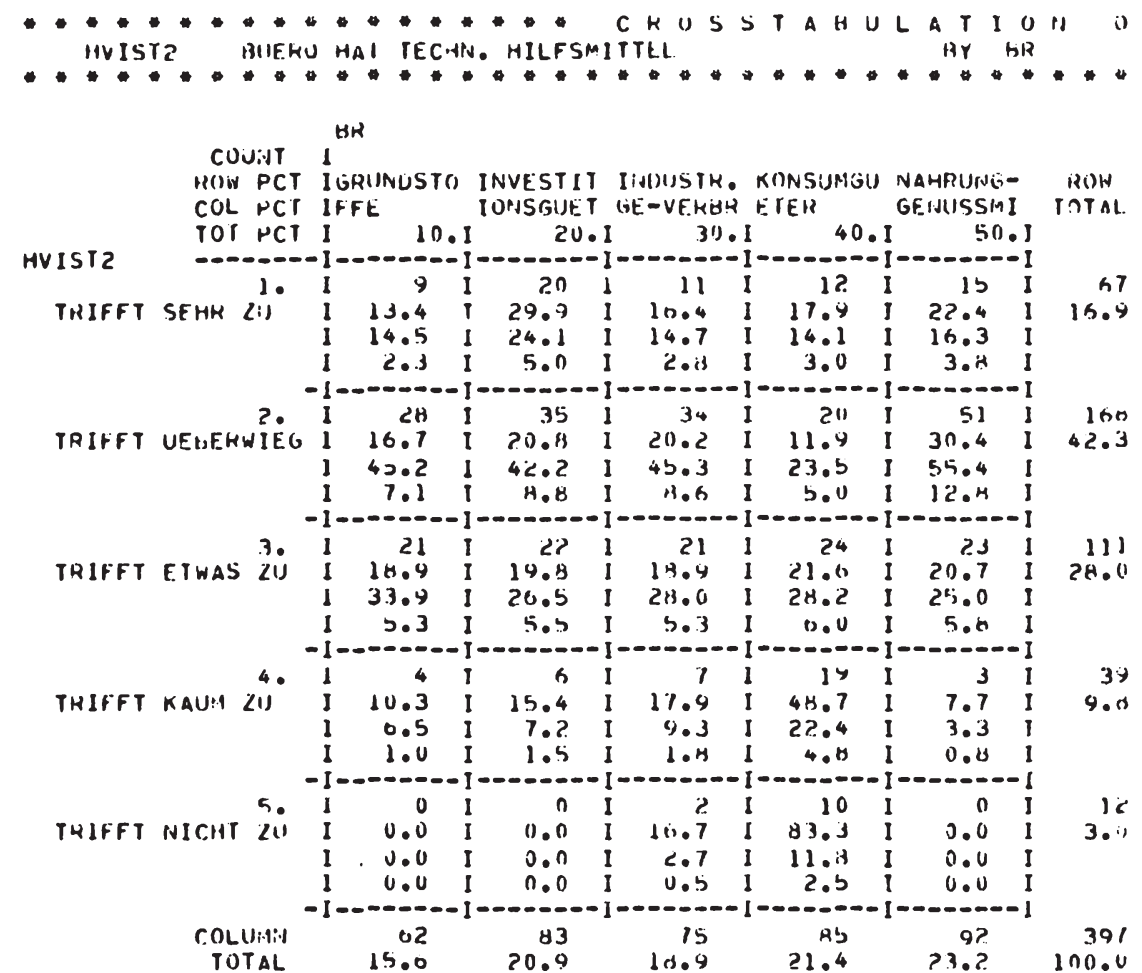

CHI SOUARE $=03.23320$ WITII 16 IJEIBRELS UF FREEUOM SI:SNIFICANCE = 10.0 ONOD ETA $=0.311538$ WITA HVIST2 DEPEIATEINT.

NUMHER OF MISSIIIS JHSERVATIUNS =

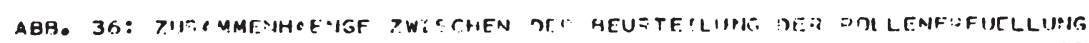

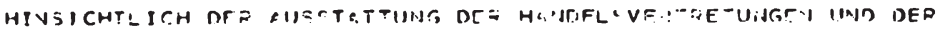
AD MRER:ATTUKTIN 
Auch das Anspruchsniveau der vertretenen Unternehmen bestätigt den hier aufgezeigten Branchenunterschied. Handelsvertretungen beider Wirtschaftsbereiche können sich durch einen Ausbau der innerbetrieblichen Leistungsfähigkeit weiter profilieren; den stärksten Anforderungen sehen sich dabei Unternehmen der Nahrungs- und Genußmittelbranche konfrontiert.

\subsection{Zusammenfassende Hypothesenbeurteilung}

Die Vielzahl der auf Herstellerebene als erklärungswirksam für eine Rollenanalyse des Handelsvertreter-Marketing abgegrenzten Bestimmungsfaktoren soll abschließend im Hinblick auf eine Beurteilung der Ausgangshypothesen zusammengefaßt werden. Abbildung 37 stellt die einzelnen Dienstleistungsbereiche von Handelsvertretungen den Resultaten der Einschätzung der Rollenerfüllung sowie den Rollenerwartungen gegenüber.

Betrachtet man zunächst die Intergruppenhypothese, so dominieren die unternehmensbezogenen Bestimmungsfaktoren - wie auf der Handelsvertreterebene - die personenbezogenen Einflußgrößen. Bei der Gestaltung von Marketingmaßnahmen müssen demnach Handelsvertretungen den bei den einzelnen Dienstleistungsbereichen vorgestellten Merkmalen der vertretenen Unternehmen besondere Aufmerksamkeit widmen.

Innerhalb der unternehmensorientierten Einflußgrößen haben sich unterschiedliche Schwerpunkte herausgestellt. Die Ergebnisse zur Intragruppenhypothese belegen für die Rollenerfüllung im Bereich der Produktanregungen eine Dominanz vertriebsbezogener Merkmale; alle anderen Leistungsbereiche werden vorwiegend von allgemeinen demographischen Merkmalen der vertretenen Unternehmen determiniert. Eine 


\begin{tabular}{|c|c|c|c|c|c|c|}
\hline \multirow[b]{3}{*}{$\begin{array}{l}\text { Dienstle } \\
\text { bereich }\end{array}$} & \multicolumn{3}{|c|}{ R० 1 le nerfullung } & \multicolumn{3}{|c|}{ Rollenerwartung } \\
\hline & $\begin{array}{c}\text { Intergruppen- } \\
\text { hypothese }\end{array}$ & $\begin{array}{l}\text { Intragruppen- } \\
\text { hypothese }\end{array}$ & $\begin{array}{c}\text { Kombinations- } \\
\text { hypothese }\end{array}$ & $\begin{array}{l}\text { Intergruppen- } \\
\text { hypothese }\end{array}$ & $\begin{array}{l}\text { Intragruppen- } \\
\text { hypothese }\end{array}$ & $\begin{array}{c}\text { Kambinations- } \\
\text { hypothese }\end{array}$ \\
\hline & $\mathrm{H}_{\text {erf1 }}^{\mathrm{H}}$ & $\mathrm{H}_{\text {erf2 }}^{\mathrm{H}}$ & $\mathrm{H}_{\text {erf3 }}^{\mathrm{H}}$ & $\mathrm{H}_{\text {erw1 }}^{\mathrm{H}}$ & $\mathrm{H}_{\text {erw2 }}^{\mathrm{H}}$ & $\mathrm{H}_{\text {erw3 }}{ }^{\mathrm{H}}$ \\
\hline Informationsverarbeitung & + & - & + & & & \\
\hline Kundenbetreuung & + & - & - & & & \\
\hline Produktanregungen & + & + & $(+)$ & & & \\
\hline $\begin{array}{l}\text { Preisgestaltung und } \\
\text { Leistungsvergütung }\end{array}$ & + & $\pm^{1)}$ & + & & & \\
\hline $\begin{array}{l}\text { Ausstattung der Handels- } \\
\text { vertretung }\end{array}$ & + & - & $(+)$ & & & \\
\hline $\begin{array}{l}\text { Information an den } \\
\text { Hersteller }\end{array}$ & + & - & + & & & \\
\hline Kundenbetreuung & & & & $\pm^{21}$ & + & + \\
\hline Informationsaustausch & & & & $\bar{t}$ & - & + \\
\hline Produktanregungen & & & & + & - & + \\
\hline Preisgestaltung & & & & + & - & + \\
\hline $\begin{array}{l}\text { Ausstattung der Handels- } \\
\text { vertretung }\end{array}$ & & & & + & - & + \\
\hline Leistungsvergütung & & & & + & + & + \\
\hline
\end{tabular}

Abb. 37: Zusammenfassende Hypothesenbeurteilung zur Rollenerfüllung und Rollenerwartung aus der Sicht der vertretenen Unternehmen

$+\quad$ : Hypothese bestätigt

- : Hypothese nicht bestätigt

(t) : Hypothese schwach bestätigt/abgelehnt
1) Erklärungsbeiträge identisch

2) jeweils keine signifikanten Erklärungsvariablen 
strategische Ausrichtung des Handelsvertreter-Marketing an den Rollenerwartungen der vertretenen Unternehmen erfordert dagegen vor allem bei der Kundenbetreuung und den Maßnahmen im Bereich der Leistungsvergütung die Berücksichtigung der als erklärungswirksam abgegrenzten vertriebsbezogenen Einflußgrößen. Wider Erwarten kann damit die Hypothese einer vorwiegenden Einflußnahme solcher Merkmale, die mit dem Vertrieb unmittelbar in Verbindung stehen abgesehen von den beschriebenen Ausnahmen - nicht aufrecht erhalten werden.

Für die Entwicklung eines Handelsvertreter-Marketing ist schließlich das Ergebnis zur Kombinationshypothese beachtenswert. Für alle Dienstleistungsbereiche konnte zwar der isolierte Einfluß personenbezogener Merkmale nicht nachgewiesen werden; die Verbindung von unternehmensorientierten und personalen Faktoren führt jedoch ebenfalls in allen Leistungsbereichen $z u$ einer besseren Erklärungsmöglichkeit der Rollenerwartungen. Bei einer daran anknüpfenden Maßnahmengestaltung sind Handelsvertretungen daher gehalten, ihr Vorgehen an den relevanten Merkmalen beider Determinantengruppen $\mathrm{zu}$ orientieren.

Eine mögliche Neukonzipierung bzw. Schwerpunktverlagerung im Dienstleistungsangebot muß darüber hinaus der zweiseitigen Einbindung von Handelsvertretungen in den Absatzkanal Rechnung tragen. Die Analyse der Beurteilung von Rollenerfüllung und Rollenerwartungen der Kunden muß daher als unabdingbarer Bestandteil in eine umfassende Rollenanalyse des Handelsvertreter-Marketing integriert werden. 
3. 3 Rollenerfüllung und -erwartung im Urteil der Abnehmer

Entsprechend dem Grundmodell einer Rollenanalyse im Handelsvertreter-Marketing ( vgl. Abbildung 9) haben die Abnehmer von Handelsvertretungen in der vorliegenden empirischen Untersuchung in gleicher Weise wie die vertretenen Unternehmen die Rollenerfüllung von Handelsvertretungen in allen Dienstleistungsbereichen beurteilt sowie ihre jeweiligen Rollenerwartungen konkretisiert ${ }^{1}$.

Die Generierung von Implikationen für das Handelsvertreter-Marketing erfordert eine Identifikation relevanter Faktoren, die einen Einfluß auf die Beurtellungen und Erwartungshaltungen ausüben. Gelingt die Abgrenzung derartiger Determinanten, lassen sich erste Anhaltspunkte für ein kunden- bzw. kundengruppenspezifisches Dienstleistungsangebot aufzeigen. Nur ein solchermaßen differenziertes Leistungsangebot kann den veränderten Marktbedingungen, die sich aus Konzentration und Kooperation auf Hersteller- und insbesondere Handelsebene ergeben ${ }^{2}$, als wirksames Aktionsprogramm gegenübergestellt werden ${ }^{3}$.

Im folgenden wird daher herausgearbeitet, welche unternehmens- und personenbezogenen Merkmale der Kontaktpartner

1 Vgl. Fragebogen 'Kunden' in Anhang II.

2 Vgl. Meyerhöfer, W., Die Handelsvermittlung - wichtiges Bindeglied in der Wirtschaft, in: ifo-Schnelldienst vom 14.5.1976, S. 24; Meffert, H., Die Handelsvertretung im Spannungsfeld des Marketing-Systems, a.a.0., S. 84 . Mit den Herstellern sind an dieser Stelle nicht die vertretenen Unternehmen sondern die Hersteller des Kundenkreises gemeint (z.B. in der weiterverarbeitenden Industrie).

3 Hierzu zählt beispielsweise ein auf die speziellen Belange von Großkunden abgestelltes Kundenmanagement; vgl. Kemna, H., Key Account Management, München 19.79, S. 24 f.; Meffert, H., Die Einführung des Kundenmanagements als Problem des geplanten organisatorischen Wandels, Arbeitspapiere des Instituts für Marketing der Universität Münster, Hrsg.: H. Meffert, Nr. 16, Münster 1979, S. 2 f.. 
von Handelsvertretungen auf der Kundenebene einen Einfluß auf Rollenerfüllung und Rollenerwartung ausüben ${ }^{1}$.

\subsection{Bestimmungsfaktoren von Rollenerfüllung und -erwar- tung im Uberblick}

Die Hypothesenprüfung über den Einfluß der Bestimmungsfaktoren auf die Rollenkomponenten erfolgte in methodischer Hinsicht analog zur Herstellerebene. Ausgehend von den ermittelten Dienstleistungsstrukturen wurden regressionsanalytisch die beschriebenen Gruppen unabhängiger Einflußgrößen auf ihren Erklärungswert hin untersucht. Für die auf Kundenebene abgegrenzten Dienstleistungsbereiche geben Abbildungen 38 und 39 die Resultate der Auswertungen im Uberblick wieder.

Die Ergebnisse bestätigen zunächst die generelle Untersuchungshypothese, daß die Beurteilung der Rollenerfüllung von Handelsvertretungen und die Rollenerwartungen auch auf Kundenebene $\mathrm{zu}$ einem erheblichen Teil durch unternehmensund personenbezogene Merkmale der befragten Abnehmerkreise erklärt werden können. Im Vergleich zu den Handelsvertretungen und Herstellern liegt das Ausmaß_der Erklärungsbeiträge mit Werten zwischen 0,44 und 0,48 für die Rollenerfüllung bzw. 0,52 und 0,59 für die Rollenerwartungen insgesamt auf einem tendenziell höheren Niveau (Spalte 5).

1 Analog zur bisherigen Vorgehensweise sind die deskriptiven Aspekte der kundenbezogenen Rollenkomponenten sowie ein Uberblick der Ausprägungen der unabhängigen Variablen in Anhang I zusammengefaßt (Tab. $6,7,8$ ). 


\begin{tabular}{|c|c|c|c|c|c|c|c|c|c|c|}
\hline \multirow[b]{2}{*}{$\begin{array}{l}\text { Dienstleistungs- } \\
\text { bereich }\end{array}$} & \multicolumn{2}{|c|}{$\begin{array}{l}\text { allgemeine } \\
\text { Unternehrens- } \\
\text { demographie } \\
\text { (1) }\end{array}$} & \multicolumn{2}{|c|}{$\begin{array}{l}\text { einkaufsbe- } \\
\text { zogene } \\
\text { Merkmale } \\
\quad(2)\end{array}$} & \multicolumn{2}{|c|}{$\begin{array}{l}\text { untemehmensbe- } \\
\text { zogene Merk- } \\
\text { male gesamt } \\
\text { (1) }+ \text { (2) } \\
\text { (3) }\end{array}$} & \multicolumn{2}{|c|}{$\begin{array}{l}\text { personenbe- } \\
\text { zogene } \\
\text { Merkmale } \\
\text { (4) }\end{array}$} & \multicolumn{2}{|c|}{$\begin{array}{l}\text { untermehmens- } \\
\text { und personenbezo- } \\
\text { gene Merkmale } \\
\text { (3) }+ \text { (4) } \\
\text { (5) }\end{array}$} \\
\hline & $\mathrm{n}_{\mathrm{v}}$ & $\frac{1}{1}$ & $n$ & $\mathbf{r}$ & $n_{v}$ & $\mathbf{r}$ & & $r$ & $n_{v}$ & $\begin{array}{ll}7 & r \\
1 & \end{array}$ \\
\hline Produktanregungen & 3 & $\left.\right|^{1} 0,23^{++}$ & 4 & $1^{1} 0,29^{++}$ & 9 & $1^{0,41^{+}}$ & 2 & $1_{1} 0,20^{+}$ & 12 & $10,47^{++}$ \\
\hline Preisgestaltung & 6 & $1^{0,35^{+1}}$ & 10 & $1^{0,34^{+t}}$ & 9 & $10,39^{+t}$ & 3 & $1^{0,21^{+}}$ & 11 & $1^{0,44^{++}}$ \\
\hline $\begin{array}{l}\text { innerbetrlebliche } \\
\text { Leistungsfähigkeit }\end{array}$ & & $1_{1}^{1} 0,28^{++}$ & 7 & $\begin{array}{l}1 \\
1\end{array}$ & 11 & $10,42^{++}$ & 3 & $\int_{1}^{1} 0,28^{++}$ & 12 & $1_{1}^{0,48^{++}}$ \\
\hline Vertretungsprogramm & & $\begin{array}{l}10,22^{+} \\
1\end{array}$ & 6 & $0,31^{++}$ & & $0,39^{+4}$ & 3 & $10,26^{++}$ & 14 & $0,45^{++}$ \\
\hline
\end{tabular}

Abb. 38: Bestimmungsfaktoren der Rollenerfüllung von Handelsvertretungen aus der Sicht der Kunden im Uberblick

$\mathrm{n}_{\mathrm{v}}$ : Anzahl der in die multiplen Regressionen einbezogenen Variablen

$r$ : multipler Korrelationskoeffizient

$\mathrm{xx}: 998-$ Signifikanzniveau

$x \quad$ : 958 - Signifikanzniveau 


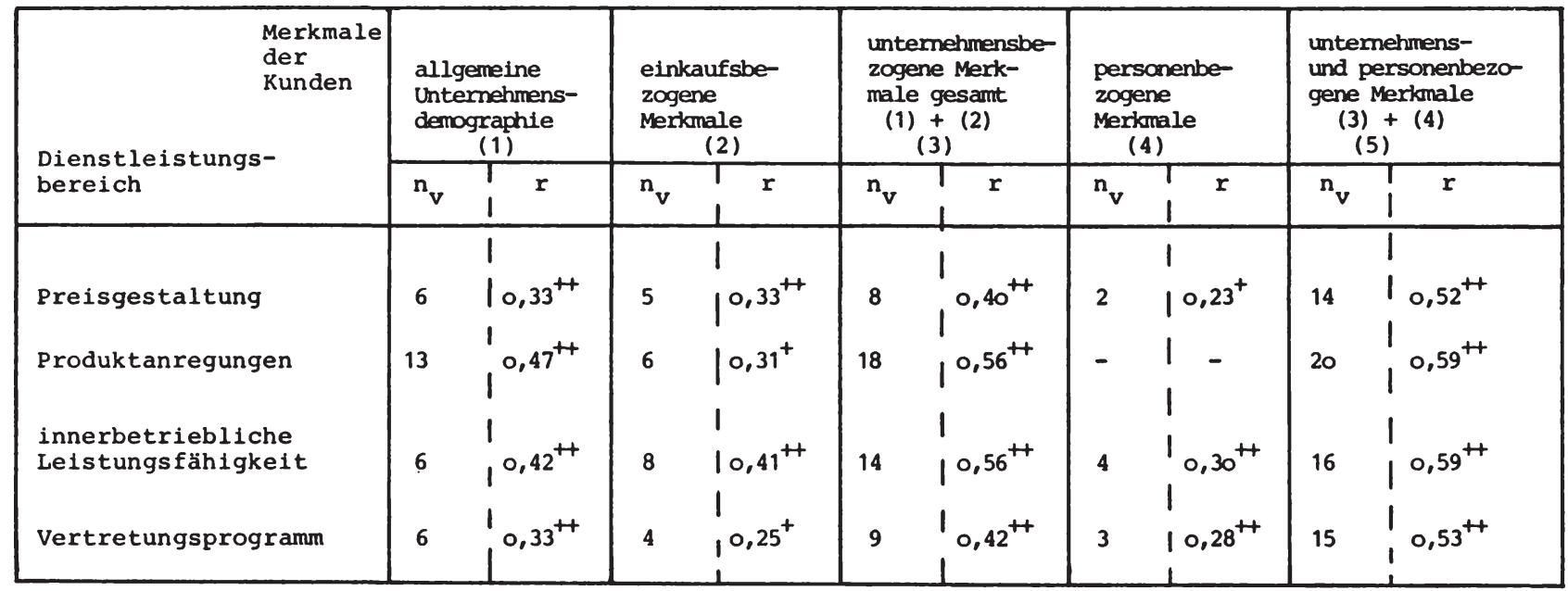

Abb. 39: Bestimmungsfaktoren der Rollererwartung an Handelsvertretungen durch die Kunden im Uberblick

$\mathrm{n}_{\mathrm{v}}$ : Anzahl der in die multiplen Regressionen einbezogenen Variablen

$r$ : multipler Korrelationskoeffizient

$+\quad: 998$ - Signifikanzniveau

$+\quad: 958$ - Signifikanzniveau 
Insbesondere die für eine strategische Ausrichtung des Handelsvertreter-Marketing wichtigen Rollenerwartungen weisen je nach Dienstleistungsbereich $27 \%$ bis $34 \%$ erklärter Varianz auf.

Der Ergebnisüberblick festigt die bei Handelsvertretungen und Herstellern bereits aufgetretene Tendenz, daß keine dominierenden Variablengruppen auftreten. Sowohl bei der Rollenerfüllung als auch bei den Rollenerwartungen führt stets eine Kombination von Merkmalsgruppen zu aussagefähigeren Ergebnissen (Spalten 3 und 5 im Vergleich zu 1,2 und 4 ).

Dieses Zwischenresultat gilt es im folgenden im Hinblick auf die spezifischen Ausprägungen in einzelnen Dienstleistungsbereichen zu differenzieren, um konkretere Ansatzpunkte einer Maßnahmenplanung aufzudecken.

\subsection{Bestimmungsfaktoren von Rollenerfüllung und -erwar- tung in einzelnen Dienstleistungsbereichen}

\subsection{Preisgestaltung}

Betrachtet man die Aktivitäten von Handelsvertretungen im Bereich der Preisgestaltung, belegen die Untersuchungsergebnisse bei globaler Betrachtung eine weitgehend gleich hohe Erklärungswirksamkeit allgemeiner Unternehmensmerkmale sowie einkaufsbezogener Merkmale. Dies gilt sowohl für die Beurteilung preis- und konditionenpolitischer Handlungsspielräume bei der Rollenerfüllung als auch bei den Rollenerwartungen der Kunden. Weiterhin führt die Einbeziehung personaler Merkmale der Kontaktpartner von Handelsvertretern bei ihren Kunden zu einer teilweise erheblichen Verbesserung der Erklärungsmöglichkeiten. Für die Rollener- 
füllung steigt der Erklärungsbeitrag um $15 \%$ (von 0,41 auf 0,47 ), für die Rollenerwartungen sogar um $30 \%$ (von 0,40 auf 0,52$)$.

Eine detaillierte Analyse der Rollenerfüllung ermöglicht weitergehende Aussagen vor allem für die Handelsvertretungen, die in der Investitionsgüterbranche Erzeugnisse an tendenziell größere Abnehmer verkaufen (vgl. Abbildung 40). Hier wird der preis- und konditionenpolitische Handlungsspielraum der Handelsvertreter als relativ eng empfunden, wenn die Kunden zugleich einen hohen Anteil ihres übrigen Einkaufs mit (anderen) Lieferanten direkt abwickeln.

Hinsichtlich der Verhandlungsdurchführung bestätigt die Untersuchung diese Tendenz vor allem für Verkaufsgespräche auf Geschäftsführungsebene, wobei das Kaufverhalten der beschriebenen Kundengruppe durch einen kollektiven Entscheidungsproze $\beta$ determiniert wird. Eine auffallend niedrige Einschätzung des Handlungsspielraumes von Handelsvertretungen weisen dabei jene Verhandlungspartner auf, die ihre Position im Unternehmen bereits relativ lange innehaben. Auch führt ein verstärkter Anteil telefonischer Auftragsabwicklungen nicht $z u$ einer Verbesserung der Beurteilung des preispolitischen Freiraumes von Handelsvertretungen.

Es ist anzunehmen, daß die für die Investitionsgüterbranche aufgezeigten Resultate in erster Linie für hier überwiegend anzutreffenden industriellen Abnehmergruppen gelten. Zur Beschreibung der Beurteilung des preispolitischen Aktivitätsraumes von Handelsvertretungen anderer Wirtschaftsbereiche wurde ergänzend eine Differenzierung der Beurteilungen nach den unterschiedlichen Betriebsformen der 


\begin{tabular}{|c|c|c|}
\hline ERKLÄRENDE VARIABLE & $\begin{array}{c}\text { MULTIPLE } \\
\text { KORRELATION } \\
(r) \\
\end{array}$ & $\begin{array}{l}\text { BEEE INFLUS- } \\
\text { ŜNGSR I CHTUNG }\end{array}$ \\
\hline RECHTSFORM AG & 0,17 & + \\
\hline INVESTITIONSGÜTER & 0.24 & - \\
\hline EINKAUFSANTEIL VON HERSTELLERN & 0.29 & + \\
\hline$B$ - HH - HB - SCHLESWIG-HOLSTEIN & 0.33 & - \\
\hline DAUER DER AUSGEÜBTEN TÄTIGKEIT & 0.35 & + \\
\hline UMSATZ & 0.37 & + \\
\hline $\begin{array}{l}\text { AUFTRAGSABWICKLUNG MIT TELEFON/ } \\
\text { TELEXX }\end{array}$ & 0.38 & + \\
\hline BUNDESWEITES ANGEBOT & 0.40 & + \\
\hline $\begin{array}{l}\text { KAUFENTSCHEIDUNG DURCH MEHRERE } \\
\text { PERSONEN }\end{array}$ & 0.42 & - \\
\hline GESCHÄFTSFÜHRER & 0.43 & - \\
\hline GESAMTES SORTIMENT VON HV & 0,44 & - \\
\hline
\end{tabular}

ABB, 40: UNTERNEHMENS- UND PERSONENBEZOGENE BESTIMMUNGSFAKTOREN DER ROLLENERFÜLLÜNG VON HANDELSVERTRETUNGEN BEI DER PREISGESTALTUNG 
Abnehmerseite ermittelt (vgl. Abbildung 41) ${ }^{1}$.

Unabhängig von der Unterscheidung einzelner Branchen bestätigt die Ubersicht die für die Investitionsgüterbranche ausgesprochene Vermutung, d.h. von den industriellen Abnehmern wird der preispolitische Handlungsspielraum der Handelsvertretungen tendenziell eng eingeschätzt. Die entsprechenden Werte für diesen Abnehmerkreis bewegen sich - im Vergleich $\mathrm{zu}$ anderen Betriebsformen - in Höhe des Durchschnittswertes aller Befragten. Eine ebenfalls durchschnittliche Beurteilung weist der Fachgroßhandel auf.

Deutliche Abweichungen kennzeichnen die Situation bei den Einkaufsverbänden und dem Facheinzelhandel. Während durchschnittlich 238 der Kunden einen hinreichenden Handlungsspielraum der Handelsvertreter verneinen, liegt dieser Wert mit über 26 \& für die Einkaufsverbände erheblich höher. Demgegenüber halten $35 \&$ der befragten Einzelhandelsbetriebe den Handlungsspielraum für ausreichend, lediglich 23 im Durchschnitt aller Kunden sind dieser Meinung.

Diese differenzierte Beurteilung der Handelsvertretungen spiegelt die unterschiedlichen Interessen der Kundengruppen wider. Offenbar legen größere Kunden - wie z.B. Einkaufszentralen - ein höheres und alleiniges Gewicht auf den Preis im Rahmen der Einkaufsverhandlungen, während der Preis im Einzelhandel eher im Verbund mit anderen Leistungen der Handelsvertretung gesehen wird, die für Großabnehmer

1 Die Abbildung weist zuvor signifikante Zusammenhänge aus (95 8-Niveau); die Befunde können demnach nur als Tendenzaussagen formuliert werden, weil einige der zellen nicht die für den Chi-Quadrat-Test erforderliche Klassenhäufigkeit aufweisen; vgl. hierzu Bleymüller, J., Gehlert, G., a.a.O., S. 317 ff.; Yamane, T., a.a.O., S. 648 ff.. 


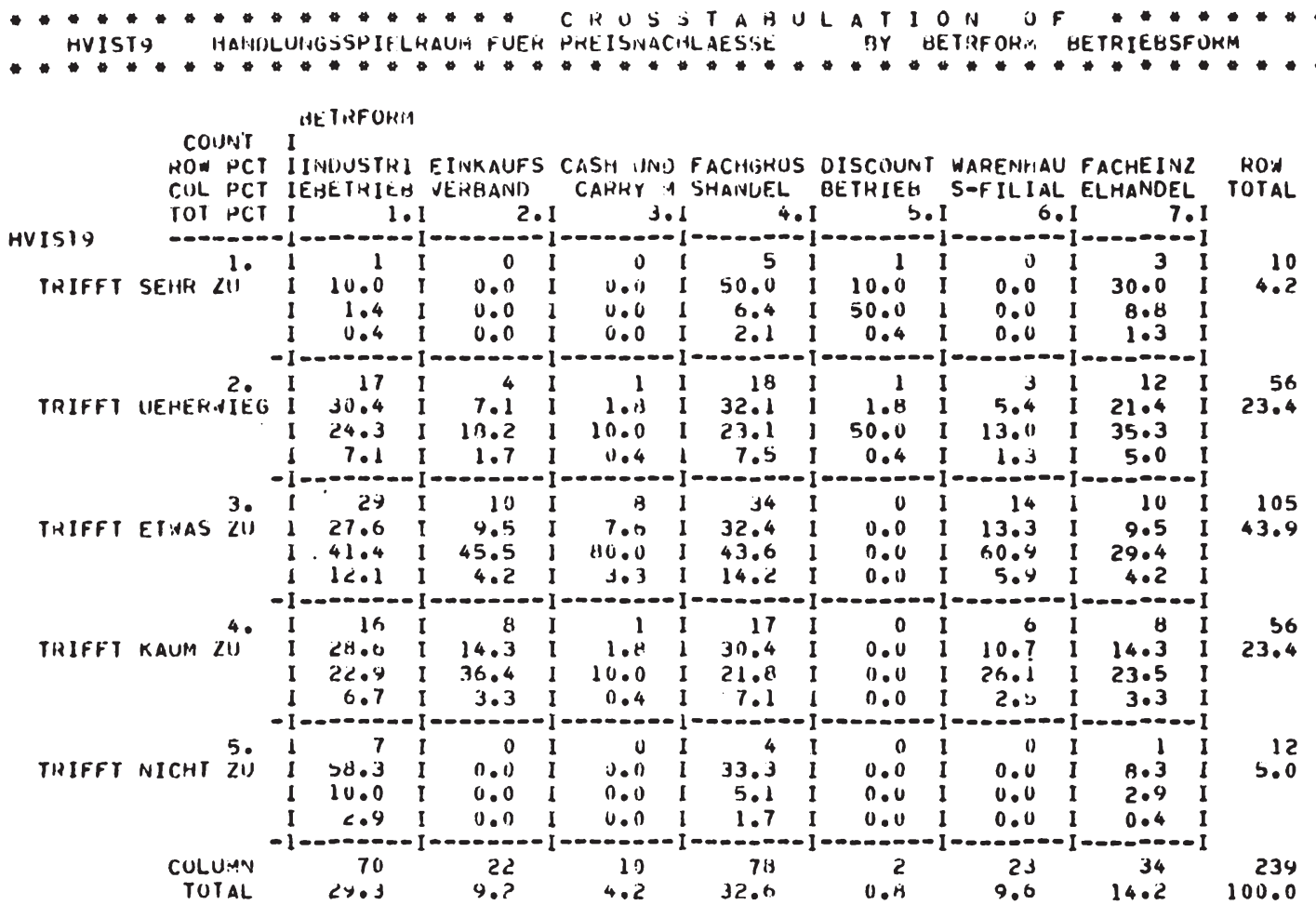

CHI SUUAMF $=\quad 37.71336$ WITH 24 UE UKEES UF FHEEDOM SIGNIFICANCE = 0.0371

ETA $=0.21542$ WIII IVISIY DEPEIIIEAT. = 0.16334 WITH BETRFOHM DEPENUENT. IUUMBEH IOF MISSIMG VISERVATIWIS $=$

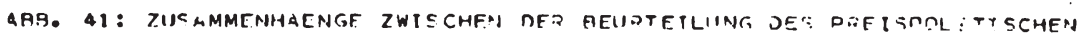
HOA!DLUNGSSDIFL = IIME UND UNTFESCH!EDL CIEN RFTDIEHST T=MEV ALF KUNOFPEBEAL 
(Einkaufsverbände, -zentralen) weniger bedeutsam sind (z.B. allgemeine Brancheninformationen, Sortimentsanregungen).

Als wesentliche Konsequenz dieser Resultate ist festzuhalten, daß Handelsvertretungen im Rahmen ihrer Maßnahmenplanung stets die Wirkung ihrer Aktivitäten antizipieren müssen. Das Beispiel aus der Preisgestaltung deckt hierfür eine unterschiedliche Marktreaktion bei gleichem Einsatz von Aktivitäten auf; d.h. ein gleicher Handlungsspielraum für Preisverhandlungen führt $z u$ abweichenden Beurteilungen bei den verschiedenen Kundengruppen. Neben der kritisch zu überprüfenden Forderung der Kunden nach generell höheren Handlungsspielräumen sollten Handelsvertreter vielmehr versuchen, bei ihren vertretenen Unternehmen eine Differenzierung ihrer Preisverantwortung bei unterschiedlichen Kundengruppen zu erwirken, um auf diese Weise unter Vermeidung verzögernder Rückfragen die Flexibilität am Markt zu erhöhen.

Die Analyse der Rollenerwartungen an Handelsvertretungen im Bereich preispolitischer Aktivitäten läßt - im Vergleich zur Beurteilung der Rollenerfüllung - keine prinzipiell abweichenden Resultate erkennen. Hervorzuheben ist lediglich der bereits beim Gesamtüberblick erwähnte Erklärungszuwachs unternehmensorientierter Merkmale durch die Einbeziehung personaler Faktoren.

\subsection{Produktanregungen}

Die Aktivitäten von Handelsvertretungen bei der Mitwirkung der Produkt- und Sortimentsentwicklungen ihrer vertretenen Unternehmen umfassen aus der Sicht der Kunden eine Reihe 
heterogener Leistungsmerkmale. Bei der Strukturierung der Dienstleistungsbereiche von Handelsvertretungen haben die Kunden neben den Anregungen zur Entwicklung neuer Produkte sowie der Einflußnahme auf das Herstellersortiment auch informationsbezogene Aktivitäten diesem Dienstleistungsbereich zugeordnet (vgl. Abbildung 15 bzw. 16). Insbesondere die Verwertung von Kundeninformationen bei der Einflußnahme auf den Hersteller stellt aus der Sicht der Abnehmer eine zentrale Aufgabe in diesem Bereich dar.

Die Analyse der Bestimmungsfaktoren der gegenwärtigen Beurteilung von Handelsvertretungen im Bereich der Produktanregungen läßt aus Kundensicht deutliche Schwächen der Handelsvertreter erkennen. Die Beurteilung dieser Schwachstellen hängt jedoch $z u$ einem erheblichen Maße von der Einkaufsstruktur der betrachteten Abnehmer ab (vgl. Abbildung 42). Diejenigen Kunden, die - neben dem Einkauf über Handelsvertreter - einen hohen Anteil ihrer wertmäBigen Bezüge mit Herstellern direkt oder mit Reisenden abwickeln, sehen die Leistungen der Handelsvertreter bei den Produktanregungen tendenziell negativ. Sinkt dagegen der wertmäßige Einkaufsanteil von Reisenden und Herstellern, werden die Aktivitäten der Handelsvertreter eher positiv eingeschätzt. Offensichtlich sehen solche Kunden, die nur relativ wenig direkte Kontakte zu Herstellern haben, ihre Interessen durch die Handelsvertreter in Bezug auf die Produkt- und Sortimentsgestaltung der Hersteller bereits in hinreichendem Maße vertreten.

Eine Chance zur Profilierung für Handelsvertretungen zeigen dagegen die Beurteilungen der erstgenannten Kundengruppe an. Abnehmer, die ihrerseits relativ viele Kontakte mit Herstellern haben, schätzen die Leistungen der Handelsvertretungen eher kritisch ein. Es kann vermutet werden, 
daß die Kunden aus ihren unmittelbaren Herstellerbeziehungen - und den dabei möglicherweise deutlich werdenden produkt- und sortimentspolitischen Schwächen - ein mangelndes Engagement von Handelsvertretungen zur Einwirkung auf das Produktangebot in ihrem Sinne ableiten. So ist beispielsweise anzunehmen, daß die Kunden bei einem wenig auf ihre Bedürfnisse abgestimmten Produktangebot den Handelsvertretungen eine nicht befriedigende Verwertung von (Kunden-) Informationen anlasten.

Zieht man zur tberprüfung dieser Vermutung die an die Rolle der Handelsvertretungen geknüpften Erwartungen zusätzlich heran, ist eine Relativierung der diskutierten Zusammenhänge angezeigt (vgl. Abbildung 42). Die Resultate erweisen sich dabei als ambivalent.

Die in Bezug auf die Einkaufsstruktur der Kunden formulierte Vermutung kann nur in abgeschwächter Form aufrecht erhalten werden, da die Kunden mit hohem Einkaufsanteil durch Direktbezug vom Hersteller ein niedrigeres Erwartungsniveau hinsichtlich der Aktivitäten von Handelsvertretungen bei den Produktanregungen aufweisen. Eine vollständige Ablehnung der beschriebenen Zusammenhänge ist dagegen nicht möglich, weil die Ergebnisse der Studie belegen, daß eine hohe $\mathrm{Zahl}$ von Einkäufern - die erfahrungsgemäB bei größeren Abnehmern und umfangreicherem Herstellerdirektbezug auftreten - mit einer hohen Erwartungshaltung der Kunden an die Handelsvertretungen zur Mitwirkung beim Produktprogramm der Hersteller verbunden ist.

In jedem Falle eröffnen die diskutierten Resultate zu den Kundenbeurteilungen für Handelsvertretungen die Möglichkeit einer Leistungsverbesserung gegenüber ihren Abnehmern, wenn es ihnen gelingt, den Kunden eine wirksame Einflußnahme auf das Angebotsprogramm der Hersteller glaubhaft zu 


\begin{tabular}{|c|c|c|}
\hline ERKLÄRENDE VARIABLE & $\begin{array}{c}\text { MULTIPLE } \\
\text { KORRELATION } \\
(r)\end{array}$ & $\begin{array}{l}\text { BEE INFLUS- } \\
\text { SUNGSR I CHTUNG }\end{array}$ \\
\hline B_O_L_L_E_N_E_E_R_E_Ü_L_L_U_N_G & & \\
\hline EINKAUFSANTEIL VON HERSTELLERN & 0.20 & + \\
\hline $\begin{array}{l}\text { WESENTLICHE SORTIMENTSANTEILE } \\
\text { VON REISENDEN }\end{array}$ & 0.25 & + \\
\hline $\begin{array}{l}\text { KAUFENTSCHEIDUNG DURCH MEHRERE } \\
\text { PERSONEN }\end{array}$ & 0.28 & - \\
\hline EINKAUFSANTEIL VON REISENDEN & 0,29 & + \\
\hline B_Q_L_L_E_E_W_E_R_W_E_R_R_I_U_U_G & & \\
\hline EINKAUFSANTEIL VON REISENDEN & 0.15 & + \\
\hline $\begin{array}{l}\text { AUFTRAGSABWICKLUNG MIT TELEFON/ } \\
\text { TELEX }\end{array}$ & 0.21 & + \\
\hline $\begin{array}{l}\text { WESENTLICHE SORTIMENTSANTEILE } \\
\text { VON HANDELSVERTRETERN }\end{array}$ & 0.25 & - \\
\hline ZAHL DER EINKÄUFER & 0.27 & - \\
\hline EINKAUFSANTEIL VON HERSTELLERN & 0.29 & + \\
\hline $\begin{array}{l}\text { ERGÄNZENDE SORTIMENTSANTE ILE } \\
\text { VON HANDELSVERTRETERN }\end{array}$ & 0.31 & + \\
\hline
\end{tabular}

ABB， 42: EINKAUfSBEZOGENE BESTIMMUNGSFAKTOREN DER ROLLENERFÜLLUNG UND -ERWARTUNG DER KUNDEN BEI DEN PRODUKTANREGUNGEN 
machen. Die Position der Handelsvertreter als selbständige Vermittler zwischen Industrie und Handel kann dadurch nachhaltig gefestigt werden.

\subsection{Innerbetriebliche Leistungsfähigkeit}

Die innerbetriebliche Leistungsfähigkeit der Handelsvertretungen wird von den Kunden in globaler Hinsicht relativ positiv beurteilt ${ }^{1}$. Die Frage nach den Bestimmungsfaktoren dieser Beurteilung führt auf einen dominierenden EinfluB einkaufsbezogener Merkmale der Kunden. Kriterien der Einkaufsorganisation bzw. Auftragsabwicklung weisen bei der Einschätzung der Rollenerfüllung wesentlich höhere Erklärungsbeiträge auf als allgemeine unternehmensdemographische Faktoren. Bei den Erwartungshaltungen der Abnehmer nähern sich Erklärungswerte beider Variablengruppen einander an (vgl. Abbildung 38 und 39).

Betrachtet man eingehender die gegenwärtige Einschätzung der materiellen und personellen Ausstattung von Handelsvertretungen durch ihre Abnehmer, lassen sich in erster Linie Kriterien der Bezugsquellenstruktur als Prädiktoren heranziehen. Ein besonderes Gewicht kommt der innerbetrieblichen Leistungsfähigkeit nach den Resultaten von Abbildung 43 bei solchen Kunden $z u$, die den größten Teil ihres Sortiments über Reisende beziehen, während von Handelsvertretern eher sortimentsergänzende produkte gekauft werden.

Diese Kunden haben den eigenen Einkauf vorwiegend nach dem eigenen Sortiment organisatorisch aufgebaut. Die Auftragsabwicklung erfolgt in hohem Maße weniger durch persönliche Kontakte mit den Anbietern (d.h. Handelsvertretern und

1 Vgl. Tab. 6, Anhang I. 
Reisenden), als vielmehr durch telefonische oder fernschriftliche Kontakte. Je höher der Anteil dieser unpersönlichen Beziehungen ausfällt, um so positiver wird in der Tendenz die innerbetriebliche Leistungsfähigkeit der Handelsvertretungen angesehen. Gleichzeitig ist jedoch im Hinblick auf mögliche Schlußfolgerungen zu beachten, daß die positive Einschätzung abnimmt, wenn ein Kunde einen höheren Teil seines Einkaufsvolumens mit Handelsvertretern abwickelt.

Die Kenntnis, welcher Anteil des gesamten Einkaufs eines Kunden über Handelsvertreter insgesamt abgewickelt wird, versetzt eine einzelne Handelsvertretung demnach in die Lage, ein auf die Bedürfnisse unterschiedlicher Kundengruppen abgestimmtes Niveau der innerbetrieblichen Leistungsfähigkeit einzurichten. Ob eine bestimmte Leistung notwendig ist - beispielsweise eine ganztägige Besetzung des Büros hängt dabei nicht allein von der quantitativen Größe der abgegrenzten Gruppen mit unterschiedlichem Leistungsbedarf $a b$. Die Modifizierung bzw. Neuaufnahme von Leistungen kann vielmehr nur in unmittelbarer Verbindung mit den Umsatzbeiträgen der einzelnen Kundengruppen entschieden werden. Hierbei ist eine Abwägung der zusätzlich entstehenden Kosten mit den möglicherweise drohenden Kunden- bzw. Auftragsverlusten unerläßlich.

Die bereits aus der Einschätzung der Rollenerfüllung ableitbaren Konsequenzen erfahren durch die Einbeziehung der Rollenerwartungen an Handelsvertretungen im Bereich der innerbetrieblichen Leistungsfähigkeit eine Bestätigung (vgl. Abbildung 43). Die Bezugsquellenstruktur erweist sich auch bei den Erwartungen der Kunden als dominante Einflußgröße. Abnehmergruppen, die ihre Einkaufsumsätze zu großen Teilen mit Herstellern bzw. Reisenden abwickeln und entsprechend niedrigeren Anteilen mit Handelsvertretungen - stellen an die innerbetriebliche Leistungsfähigkeit der Handelsvertretungen besonders hohe Anforderungen. 


\begin{tabular}{|c|c|c|}
\hline ERKLÄRENDE VARIABLE & $\begin{array}{c}\text { MULTIPLE } \\
\text { KORRELATION } \\
(r)\end{array}$ & $\begin{array}{l}\text { BEE INFLUSSUNGS- } \\
\text { RICHTUNG }\end{array}$ \\
\hline B_Q_L_L_E_N_E_E_B_E_U___L_L_U_N_G & & \\
\hline $\begin{array}{l}\text { AUFGABENABWICKLUNG MIT TELEFON/ } \\
\text { TELEX }\end{array}$ & 0,19 & - \\
\hline $\begin{array}{l}\text { EINKAUF SORGANISATION NACH EIGENEM } \\
\text { SORTIMENT }\end{array}$ & 0.24 & + \\
\hline GESAMTES SORTIMENT VON REISENDEN & 0.31 & - \\
\hline $\begin{array}{l}\text { ERGÄNZENDE SORTIMENTSTEILE VON } \\
\mathrm{HV}\end{array}$ & 0.33 & + \\
\hline EINKAUFSANTEIL VON HV & 0.34 & + \\
\hline$B_{-} Q_{-} L_{-} L_{-} E_{-} V_{-} E_{-} R_{-} W_{-} A_{-} B_{-} I_{-} U_{-} \mathbb{N}_{-} G$ & & \\
\hline $\begin{array}{l}\text { AUFTRAGSABWICKLUNG MIT TELEFON/ } \\
\text { TELEX }\end{array}$ & 0,20 & - \\
\hline $\begin{array}{l}\text { EINKAUFSORGANISATION NACH EIGE- } \\
\text { NEM SORTIMENT }\end{array}$ & 0,27 & + \\
\hline EINKAUFSANTEIL VON HERSTELLERN & 0.32 & - \\
\hline $\begin{array}{l}\text { KAUFENTSCHEIDUNG DURCH EINKÄU- } \\
\text { FER ALLEIN }\end{array}$ & 0,35 & - \\
\hline $\begin{array}{l}\text { ERGÄNZENDE SORTIMENTSANTEILE VON } \\
\mathrm{HV}\end{array}$ & 0.37 & + \\
\hline EINKAUFSANTEIL VON REISENDEN & 0.39 & - \\
\hline EINKAUFSANTEIL VON HV & 0.40 & + \\
\hline $\begin{array}{l}\text { ERGÄNZENDE SORTIMENTSANTEILE } \\
\text { VON RE I SENDEN }\end{array}$ & 0,41 & - \\
\hline
\end{tabular}

ABB, 43: EINKAUFSBEZOGENE BESTIMMUNGSFAKTOREN DER ROLLENERFÜLLUNG UND -ERWARTUNG DER KUNDEN BEI DER INNERBETRIEBLICHEN LEISTUNGSFÄHIGKEIT 
Die beschriebenen Auswirkungen der Einschätzung von Rollenerfüllung sowie Rollenerwartungen lassen zusammenfassend eine Chance zur Profilierung von Handelsvertretungen gegenüber herstellerdominierten Vertriebswegen erkennen. Eine Veränderung des Leistungsangebotes darf dabei nicht $z u$ isolierten Einzelaktionen führen, sondern muß im gesamten Leistungsangebot abgestimmt erfolgen.

\subsection{Vertretungsprogramm}

Bei der Beurteilung des Vertretungsprogramms haben die Kunden ihre Einschätzungen darüber zum Ausdruck gebracht, inwieweit es den Handelsvertretungen gelungen ist, die verschiedenen Vertretungen so auszuwählen, daß ein insgesamt kundengerechtes Angebot zustande gekommen ist; weiterhin wurden die Erwartungen für diesen Leistungsbereich präzisiert.

Unterschiede in den Bestimmungsfaktoren der Einschätzung von Rollenerfüllung und -erwartung belegt bereits der Ergebnisüberblick (vgl. Abbildung 38 bzw. 39). Demnach läßt sich die Rollenerfüllung $z u$ erheblich größeren Teilen durch einkaufsbezogene Merkmale der Abnehmer erklären; die Rollenerwartungen weisen insgesamt höhere Erklärungsbeiträge der allgemeinen unternehmensdemographischen Merkmale auf. Die Aussagefähigkeit der letztgenannten Faktoren ist trotz ihres quantitativen Ausmaßes für das Handelsvertreter-Marketing von untergeordneter Bedeutung, weil sich lediglich heterogene Faktoren der regionalen Ansiedlung bzw. Rechtsform als erklärungswirksam abgrenzen lassen. Aus diesem Grunde werden die einkaufsbezogenen Merkmale vorrangig eingehender analysiert. 
Bei Rollenerfüllung und Rollenerwartung belegen die Resultate der Studie hohe Erklärungswerte solcher Variablen, die die Struktur der Bezugsquellen und das Kaufentscheidungsverhalten der Kunden beschreiben (vgl. Abbildung 44). Die Ergebnisse zur Einschätzung der gegenwärtigen Leistungserfüllung bei der Gestaltung ihres Vertretungsprogramms unterteilen die Kunden tendenziell in zwei Gruppen.

\begin{tabular}{|c|c|c|}
\hline ERKLÄRENDE VARIABLE & $\begin{array}{l}\text { MULTIPLE } \\
\text { KORRELATION }\end{array}$ & $\begin{array}{l}\text { BEE I NFLUS- } \\
\text { SUNGSR I CHTUNG }\end{array}$ \\
\hline EINKAUFSANTEIL VON HERSTELLERN & 0.16 & + \\
\hline $\begin{array}{l}\text { KAUFENTSCHEIDUNG DURCH MEHRERE } \\
\text { PERSONEN }\end{array}$ & 0.23 & - \\
\hline $\begin{array}{l}\text { ERGÄNZENDE SORTIMENTSTEILE VON } \\
\text { REISENDEN }\end{array}$ & 0.26 & - \\
\hline $\begin{array}{l}\text { AUFTRAGSABWICKLUNG MIT TELEFON/ } \\
\text { TELEX }\end{array}$ & 0.28 & - \\
\hline $\begin{array}{l}\text { WESENTLICHE SORTIMENTSTEILE VON } \\
\text { HV }\end{array}$ & 0.29 & + \\
\hline $\begin{array}{l}\text { KAUFENTSCHEIDUNG DURCH EINKÄUFER } \\
\text { ALLEIN }\end{array}$ & 0,31 & - \\
\hline
\end{tabular}

ABB, 44: EINKAUfSBEZOGENE BESTIMMUNGSFAKTOREN DER ROLLENERFÜLLUNG AUS KUNDENSICHT BEIM VERTRETUNGSPROGRAMM

Die erste Gruppe umfaßt solche Kunden, deren Kaufentscheidungen kollektiv getroffen werden. Da ein derartiges Kaufverhalten vornehmlich bei größeren Unternehmen bzw. Einkaufsverbänden anzutreffen ist, die erfahrungsgemä $\beta$ 
einen großen Teil ihrer Einkäufe mit Herstellern direkt abwickeln, verwundert es kaum, daß die Handelsvertretungen mit ihrem Vertretungsprogramm von diesen Kunden eher negativ eingeschätzt werden. Es muß an dieser stelle die Frage unbeantwortet bleiben, ob die beschriebene Kundengruppe ihre niedrige Beurteilung ausschlieblich auf das jeweilige Angebotsprogramm der Handelsvertretungen projiziert, oder ob sie dem Einkauf über Handelsvertreter - angesichts des hohen Ausmaßes direkter Herstellerbeziehungen - grundsätzlich skeptischer gegenübersteht ${ }^{1}$.

Die erklärungswirksamen Bestimmungsfaktoren beinhalten weiterhin eine Gruppe von Kunden, deren Kaufverhalten durch Einpersonenentscheidungen gekennzeichnet ist. Bei den Unternehmen, in denen Einkäufer allein über die Auftragsvergabe entscheiden, tragen die Handelsvertretungen tendenziell in größerem Umfang zum Gesamtsortiment der Kunden bei, d.h. sie verkaufen an die Kunden wesentliche Sortimentsbestandteile. Die dadurch notwendig werdenden häufigeren Kontakte zwischen Handelsvertretung und Kunde werden vornehmlich telefonisch bzw. fernschriftlich abgewickelt. Unternehmen dieser Gruppe beurteilen das Vertretungsprogramm nach vorliegenden Resultaten insgesamt positiver.

Die Polarisierung der Kundenbeurteilungen über das Vertretungsprogramm von Handelsvertretungen setzt sich bei den Erwartungshaltungen der Kunden nicht fort. Die studie belegt hohe Anforderungen an Handelsvertretungen hinsichtlich der Gestaltung des Vertretungsprogramms vor allem bei umsatzstarken Kunden mit hohem Einkaufsanteil von Herstellern direkt sowie weitgehend kollektivem Kaufverhalten (vgl.Abb.45).

1 Speziell bei der Einführung neuer Produkte sehen sich Handelsvertretungen bei Kaufentscheidungen durch Gremien Problemen ausgesetzt; vgl. die empirischen Befunde von Dichtl, E., Bauer, H.H., Die Hersteller-Handels-Kommunikation bei neuen Produkten auf dem Prüfstand, Nr. 6 der Information zur Rationalisierung der Kommunikation, Frankfurt 1978 . 


\begin{tabular}{|l|c|c|}
\hline \multicolumn{1}{|c|}{ ERKLÄRENDE VARIABLE } & $\begin{array}{c}\text { MULTIPLE } \\
\text { KORRELATION } \\
(\mathrm{r})\end{array}$ & $\begin{array}{c}\text { BEE INFLUSSUNGS- } \\
\text { RICHTUNG }\end{array}$ \\
\hline B - HH - HB - SCHLESWIG-HOLSTEIN & 0.17 & - \\
$\begin{array}{l}\text { KAUFENTSCHE IDUNG DURCH MEHRERE } \\
\text { PERSONEN }\end{array}$ & 0.23 & - \\
ZAHL DER WARENGRUPPEN & 0.28 & + \\
EINZELUNTERNEHMUNG & 0.31 & + \\
EINKAUFSANTE IL VON HERSTELLERN & 0.34 & - \\
UMSATZ & 0.37 & + \\
$\begin{array}{l}\text { KAUFENTSCHEIDUNG DURCH } \\
\text { GESCHÄFTSFÜHRER }\end{array}$ & 0.39 & - \\
EINKAUFSANTEIL ÜBER ZENTRALE & 0.41 & + \\
GRUNDSTOFFE & 0.42 & + \\
\hline
\end{tabular}

AbB . 45: UNTERNEHMENSBEZOgEnE BESTIMMUNGSFAKTOREN DER ROLLENERWARTUNG AUS KUNDENSICHT BEIM VERTRETUNGSPROGRAMM 
Die bei der Beurteilung der Rollenerfüllung hervorgetretene Gruppe mit Einpersonenentscheidungen läßt sich bei den Erwartungen der Kunden an die Rolle der Handelsvertretungen nicht abgrenzen.

Für die Maßnahmenplanung im Handelsvertreter-Marketing beinhalten diese Resultate eine zweiseitige Konseguenz. Zum einen müssen Handelsvertretungen bei solchen Kunden, deren Einkäufer vorwiegend allein entscheiden und bei denen sie wesentliche Teile zum Kundensortiment beitragen, dafür Sorge tragen, die relativ positiven Einschätzungen hinsichtlich ihres Vertretungsprogramms beizubehalten bzw. auszubauen. Offensichtlich ist es Handelsvertretungen bereits gelungen, in dem beschriebenen Kundensegment ihre Position durch eine kundengerechte Zusammenstellung der Vertretungen zu festigen.

Schwieriger ist die Position der Handelsvertretungen dagegen bei größeren Kunden mit kollektivem Kaufentscheidungsverhalten. Hier ist den hohen Anforderungen dieser Kundengruppe an das Vertretungsprogramm besondere Aufmerksamkeit $\mathrm{zu}$ widmen und damit eine mögliche Realisierbarkeit $\mathrm{zu}$ überprüfen. Einer Mißachtung der Erwartungen dieser Kundengruppe droht die Gefahr einer Verdrängung aus diesem Kundensegment, die es wegen der hohen Umsatzchancen bei beschriebener Kundengruppe zu vermeiden gilt.

\section{33 Zusammenfassende Hypothesenbeurteilung}

Eine abschließende Zusammenfassung der auf Kundenebene als erklärungswirksam für die Beurteilungen von Handelsvertretungen abgegrenzten Merkmale deckt - wie bereits auf der Herstellerebene - die Notwendigkeit eines differenzierten Handelsvertreter-Marketing auf. Je nach Schwerpunkt 
der unternehmerischen Tätigkeit von Handelsvertretungen sind die für die einzelnen Dienstleistungsbereiche als beeinflussende Faktoren identifizierten Kundenmerkmale bei der Maßnahmenplanung und Gestaltung zu berücksichtigen. Die Beurteilung der Ausgangshypothesen kann dabei als genereller Leitfaden der einzuschlagenden Vorgehensweise gelten. Abbildung 46 zeigt im Uberblick die hypothesenbezogenen Resultate der Studie zur Einschätzung der Rollenerfüllung sowie der Rollenerwartungen ${ }^{1}$.

Zur Intergruppenhypothese liegen unterschiedliche Resultate vor. Die Beurteilung der gegenwärtigen Leistungserfüllung von Handelsvertretungen wird in den Bereichen Produktanregungen und Preisgestaltung durch unternehmensbezogene Faktoren determiniert; personenbezogene Merkmale dominieren dagegen die Einschätzung des Vertretungsprogramms. Bei der innerbetrieblichen Leistungsfähigkeit weisen beide Variablengruppen gleiche Erklärungsbeiträge auf.

Eine Kenntnis dieser Zusammenhänge versetzt Handelsvertretungen in die Lage, die sich aus einem angepaßten Leistungsangebot ergebenden zukünftigen Beurteilungen bereits bei der Planung dieser Leistungsanpassung $z u$ antizipieren. Die alleinige Beachtung der sich bei den Rollenerwartungen als dominierend erwiesenen unternehmensbezogenen Merkmale ist somit um den impliziten Erklärungswert personaler Merkmale zu relativieren.

In die gleiche Richtung deuten die Ergebnisse der Kombinationshypothese. Die Annahme eines steigenden Erklärungswertes bei der Verknüpfung unternehmensbezogener Merkmale mit personalen Faktoren kann für die Ebene der Kunden in allen Dienstleistungsbereichen als bestätigt gelten.

1 Zur Formulierung der Hypothesen vgl. Kap. B. 3.2 dieser Arbeit. 


\begin{tabular}{|c|c|c|c|c|c|c|}
\hline \multirow[t]{2}{*}{ Hypothese } & \multicolumn{3}{|c|}{ R $\circ 11$ e $n$ e $r$ f u 1 l u n g } & \multicolumn{3}{|c|}{$R \circ 11$ e $n$ e $r w a r t u n g$} \\
\hline & $\begin{array}{l}\text { Intergruppen- } \\
\text { hypothese } \\
\mathrm{H}_{\text {erf1 }}^{\mathrm{K}}\end{array}$ & $\begin{array}{l}\text { Intragruppen- } \\
\text { hypothese } \\
\text { Herf2 }^{\mathbf{K}}\end{array}$ & $\begin{array}{c}\text { Kombinations- } \\
\text { hypothese } \\
\text { Herf3 }^{K}\end{array}$ & $\begin{array}{l}\text { Intergruppen- } \\
\text { hypothese } \\
\mathrm{H}_{\text {erw1 }} \mathrm{K}\end{array}$ & $\begin{array}{c}\text { Intragruppen- } \\
\text { hypothese } \\
\text { Herw2 }^{K}\end{array}$ & $\begin{array}{c}\text { Kombinations- } \\
\text { hypothese } \\
\mathrm{H}_{\text {erw3 }} \mathrm{K}\end{array}$ \\
\hline $\begin{array}{l}\text { Produktanregungen } \\
\text { Preisgestaltung } \\
\text { innerbetriebliche } \\
\text { Leistungsfahigkeit } \\
\text { Vertretungsprogramm }\end{array}$ & $\begin{array}{l}+ \\
+ \\
\pm \\
\pm\end{array}$ & $\begin{array}{l}+ \\
(-) \\
+ \\
+\end{array}$ & $\begin{array}{l}+ \\
+ \\
+ \\
+\end{array}$ & & & \\
\hline $\begin{array}{l}\text { Preisgestaltung } \\
\text { Produktanregungen } \\
\text { innerbetriebliche } \\
\text { Leistungsfähigkeit } \\
\text { Vertretungsprogramm }\end{array}$ & & & & $\begin{array}{l}+ \\
+ \\
+ \\
+\end{array}$ & $\begin{array}{l} \pm \\
- \\
(-)\end{array}$ & $\begin{array}{l}+ \\
+ \\
+ \\
+\end{array}$ \\
\hline
\end{tabular}

Abb. 46: Zusammenfassende Hypothesenbeurteilung zur Rollenerfüllung und Rollenerwartung aus der Sicht der Abnehmer

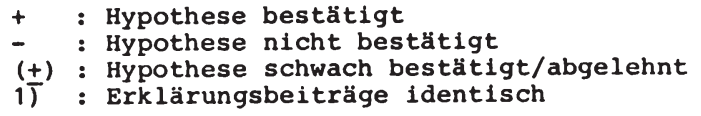


Zwar eignen sich personale Variable allein kaum zur differenzierten Analyse der Kundenbeurteilungen; in der Verknüpfung mit den unternehmensbezogenen Merkmalen ist ihre Aussagekraft dagegen unbestreitbar. Angesichts dieser Befunde gewinnen die personalen Merkmale der Kontaktpartner von Handelsvertretungen bei den Abnehmern eine besondere Bedeutung für die bei der Intergruppenhypothese beschriebene Antizipation zukünftiger Beurteilungen.

Zur Beantwortung der Frage, welche unternehmensbezogenen Faktoren einen herausragenden Einfluß für die Einschätzungen von Handelsvertretungen aufweisen, stellt die Intragruppenhypothese ambivalente Ergebnisse bereit. Für die Beurteilung des gegenwärtigen Leistungsangebotes von Handelsvertretungen dominieren - mit einer leichten Abschwächung bei der Preisgestaltung - in allen Dienstleistungsbereichen einkaufsorientierte Faktoren die Kundenbeurteilungen. Die Erwartungen der Kunden an die Rolle von Handelsvertretungen werden dagegen nicht durch Merkmale ihres Einkaufs sondern durch allgemeine unternehmensdemographische Faktoren geprägt.

Für die strategische Ausrichtung eines HandelsvertreterMarketing kann demnach auf eine Berücksichtigung einkaufsorientierter Merkmale nicht verzichtet werden. Diese Aussage erklärt sich aus den Resultaten der Studie, die in einigen Dienstleistungsbereichen das allgemeine Merkmal 'Betriebsform' als erklärungswirksam identifiziert haben.

Mit der Betriebsform eines Abnehmers ist in vielen Fällen zugleich eine Verbindung zu einkaufsbezogenen Merkmalen aufgezeigt. Die unterschiedliche Art der Einkaufsentscheidung oder die Bezugsquellenstruktur im Vergleich von Facheinzelhandel und Einkaufsverband verdeutlichen beispielhaft die- 
sen Zusammenhang. Für das Handelsvertreter-Marketing leitet sich aus diesen Resultaten die Erfordernis ab, die für alternative Kundengruppen als Bestimmungsfaktoren herausgearbeiteten allgemeinen und einkaufsbezogenen Merkmale der Abnehmer bei der Maßnahmenplanung $z u$ berücksichtigen.

4. Analyse der Rollenbeziehungen im HandelsvertreterMarketing

Die Resultate zur Rollenerfüllung und Rollenerwartung auf Kundenebene vervollständigten die Diskussion über einzelne Komponenten einer Rollenanalyse im Handelsvertreter-Marketing. Die bisherige Form der Analyse operationalisierter Rollenkonstrukte auf den drei Betrachtungsebenen Handelsvertretung, Hersteller und Abnehmer stellt damit das Grundgerüst weiterführender Interpretationen zur Rolle von Handelsvertretungen im vertikalen Marketing dar.

Die separate Diskussion einzelner Rollenkomponenten hat vor allem auf der Hersteller- und Kundenseite mehrfach Zusammenhänge und wechselseitige Abhängigkeiten zwischen der Beurteilung der Rollenerfüllung und den Rollenerwartungen erkennbar werden lassen. Eine integrative Betrachtung der Rollenkomponenten dieser beiden Modellebenen ist daher angezeigt.

Nicht zuletzt die Chance zur Ableitung differenzierterer Aussagen für das Handelsvertreter-Marketing unterstützt die Vorgehensweise einer unmittelbaren Gegenüberstellung von Rollenerfüllung und Rollenerwartung. Diese als horizontale Rollenbeziehungen vorgestellten Zusammenhänge 
und die daraus abgeleiteten Hypothesen ${ }^{1}$ sowie die Beziehungen zwischen den Marktpartnern (vertikale Rollenbeziehungen) werden nachfolgend hinsichtlich ihrer empirischen Erscheinungs formen eingehender beleuchtet.

\section{1 Horizontale Rollenbeziehungen}

Gemäß den Grundüberlegungen zur Rollenanalyse im Handelsvertreter-Marketing treten horizontale Rollenbeziehungen in der Ebene der vertretenen Unternehmen und der Abnehmer von Handelsvertretungen auf (vgl. Abbildung 9). Art bzw. Ausma $B$ dieser Beziehungen sind dabei durch den Unterschied zwischen der Beurteilung der Rollenerfüllung und den Rollenerwartungen der Marktpartner gekennzeichnet. Diese Diskrepanz - bezeichnet als Grad der Rollenerfüllung - gibt Auskunft über die Frage, in welchem Maße eine Handelsvertretung die an ihre Position geknüpfte Rolle zu erfüllen vermag. Für die Planung von Maßnahmen im HandelsvertreterMarketing sind daher die Resultate zum Grad der Rollenerfüllung unmittelbar verwendbar. Im folgenden werden Ausprägungen und Einflußgrößen des Grades der Rollenerfüllung als Ausdruck horizontaler Rollenbeziehungen für Herstellerund Kundenebene aufgezeigt.

\subsection{Rollenbeziehungen auf Herstellerebene}

Die Erfassung horizontaler Rollenbeziehungen erfolgte durch eine Gegenüberstellung der Beurteilungen der Rollenerfüllung und der Rollenerwartungen der Hersteller in allen Dienstleistungsbereichen von Handelsvertretungen. Zur Strukturierung der vielfältigen Dienstleistungen wurde da-

$1 \mathrm{Vgl}$. Kapitel B. 1.3 und B. 3.3 dieser Arbeit. 
bei auf die empirisch verdichtete Dienstleistungsstruktur zurückgegriffen ${ }^{1}$.

Die konkret-rechnerischen Ermittlung des Grades der Rollenerfüllung faßt die Distanzen zwischen den jeweiligen Dienstleistungsbereichen bei Rollenerfüllung und Rollenerwartung $\mathrm{zu}$ einem Indexwert zusammen ${ }^{2}$. Diese Maßgröße ist geeignet, unterschiedliche Grade der Rollenerfüllung auszudrücken.

Für die empirisch ermittelten Indexwerte wurde zur Veranschaulichung eine Unterteilung in drei Gruppen vorgenommen. Demnach soll von einem hohen Grad der Rollenerfüllung gesprochen werden, wenn die Beurteilungen von Rollenerfüllung und Rollenerwartungen übereinstimmen, d.h. keine Distanzen zwischen diesen Rollenkomponenten auftreten. Zwei weitere Abstufungen kennzeichnen einen mittleren bzw. einen niedrigen Grad der Rollenerfüllung ${ }^{3}$. Mit Hilfe dieser Kategorien lassen sich Unterschiede in einzelnen Dienstleistungsbereichen von Handelsvertretungen wirksam voneinander abgrenzen.

1 Vgl. Kapitel C. 2. dieser Arbeit.

2 Diese Vorgehensweise ähnelt in ihrer Grundstruktur den in der Käuferverhaltensforschung diskutierten Idealpunktmodellen. Zur Anwendung und Aussagefähigkeit vgl. insbes. Trommsdorff, V., Die Messung von Produktimages für das Marketing. Grundlagen und Operationalisierung, Köln usw. 1975, S. 67 ff.; Andritzky, K., Die Operationalisierbarkeit von Theorien zum Konsumentenverhalten, Berlin 1976, S.74 ff.. Zur Indexbildung vgl. Wettschureck, G., MeBtechnisches Praktikum für Marktforscher, Hamburg 1977,S.57 ff. sowie Berekoven, L.,Eckert,W., Ellenrieder,P.,a.a.O., S. 134 f..

3 Zur Ermittlung dieser Kategorien wurden Mittelwert und Standardabweichung des Index herangezogen:

-hoher Grad der Rollenerfüllung: Inde $\mathrm{x}=0$

- mittlerer Grad der Rollenerfüllung: $0>$ Index $\leqslant$ (Indexmittelwert + -standardabweichung)

- niedriger Grad der Rollenerfüllung: Index $>$ (Indexmittelwert + -standardabweichung) 


\subsection{Ausprägungen der Rollenbeziehungen}

Die für die beschriebenen Indexkategorien ermittelte Aufteilung der vertretenen Unternehmen zeigt Abbildung 47 für alle Dienstleistungsbereiche von Handelsvertretungen.

\begin{tabular}{|l|c|c|c|}
\hline \multicolumn{1}{|c|}{$\begin{array}{c}\text { Grad der } \\
\text { Rollenerfüllung }\end{array}$} & hoch & mittel & niedrig \\
\hline Dienstleistungsbereich & 6,0 & 80,2 & 13,8 \\
Kundenbetreuung & 3,0 & 87,9 & 9,1 \\
$\begin{array}{l}\text { Produktanregungen } \\
\text { Preisgestaltung und Leistungs- } \\
\text { vergütung }\end{array}$ & 6,5 & 83,9 & 9,6 \\
$\begin{array}{l}\text { Ausstattung der Handelsvertre- } \\
\text { tung }\end{array}$ & 18,9 & 74,9 & 6,2 \\
\begin{tabular}{l} 
Information an Hersteller \\
\hline
\end{tabular}
\end{tabular}

Abb. 47: Ausprägungen des Grades der Rollenerfüllung von Handelsvertretungen aus Herstellersicht (in \&)

Ein globaler Blick auf die empirischen Befunde erweckt den Eindruck, Handelsvertretungen sind - aus Herstellersicht in vorwiegend mittlerem Ausma $B$ dazu fähig, ihre Rolle im Absatzkanal auszufüllen. Je nach Dienstleistungsbereich sind zwischen 69 \& und knapp 88 \& der vertretenen Unternehmen dieser Mittelkategorie zuzuordnen. Diese wenig 
trennscharfe und damit wenig befriedigende Aussage für das Handelsvertreter-Marketing kann durch beide Kategorien hoher bzw. niedriger Ausprägungen des Grades der Rollenerfüllung entscheidend verbessert werden.

Die Resultate $z u$ hohen Graden der Rollenerfüllung in den verschiedenen Dienstleistungsbereichen decken eine dreigeteilte Zufriedenheit der Hersteller mit dem Leistungsangebot von Handelsvertretungen auf. Dabei erfahren die Handelsvertretungen in den Leistungsbereichen Preisgestaltung und Leistungsvergütung sowie bei der Ausstattung der Handelsvertretung die weitaus besten Beurteilungen. Immerhin 19 \& aller befragten Unternehmen sehen in diesen beiden Leistungsbereichen keinen Unterschied zwischen der Rollenerfüllung und der Rollenerwartung. Die vergleichsweise beste Einschätzung erfahren die Handelsvertretungen bei der Ausstattung ihres eigenen Geschäftes; lediglich 6,2 \& der vertretenen Unternehmen konstatieren einen niedrigen Grad der Rollenerfüllung in diesem Bereich. Dieser Anteil ist für die Aktivitäten der Preisgestaltung und Leistungsvergütung nahezu doppelt so hoch $(11,8 \%)$. Handelsvertreter müssen daher im Vergleich der beiden genannten Dienstleistungsbereiche den Aktivitäten der Preisgestaltung und Leistungsvergütung priorität einräumen.

In deutlichem Abstand $z u$ den beiden vorgestellten Dienstleistungsbereichen bilden Informationsverarbeitung und Produktanregungen eine mittlere Gruppe hinsichtlich der Zufriedenheit in der Einschätzung durch die Hersteller. 6 \& der vertretenen Unternehmen weisen eine positive Einschätzung der Handelsvertretungen auf. Bei der Gestaltung des Dienstleistungsangebotes ist dabei der Verarbeitung von Hersteller- und Kundeninformationen besondere Aufmerksamkeit zu widmen, weil nahezu 14 \& der ver- 
tretenen Unternehmen in diesem Bereich ein geringes Maß an Rollenerfüllung bei den Handelsvertretungen feststellen.

Eine dritte Gruppe von Dienstleistungen hinsichtlich einer ähnlichen Ausprägung des Grades der Rollenerfüllung bilden die Kundenbetreuung und die herstellergerichteten Informationen. Beide Leistungsbereiche stellen mit nur 3 \& zufriedener Hersteller einen Schwachpunkt der Handelsvertreteraktivitäten aus der Sicht der vertretenen Unternehmen dar. In besonderem Maße gilt dies für die Informationen an die vertretenen Unternehmen; hier liegt mit über 18 \& zugleich die größte Gruppe von Herstellern mit niedriger Einschätzung der Rollenerfüllung von Handelsvertretungen ${ }^{1}$.

Abbildung 48 veranschaulicht die diskutierten Zusammenhänge für die einzelnen Dienstleistungsbereiche von Handelsvertretungen. Die Graphik visualisiert in ihrem unteren Bereich die Leistungsmerkmale von Handelsvertretungen hinsichtlich eines hohen Grades der Rollenerfüllung. Eine Ausweitung dieses Herstellerkreises, der tendenziell eine hohe zufriedenheit mit der Rolle von Handelsvertretungen aufweist, kann als generelle zielrichtung eines Handelsvertreter-Marketing gelten.

Betrachtet man die Graphik von ihrem oberen Ende, werden die Bereiche mit niedrigem Grad der Rollenerfüllung im Vergleich veranschaulicht. Eine Reduzierung des Anteils der vertretenen Unternehmen mit derart niedrigen Einschätzungen der Rolle von Handelsvertretungen bildet eine korrespondierende Zielgröße zur Verbesserung der Position von

1 Dieses differenzierte Resultat bestätigt damit die bereits bei globaler Analyse erkennbare Tendenz; vgl. Meffert, H., Die Information ist verbesserungsfähig, a.a.O., S. 1439 f. sowie Kimmeskamp, G., Die Handelsvertretung im Meinungsbild von Industrie und Handel, a.a.O., S. 4. 


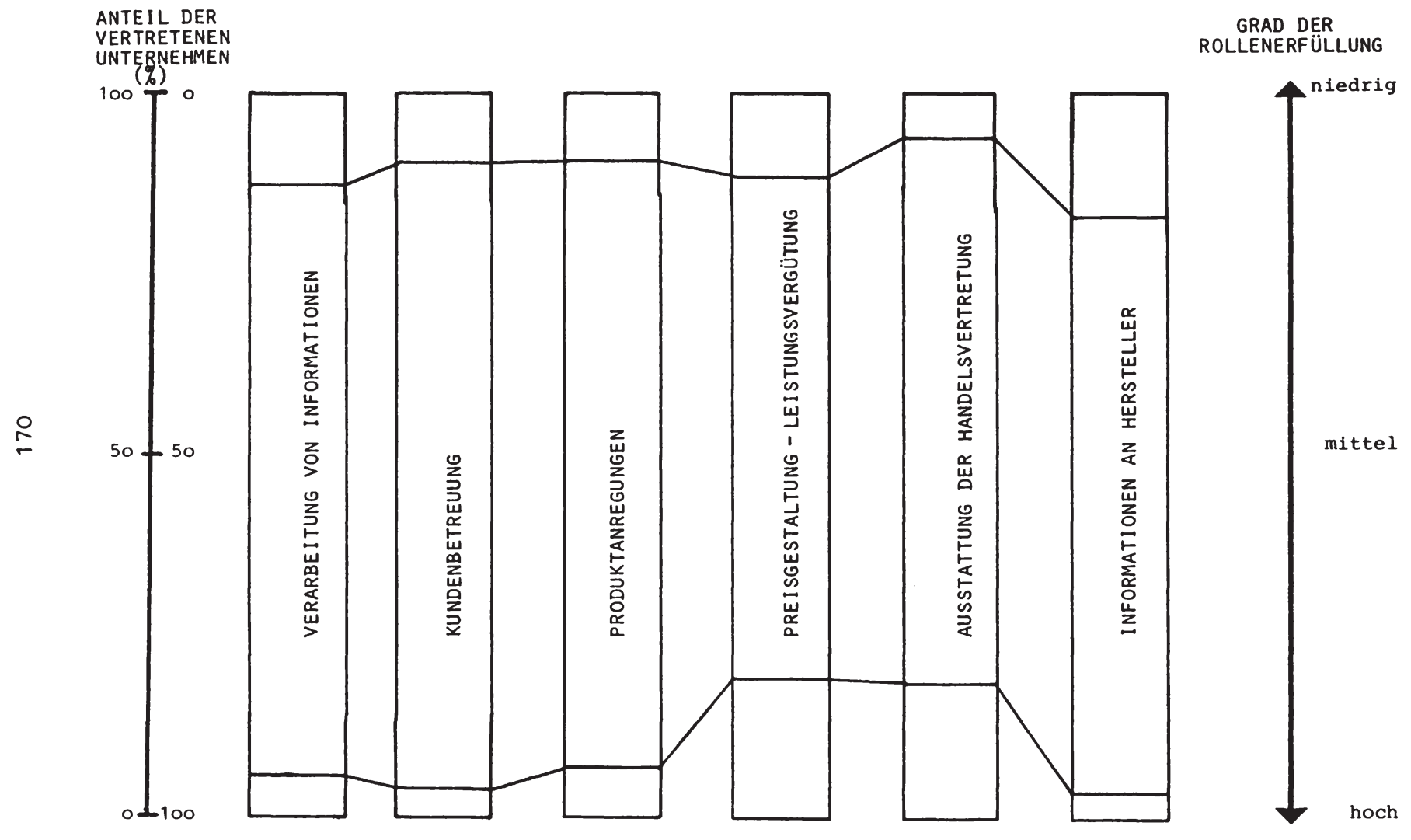

Abb. 48: Grad der Rollenerfüllung in einzelnen Dienstleistungsbereichen aus Herstellersicht 
Handelsvertretungen. Ausmaß der Zielerreichung, zeitliche Durchführbarkeit und insbesondere prioritäten einzelner Leistungsbereiche ${ }^{1}$, die sich aus der dualen Einbindung von Handelsvertretungen in den Absatzkanal ergeben, erfordern zu ihrer Festlegung eine Kenntnis der Faktoren, die auf der Ebene der vertretenen Unternehmen die Beurteilungen der Hersteller maßgeblich beeinflußt haben. Aus diesem Grunde werden nachfolgend die Bestimmungsfaktoren des Grades der Rollenerfüllung auf Herstellerebene empirisch herausgearbeitet.

\subsection{Bestimmungsfaktoren der Rollenbeziehungen}

Die Gewinnung der Merkmale der vertretenen Unternehmen, die die Einschätzung des Grades der Rollenerfüllung beeinflussen, erfolgte analog zur Analyse der einzelnen Rollenkomponenten mittels multipler Regressionen ${ }^{2}$. Zur Ubberprüfung der theoriegeleiteten Hypothesen über den Einfluß der unterschiedlichen Determinantengruppen wurden erneut die entsprechenden variablengruppenbezogenen Regressionen durchgeführt. Abbildung 49 stellt die Resultate für die einzelnen Dienstleistungsbereiche im Uberblick dar.

Im Vergleich zur Analyse der einzelnen Rollenkomponenten auf Herstellerebene fällt unmittelbar auf, daß der Grad der Rollenerfüllung - und damit die integrative Betrachtung von gegenwärtiger Leistungserfüllung und Rollenerwartungen - durch eine erheblich größere Anzahl der unabhängigen Merkmale erklärt werden kann. Dieses zunächst im

$1 \mathrm{Zu}$ diesen Zieldimensionen vgl. Meffert, H., Marketing, a.a.O., S. $72 \mathrm{ff.;}$ Probleme der Zielbildung im vertikalen Marketing belegen Rosenbloom, B., a.a.0., S. 96 f. sowie Steffenhagen, H., Konflikt und Kooperation in Absatzkanälen, a.a.O., S. 45 f.; Maas, R.M., a.a.O., S. 116 ff..

2 Zum methodischen Vorgehen vgl. Kapitel C. 3.11 dieser Arbeit. 


\begin{tabular}{|c|c|c|c|c|c|}
\hline \multirow{2}{*}{$\begin{array}{l}\text { Dienstleistungs- } \\
\text { bereich }\end{array}$} & $\begin{array}{l}\text { allgemeine } \\
\text { Unternehmens- } \\
\text { demographie } \\
\text { (1) }\end{array}$ & $\begin{array}{l}\text { vertriebsbe- } \\
\text { zogene } \\
\text { Merkmale } \\
\text { (2) }\end{array}$ & $\begin{array}{l}\text { unternehmens- } \\
\text { bezogene Merk- } \\
\text { male gesamt } \\
\begin{array}{l}\text { (1) }+ \text { (2) } \\
\text { (3) }\end{array}\end{array}$ & $\begin{array}{l}\text { personenbe- } \\
\text { zogene } \\
\text { Merkmale } \\
\quad(4) \\
\end{array}$ & $\begin{array}{l}\text { unternehmens- } \\
\text { und personen- } \\
\text { bezogene } \\
\text { Merkmale } \\
\begin{array}{c}(3)+(4) \\
(5)\end{array}\end{array}$ \\
\hline & \begin{tabular}{l|l}
$n_{v}$ & $r$ \\
\end{tabular} & \begin{tabular}{l|l}
$n_{v}$ & $r$
\end{tabular} & $n_{v} \quad r$ & \begin{tabular}{l|l}
$n_{v}$ & $r$ \\
\end{tabular} & \begin{tabular}{l|l}
$n_{\mathrm{v}}$ & $\mathrm{r}$ \\
\end{tabular} \\
\hline In formationsverarbeitung & $120,31^{++}$ & $4 \mid 0,18^{+}$ & $16 \mid 0,36^{t+}$ & $7 \mid 0,25^{++}$ & $0,43^{++}$ \\
\hline Kundenbetreuung & $0,31^{++}$ & $810,30^{++}$ & $\left.15\right|^{0,42^{++}}$ & $2 \mid 0,18^{++}$ & $\left.14\right|_{0,43^{++}}$ \\
\hline Produktanregungen & $7: 0,24^{++}$ & $410,23^{++}$ & $101^{0,31^{++}}$ & $610,24^{++}$ & $18 \mathrm{I}_{0,41^{++}}$ \\
\hline $\begin{array}{l}\text { Preisgestaltung und } \\
\text { Leistungsvergütung }\end{array}$ & $510,24^{++}$ & $\left.\right|_{1} ^{0,23^{++}}$ & $1110,32^{++}$ & $\left.2\right|_{0,14^{+}}$ & $\left.16\right|_{0,37^{++}} ^{\mid}$ \\
\hline $\begin{array}{l}\text { Ausstattung der Handels- } \\
\text { vertretung }\end{array}$ & $91_{10,36^{++}}$ & $41_{0,19^{++}}$ & $\left.11\right|_{0,39^{++}}$ & $\left.1\right|_{0,11^{+}}$ & $13 \mid 0,41^{++}$ \\
\hline Information an Hersteller & $13 \mid 0,38^{++}$ & $6: 0,25^{++}$ & $210,44^{++}$ & $\left.3\right|^{0,16^{+}}$ & $24 \mid 0,46^{++}$ \\
\hline
\end{tabular}

Abb. 49: Bestimmungsfaktoren horizontaler Rollenbeziehungen auf Herstellerebene

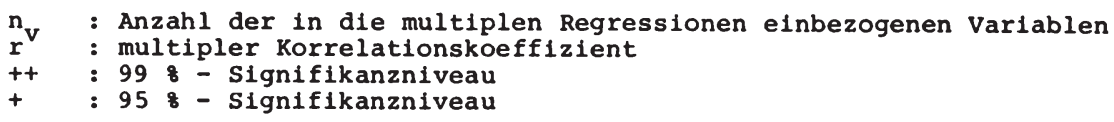


Hinblick auf abzuleitende Implikationen positiv anmutende Ergebnis muß jedoch relativiert werden, weil sich die größere Zahl erklärungswirksamer Variablen nicht in einer Erhöhung der Erklärungsbeiträge niederschlägt. Die multiplen Korrelationskoeffizienten bewegen sich vielmehr in den bekannten Größenordnungen der Analyse einzelner Rollenkomponenten.

Für die vorliegende Problemstellung birgt dieses Resultat eine zweifache Konsequenz. Auf der einen Seite gilt, aus der Vielzahl der als erklärungswirksam identifizierten Variablen diejenigen herauszufiltern, die für das Handelsvertreter-Marketing unmittelbar relevant sind. Die dabei notwendige Selektion von Variablen wirft auf der anderen Seite die Frage nach einem geeigneteren Weg zur empirischen Fundierung eines Handelsvertreter-Marketing auf, der bestehende Interdependenzen weniger stark zerschneidet.

Betrachtet man die Dienstleistungsbereiche im einzelnen, belegen die Ergebnisse zur Informationsverarbeitung einen interessanten Aspekt für die Handelsvertretungen, deren vertretene Unternehmen einen gemischten Vertrieb aufgebaut haben. Hersteller, die Handelsvertreter und Reisende einsetzen, beurteilen den Grad der Rollenerfüllung um so höher, je größer die Anzahl der Reisenden ist. Handelsvertreter kommen demnach den Erwartungen der vertretenen Unternehmen an die Informationsverarbeitung bei herstellerdominierten Vertriebswegen näher als bei vorwiegend vertraglichem Vertrieb. Entweder eine Profilierung der Handelsvertreter oder möglicherweise schlechte Erfahrungen mit Reisenden haben die Handelsvertretungen in diesem 
Leistungsbereich in eine Position versetzt, die eine gute Ausgangsbasis zur langfristigen Absicherung auf der Grundlage fachlicher Kompetenz bereitstellt.

Die für die Kundenbetreuung beschriebene Schwachstelle d.h. mangelnde Ausfüllung der Rolle - aus Herstellersicht läßt sich durch die Einbeziehung ihrer Bestimmungsfaktoren präzisieren. Die empirischen Befunde belegen eine tendenziell hohe Diskrepanz zwischen aktuellem Leistungsangebot und Herstellererwartungen bei solchen vertretenen Unternehmen, die viele Handelsvertreter einsetzen und die insgesamt lange mit Handelsvertretungen zusammenarbeiten. Auch Hersteller mit einem hohen Umsatzanteil aus Direktgeschäften weisen dieses Resultat auf. Hersteller mit den beschriebenen Merkmalen neigen dabei tendenziell dazu, die Handelsvertreter durch Reisende abzulösen. Die Gefahr für Handelsvertretungen schwächt sich jedoch ab, je mehr Reisende ein Hersteller einsetzt; in diesem Fall entsprechen die Leistungen der Handelsvertreter in höherem Maße den Erwartungen der Hersteller. Einer drohenden Vertragskündigung muß somit vorrangig durch entsprechende Anstrengungen im Bereich der Kundenbetreuung entgegengewirkt werden.

Die bei der Informationsverarbeitung und der Kundenbetreuung erkennbare Nische zur Profilierung für Handelsvertreter bei vertretenen Unternehmen mit gemischtem Vertrieb findet sich in ähnlicher Form im Leistungsbereich der produktanregungen sowie bei den herstellergerichteten Informationen. Auch in diesen Dienstleistungsbereichen können Handelsvertreter die als tendenziell positiv anzusehende Ausfüllung ihrer Rolle durch entsprechende Maßnahmen weiter festigen bzw. ausbauen, um im Wettbewerb mit den Reisenden zu bestehen. 
Bei der Vorstellung der Ausprägungen des Grades der Rollenerfüllung haben die Ausstattung der Handelsvertretungen in materieller und personeller Hinsicht sowie die Aktivitäten der Preisgestaltung und Leistungsvergütung die vergleichsweise beste Beurteilung durch die Hersteller erfahren. Neben allgemeinen Merkmalen zur Rechtsform sowie regionalen Ansiedlung der Hersteller lassen sich auch diese Beurteilungen durch vertriebsbezogene Einflußgrößen weiter differenzieren. Demnach wächst der Grad der Rollenerfüllung von Handelsvertretungen hinsichtlich der Aktivitäten der Preisgestaltung und Leistungsvergütung bei Herstellern der Investitionsgüterbranche, die in hohem Maße Reisende einsetzen. Steigen dagegen die Umsatzanteile der Handelsvertreter oder aus Direktgeschäften, können die Handelsvertreter den Erwartungen der vertretenen weitaus weniger gerecht werden. Lediglich Handelsvertretungen, die bereits lange mit den vertretenen Unternehmen zusammenarbeiten, konnten eine positivere Einschätzung der Hersteller auf sich ziehen. Hinsichtlich der Ausstattung von Handelsvertretungen gilt diese Argumentation in ihrer Grundrichtung vor allem für Handelsvertreter der Konsumgüterbranche und bei industriellen Ge- und Verbrauchsgütern.

Betrachtet man die Analyse der Bestimmungsfaktoren zusammenfassend, so konnte die empirische Untersuchung eine Reihe von Faktoren herausarbeiten, die einen Einfluß darauf haben, in welchem AusmaB eine Handelsvertretung den Erwartungen der Hersteller an ihre Rolle im Absatzkanal gerecht wird. Die Ergebnisse belegten dabei wiederholt die Notwendigkeit einer differenzierten Maßnahmengestaltung gegenüber den vertretenen Unternehmen (z.B. hinsichtlich der Unternehmensgröße oder Vertriebsstruktur). Damit wird das Prinzip eines differenzierten - d.h. auf die Belange 
unterschiedlicher Zielgruppen abgestimmten - Einsatzes der Marketinginstrumente für das Handelsvertreter-Marketing zu einem empirischen Erfordernis ${ }^{1}$. In den Gedanken eines solchermaßen zielgruppenorientierten HandelsvertreterMarketing sind die Einschätzungen der Kunden zum Grad der Rollenerfüllung als zweiter wesentlicher Bestandteil aufzunehmen.

\subsection{Rollenbeziehungen auf Kundenebene}

\subsection{Ausprägungen der Rollenbeziehungen}

In gleicher Weise wie auf der Ebene der vertretenen Unternehmen läßt sich auf der Grundlage der empirischen Studie der Grad der Rollenerfüllung von Handelsvertretungen aus der Sicht ihrer Abnehmer ermitteln. Auch hier diente die zuvor ermittelte Dienstleistungsstruktur auf empirischer Basis als Ausgangspunkt der Indikatorermittlung ${ }^{2}$. Abbildung 50 belegt, wie sich die Einschätzungen der Kunden hinsichtlich des Grades der Rollenerfüllung von Handelsvertretungen verteilen.

1 Zum Prinzip des differenzierten Marketing vgl. Meffert, H., Marketing, a.a.O., S. 225 f., Kotler, P., a.a.0., s. $182 \mathrm{f}$..

2 Zur Dienstleistungsstruktur vgl. Kapitel C. 2.22; zur Indexbildung Kapitel C. 4.11. 


\begin{tabular}{|l|c|c|c|}
\hline \multicolumn{1}{|c|}{$\begin{array}{c}\text { Grad der Rollener- } \\
\text { füllung }\end{array}$} & mittel & niedrig \\
$\begin{array}{l}\text { Dienstleistungs- } \\
\text { bereich }\end{array}$ & hoch & 76,7 & 9,8 \\
\hline $\begin{array}{l}\text { Preisgestaltung } \\
\text { Produktanregungen }\end{array}$ & 13,5 & 79,8 & 16,1 \\
$\begin{array}{l}\text { innerbetriebliche Leistungs- } \\
\text { fähigkeit }\end{array}$ & 7,3 & 79,7 & 13,0 \\
Vertretungsprogramm & 6,5 & 83,0 & 10,5 \\
\hline
\end{tabular}

Abb. 50: Ausprägungen des Grades der Rollenerfüllung aus Kundensicht (in o )

Ahnlich den Beurteilungen der Hersteller werden die Handelsvertretungen ihrer Rolle auch aus Kundensicht in vorwiegend mittlerem Maße gerecht. Dennoch gestatten die Kategorien hoher bzw. niedriger Ausprägungen des Grades der Rollenerfüllung eine Differenzierung innerhalb des gesamten Leistungsangebotes. Abbildung 51 veranschaulicht die zu diskutierenden Zusammenhänge in graphischer Form.

Im Rahmen der preisgestaltung erfahren die Handelsvertretungen eine insgesamt positive Beurteilung. 13,5 \& der befragten Abnehmer sehen hier keine Diskrepanzen zwischen dem aktuellen Leistungsangebot (beispielsweise den Handlungsspielräumen für die Preisfestlegung) und ihren Erwartungen; lediglich knapp $10 \%$ der Abnehmer halten diesen Leistungsbereich für weniger adäquat ausgefüllt. Handels- 


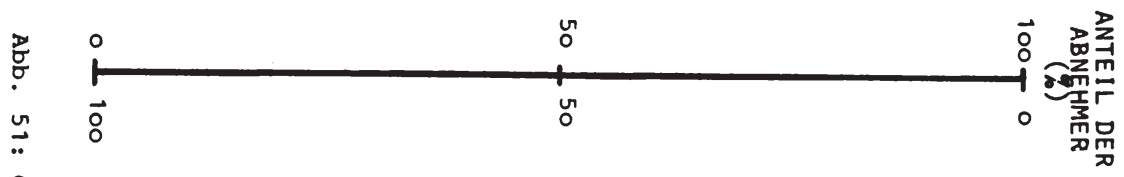

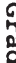

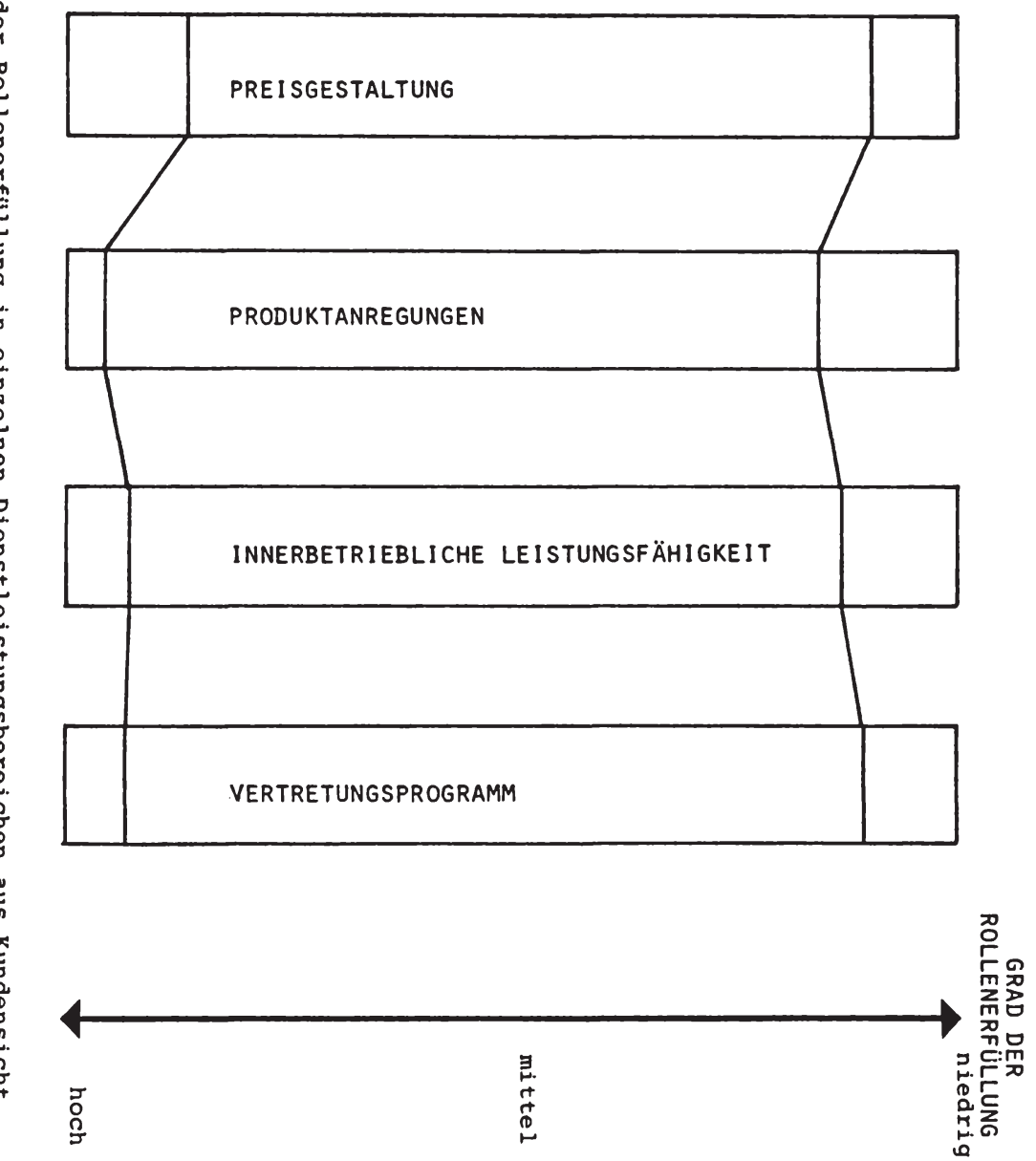


vertretungen sind daher gehalten, die erkennbare Konsolidierung im Leistungsbereich der Preisgestaltung weiter auszubauen. Gegenüber den vertretenen Unternehmen läßt sich dabei das Argument unterstützend heranziehen, daß eine Modifizierung der Preis- und Konditionenverantwortung den Erwartungen der Abnehmer noch besser $\mathrm{zu}$ entsprechen vermag und damit die Position von Hersteller und Handelsvertretung am Markt festigt.

Weitaus schlechter wird dagegen die Rolle der Handelsvertretungen bei den produktanregungen eingeschätzt. Nur 4 \& der Abnehmer sehen ihre Interessen durch die derzeitige Leistungserfüllung der Handelsvertretungen zur Einflußnahme auf das Angebotsprogramm der Hersteller wirksam vertreten. 16 \& der Kunden halten diesen Leistungsbereich für besonders schlecht repräsentiert. Diese Resultate unterstreichen damit die Bedeutung, die der Beeinflussung des Herstellerangebotes durch die Handelsvertretungen aus Kundensicht zukommt, wie dies bereits bei der isolierten Analyse der Rollenkomponenten auf Abnehmerebene transparent wurde. Die empirische Studie läßt darauf schließen, daß es die Handelsvertretungen noch nicht verstanden haben, ihre Position als Mittler zwischen Industrie und Handel im Hinblick auf ein kundenorientiertes Produktangebot auszufüllen.

Eine leichte Abschwächung dieses Mangels verzeichnen die Resultate zum Vertretungsprogramm. Hier geben die tendenziell günstigeren Einschätzungen der Kunden Anlaß zu der Vermutung, daß die Handelsvertretungen zumindest versuchen, mangelnde Kundenorientierung im Produkt- und Sortimentsangebot der Hersteller durch eine entsprechende Zusammensetzung ihres Vertretungsprogramms auszugleichen. 
Verstärkte Anstrengungen in diesem Leistungsbereich in Verbindung mit höherem Aktivitätsniveau bei den Produktund Sortimentsanregungen gegenüber den vertretenen Unternehmen bieten nach der vorliegenden studie alle Voraussetzungen einer Profilierung auf Kundenebene. Leistungsverbesserungen der beschriebenen Art bergen dabei wenig Konfliktpotential in sich, weil auch die vorgestellten Beurteilungen der Hersteller in dieselbe Richtung deuten.

Der Grad der Rollenerfüllung wird weiterhin bei der innerbetrieblichen Leistungsfähigkeit von ca. $7 \%$ der befragten Abnehmer für ausreichend angesehen, während $13 \%$ der Kunden die Rolle von Handelsvertretungen in diesem Bereich nur unzureichend ausgefüllt sehen. Im Vergleich zu den Herstellerbeurteilungen schätzen die Kunden beispielsweise die Erreichbarkeit der Handelsvertreter sowie die Qualifikation ihrer Mitarbeiter erheblich schlechter ein. Möglicherweise führen häufigere Kontakte zwischen Handelsvertretung und Kunde - im Vergleich zu den Herstellerbeziehungen - zu dieser Beurteilung, wobei außerdem tendenziell konfliktträchtigere Aspekte $\mathrm{zu}$ behandeln sind (beispielsweise verspätete Lieferungen oder Reklamationen).

An diesem Beispiel wird erneut die zweiseitige Ausrichtung des Handelsvertreter-Marketing offenkundig. Ein befriedigendes Leistungsniveau aus Herstellersicht deckt sich dabei nicht notwendigerweise mit den Erwartungen der Kunden an die Rolle von Handelsvertretungen. Eine sorgfältige $\mathrm{Ab}-$ wägung von Maßnahmen der Leistungsgestaltung oder -veränderung im Hinblick auf mögliche Reaktionen beider Marktpartner ist unumgänglich. Wertvolle Hilfestellung liefert dazu die Kenntnis der Faktoren, die auf der Kundenebene einen EinfluB auf die Beurteilungen der Abnehmer ausüben. 


\subsection{Bestimmungsfaktoren der Rollenbeziehungen}

Die für die horizontalen Rollenbeziehungen - d.h. den Grad der Rollenerfüllung - auf Kundenebene als erklärungswirksam abgegrenzten Merkmale stellt Abbildung 52 im Uberblick vor $^{1}$. Ahnlich zur Herstellerebene erweist sich auch bei den Abnehmern eine tendenziell größere Anzahl von Variablen als erklärungswirksam, wobei sich jedoch die Erklärungsbeiträge der einzelnen Variablengruppen in ähnlicher Höhe wie bei der Analyse einzelner Rollenkomponenten bewegen. Eine Verbesserung der Aussagefähigkeit der empirischen Resultate kann somit für die integrative Betrachtung von Rollenerfüllung und Rollenerwartung nicht festgestellt werden.

Hinsichtlich des Erklärungsgehaltes der verschiedenen Merkmalsgruppen erfahren bisherige Ergebnisse weitgehend eine Bestätigung. Demnach wirken allgemeine unternehmensdemographische Merkmale in höherem Maße auf den Grad der Rollenerfüllung. ein als einkaufsbezogene Faktoren der Kunden (Spalten 1 und 2). Eine Erklärungswirksamkeit organisatorischer Merkmale des Einkaufs (z.B. Art der Einkaufsentscheidung, Bezugsquellenstruktur) stellt sich erst bei gleichzeitiger Betrachtung im Verbund mit den allgemeinen Unternehmensmerkmalen ein (Spalte 3). Wie bisher sind personenbezogene Merkmale allein kaum aussagefähig (Spalte 4); erst die Verbindung mit den unternehmensorientierten Merkmalen vermag den Erklärungsgehalt personaler Faktoren aufzudecken (Spalte 5).

1 Die Ermittlung erfolgte - wie auf Herstellerebene durch multiple Regressionen. 


\begin{tabular}{|c|c|c|c|c|c|}
\hline Dienstleistungs- & $\begin{array}{l}\text { allgemeine } \\
\text { Unternehmens- } \\
\text { demographie } \\
(1)\end{array}$ & \begin{tabular}{|l} 
einkaufsbe- \\
zogene \\
Merkmale \\
$(2)$
\end{tabular} & $\begin{array}{l}\text { unternehmens- } \\
\text { bezogene } \\
\text { Merkmale ge- } \\
\text { samt } \\
(1)+\text { (2) } \\
(3)\end{array}$ & $\begin{array}{l}\text { personenbe- } \\
\text { zogene } \\
\text { Merkmale } \\
(4)\end{array}$ & $\begin{array}{l}\text { unternehmens- } \\
\text { und personen- } \\
\text { bezogene Merk- } \\
\text { male } \\
(3)+\text { (4) } \\
(5)\end{array}$ \\
\hline bereich & $n_{v} \mid r$ & $n_{v} r$ & $n_{v} \quad r$ & \begin{tabular}{l|l}
$n_{v}$ & $r$
\end{tabular} & $n_{v} \mid r$ \\
\hline Preisgestaltung & $\left.9\right|^{0,29^{+}}$ & $\left.5\right|_{0,25^{+}}$ & $11 \mid 0,35^{++}$ & $-1-$ & $13 \mid 0,38^{t+}$ \\
\hline Produktanregungen & $\left.10\right|^{10,36^{++}}$ & $51^{0,30^{+t}}$ & $\left.17\right|_{0,49^{++}}$ & $\left.3\right|^{1} 0,21^{+}$ & $0,51^{++}$ \\
\hline $\begin{array}{l}\text { innerbetriebliche } \\
\text { Leistungsfähigkeit }\end{array}$ & $\left.7\right|^{0,26^{+}}$ & $61_{0,26^{++}}^{1}$ & $13 \mid 0,38^{++}$ & $4 \mathrm{l}_{0,23^{+}}$ & $\left.17\right|^{1} 0,51^{++}$ \\
\hline Vertretungsprogramm & $11 \mid 0,37^{++}$ & $5 \quad 10,25^{++}$ & $\left.12\right|_{0,41^{++}}$ & $-1-$ & $\left.14\right|^{0,45^{++}}$ \\
\hline
\end{tabular}

Abb. 52: Bestimmungsfaktoren horizontaler Rollenbeziehungen auf Kundenebene

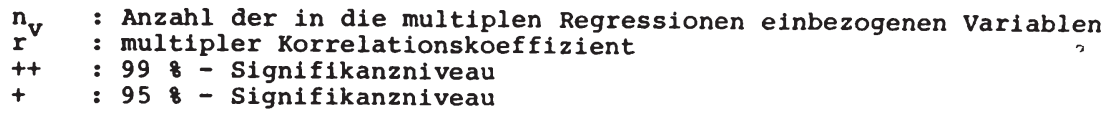


Bei den Aktivitäten im Bereich der Preisgestaltung festigen die Resultate die bei der Diskussion einzelner Rollenkomponenten aufgetretene Tendenz, nach der Handelsvertretungen der Investitionsgüterbranche ihrer Rolle bei einer eigenverantwortlichen Preis- und Konditionenfestlegung nur unzureichend gerecht werden können. Die Notwendigkeit einer nach Kundengruppen differenzierten Preisverantwortung wird damit erneut unter Beweis gestellt.

In ähnlicher Weise lassen sich für die produktanregungen parallele Resultate aufzeigen. Je größer die Bedeutung der Handelsvertretung für einen Abnehmer ist, d.h. je größer der vom Handelsvertreter bezogene Sortimentsanteil bzw. wertmäßige Einkaufsanteil ist, um so positiver wird die Rolle der Handelsvertretungen hinsichtlich der Mitwirkung bei den Herstellerangeboten eingeschätzt.

Einen hohen Grad der Rollenerfüllung verzeichnen dabei erstaunlicherweise auch jene Abnehmer, die den Verbundformen des Handels angehören. Wächst der Einkaufsanteil eines Kunden, den er über eine Einkaufszentrale abwickelt, wird die Rolle von Handelsvertretungen bei der Einflußnahme auf die Produktgestaltung der Hersteller gleichfalls positiv angesehen. Möglicherweise begünstigen die persönlichen Kontakte zwischen Handelsvertreter und Kunde - im Vergleich zu den eher unpersönlichen Beziehungen zur Einkaufszentrale - diese Beurteilung.

Für Handelsvertretungen implizieren diese Ergebnisse die Notwendigkeit, die Verkaufsbemühungen nicht nur auf lukrative Großkunden (z.B. Einkaufsverbände, Genossenschaften) zu beschränken, sondern zur sicherstellung eines kundenorientierten Angebotsprogramms auch die Anregungen der letzten Handelsstufe aufzugreifen und an die vertretenen 
Unternehmen weiterzuleiten ${ }^{1}$.

Schließlich hat die Untersuchung für den Grad der Rollenerfüllung von Handelsvertretungen in den Aktivitätsbereichen der innerbetrieblichen Leistungsfähigkeit sowie beim Vertretungsprogramm erneut den Einfluß der Bezugsquellenstruktur und der Art der Einkaufsentscheidung als Bestimmungsgrößen identifiziert. Damit belegen auch die Einschätzungen der Kunden über das AusmaB, in dem Handelsvertretungen in der Lage sind, ihre Rolle entsprechend den Erwartungen dieses Marktpartners auszufüllen, die Notwendigkeit eines differenzierten Einsatzes der Aktivitäten in den verschiedenen Dienstleistungsbereichen.

\subsection{Zusammenfassende Hypothesenbeurteilung horizontaler Rollenbeziehungen}

Die Diskussion empirischer Befunde zu den horizontalen Rollenbeziehungen auf Hersteller- und Kundenebene soll durch eine Beurteilung der theoriegestützten Ausgangshypothesen zusammengefabt werden. Abbildung 53 stellt die Befunde in den einzelnen Dienstleistungsbereichen einander gegenüber.

Ausgangspunkt der Uberlegungen zum Grad der Rollenerfüllung als Ausdruck horizontaler Rollenbeziehungen im Handelsvertreter-Marketing war der Gedanke einer empirischen, nachweisbaren Differenzierung zwischen der Beurteilung gegenwärtiger Rollenerfüllung und den Rollenerwartungen der Marktpartner. Diese Hypothese differenzierter Rollenkompo-

1 Diese grundsätzliche Motivation zur Befriedigung von Kundenbeđürfnissen belegen gleichfalls die Resultate einer aktuellen empirischen Untersuchung, vgl. Winer, L., Schiff, J.S., Industrial Salespeople's Views on Motivation, in: IMM 1980, S. $319 \mathrm{ff.}$. 


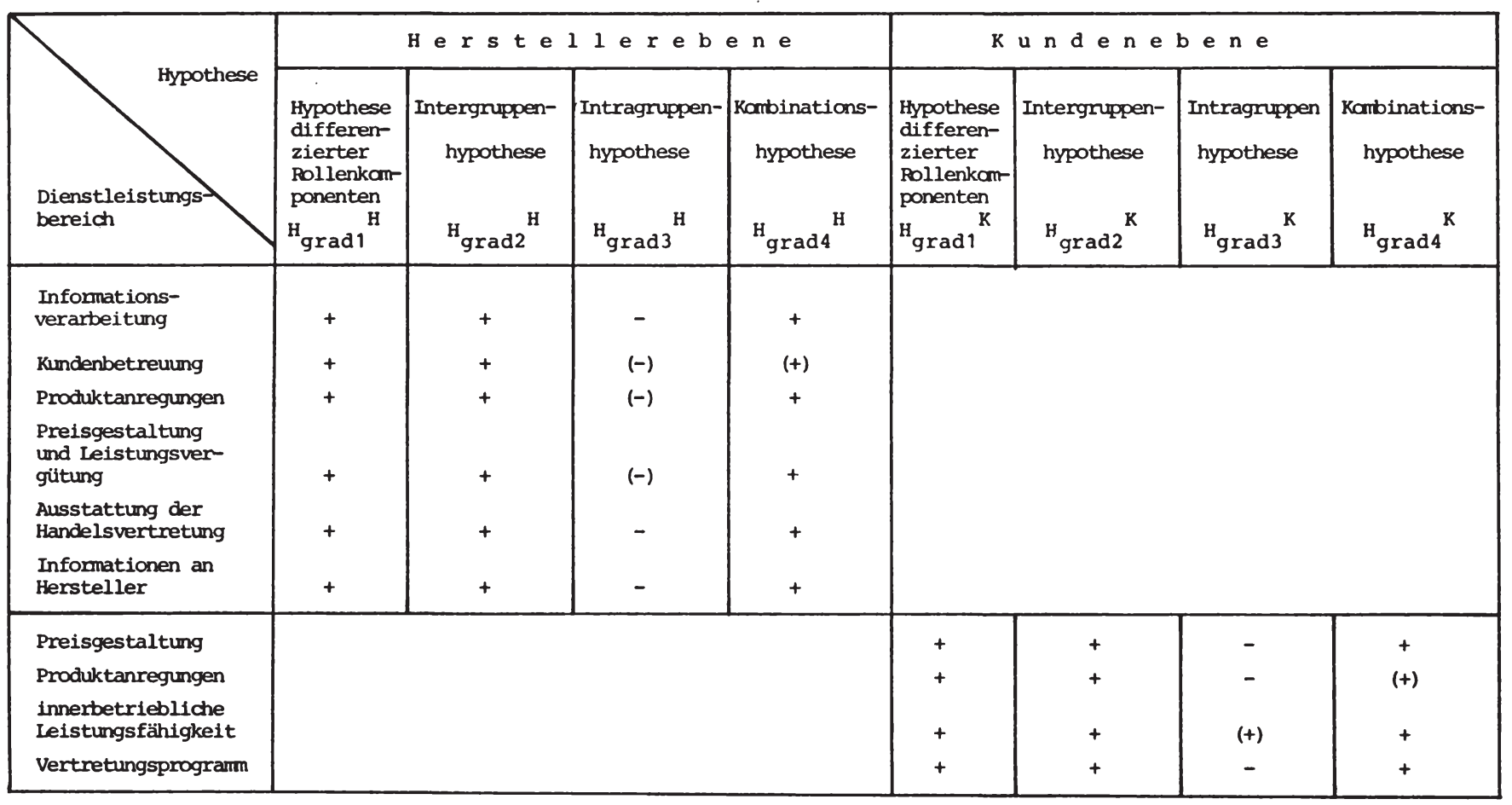

Abb. 53: Zusammenfassende Hypothesenbeurteilung horizontaler Rollenbeziehungen auf Hersteller- und Kundenebene

$+\quad$ : Hypothese bestätigt

- : Hypothese nicht bestätigt

(t) : Hypothese schwach bestätigt/abgelehnt 
nenten kann nach den vorliegenden empirischen Befunden für alle Dienstleistungsbereiche von Handelsvertretungen und beide Marktpartner als bestätigt gelten.

Der Sonderfall einer tbereinstimmung der beiden einzelnen Komponenten Rollenerfüllung und -erwartung konnte wertvolle Anhaltspunkte zur Relativierung einzelner Dienstleistungsbereiche im Handelsvertreter-Marketing bereitstellen. Dabei war es zugleich möglich, Unterschiede und Gemeinsamkeiten in den Beurteilungen von Herstellern und Abnehmern - und damit potentielle Konfliktbereiche transparent zu machen.

Weiterhin ist die Intergruppen-Hypothese für alle Leistungsbereiche anzunehmen, d.h. unternehmensbezogene Merkmale haben einen höheren EinfluB auf die Beurteilungen der Marktpartner als personale Merkmale der unmittelbaren Kontaktpartner von Handelsvertretern. Eine gewisse Relevanz personenbezogener Faktoren ist dennoch festzustellen, weil durch ihre Einbeziehung der alleinige Erklärungswert unternehmensdemographischer Merkmale gemäß den positiven Befunden zur Kombinationshypothese weiter erhöht werden konnte.

Als bemerkenswert ist schließlich festzuhalten, daß der in der Intragruppenhypothese angenommene dominierende EinfluB vertriebsbezogener Merkmale auf Herstellerebene bzw. einkaufsorientierter Faktoren bei den Kunden nicht generell nachgewiesen werden konnte. Lediglich einzelne Variablen der Vertriebs- oder Bezugsquellenstruktur sowie der Art der Einkaufsentscheidung konnten aussagefähige Interpretationen bereitstellen. 
Als wesentliches Resultat für eine Maßnahmenplanung im Handelsvertreter-Marketing ist schließlich die wiederholt deutlich gewordene Notwendigkeit einer differenzierten Ausgestaltung der Dienstleistungsbereiche im Hinblick auf unterschiedliche Zielgruppen sowohl auf Hersteller- als auch auf Kundenebene $z u$ betonen. Mögliche Anpassungen erfordern wegen der Heterogenität des gesamten Leistungsangebotes die Abgrenzung von Schwerpunkten, die vorrangig $\mathrm{zu}$ beachten sind. Eine Identifikation solcher Schwerpunkte wird mit einer Gegenüberstellung der wechselseitigen Vorstellungen und Meinungen der Marktpartner möglich. Diese Vorgehensweise führt entsprechend dem Grundmodell einer Rollenanalyse (vgl. Abbildung 9) zur Diskussion vertikaler Rollenbeziehungen im Handelsvertreter-Marketing.

\subsection{Vertikale Rollenbeziehungen}

Die duale Einbindung der Handelsvertretung in den Absatzkanal führt zu einer zweiseitigen Struktur vertikaler Rollenbeziehungen. Entsprechend der theoriegestützten Hypothesen $\mathrm{zu}$ diesem Untersuchungsbereich wird im folgenden zunächst die empirische Grundstruktur von Rollendissens und Rollendefizit vorgestellt, un auf dieser Grundlage die Beziehungen zwischen Handelsvertretungen und Herstellern bzw. Handelsvertretungen und Kunden in ihren empirischen Ausprägungen in den einzelnen Dienstleistungsbereichen $\mathrm{zu}$ beleuchten.

\subsection{Empirische Grundstruktur von Rollendissens und -defizit}

Die Analyse vertikaler Rollenbeziehungen zwischen Handelsvertretungen und ihren Marktpartnern erfordert eine integrative Darstellung der bereits einzeln im Detail diskutierten Rollenkomponenten Rollenausübung (aus Handels- 
vertretersicht) sowie Rollenerfüllung und -erwartung (aus Hersteller- bzw. Kundensicht). Im Hinblick auf die prognostische Relevanz für das Handelsvertreter-Marketing wird zur Veranschaulichung der Rollenbeziehungen jeweils auf die Dienstleistungsstruktur zurückgegriffen, die sich aus den Erwartungen der Hersteller abgeleitet hat ${ }^{1}$.

Abbildung 54 und 55 fassen jeweils die drei entsprechenden Rollenkomponenten zusammen und dokumentieren damit die empirische struktur vertikaler Beziehungen zwischen Handelsvertretungen und ihren Marktpartnern in allen Dienstleistungsbereichen ${ }^{2}$. Von den Beurteilungen der Hersteller und Kunden wird eine Bandbreite aufgespannt ${ }^{3}$; innerhalb dieser Bandbreite liegen - von wenigen Ausnahmen abgesehen die Einschätzungen der Handelsvertretungen über ihre Rollenausübung. Mit diesen empirischen Belegen findet somit das theoretisch entwickelte Grundmodell der Rollenanalyse im Handelsvertreter-Marketing eine empirische Bestätigung ${ }^{4}$.

1 Vgl. Kapitel C. 2.2 dieser Arbeit.

2 Die in der Abbildung eingetragenen Profile kennzeichnen die Mittelwerte der Antworten der Befragten. Eine verdichtete Darstellung der hier in einzelne Leistungsbereiche untergliederten Resultate geben Meffert, H., Kimmeskamp, G., a.a.O., S. $14 \mathrm{ff}$.

3 Diese Bandbreite - d.h. der Abstand zwischen Rollenerfüllung und -erwartung - ist letztlich eine andere Darstellungsform der zuvor diskutierten horizontalen Rollenbeziehungen.

4 Die Resultate über die Beziehungen zwischen Handelsvertretungen und Kunden erlauben gleichfalls diese Schlußfolgerung, vgl. Kapitel B. 4.22. 
$\mathrm{HOCH}$

NIEDRIG

KUNDENGE TREUUNG

QUALIFIZIERTE MITARBEITER AKTUALISIERTE RUNUENKAHTEI

KENNTNIS DER UMSATZKUNUE IVSTRUKTUR

LANGFRISTIGER KUPUENKOIVTAKT

EAWEITERUNG DES KUNUENKHEISES

HERSTELLER ERGAENZEN SICH GUT

\section{INF ORMATIONSAUSTAUSCH}

VERWERTUNG HERSTELLERIINFORMATIONEN VERWERTUNG KUNDENIINFORMATIONEN GENUEGEND INF ORMATIONEN AN KUNDEN HERSTELLERINFORMATION UEBER PLANUNGEN AUSREICHEND BERICHTE AN HERSTELLER

\section{PRODUKTANREGUINGEN}

BEE INFLUSSUNG HERSTELLEHSURTIMENT ANREGUNGEN FUEK NEUE PHUDUKTE VERTRE TUNGSPRUGRAMM NACH MARKTFORSCHUNG

\section{PRE ISGESTALTUNG}

ERMESSENSSPIELRAUM FUER KUNUITIONEN HANOLUNGSSPIELRAUM FUEK PREISNACHLAESSE GESONOERTE VERGUETUIVG LUSAETZL. LEISTUNG

AUSSTATTUNG DER HANUELSVERTRETUNG

BUERO PERSONELL BESETZT

BUERO HAT TECHN. HILFSMITTEL

\section{LEISTUNGSVERGUETUNG}

KALKULATION PROVISIONSSAETZE PROVISIONSVORSCHLAG

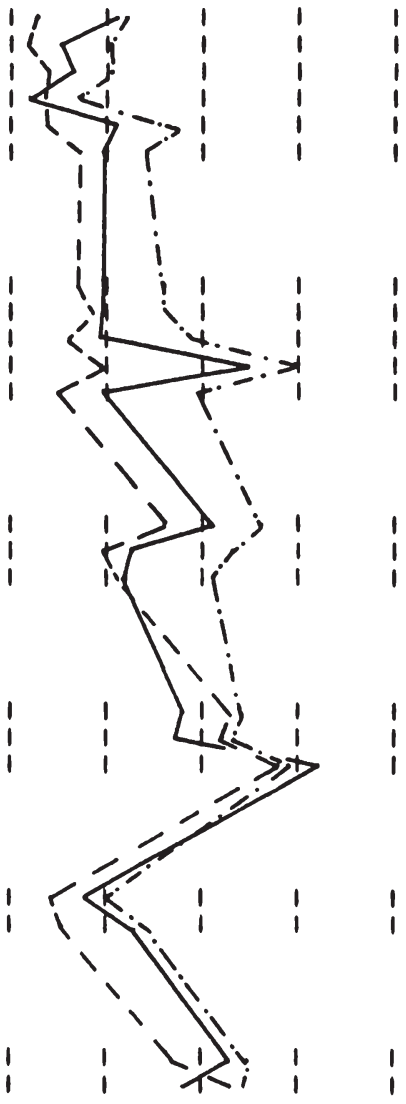

AHB. 54: ROLLENREZIEHUNGEN ZWISCHEN HAINUELSVERTRETUINGEN UNO VERTRETENEIV UINTERNEHAEN

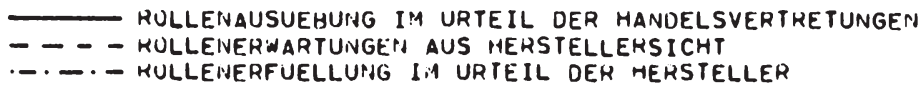




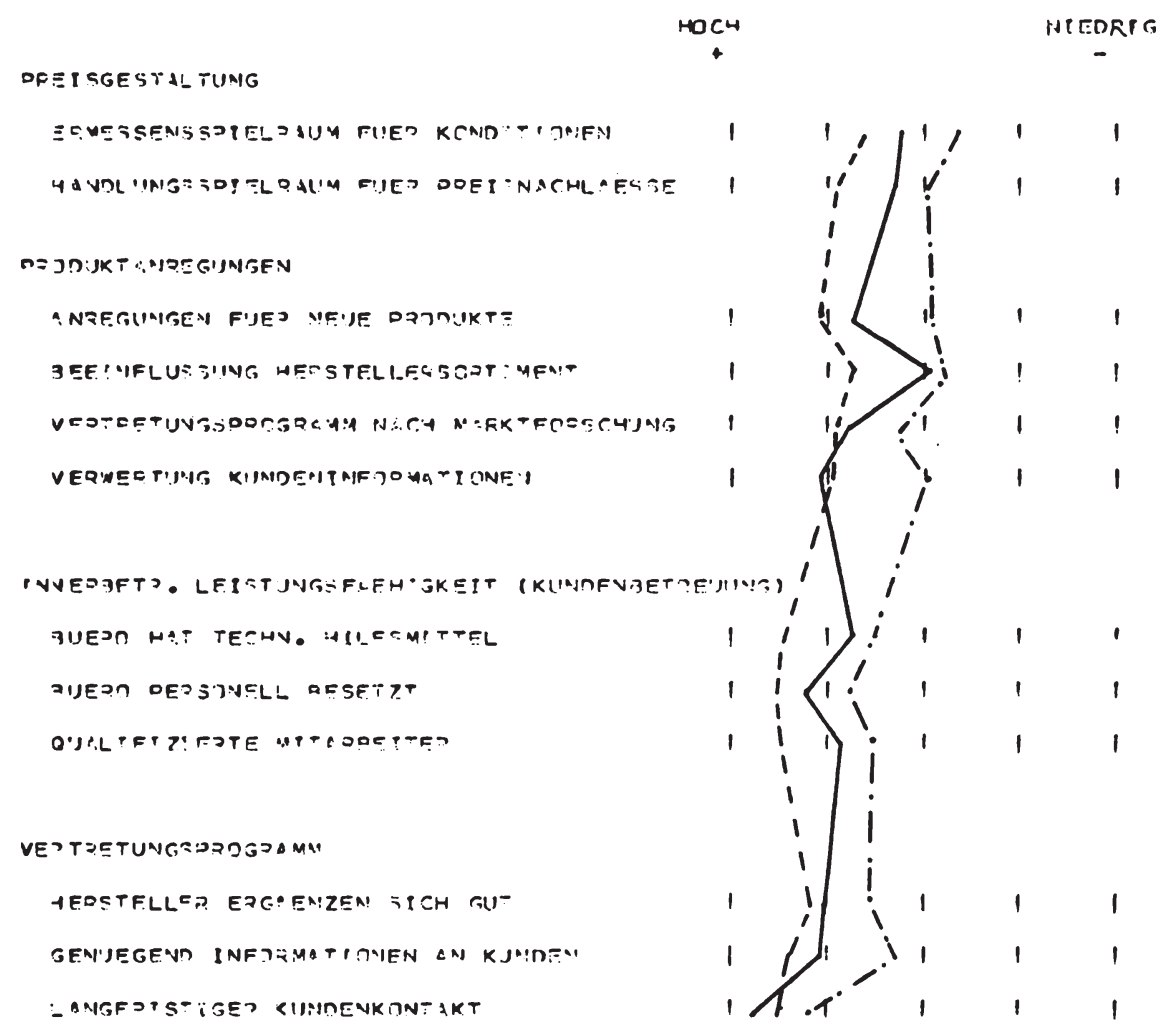

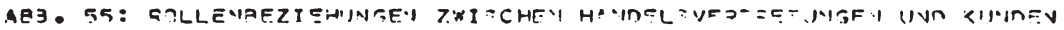

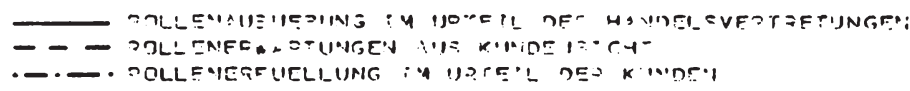


Die Beurteilungen der vertretenen Unternehmen und Kunden über die Rollenerfüllung von Handelsvertretungen (rechtes Profil) liegen in fast allen Leistungsbereichen unterhalb der Selbsteinschätzung der Rollenausübung durch die Handelsvertreter (mittleres Profil). Der Abstand dieser beiden Profile kennzeichnet das Ausmaß an Rollendefizit zwischen den Marktpartnern. Ein Vergleich der Rollenausübung mit den Rollenerwartungen der Hersteller und Kunden (linkes Profil) deckt bei allen Leistungsmerkmalen auf, wo ein Rollendissens zwischen Handelsvertretungen und ihren Marktpartnern besteht. Dissens und Defizit bei der Beurteilung der Rolle von Handelsvertretungen im vertikalen Marketing beschreiben Bereiche potentieller Konflikte zwischen den Marktpartnern ${ }^{1}$.

Für das Handelsvertreter-Marketing stellt sich die Frage, welche Konsequenzen diese Resultate zur Folge haben. Ausgehend von der unterschiedlichen Beurteilung des statusquo durch Handelsvertreter und Hersteller bzw. Kunden erscheint es sinnvoll, zunächst Divergenzen in der Einschätzung des gegenwärtigen Leistungsangebotes $\mathrm{zu}$ beseitigen, um auf dieser neuen - gemeinsamen - Grundlage über Leistungsanpassungen entscheiden zu können. Der Abbau von Rollendefizit bildet damit den ersten Schritt zur Harmonisierung der Beziehungen im vertikalen Marketing.

Der Abbau von Rollendefizit zwischen Handelsvertretungen und Herstellern/Kunden kann nur unter Berücksichtigung der Ursachen der divergierenden Beurteilungen vorgenommen werden. Obwohl die empirische Untersuchung diese Frage nicht explizit beantworten kann, lassen die Untersuchungsergebnisse mögliche Schlußfolgerungen erkennen. So ist beispiels-

1 Vgl. hierzu die Ausführungen in Kapitel B. 1.33. 
weise die positivere Selbsteinschätzung der Rollenausübung durch die Handelsvertretungen - im Vergleich zur Beurteilung ihrer Rollenerfüllung durch vertretene Unternehmen und Abnehmer - neben einem ausgeprägten Selbstbewußtsein auf einen unterschiedlichen Informationsstand der Marktpartner zurückzuführen.

Bisherige Resultate sowie Erfahrungen aus Voruntersuchungen lassen die Annahme $z u$, daß die Kunden und insbesondere die Hersteller nicht immer über das tatsächliche Leistungsangebot der Handelsvertretungen informiert sind ${ }^{1}$. Fehlende Informationen über Leistungen generell bzw. die Intensität bestimmter Leistungen führen zwangsläufig $\mathrm{zu}$ der als Rollendefizit beschriebenen Diskrepanz zwischen Handelsvertretungen und Marktpartnern. Gelingt es den Handelsvertretungen, ihr Dienstleistungsangebot in allen Bereichen transparenter zu machen, läßt sich eher eine übereinstimmende Beurteilung herbeiführen und damit das Rollendefizit erheblich vermindern.

Eine zweite Konsequenz vorliegender empirischer Resultate gilt dem Abbau von Rollendissens. Die Untersuchung belegt in fast allen Leistungsbereichen Erwartungen an die Rolle von Handelsvertretungen, die über ihre bisherige Rollenausübung hinausgehen. Handelsvertreter sind damit aufgefordert, ihr Leistungsangebot im Hinblick auf eine mögliche Anpassung $\mathrm{zu}$ überprüfen. Eine Modifikation bisheriger Dienstleistungen mu dabei den bereits herausgearbeiteten Anforderungen unterschiedlicher Zielgruppen auf seiten der Marktpartner in besonderem Maße genügen. Weiterhin gilt es, die kostenmäßigen Konsequenzen von Leistungsanpassungen unter Einbeziehung der durch eine Veränderung zu er-

$1 \mathrm{Vgl}$. Meffert, H., Die Information ist verbesserungsfähig, a.a.O., S. 1440 . 
wartenden Auftragszunahme zu kalkulieren, um die Wirtschaftlichkeit derartiger Maßnahmen abschätzen zu können. Konkrete Ansatzpunkte für das Handelsvertreter-Marketing stellen dazu die Resultate einzelner Dienstleistungsbereiche im Detail bereit.

4.22 Rollenbeziehungen zwischen Handelsvertretungen und ihren Marktpartnern in einzelnen Dienstleistungsbereichen

\subsection{Beziehungen zwischen Handelsvertretungen und Her- stellern}

Bei der Kundenbetreuung (vgl. Abbildung 54) liegen die Handelsvertretungen mit der Einschätzung ihrer Rollenausübung in nahezu allen Bereichen in der durch die Rollenerfüllung und Rollenerwartung der Hersteller aufgespannten Bandbreite. Rollendissens und-defizit sind bei der Einschätzung der Mitarbeiterqualifikation nahezu gleich groß, d.h. die Beurteilungen der Handelsvertretungen liegen genau mitten zwischen den Herstellermeinungen. Vier weitere Merkmale weisen dagegen ein Rollendefizit aus, das durchschnittlich doppelt so hoch ist wie der entsprechende Rollendissens (Kundenkartei und-struktur, Gewinnung neuer Kunden, Abrundung des Vertretungsprogramms). Da der Abbau von Rollendefizit tendenziell weniger konfliktär ist als beim Rollendissens, läßt sich die Position der Handelsvertretung in den genannten Bereichen durch geeignete informationspolitische Maßnahmen entscheidend verbessern.

In der Beurteilung der Langfristigkeit der Kundenbeziehungen liegt die Einschätzung der Rollenausübung außerhalb der von den Herstellerbeurteilungen aufgespannten Bandbreite. Handelsvertreter sehen hier offensichtlich eine stärke ihrer 
eigenen Leistungsfähigkeit, die jedoch von den vertretenen Unternehmen nicht in diesem hohen Maße wahrgenommen wird. Gleichzeitig liegen die Erwartungen der Hersteller unterhalb der von den Handelsvertretungen angegebenen Rollenausübung. Insgesamt wird jedoch die Langfristigkeit der Kundenbeziehungen von den Herstellern anerkannt; im Vergleich zu allen übrigen Dienstleistungsmerkmalen liegt hier die am höchsten positive Beurteilung durch die vertretenen Unternehmen. Die erkennbare Neigung von Handelsvertretern zur Uberschätzung ihrer Position in Bezug auf die langfristigen Kundenbeziehungen sollte kritisch hinterfragt und zumindest nicht ausgebaut werden.

Betrachtet man den Informationsaustausch im Handelsvertreter-Marketing, setzt sich die bei der Kundenbetreuung diskutierte Grundrichtung der empirischen Resultate weiter fort. Das Rollendefizit ist vielfach erheblich größer als der Rollendissens, wobei eine Polarisierung zwischen hersteller- und kundenbezogenen Informationen besteht. Bei den herstellerorientierten Informationsaktivitäten (Berichte an Hersteller, Verwertung von Herstellerinformationen) ist das Ausmaß des Rollendefizites tendenziell doppelt so hoch wie der Rollendissens; bei den kundenbezogenen Informationsaktivitäten (Information an Kunden, Verwertung der Kundeninformation) ist das Rollendefizit weitaus höher als der jeweilige Rollendissens. Dieses Resultat verlangt eine unmittelbare Berücksichtigung in den Aktivitäten von Handelsvertretungen gegenüber ihren vertretenen Unternehmen. Der hier in der Tendenz erkennbare Vorwurf einer mangelnden Kundenorientierung im Informationsverhalten ist durch eine entsprechende Bewußtmachung der tatsächlichen Leistungsfähigkeit von Handelsvertretungen zu relativieren. 
Eine weitere Profilierungsmöglichkeit belegen die Ergebnisse zu der Frage, in welchem Ausmaß Handelsvertretungen die Hersteller über ihre eigenen Planungen und $\mathrm{Ziel-}$ setzungen informieren. Hier liegen Rollenausübung und Rollenerfüllung relativ nahe zusammen, d.h. das Rollendefizit ist nur gering ausgeprägt. Die Rollenerwartungen der Hersteller liegen auf einem erheblich höheren Niveau und spannen damit einen umfassenden Rollendissens auf. Die hoch angesiedelten Erwartungen der vertretenen Unternehmen bieten für Handelsvertretungen die Chance, die Zusammenarbeit mit den Herstellern durch eine Intensivierung der Information über eigene unternehmenspolitische Zielsetzungen (z.B. Jahresplanungen) besser abzustimmen. Verbesserungen der beschriebenenArt festigen dabei die Position von Handelsvertretung und Hersteller durch ein beiderseits koordiniertes Vorgehen am Markt in gleichem Maße.

Im Leistungsbereich der Produktanregungen sind die Beziehungen bei der Einflußnahme von Handelsvertretungen auf das Herstellersortiment durch Rollendissens und-defizit in annähernd gleichem AusmaB gekennzeichnet. Ein erheblich größeres Rollendefizit verzeichnen dagegen die Einschätzungen über die Vermittlung von Anregungen zur Neuproduktentwicklung sowie zur Zusammensetzung des Vertretungsprogramms der Handelsvertretungen. Die Aktivitäten der Handelsvertreter werden in beiden Bereichen von den vertretenen Unternehmen weitaus negativer eingeschätzt als von den Handelsvertretern selbst.

Hinsichtlich der Neuproduktanregungen bietet es sich für Handelsvertretungen demnach an, den konkreten Informationsbedarf der Hersteller in Erfahrung zu bringen, um die eigenen Leistungen besser darauf abzustimmen. So ist es bei- 
spielsweise wenig sinnvoll, den vertretenen Unternehmen nur globale Markttrends mitzuteilen, wenn vorrangig Detailinformationen der Produktnutzung zur technischen Produktverbesserung benötigt werden. In ähnlicher Weise kann das Rollendefizit beim Vertretungsprogramm abgebaut werden, wenn die Handelsvertreter den vertretenen Unternehmen die Kriterien der Zusammenstellung der Vertretungen transparent machen, wobei die Berücksichtigung von Hersteller- und Kundeninteressen gleichermaßen deutlich werden muß.

Im Bereich der Preisgestaltung lassen sich Rollendissens und-defizit der bisher beschriebenen Form nicht empirisch nachweisen. Die Studie belegt vielmehr erhebliche Meinungsunterschiede zwischen Handelsvertretungen und vertretenen Unternehmen bei den drei betrachteten Einzelmerkmalen. Demnach sehen die Handelsvertreter den ihnen zur Verfügung stehenden preis- und konditionenpolitischen Handlungsspielraum sehr viel positiver, als dieser durch die Hersteller eingeschätzt wird.

Zugleich hat sich zwischen der Beurteilung der Rollenerfüllung und den Rollenerwartungen der Hersteller nur eine relativ geringe Diskrepanz herausgestellt, d.h. die Handelsvertretungen genügen - aus Herstellersicht - bereits annähernd den Erwartungen.

Die Bewertungsunterschiede zwischen Handelsvertretungen und Herstellern belegen erneut die Subjektivität der Einschätzung von Sachverhalten, die bei objektiver Betrachtung $\mathrm{zu}$ einer identischen Beurteilung führen müßten. Für das Handelsvertreter-Marketing belegen die Resultate somit das Erfordernis einer behutsamen Vorgehensweise bei einer weiteren Einflußnahme auf die Preis- und Konditionenverantwortung. Die unterschiedliche Einschätzung quantitativ 
gleicher Preis- und Konditionenspannen stellt damit zugleich die Notwendigkeit und Tragfähigkeit des rollenorientierten Ansatzes zur Einbeziehung subjektiver Vorstellungen der Marktpartner unter Beweis.

Bei der gesonderten Vergütung zusätzlicher Leistungen haben sich inhaltlich entgegengesetzte Resultate herausgestellt. Hier liegt die Einschätzung der Rollenausübung erheblich unter der Rollenerfüllung sowie der Rollenerwartung. Handelsvertretungen sehen demnach die Vergütung von zusatzleistungen in weitaus geringerem Maße realisiert als ihre vertretenen Unternehmen. Die Stärke der Abweichung zwischen den Vorstellungen von Handelsvertretungen und Herstellern belegt erneut die umstrittene Diskussion über eine derartige Kostenverteilung. Eine entsprechende Kalkulation zusätzlicher Leistungen hinsichtlich ihrer Kostenwirksamkeit kann dabei in Verhandlungen mit den vertretenen Unternehmen als wirksame Entscheidungshilfe dienen.

In ähnlicher Weise können Handelsvertretungen durch eine verstärkte Kalkulation ihrer Provisionssätze im Rahmen der Leistungsvergütung dazu beitragen, den Rollendissens bei diesem Leistungsmerkmal abzubauen. Die Studie belegt ein nur gering ausgeprägtes Rollendefizit, d.h. die Beurteilungen von Handelsvertretungen und Herstellern über die gegenwärtige Situation bei der Provisionskalkulation weisen hohe tbereinstimmungen auf. Demgegenüber eröffnet ein mehr als doppelt so großer Rollendissens die beschriebene Chance für Handelsvertretungen. Bei allen Aktivitäten im Bereich der Leistungsvergütung ist jedoch $z u$ beachten, da $\beta$ die Erwartungen der vertretenen Unternehmen bei der Frage nach dem Initiator von Provisionsvorschlägen erheblich unter der von den Handelsvertretungen wahrgenommenen Rollenausübung liegen. Das hier erkennbare Konflikt- 
risiko ist in jedem Falle bei der Maßnahmenplanung $z u$ berücksichtigen.

Die Ausstattung der Handelsvertretung in materieller und personeller Hinsicht wird von den Handelsvertretern selbst und den vertretenen Unternehmen übereinstimmend als relativ positiv angesehen. Ein Rollendefizit ist demnach in beiden Einzelmerkmalen nur in geringem Maße anzutreffen. Rollendissens besteht dagegen in der Frage über das anzustrebende Aktivitätsniveau. Die hohen Erwartungen der Hersteller bieten grundsätzlich die Möglichkeit einer Leistungsintensivierung; insbesondere die technische Ausstattung der Handelsvertretung weist einen hohen Rollendissens auf. Auch an dieser Stelle ist eine Leistungsverbesserung nicht ohne entsprechende Wirtschaftlichkeitsüberlegungen sinnvoll. Gleichzeitig sollten alle Maßnahmen nur in Koordination mit den Vorstellungen und Erwartungen der Abnehmer erfolgen.

\subsection{Beziehungen zwischen Handelsvertretungen und Kunden}

Betrachtet man im Rahmen der Beziehungen zwischen Handelsvertretungen und Kunden den Bereich der Preisgestaltung, erfahren bisherige Resultate eine weitgehende Bestätigung. Die Gegenüberstellung der Vorstellungen der Marktpartner deckt dabei eine günstigere Beurteilung der Konditionenpolitik im Vergleich auf. Rollendissens und -defizit treten bei den Konditionen in gleichem Maße auf; der Ermessensspielraum für Preisnachlässe beinhaltet dagegen einen wesentlich höheren Rollendissens.

Der Rollendissens zwischen Handelsvertretungen und Kunden bei der Preisgestaltung ist für das HandelsvertreterMarketing in besonderem Maße problematisch, weil die Er- 
wartungen der Hersteller hinsichtlich der Preisverantwortlichkeit von Handelsvertretungen erheblich niedriger liegen. Bei der Festlegung des preispolitischen Handlungsspielraumes müssen daher Handelsvertretung und Hersteller einen Kompromi $\beta$ finden zwischen den Interessen des vertretenen Unternehmens (geringe Handlungsspielräume) und der Notwendigkeit einer flexiblen Preisgestaltung am Markt (höhere Handlungsspielräume).

Bei den Produktanregungen - zu denen die Kunden ferner einige informationsbezogene Aktivitäten rechnen - ist es den Handelsvertretungen nicht bei allen einzelnen Merkmalen gelungen, die Kunden von ihrer Leistungsfähigkeit $z u$ überzeugen. Dies gilt besonders für die Weiterleitung von Anregungen an die Hersteller zur Neuproduktplanung und für die Zusammensetzung des Vertretungsprogramms; beide Bereiche weisen ein hohes MaB an Rollendefizit auf. Bedenklich für Handelsvertretungen ist vor allem der Vorwurf der Kunden, das Vertretungsangebot der Handelsvertretungen werde nur wenig nach marktbezogenen Erfordernissen zusammengestellt. Eine Intensivierung der Bemühungen in diesem Bereich eröffnet den Handelsvertretern aufgrund ihrer Position als selbständige Absatzmittler in besonderem Maße eine wirkungsvolle Abgrenzung von den Reisenden der Hersteller.

Ein hoher Rollendissens besteht hinsichtlich der EinfluBnahme von Handelsvertretungen auf das Sortiment der Hersteller. Die Beurteilung der Rollenerfüllung durch die Kunden sowie die Rollenausübung weichen wenig voneinander ab; das Rollendefizit ist demnach weitaus geringer. Anpassungen des Leistungsniveaus an die Erwartungen der Kunden sind dabei relativ unproblematisch, weil die Erwartungen der Hersteller in dieselbe Richtung zielten. 
Einen Schwachpunkt der Handelsvertretertätigkeit belegen die empirischen Resultate zur Verwertung von Kundeninformationen. Hier fällt die Rollenausübung der Handelsvertretungen aus der von Rollenerfüllung und -erwartung aufgespannten Bandbreite heraus. Handelsvertreter schätzen ihre Leistungen weit positiver ein, als sie von den Kunden gesehen werden; sie übersteigen sogar die Erwartungen der Kunden. Offenbar glauben die Kunden nicht, daß Handelsvertreter die beim Besuch ausgetauschten Informationen in adäquater Form verarbeiten (z.B. hinsichtlich differenzierter Branchenkenntnisse), während die Handelsvertreter selbst in wesentlich höherem Maße Kundeninformationen sammeln und aufbereiten. Auch in diesem Bereich gilt es, den Kunden die Leistungsfähigkeit von Handelsvertretungen verstärkt deutlich zu machen. Parallele Resultate auf Herstellerebene begünstigen dabei die Chance zur Profilierung als 'Informationsrelais' im Absatzkanal.

Die innerbetriebliche Leistungsfähigkeit wird von den Kunden - ähnlich wie zuvor von den vertretenen Unternehmen positiv eingeschätzt. In gleicher Weise wie auf der Herstellerebene belegt die technische Ausstattung der Handelsvertretung einen hohen Rollendissens. Offensichtlich gehen die Erwartungen von Herstellern und Kunden an moderne Kommunikationsmittel über das derzeit verfügbare Angebot erheblich hinaus. Diese gleichlautenden Resultate beider Marktpartner sollten Handelsvertretungen zum AnlaB einer kritischen Uberprüfung ihrer Leistungsfähigkeit in diesem Bereich nehmen.

Einer Uberprüfung bedarf gleichfalls die positive Einschätzung der Wirkung des Vertretungsprogramms auf die Kunden. Insbesondere die von den Handelsvertretungen betonte langfristigkeit in den Kundenbeziehungen wird von den 
Abnehmern selbst niedriger eingeschätzt; auch die Erwartungen an die Rolle von Handelsvertretungen liegen noch unterhalb der Rollenausübung. Als besonders bemerkenswert ist hervorzuheben, daß die Beurteilungen von Herstellern und Kunden nahezu völlig übereinstimmen. Die positive Hervorhebung der Rollenausübung bei der langfristigen Zusammenarbeit mit den Kunden muß angesichts dieser Resultate relativiert werden. Gleichwohl ist eine Leistungsreduzierung noch nicht angezeigt, da die empirischen Befunde an dieser Stelle keine vergleichende Aussage über eine entsprechende Beurteilung von unternehmensangestellten Reisenden ermöglichen.

\subsection{Zusammenfassende Hypothesenbeurteilung vertikaler Rollenbeziehungen}

Die für die einzelnen Leistungsmerkmale von Handelsvertretungen vorgestellten Befunde vertikaler Rollenbeziehungen im Handelsvertreter-Marketing lassen sich durch eine Beurteilung der Ausgangshypothesen abschließend zusammenfassen. Abbildung 56 stellt die in den einzelnen Dienstleistungsbereichen herausgearbeiteten Resultate den Hypothesen gegenüber ${ }^{1}$.

Die über das erwartete Defizit in den Beziehungen der Marktpartner formulierte Hypothese erfährt durch die empirische Studie sowohl in den Beziehungen zwischen Handelsvertretungen und vertretenen Unternehmen als auch zwischen Handelsvertretungen und Kunden eine Bestätigung. Diese Rollendefizithypothese gibt damit den Handelsvertretungen $A n l a B$, ihre Rollenausübung im Spannungsfeld von Industrie

$1 \mathrm{Vgl}$. Kapitel B. 3.3 sowie insbesondere Abbildung 9. 


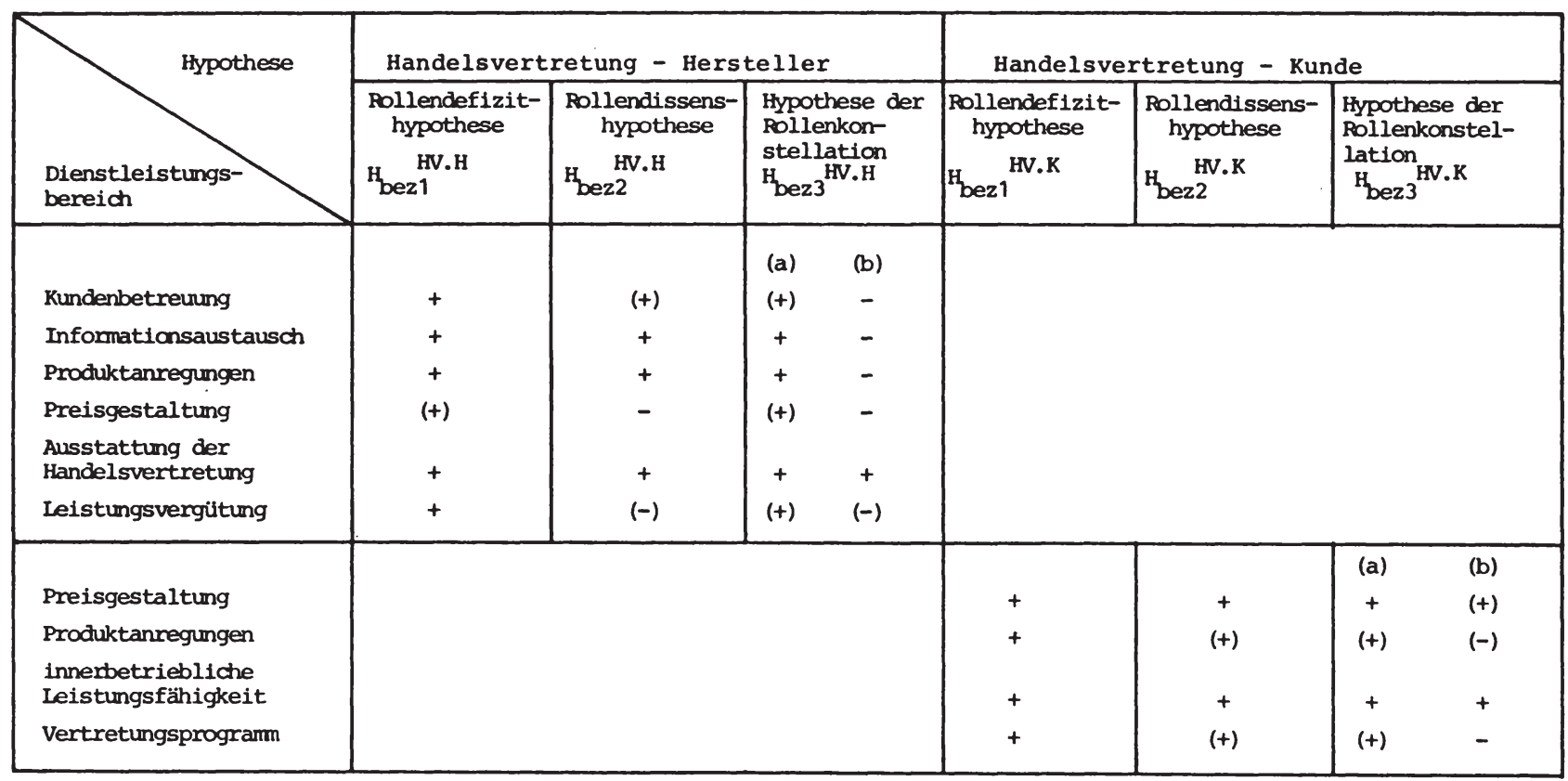

Abb. 56: Zusammenfassende Hypothesenbeurteilung vertikaler Rollenbeziehungen

$+\quad$ : Hypothese bestätigt

- : Hypothese nicht bestätigt

$( \pm)$ : Hypothese schwach bestätigt/abgelehnt 
und Handel kritisch $\mathrm{zu}$ überdenken. Die in allen Dienstleistungsbereichen aufgetretenen Abweichungen zwischen der Beurteilung der Rollenerfüllung durch die Marktpartner und der Einschätzung der Rollenausübung sind weiterhin durch geeignete Informationsaktivitäten abzubauen. Die Beseitigung von Rollendefizit - d.h. der Unkenntnis der Marktpartner über das Leistungsangebot hinsichtlich Umfang und Intensität - schafft die Voraussetzung zur Ubberprüfung des Dienstleistungsangebotes im Hinblick auf mögliche Anpassungen an die Rollenerwartungen der Marktpartner.

Erwartungsgemäß bestätigen die empirischen Resultate auch die Rollendissenshypothese. Mit einer Ausnahme bei der Preispolitik liegen damit die Rollenerwartungen der Marktpartner in allen Dienstleistungsbereichen höher als die Rollenausübung aus der Sicht der Handelsvertreter. Das für einzelne Leistungsmerkmale unterschiedlich hohe Ausma $B$ an Rollendissens hat zugleich die Notwendigkeit eines differenzierten Vorgehens bei einer beabsichtigten Anpassung im Leistungsangebot unter Beweis gestellt.

In manchen Leistungsbereichen ist eine derartige Anpassung dabei relativ problemlos möglich, weil die Interessen der Marktpartner von Handelsvertretungen in dieselbe Richtung zielen (z.B. Produktanregungen, technische Ausstattung der Handelsvertretung). Andere Leistungsbereiche fordern dagegen in weit höherem Maße die Vermittlungsfähigkeit von Handelsvertretern zum Ausgleich divergierender Interessen von vertretenen Unternehmen und Abnehmern (z.B. bei der Preisverantwortung).

Aus den Resultaten zur Rollendissens- und defizithypothese leiten sich die Aussagen zur Hypothese der Rollenkonstellation unmittelbar ab. Abgesehen von den im Detail diskutierten Ausnahmen liegt die Rollenausübung der Handelsvertre- 
tungen in allen Dienstleistungsbereichen (konkreter: bei den einzelnen Leistungsmerkmalen) zwischen der Rollenerfüllung und den Rollenerwartungen der Marktpartner (Spalten a in Abbildung 56).

Der vermutete Zusammenhang eines größeren Rollendissens im Vergleich zum Rollendefizit läßt sich nur für die Ausstattung der Handelsvertretung in den Beziehungen zu beiden Marktpartnern eindeutig bestätigen (Spalten b in Abbildung 56). Die meisten übrigen Dienstleistungsbereiche weisen dagegen ein höheres Rollendefizit auf. Angesichts dieser Konstellation gewinnen die Aussagen an Bedeutung, die bei der Diskussion einzelner Befunde zur Beseitigung von Rollendefizit formuliert wurden. Die Beziehungen zwischen Handelsvertretungen und ihren Marktpartnern sind damit letztlich weniger durch konfliktäre Rollendissense geprägt als vielmehr durch eine unterschiedliche Beurteilung von angebotenen Leistungen (Rollenausübung) und der Wahrnehmung dieser Leistungen durch die Marktpartner (Rollenerfüllung).

Ansatzpunkte zur Verbesserung der Position von Handelsvertretungen im Absatzkanal haben sich vielversprechend mehrfach aufgezeigt. Aufgabe von Handelsvertretungen muß es sein, die entsprechenden Maßnahmen zur Realisierung eines Handelsvertreter-Marketing zu ergreifen. Ausgangspunkt aller Uberlegungen ist dazu die wiederholt empirisch belegte Notwendigkeit einer Ausrichtung an den jeweiligen Bedürfnissen unterschiedlicher Hersteller- und Kundengruppen. 
Im folgenden wird dazu auf der Basis der empirischen Studie untersucht, ob sich auf der Grundlage der rollenbezogenen Vorstellungen und Meinungen der Befragten eine entsprechende Segmentierung des Hersteller- und Kundenmarktes vornehmen läßt, die sich als Ausgangspunkt der Maßnahmenplanung eignet.

5. Rollenbezogene Segmentierung der Marktpartner als Grundlage eines differenzierten Handelsvertreter-Marketing

Die bisherige Analyse der Rolle von Handelsvertretungen im Absatzkanal hat wiederholt heterogene Vorstellungen der Marktpartner in Bezug auf das Dienstleistungsangebot von Handelsvertretungen aufgedeckt. Damit wurde zugleich die Notwendigkeit unter Beweis gestellt, die Planung von Maßnahmen im Handelsvertreter-Marketing an den unterschiedlichen Bedürnissen verschiedener Gruppen auf Herstellerund Kundenebene zu orientieren. Es ist daher erforderlich, sowohl die vertretenen Unternehmen als auch den Abnehmerkreis von Handelsvertretungen in geeigneter Form in homogene Teilgesamtheiten aufzugliedern, die sich jeweils als zielgruppe eines darauf abgestimmten Maßnahmenkataloges eignen.

Im folgenden werden auf der Basis der empirischen Untersuchung zunächst Ansatzpunkte einer derartigen Gruppierung auf Hersteller- und Kundenebene herausgearbeitet. Auf dieser Grundlage erfolgt eine entsprechende Typologisierung der Marktpartner von Handelsvertretungen gemäß der theoriegestützten Hypothesen zur rollenbezogenen Segmentierung. 
5.1 Ansatzpunkte und Vorgehensweise einer psychographischen Segmentierung im Rahmen der Rollenanalyse

Strategien zur Markterfassung im Handelsvertreter-Marketing müssen entsprechend der dualistischen Einbindung von Handelsvertretungen in den Absatzkanal beide Marktseiten als Ausgangspunkt einer Aufgliederung in homogene Teilmärkte berücksichtigen. Identifikation und Abgrenzung derartiger Segmente stellen die Basis für eine darauf aufbauende Marktbearbeitungsstrategie, wobei nach den bisherigen empirischen Befunden eine differenzierte Marktbearbeitung für Handelsvertreter unerläßlich ist ${ }^{1}$.

Ein differenziertes Agieren am Markt erfordert die Auswahl geeigneter Kriterien zur Abgrenzung der entsprechenden Segmente. Ausgehend von der generellen voraussetzung einer Aufgliederung des Gesamtmarktes in homogene Teilmärkte, die untereinander weitgehend heterogen sein sollen, haben die Segmentierungskriterien besonderen Anforderungen $\mathrm{zu}$ genügen $^{2}$. Demnach müssen sie für eine Berücksichtigung im Handelsvertreter-Marketing

- mit dem Dienstleistungsangebot von Handelsvertretungen sowie den Beurteilungen der Marktpartner in Verbindung stehen,

- mit vorhandenen Methoden der Marktforschung meßbar sein,

1 Zum Grundgedanken der Markterfassung und Marktbearbeitung vgl. Meffert, H., Marketing, a.a.O., S. 213 ff.; Frank, R.E., Massy, W.F., Wind, Y., Market Segmentation, a.a.O., S. $11 \mathrm{ff.;}$ Böhler, H., Methoden und Modelle der Marktsegmentierung, Stuttgart 1977, S. $10 \mathrm{f} .$.

2 Vgl. Meffert, H., Marketing, a.a.O., S. 214 f.; Kotler, P., Marketing-Management, a.a.O., S. 164 f.. 
- ausreichend große Segmente gewährleisten und

- über einen längeren zeitraum aussagefähig bleiben.

Aus der empirisch unterstützten Rollenanalyse im Handelsvertreter-Marketing erfüllen vor allem die Rollenerwartungen der Marktpartner die genannten Anforderungen und eignen sich daher als segmentierungskriterium ${ }^{1}$. Der Bezug zum Dienstleistungsangebot von Handelsvertretungen ist unmittelbar gegeben; aufgrund ihrer prognostischen Relevanz stellen Rollenerwartungen zudem eine längerfristige Aussagefähigkeit sicher.

Die allgemeine Aufgabenstellung einer Segmentierung von Hersteller- und Kundenmarkt läßt sich somit präzisieren. Gesucht wird eine Aufteilung beider Marktpartner von Handelsvertretungen, wobei sich die abgegrenzten Segmente durch ein hohes $\mathrm{MaB}$ an Ubereinstimmung hinsichtlich der Erwartungen an das Dienstleistungsangebot der Handelsvertretungen auszeichnen. Die Segmente unter einander sollen möglichst heterogene Erwartungshaltungen aufweisen. Gelingt die Abgrenzung derartiger Gruppierungen auf Herstellerund Kundenebene, lassen sich unmittelbar Konsequenzen für die Maßnahmenplanung im Handelsvertreter-Marketing ableiten.

Das methodische Instrumentarium zur Lösung dieser Aufgabenstellung stellen die Verfahren der Clusteranalyse bereit. Für praktische Segmentierungsprobleme finden zumeist heuristische Iterationsverfahren Anwendung, die zur Segment-

1 Im Rahmen der 'klassischen' Segmentierungskriterien sind Rollenerwartungen den psychographischen Kriterien zuzuordnen; vgl. etwa Böhler, H., a.a.O., S. 84 . 
bildung globale Gütekriterien verwenden (z.B. Minimierung der Varianzen innerhalb der gebildeten Gruppen) ${ }^{1}$.

Die Vorgehensweise der Clusterbildung und -beschreibung für die vorliegende Problemstellung erfolgt in drei stufen:

1. Segmentierung des Hersteller- und Kundenmarktes nach der Homogenität der Rollenerwartungen ${ }^{2}$.

2. Auswahl der für das Handelsvertreter-Marketing geeigneten clusterzahl ${ }^{3}$.

1 Vgl. Böhler, H., a.a.O., S. 297 ff.; Frank, R.E., Green, P.E., Numerical Taxonomy in Marketing Analysis: A Review Article, in: D.A. Aaker (Hrsg.), Multivariate Analysis in Marketing: Theory \& Application, Belmont 1971, S. 314 ff.; Pohl, F., Marktsegmentierung mit multivariaten Verfahren, Diss. Münster 1977, S. 118 ff.; Green, P.E., a.a.O., S. 423 ff.; Hüttner, M., a.a.O., S. 388 .

2 Für die vorliegende Problemstellung wurde das verfügbare Clusterprogramm des Rechenzentrums der Universität Münster verwendet; vgl. Steinhausen, D., Neue Prozedur Cluster im SPSS-8 als lokale Erweiterung, in: inforum 1980, Heft 2, S. $27 \mathrm{ff}$. sowie Steinhausen, D., Langer, K., Clusteranalyse, Berlin-New York 1977, S. $118 \mathrm{ff} .$. Das implementierte Verfahren stellt relativ strenge Anforderungen an die Eingabedaten (unkorrelierte Variablen, gleiches Skalenniveau). Aus diesem Grunde erfolgte die Clusterbildung mit Hilfe der sich aus der Strukturierung des Dienstleistungsangebotes ergebenden Faktorenwerte (vgl. Kapitel C. 2. dieser Arbeit) als Inputdaten. Die Illustration der Cluster erfolgt durch eine anschliessende Rückrechnung auf den ursprünglichen Katalog der Dienstleistungsmerkmale.

$3 \mathrm{Da}$ es sich im vorliegenden Fall um einen heuristischen clusteralgorithmus handelt, der einer Gruppenvorgabe bedarf - d.h. die Zahl der cluster ist nicht selbst Ergebnis der Rechnung - wurde zur Auswahl einer geeigneten Lösung für Hersteller und Kunden jeweils eine 2er3er- und 4er-Lösung errechnet. Die endgültige Clusterzahl wird bei der Typologisierung weiter unten vorgestellt. 
3. Beschreibung der gebildeten Cluster.

a) Für jede Gruppierung sind die jeweiligen Rollenerwartungen in allen Dienstleistungsbereichen zu ermitteln. Diesen segmentspezifischen Erwartungen lassen sich die - ebenfalls segmentbezogenen - Beurteilungen der gegenwärtigen Rollenerfüllung gegenüberstellen ${ }^{1}$.

Die segmentweise Gegenüberstellung von Rollenerfüllung und -erwartung ermöglicht die Identifikation gruppenspezifischer Schwerpunkte im Dienstleistungsprogramm.

b) Eine ergänzende demographische Beschreibung der abgegrenzten Gruppen bildet die Voraussetzung für eine segmentgerechte Ansprache der Hersteller- und Kundengruppen. Hierzu eignen sich die beschriebenen allgemeinen unternehmensdemographischen, vertriebs- und einkaufsbezogenen Faktoren sowie die personenorientierten Merkmale der Kontaktpartner von Handelsvertre$\operatorname{tern}^{2}$.

Entsprechend dieser Vorgehensweise wurden die vorliegenden Rollenerwartungen der vertretenen Unternehmen und Kunden zur Segmentabgrenzung herangezogen. Die dabei gebildeten Hersteller- und Kundentypen sowie ihre Bedeutung für das Handelsvertreter-Marketing werden nachfolgend vorgestellt.

1 Vgl. Krautter, J., Zum Problem der optimalen Marktsegmentierung, in: ZfB 1975, S. $118 \mathrm{f}$..

2 Diese Merkmale - auch passive Variablen genannt - werden nicht selbst zur clusterbildung herangezogen, sondern dienen allein der demographischen Beschreibung generierter cluster; erst die gemeinsame Kenntnis von inhaltlicher Clusterstruktur (hier: Gruppen mit homogenen Rollenerwartungen) und demographischer Beschreibung der Clusterelemente ermöglicht eine Typologisierung der Marktpartner, die für die Gestaltung von Marketingmaßnahmen nutzbar ist; vgl. auch Böhler, H., a.a.O., s. 297 f.. Zur methodisch-technischen Vorgehensweise der clusterung mit passiven Variablen vgl. Steinhausen, D., a.a.O., S. 29. 


\subsection{Typologisierung der vertretenen Unternehmen}

\subsection{Festlegung der Gruppenanzahl}

Die Durchführung der Clusteranalyse zur Segmentierung des Herstellermarktes folgte einem heuristischen Vorgehen, bei dem als Alternativen 2, 3 und 4 Gruppen erzeugt wurden ${ }^{1}$. Da der Rechenalgorithmus selbst keine optimale Lösung bereitstellt ${ }^{2}$, müssen zur Festlegung der clusteranzahl sachlogische Uberlegungen herangezogen werden. Als besonders geeignet erweist sich hierfür die Anforderung an die Segmentaufteilung, hinreichend große - d.h. für Bearbeitungsmaßnahmen lohnende - Gruppierungen bereitzustellen.

Die empirischen Resultate zur 2er-Lösung belegen eine noch relativ undifferenzierte Aufteilung des Herstellermarktes. 76 \& der vertretenen Unternehmen bilden eine Gruppe ähnlicher Rollenerwartungen; 24 \& gehören der zweiten Gruppe an. Bemerkenswert ist jedoch, daß die größere Gruppe eine weitaus höhere Homogenität aufweist als das kleinere Segment ${ }^{3}$. Obwohl diese Resultate die Forderung nach hinreichender Segmentgröße weitgehend erfüllen, ist die vorgenommene Aufteilung wenig befriedigend. Beide Segmente geben Anlaß zu der Vermutung, durch eine Erhöhung der clusteranzahl differenziertere Gruppierungen abzuleiten.

1 Zur globalen Aufteilung dieser Gruppen vgl. Tabelle 9 in Anhang $I$.

2 Als mathematische Hilfsgröße läßt sich lediglich die Veränderung des Wertes der Zielfunktion (hier: Abweichungsquadratsumme aller Gruppen) heranziehen; vgl. etwa Pohl, F., a.a.O., S. 143.

3 Kennziffer für die Gruppenhomogenität ist der durchschnittliche (euklidische) Abstand der Gruppenelemente voneinander. Im vorliegenden Fall beträgt dieser 1,7 für das große und 4,4 für das kleinere Segment; vgl. Tab.9, Anhang $I$. 
Eine entsprechend durchgeführte 4er-Lösung kann den gewünschten Effekt nur teilweise erreichen. Auch bei der Bildung von vier segmenten vereint eines die Mehrzahl der vertretenen Unternehmen auf sich $\left(\begin{array}{ll}64 & 8\end{array}\right)$. Die Homogenität dieser Gruppe wächst im Vergleich zur 2er-Lösung nur geringfügig. Zwei Segmente mit 11 \& bzw. 15 \& der Hersteller weisen ein annähernd gleiches $M a B$ an Homogenität aus; das vierte Segment (10 \& der Hersteller) bleibt relativ heterogen.

Mit der Erhöhung der Segmentanzahl verbessert sich erwartungsgemäß die Homogenität der einzelnen Gruppen. Die Verbesserung ist jedoch insbesondere bei dem größten Segment nur gering. Ferner führt die Aufteilung in vier Gruppen $z u$ Segmentgrößen, die - abgesehen von dem Hauptsegment - eine Differenzierung des Instrumenteeinsatzes nur bedingt erlauben.

Betrachtet man die Resultate zur 3er-Lösung, ergibt sich die in Abbildung 57 zusammengefaßte Aufteilung der vertretenen Unternehmen.

\begin{tabular}{|c|c|c|}
\hline SEGMENT & $\begin{array}{c}\text { ANTEIL DER VERTRETENEN } \\
\text { UNTERNEHMEN } \\
\text { (IN \%) }\end{array}$ & HOMOGENI TÄTSMASS \\
\hline 1 & 11.2 & 4.7 \\
2 & 18.4 & 3.3 \\
3 & 70.4 & 1.3 \\
\hline
\end{tabular}

Abb. 57: Segmentaufteilung des Herstellermarktes 
70 \% der Hersteller bilden demnach ein relativ homogenes Segment in Bezug auf ihre Rollenerwartungen an das Dienstleistungsangebot von Handelsvertretungen. Der im Vergleich zur 4er-Lösung eingetretene Verlust an Homogenität ist nur geringfügig. Weitere 18 \& der vertretenen Unternehmen formen eine Gruppe mittlerer Homogenität; das kleinste Segment (11 \&) beinhaltet Hersteller, deren Erwartungen im Vergleich $\mathrm{zu}$ den beiden anderen Gruppen - weniger homogen ausgeprägt sind.

Insgesamt stellt die 3er-Lösung in einer zusammenfassenden Bewertung von Segmentgröße und -homogenität eine akzeptable Strukturierung des Herstellermarktes nach den Rollenerwartungen an das Dienstleistungsangebot von Handelsvertretungen dar. Inhaltliche - d.h. dienstleistungsorientierte - Segmentbeschreibung sowie demographische Segmentstruktur werden daher im folgenden für die Aufteilung des Herstellermarktes in drei Gruppen eingehender analysiert.

5.22 Inhaltliche und demographische Segmentstruktur auf Herstellerebene

Die dienstleistungsbezogene und demographische Beschreibung der gebildeten Segmente folgt der in Abbildung 57 genannten Reihenfolge. Die angegebenen Segmentbezeichnungen umschreiben schlaglichtartig den Herstellertyp der jeweiligen Gruppierung in Bezug auf das Dienstleistungsangebot von Handelsvertretungen.

\subsection{Segment I: Verkaufsorientierung mit Direktvertrieb}

Das mit 11 \& kleinste und zugleich am wenigsten homogene Segment der vertretenen Unternehmen weist die in Abbildung 58 


\section{KUNDENAETOEINUNG,}

QUAL IFT 7 IEQTE MTTARBE?TEP

AKT!JALISIEOTE KIINDENKAETE!

KENVTVTS DF: UMSATZKUNDFVST PUKTIJP

LANGFRTST? GER KUNDENKONTAKT

ERWEITERUNG DES KUIDENJK PEISET

HEDSTELLEO FRGAENZEV SIEH GUT

INF? २MAT I CNS AUST A.USCH

VERNED TUNG HERSTELLERTYF JAMATTONEN VERW LRTUNG KUNDEVTMFOTMAT? JNFN GENUEGENO INFTRMATIONEY \& V KUNDEN HEQSTELLERTNFDRMATION IIEBE? PLAVUNGEN AUSDEICHEND SERICHTE GN HFESTFLLE?

DQODUKTANRERIINGEV

BEEI NFLUSSIJNG HEDSTELLFOSIORTIMENT ANDFGUVIEN FUEP NEUE DOADIJKTF. VERTRETUNGSOPDGSAMM NACH ASTKTFORECHING

PREP SGESTALTUNG

ERMESSENSSPIELQAUM FUER KIMIDITITANCN HANDLUNGSSP! TLRAUM FUFR PPEISNACHL AESSF GESTNDEOTE VFOGIIETUNG TUSAETZL L LE:STUNG

AUSSTATTUNG DER HANDELSVETT PETUNG,

BUEP D DERSONELL BESETZT

BUE?D HAT TFCHN. HILFSM?TTEL

\section{LEISTUNGSVFPGUET!NAG}

KALKIULATION POOVI STOHSSAETZS PPJVI IIONSVกPSCHL \&G
HOC.

NIFERIG

$+$

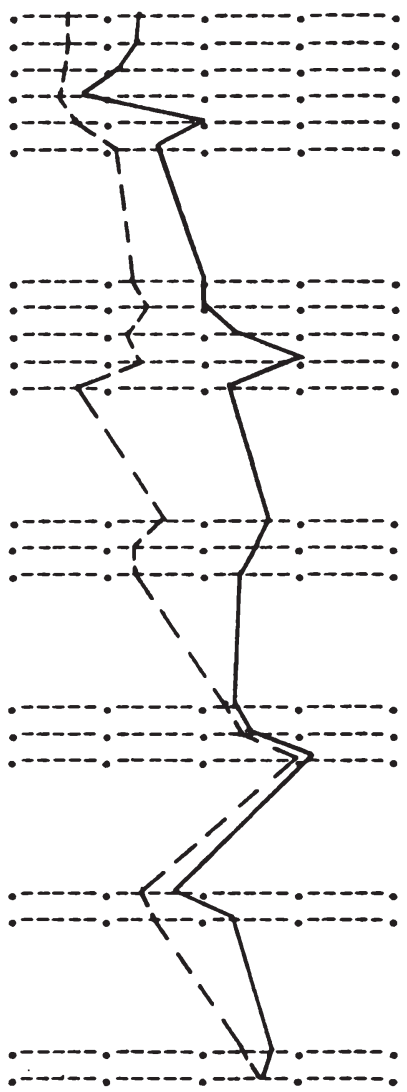

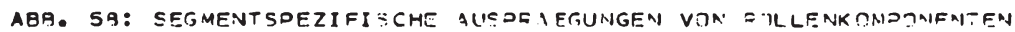
Q UF HEPSTELLEPEBFHF. -

SEGMENT I: VETKAUFSODTENTTERING MIT DPOEKTVEZT-IFO 
aufgezeigten Beurteilungen des Dienstleistungsangebotes von Handelsvertretungen auf. Die eingetragenen Profile kennzeichnen die für dieses Herstellersegment geltenden Einschätzungen der Rollenerfüllung von Handelsvertretungen sowie die segmentspezifischen Rollenerwartungen in allen Dienstleistungsbereichen von Handelsvertretungen. Im Vergleich zum Durchschnitt aller Hersteller lassen sich segmentspezifische Besonderheiten herausarbeiten ${ }^{1}$.

Gegenüber dem Durchschnitt der vertretenen Unternehmen weist das betrachtete Herstellersegment I niedrigere Beurteilungen auf. Mit Ausnahme der Konditionengestaltung wird die Rollenerfüllung von Handelsvertretungen in allen Dienstleistungsbereichen niedriger eingeschätzt. Gleichzeitig liegt das Niveau der Rollenerwartungen teilweise erheblich unterhalb des Durchschnittswertes. Die Ergänzung dieser beiden Effekte führt für segment I zu einem tendenziell geringeren Abstand der beiden Profile, d.h. der Grad der Rollenerfüllung von Handelsvertretungen wird aus Sicht der Hersteller dieses Segmentes tendenziell höher.

Dieses Resultat ist für Handelsvertretungen dennoch wenig positiv, weil die Verlagerung der Herstellerbeurteilungen den Entfaltungsspielraum für Handelsvertretungen in diesem Segment erkennbar einengt. Die beschriebene Ausnahme im Bereich der Konditionengestaltung deutet vielmehr an, das die vertretenen Unternehmen dieses Segmentes in der zusammenarbeit mit Handelsvertretungen die Prioritäten bei der Preisgestaltung sehen. Das hohe Maß an Ubereinstimmung

1 Basis dieser Profile sowie Vergleichsmaßstab zum Befragungsdurchschnitt sind die Daten in Tab. 10, Anhang $I$. 
zwischen Rollenerfüllung und -erwartung bei den erhobenen Einzelmerkmalen zur Preisgestaltung unterstreicht die Preisorientierung dieser Hersteller. Die geringen Erwartungen in den Leistungsbereichen Informationsaustausch und Ausstattung der Handelsvertretungen unterstützen gleichfalls diese Interpretation.

Die beschriebenen Abweichungen in der Beurteilung des Dienstleistungsangebotes von Handelsvertretungen sowie bei den Erwartungen dieses Herstellersegmentes legen insgesamt die Vermutung nahe, daB es sich bei diesen vertretenen Unternehmen um Hersteller handelt, die weniger am eigentlichen Dienstleistungsangebot von Handelsvertretungen interessiert sind. Ihr Interesse zielt primär auf die reine Verkaufstätigkeit von Handelsvertretern, wobei Maßnahmen der Preis- und Konditionengestaltung weitgehend der Kontrolle und Vorgabe des Herstellers unterliegen. Handelsvertretungen, die mit dem beschriebenen Herstellertyp zusammenarbeiten, werden eher als weisungsgebundene Verkaufsorgane angesehen, eine Qualifikation als selbständiges Dienstleistungsunternehmen wird dagegen von den Herstellern kaum angestrebt.

Für das Handelsvertreter-Marketing stellt sich damit die Frage, ob sich die Hersteller dieses Segmentes nach demographischen Merkmalen beschreiben lassen, die eine hinreichende Identifikation dieser vorwiegend verkaufsorientierten vertretenen Unternehmen gewährleisten. Abbildung 59 faßt für zentrale demographische Merkmale die segmentspezifischen Ausprägungen zusammen und belegt zugleich die Abweichung vom Durchschnitt der vertretenen Unternehmen ${ }^{1}$.

$1 \mathrm{Zu}$ den absoluten Ausprägungen der Durchschnittswerte vgl. Tab. 11, Anhang I. 


\begin{tabular}{|c|c|c|}
\hline HERSTELLERMERKMALE & $\begin{array}{l}\text { SEGMENT- } \\
\text { AUSPRÄGUNG }\end{array}$ & $\begin{array}{l}\text { ABWEICHUNG } \\
\text { VOM DURCH- } \\
\text { SCHNITT }(\%)\end{array}$ \\
\hline \multicolumn{3}{|l|}{ ALLGEMEINE_UNIEBNEUMENSUEBKUALE } \\
\hline ZAHL DER WARENGRUPPEN & 5,8 & +28 \\
\hline ZAHL DER BESCHÄFTIGTEN & 500 & +29 \\
\hline UMSATZ（MIO, DM） & 29.2 & -30 \\
\hline ANTEIL DES EXPORTUMSATZES（\%） & 19.3 & -1 \\
\hline ALTER DES UNTERNEHMENS（JAHRE） & 63 & +7 \\
\hline \multicolumn{3}{|l|}{ VERIBIEBSBEZQGENE_MERKMALE } \\
\hline GESAMTZAHL DER HV & 20,6 & -7 \\
\hline GESAMTZAHL DER REISENDEN & 9.5 & -4 \\
\hline UMSATZANTEIL DER HV (\%) & 54.8 & -1 \\
\hline UMSATZANTEIL DER REISENDEN（\%) & 36,1 & -2 \\
\hline $\begin{array}{l}\text { UMSATZANTEIL AUS DIREKTGESCHÄF- } \\
\text { TEN }(\%)\end{array}$ & 36,2 & +6 \\
\hline $\begin{array}{l}\text { DAUER DER ZUSAMMENARBEIT MIT } \\
\text { HV (JAHRE) }\end{array}$ & 34,8 & +11 \\
\hline \multicolumn{3}{|l|}{ PEBSONENBEZQGENE_MEBKMALE } \\
\hline ALTER & 44,0 & -2 \\
\hline UNTERNEHMENSZUGEHÖRIGKEIT（JAHRE） & 13.6 & -8 \\
\hline $\begin{array}{l}\text { QAUER PER AUSGEÜBTEN TÄTIGKEIT } \\
\text { JAHRE }\end{array}$ & 10,6 & -6 \\
\hline $\begin{array}{l}\text { ZAHL DER PERSÖNLICHEN KONTAKTE } \\
\text { MIT HV (MONATLICH) }\end{array}$ & 21,0 & -11 \\
\hline ANTEIL DER ARBEITSZEIT MIT HV (\%) & 16.0 & -17 \\
\hline
\end{tabular}

ABB, 59: DEMOGRAPHISCHE STRUKTUR VON HERSTELLERSEGMENT I 
Das als primär verkaufsorientiert beschriebene Herstellersegment I weist auch in demographischer Hinsicht eine Reihe charakteristischer Eigenarten auf, die eine relativ genaue Beschreibung dieses Herstellertyps sicherstellen. In einer Gegenüberstellung mit dem Durchschnitt aller vertretenen Unternehmen prägen insbesondere folgende Resultate dieses Herstellersegment.

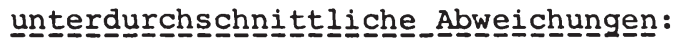

- fast ein Drittel weniger Umsatz

- geringere Vertriebsgröße, insbesondere bei den Handelsvertretern

o wesentlich geringere Kontakte mit den Handelsvertretungen

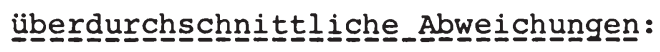

- breiteres Warenangebot

- mehr Beschäftigte

- ältere Unternehmen

- höhere Umsätze aus Direktgeschäften

- längere Zusammenarbeit mit Handelsvertretern.

Nach diesen Resultaten handelt es sich beim Herstellersegment I um eher kleinere Unternehmen hinsichtlich der Umsätze und Vertriebsstruktur, die mit überdurchschnittlich vielen Beschäftigten ein insgesamt breites Produktangebot produzieren. Die Distribution dieses umfangreichen Sortiments erfolgt seit langem mit Handelsvertretungen, wobei das Geschäft mit Großkunden vorwiegend vom Hersteller selbst abgewickelt wird. Die Intensität der zusammenarbeit mit Handelsvertretungen ist nur gering ausgeprägt.

Die demographische Kennzeichnung dieses Herstellersegmentes vermag somit die inhaltlichen tberlegungen zur Be- 
schreibung dieses Herstellertyps zu unterstützen. Die Besonderheiten demographischer und vertriebsbezogener Merkmale sowie die Befunde zur Einschätzung von Handelsvertretungen legen die Schlußfolgerung nahe, daß die vertretenen Unternehmen der beschriebenen Art vorrangig verkaufsorientiert am Markt agieren. Dabei werden zentrale Entscheidungen in der Preis- und Konditionenpolitik durch den Hersteller allein gefällt. Handelsvertretungen werden weniger aufgrund ihres umfassenden Dienstleistungsangebotes eingesetzt, sondern vielmehr als reine Verkaufsorgane angesehen.

Für Handelsvertretungen, die mit dem beschriebenen Herstellertyp zusammenarbeiten, bieten diese Resultate wertvolle Hilfen zur Gestaltung ihrer Maßnahmen. So wird insbesondere deutlich, daß eine Profilierung durch verstärkte Dienstleistungen nur wenig möglich ist. Die restriktive Handhabung der Preis- und Konditionenverantwortung steht zudem einem flexiblen Verhalten am Markt entgegen.

Die Produkte dieses Herstellertyps eignen sich demnach tendenziell eher zur Abrundung des eigenen Vertretungsprogramms. Nur im Verbund mit anderen Herstellern ist ein eigenständiges Handelsvertreter-Marketing $\mathrm{zu}$ realisieren. Bei der Akquisition von Vertretungen verkaufsorientierter Anbieter ist in jedem Falle ein tbergewicht dieses Vertretungstyps zu vermeiden, um auf Seiten der Handelsvertretung einer Adaption dieser wenig kundenorientierten Denkweise entgegenzuwirken. Der andernfalls drohende Verlust eigenständiger Aktivitäten gefährdet den Bestand der Handelsvertretung als selbständiges Dienstleistungsunternehmen. 


\subsection{Segment II: Eingeschränkte Dienstleistungsorientie- rung mit Reisendenvertrieb}

Das zweite Herstellersegment umfaßt ca. $18 \%$ der vertretenen Unternehmen. Die segmentspezifischen Ausprägungen der Beurteilung der Rollenerfüllung von Handelsvertretungen sowie der Rollenerwartungen belegen Unterschiede sowohl zum Durchschnitt aller Hersteller als auch $\mathrm{zu}$ den verkaufsorientierten Herstellern von Segment I.

Abbildung 60 stellt die spezifischen Ausprägungen der Rollenkomponenten für das Herstellersegment II gegenüber. Mit Ausnahme der Preisgestaltung werden die Leistungen von Handelsvertretungen in allen Dienstleistungsbereichen tendenziell höher eingeschätzt als im Durchschnitt der vertretenen Unternehmen. Gleichzeitig liegen die Rollenerwartungen an das Leistungsangebot von Handelsvertretungen auf einem niedrigeren Niveau. Der Grad der Rollenerfüllung ausgedrückt durch den Abstand zwischen beiden Profilen wird somit insgesamt höher angesehen. Die positivere Einschätzung durch die vertretenen Unternehmen gilt in schwächerer Form im Vergleich zum Durchschnitt aller Hersteller; in weitaus höherem Maße dagegen im Vergleich zu den verkaufsorientierten Herstellern (Segment I). Diese Resultate sprechen damit für eine in der Tendenz größere Dienstleistungsorientierung der in Segment II abgegrenzten Hersteller.

Der erkennbaren Dienstleistungsorientierung stehen die Ergebnisse zum Leistungsbereich der Preisgestaltung entgegen. Die in Segment II zusammengefaßten Hersteller beurteilen die Preis- und Konditionenverantwortung niedriger als die verkaufsorientierten Hersteller von segment $I$. Zugleich liegen die Erwartungen der Hersteller noch unterhalb der aktuellen Einschätzungen, d.h. die Verantwortung 
KUNDENGETOELIUNG

MIAL-IFIZTEDTF MITAJAFITEZ

AKT JALISIERTE KUNDENKE JTCX

KEVVTNIS DES UMEATTKUVIDFRISTOUKTUO

LANGEDISTYTE= KUUIEYKONTAKT

ENWE TTE=UNG DES KUVIDENK=EISFS

HERSTELLFE FPGAFNZEN SICH GUT

INF J = MATI DVS AUST AUSCH

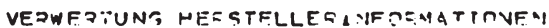
VERNERTUNG, KUSIDFNTVFOONAT IOVFN GENUEGEND IVFJPMATI ONF'I AY KUNADEN MERS TELLEOTA!FORNATTON IJERED OLAPNNNGEN AUSSFICHENT AFR:EHTE IN HESSTELLFO

\section{DDADUKTANEFGUNGFV}

HEET NFLUSSUN' HFOETELLEESMAT TME.JT ANDEGUHCEN FUER NEUE JTIDIJKTE VERT FETUVRSDEDG =AMM VARH MACKTFOESCHUJNG

DSET SGESTALTIJHG

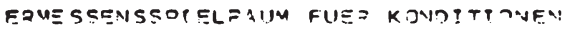
HANDLUNGSSDIELQA!JM FIJEE DEE CONDCHLAFS=E GESTNDFETE VECGUETINA, ZUSAETZL. LF?:TUNS

QUSSTATTUN. DEG HANOEL SVFETCETING

RUFZJ DFDSJNELL BECFTZT

BIEZJ HAT TECHN. HILFSN:TTEL

\section{LEISTUNGSVFSGUETUNG}

KALKULATION ORCVISTTIVSSAFTZE DODVISITVEVDOSCLLG

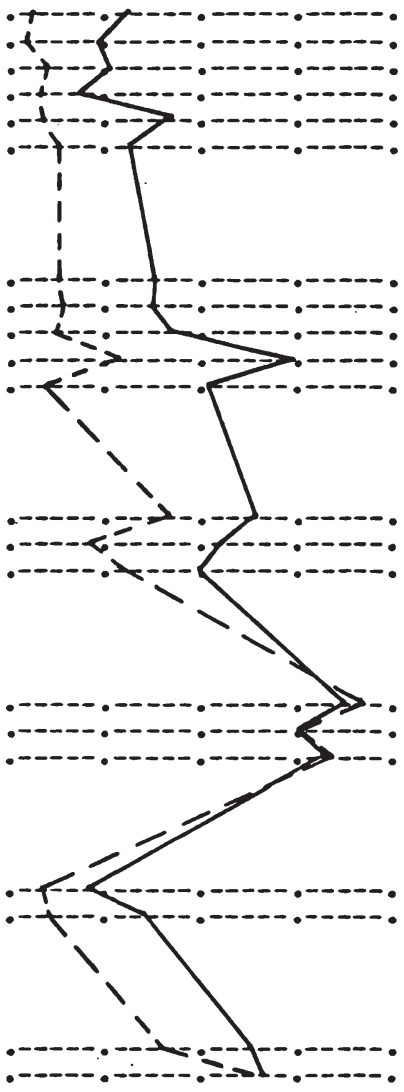

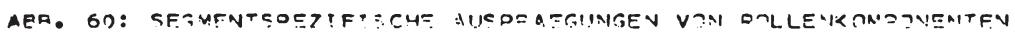
IIF HEOSTELLEOTAENE -

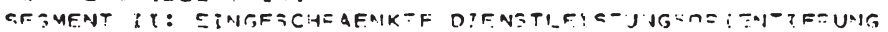

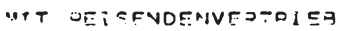

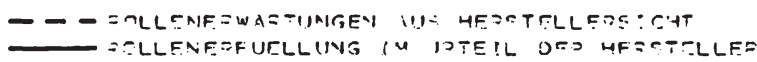


der Handelsvertretung für Preise und Konditionen sollte nicht so groß sein, wie dies gegenwärtig der Fall ist. Diese Befunde deuten einen eingeschränkten Handlungsspielraum für das Dienstleistungsangebot von Handelsvertretungen an und begünstigen mögliche Konflikte zwischen den Marktpartnern.

Ein ähnliches Konfliktpotential beinhalten die Resultate zur Vergütung zusätzlicher Leistungen. Während das gesamte Dienstleistungsangebot von Handelsvertretungen insgesamt relativ positiv eingeschätzt wird - wobei die Anforderungen der Hersteller erwartungsgemäß noch höher ausgeprägt sind -, weisen die vertretenen Unternehmen in Segment II nur eine geringe Bereitschaft zur gesonderten Abrechnung zusätzlicher Leistungen auf. Für Handelsvertretungen ist es daher von besonderem Interesse, die demographischen Merkmale der Hersteller in Segment II zu kennen, um die eigene Aktivitätenplanung segmentspezifisch ausrichten zu können und z.B. mögliche Konfliktbereiche in der Zusammenarbeit mit den vertretenen Unternehmen $z u$ antizipieren.

Abbildung 61 schlüsselt die demographischen Merkmale der dienstleistungsorientierten Hersteller von Segment II detailliert auf. Als charakteristische Merkmale sind hervorzuheben:

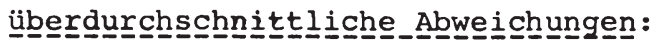

- ein Drittel höhere Umsätze

- 50 \& mehr Beschäftigte

- verstärkter Einsatz von Reisenden mit entsprechend größeren Umsatzbeiträgen

- längere Zusammenarbeit mit Handelsvertretern

- intensivere Kontakte mit Handelsvertretern

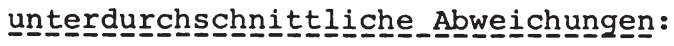

- engeres Sortimentsangebot

- geringerer Export. 


\begin{tabular}{|c|c|c|}
\hline HERSTELLERMERKMALE & $\begin{array}{l}\text { SEGMENT- } \\
\text { AUSPRÄGUNG }\end{array}$ & $\begin{array}{l}\text { ABWEICHUNG } \\
\text { VOM DURC } \\
\text { SCHNITT }(\%)\end{array}$ \\
\hline \multicolumn{3}{|l|}{ ALLGEMEINE_UNIERNEUMENSMEBKMALE } \\
\hline ZAHL DER WARENGRUPPEN & 4.2 & -9 \\
\hline ZAHL DER BESCHÄFTIGTEN & 580 & +50 \\
\hline UMSATZ（MIO.DM） & 55,4 & +34 \\
\hline ANTEIL DES EXPORTUMSATZES（\%） & 17.3 & -12 \\
\hline ALTER DES UNTERNEHMENS（JAHRE） & 60 & +2 \\
\hline \multicolumn{3}{|l|}{ YEBIRIEBSBEZOGENE_MERKMALE } \\
\hline GESAMTZAHL DER HV & 22.1 & -1 \\
\hline GESAMTZAHL DER REISENDEN & 10,4 & +5 \\
\hline UMSATZANTEIL DER HV (\%) & 55,5 & \pm 0 \\
\hline UMSATZANTEIL DER REISENDEN（\%） & 36.6 & +4 \\
\hline $\begin{array}{l}\text { UMSATZANTEIL AUS DIREKTGESCHÄF- } \\
\text { TEN }(\%)\end{array}$ & 33.5 & -2 \\
\hline $\begin{array}{l}\text { DAUER DER ZUSAMMENARBEIT MIT } \\
\text { HV (JAHRE) }\end{array}$ & 35.5 & +13 \\
\hline \multicolumn{3}{|l|}{ PER $\underline{S} \underline{N} \underline{N} \underline{N B E Z Z O G E}$ NE_MERKMALE } \\
\hline ALTER & 47.1 & +4 \\
\hline UNTERNEHMENSZUGEHÖRIGKEIT（JAHRE） & 15.4 & +4 \\
\hline $\begin{array}{l}\text { DAUER PER AUSGEÜBTEN TÄTIGKEIT } \\
(\text { JAHRE }\end{array}$ & 12.8 & +14 \\
\hline $\begin{array}{l}\text { ZAHL DER PERSÖNLICHEN KONTAKTE } \\
\text { MIT HV (MONATLICH) }\end{array}$ & 23.0 & +4 \\
\hline ANTEIL DER ARBEITSZEIT MIT HV (\%) & 19,1 & \pm 0 \\
\hline
\end{tabular}

ABB, 61: DEMOgRAPHISCHE STRUKTUR VON HERSTELLERSEGMENT II 
Das Herstellersegment II umfaßt demnach größere Unternehmen mit weit überdurchschnittlichen Umsätzen und Beschäftigtenzahlen, die jedoch im Gegensatz zu den verkaufsorientierten Herstellern (Segment I) ein erheblich engeres Sortiment anbieten. Im Vertrieb kommt den unternehmensangestellten Reisenden verstärkte Bedeutung zu; Anzahl und Umsatzbeiträge liegen im Vergleich zum Durchschnitt auf höherem Niveau. Bemerkenswert ist ferner die relativ lange Zusammenarbeit mit Handelsvertretungen, die sich zudem durch intensive Kontakte auszeichnet.

Die beschriebene Diskrepanz zwischen der grundsätzlich positiven Einschätzung des Dienstleistungsangebotes von Handelsvertretungen und einer mangelnden Bereitschaft einer differenzierten Leistungsvergütung durch die vertretenen Unternehmen in Segment II kann unter Einbeziehung der demographischen Segmentmerkmale auf die Vertriebsstruktur dieser Hersteller zurückgeführt werden. Der gleichzeitigen Einschaltung von Handelsvertretern und Reisenden wird offensichtlich nicht durch ein entsprechend differenziertes Vergütungssystem Rechnung getragen. Die empirischen Befunde sprechen vielmehr dafür, daß aus der sicht der Hersteller dieses Segmentes alle Leistungen mit der Provision abgegolten sind.

Für Handelsvertretungen, deren vertretene Unternehmen die beschriebenen Eigenschaften von Segment II aufweisen, beinhalten diese Befunde Chancen und Risiken gleichermaßen. Die positive Grundhaltung der Hersteller dieses Segmentes gegenüber dem Dienstleistungsangebot von Handelsvertretungen bietet Ansatzpunkte eines eigenständigen Handelsvertreter-Marketing. So kann beispielsweise ein umfassendes Aktivitätsprogramm zur Festigung der Beziehungen zum Hersteller beitragen oder neue Vertretungen wirksam er- 
schließen - dies war z.B. bei den primär verkaufsorientierten Herstellern von segment I nicht möglich.

Restriktiv bzw. risikobehaftet für die Maßnahmenplanung ist die Aversion der Hersteller in Segment II gegenüber einer differenzierten Leistungsvergütung. In einem Abstimmungsproze $B$ mit den vertretenen Unternehmen muß ein Handelsvertreter zwischen dem Interesse des Herstellers an seinem Dienstleistungsangebot und der Bereitschaft leistungsbezogener Vergütung genau den Weg finden, der seine Beziehungen $z u$ dem Hersteller langfristig stabilisiert und zugleich den eigenen unternehmenspolitischen Zielen gerecht wird.

Das Verhandlungsgeschick von Handelsvertretern ist hier in besonderem Maße gefordert, da die betrachteten Hersteller vielfach bereits Reisende einsetzen. Eine wenig flexible Verhandlungsführung von seiten der Handelsvertreter kann zum Verlust der Vertretung bzw. Fehlschlag bei einer Akquisition führen und somit die Ablösung durch Reisende begünstigen.

Die Argumentation gegenüber den am Dienstleistungsangebot von Handelsvertretungen interessierten Herstellern mit gemischtem Vertrieb erfordert von Handelsvertretungen eine wirksame Profilierung in den Dienstleistungsbereichen, in denen die Eigenständigkeit von Handelsvertretern gegenüber Reisenden besondere Vorteile bietet (z.B. Branchenkenntnis, Vertretungsangebot). Zur Versachlichung der Diskussion über eine leistungsspezifische Vergütung sind nachprüfbare Kalkulationsunterlagen eine wirksame Unterstützung. Erst die Verbindung eines auf die Bedürfnisse dieses Segmentes abgestimmten Dienstleistungsangebotes mit be- 
gründeten Provisions- bzw. Vergütungsforderungen vermag die Position von Handelsvertretungen bei den dienstleistungsorientierten Herstellern mit Reisendenvertrieb nachhaltig zu festigen.

\subsection{Segment III: Umfassende Dienstleistungsorientierung mit Eigenverantwortung der Handelsvertreter}

Das dritte Segment von Herstellern, das nach der Ähnlichkeit der Rollenerwartungen clusteranalytisch ermittelt wurde, umfaßt mit 70 \& den größten Anteil der vertretenen Unternehmen (vgl. Abbildung 57). Dieses Segment zeichnet sich zudem durch eine - im Vergleich zu den beiden anderen Gruppierungen - wesentlich höhere Homogenität aus. Offensichtlich stellt die Mehrzahl der Hersteller nahezu dekkungsgleiche Erwartungen an die Rolle von Handelsvertretungen im Absatzkanal. Aus der Gegenüberstellung dieser Erwartungen mit den Beurteilungen der Rollenerfüllung leiten sich daher für die Mehrzahl der Handelsvertretungen unmittelbar relevante Schlußfolgerungen für die Entwicklung des Handelsvertreter-Marketing ab.

Einen Vergleich der segmentspezifischen Ausprägungen von Rollenerfüllung und Rollenerwartung gestattet Abbildung 62 . Die von den vertretenen Unternehmen in Segment III abgegebenen Beurteilungen bewegen sich in weiten Bereichen des gesamten Dienstleistungsangebotes in Höhe der Einschätzungen des Durchschnitts aller Hersteller. Insbesondere Kundenbetreuung, Produktanregungen und Leistungsvergütung weisen kaum Abweichungen vom Durchschnitt auf. Betrachtet man in diesen Leistungsbereichen die größten Diskrepanzen zwischen Leistungsangebot (Rollenerfüllung) und erwarteter Leistung (Rollenerwartung), muß das ver- 
KUNDENAF TOEUIUNG

QUA: IF!ZTFOTE MITAORETTEE

AKTUAL? SIEDTE KUNJDENKADTEI

KEANTNTS DEE UMSATZKUNDFN'ST ZUKTUZ

LQ.NGFOIST T GER KUNDEAIK OYTAKT

ERWEITEPUNG DES KJNOFVK IE!SES

HEESTELLEP ERTAENZEN SICH GUT

\section{INFOGMATIONEAUSTAUSCH}

VERWERTUNG HEESTCLLEDINFTRMATIDHINN VEZWFRTUNG KUNDEN!NF JRNATIJNE!H GENUEGEND IVFOOMATIONEV AN KUNDEN

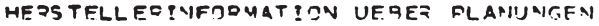
AUSAET HHENO QEQTEHTF AN HEQSTELLEO

DRODUKTANAERIUNGEN

BEEI NFLUSSUNG MEOSTFLLES SOPT IMENT ANRE GUVGEV FUEC NEUE D:RTDIJKTE VEFTRETUNGSPRJGOAMM NACH MIRKTFDP C CHUVG

PQET SGESTALTUNG

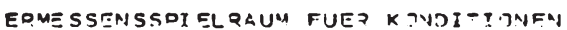
HANDLUNGSSOIELPA!JM FUFD DEE! CNACHL AESSE GESTNDSETF. VESGUETUNG ZIICAETZL. LEISTUNG

QUSSTATTUNG DFO HANTELSVEJT DETUNG

BUFOO DERSONELL AFSFT ZT

BUERD HAT TFCHN. HILF SMTTTEL

\section{LETSTINGSVERGUETINMG}

KALCULATIDN PROVICTJNESAETZE DOOVISTONSVRQSCHLGG

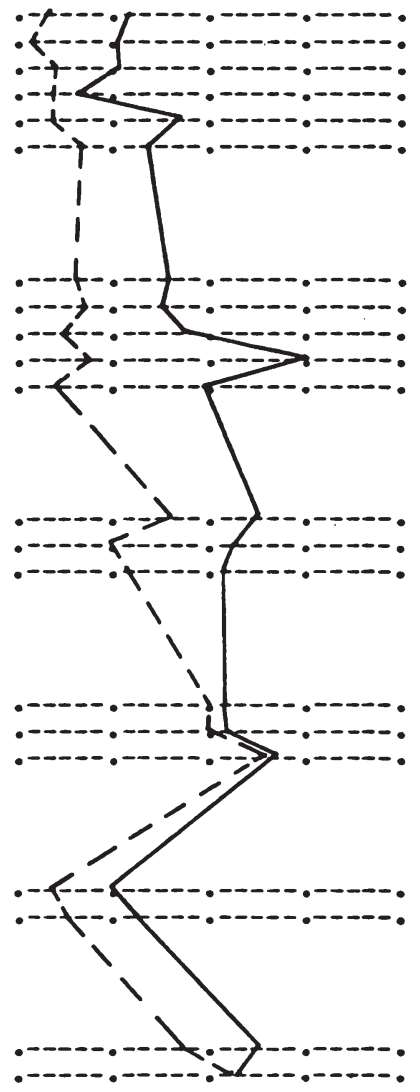

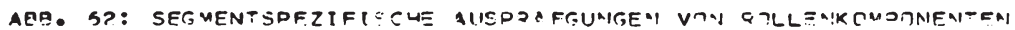
AIF HETSTFLLEDFAEVE -

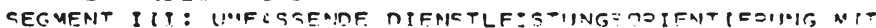
E'GENVFOANITWDOTINA DED HAVDELSVE=- JETE= 
stärkte Interesse von Handelsvertretungen der Erweiterung des Kundenkreises sowie vermehrten Anregungen für die Entwicklung neuer Produkte zukommen.

Ebenfalls keine Abweichungen vom Durchschnitt belegen die Resultate zur Einschätzung der Rollenerfüllung beim Informationsaustausch. In diesem Bereich liegen jedoch die Erwartungen hinsichtlich der kundengerichteten Informationen sowie der herstellerbezogenen Informationen über die Planungen und Zielvorstellungen der Handelsvertretungen auf einem höheren Niveau. Für die genannten Informationsaktivitäten sinkt somit der von den Herstellern dieses Segmentes wahrgenommene Grad der Rollenerfüllung. Die außerordentlich hohe Homogenität des betrachteten Herstellersegmentes gibt daher Handelsvertretungen AnlaB, speziell die genannten Informationsaktivitäten in ihrem Leistungsangebot kritisch zu überprüfen.

Deutliche Abweichungen vom Durchschnitt aller Hersteller und insbesondere von den Herstellersegmenten I und II belegen die empirischen Ergebnisse zur Preisgestaltung und Ausstattung der Handelsvertretungen beim Herstellertyp III.

Die Ausstattung der Handelsvertretungen in materieller und personeller Hinsicht wird von den betrachteten Herstellern positiver eingeschätzt. Zugleich liegen die Erwartungen an die Rolle von Handelsvertretungen in diesem Bereich höher, so daß der Grad der Rollenerfüllung keine Veränderung erfährt. Diese Betonung der innerbetrieblichen Leistungsfähigkeit deutet an, daß Handelsvertretungen für den betrachteten Herstellertyp eine bedeutsame Rolle in dessen Vertriebskonzeption zukommt.

Die Handelsvertreterorientierung der vertretenen Unternehmen in Segment III wird bei der Preisgestaltung noch deut- 
licher. Im Gegensatz zu beiden anderen Herstellertypen werden Preis- und Konditionenspielraum von Handelsvertretungen weitaus positiver angesehen. Die Erwartungen der Hersteller dieses Segmentes gehen noch darüber hinaus und unterstreichen damit die Bereitschaft der Hersteller, den Handelsvertretungen in hohem Maße eine Verantwortung für die Preis- und Konditionenpolitik einzuräumen.

Zusammenfassend dokumentieren die Befunde $\mathrm{zu}$ den segmentspezifischen Ausprägungen der Rollenkomponenten dieses Herstellertyps eine positive Grundbeurteilung von Handelsvertretungen. Die Besonderheiten bei den Rollenerwartungen der Hersteller im Segment III bieten darüber hinaus Ansatzpunkte einer ausbaufähigen Position für Handelsvertretungen und damit die Möglichkeit eines eigenständigen Handelsvertreter-Marketing. Eine demographische Identifikation des beschriebenen Herstellersegmentes ist als Voraussetzung einer entsprechenden Zielgruppenansprache unerläßlich.

Die demographische struktur von Herstellersegment III veranschaulicht Abbildung 63 im Vergleich zum Durchschnitt aller vertretenen Unternehmen. Bei globaler Betrachtung der segmentstruktur weisen die Hersteller des betrachteten Segmentes nur relativ wenige und zudem geringfügige $\mathrm{Ab}-$ weichungen vom Durchschnitt auf. Die vorliegenden Unterschiede - insbesondere im Vergleich $z u$ den beiden anderen Gruppierungen (vgl. Abbildung 59 und 61) - ermöglichen jedoch eine detailliertere Beschreibung des Herstellertyps III. Charakteristisch für diese Hersteller sind folgende Merkmale: 


\begin{tabular}{|c|c|c|}
\hline HERSTELLERMERKMALE & $\begin{array}{l}\text { SEGMENT- } \\
\text { AUSPRÄGUNG }\end{array}$ & $\begin{array}{l}\text { ABWEICHUNG } \\
\text { VOM DURCH- } \\
\text { SCHNITT }(\%)\end{array}$ \\
\hline ALLGEMEINE_UNIEBNEHMENSMERKMALE & & \\
\hline ZAHL DER WARENGRUPPEN & 4.5 & -2 \\
\hline ZAHL DER BESCHÄFTIGTEN & 318 & -18 \\
\hline UMSATZ（MIO.DM） & 39.7 & -4 \\
\hline ANTEIL DES EXPORTUMSATZES（\%） & 20.2 & +3 \\
\hline ALTER DES UNTERNEHMENS（JAHRE） & 57 & -4 \\
\hline \multicolumn{3}{|l|}{ VERIRIEBSBEZQQGENE_UEBKMALE } \\
\hline GESAMTZAHL DER HV & 22.6 & +1 \\
\hline GESAMTZAHL DER REISENDEN & 9.8 & -1 \\
\hline UMSATZANTEIL DER HV（\%） & 55.7 & \pm \\
\hline UMSATZANTEIL DER REISENDEN（\%) & 34.8 & -1 \\
\hline $\begin{array}{l}\text { UMSATZANTEIL AUS DIREKTGESCHÄF- } \\
\text { TEN }(\%)\end{array}$ & 34.0 & \pm 0 \\
\hline $\begin{array}{l}\text { DAUER DER ZUSAMMENARBEIT MIT } \\
\text { HV (JAHRE) }\end{array}$ & 29,8 & -5 \\
\hline \multicolumn{3}{|l|}{ PEBSONENBEZOGENE_MEBKMALE } \\
\hline ALTER & 44.8 & -1 \\
\hline UNTERNEHMENSZUGEHÖRIGKEIT（JAHRE） & 14.8 & \pm 0 \\
\hline $\begin{array}{l}\text { DAUER PER AUSGEÜBTEN TÄTIGKEIT } \\
\text { (JAHRE) }\end{array}$ & 10,9 & -3 \\
\hline $\begin{array}{l}\text { ZAHL DER PERSÖNLICHEN KONTAKTE } \\
\text { MIT HV (MONATLICH) }\end{array}$ & 24.5 & +3 \\
\hline ANTEIL DER ARBEITSZEIT MIT HV (\%) & 19,5 & +2 \\
\hline
\end{tabular}

ABB， 63: DEMOGRAPHISCHE STRUKTUR VON HERSTELLERSEGMENT III 
- geringere Beschäftigtenzahl

- tendenziell niedrigerer Umsatz

- jüngere Unternehmen

- kürzere Zusammenarbeit mit Handelsvertretern

- tendenziell mehr Handelsvertreter (Anzahl und Umsatzbeiträge)

- intensivere persönliche Kontakte mit den Handelsvertretern.

Die beschriebenen Besonderheiten deuten insgesamt darauf hin, daß es sich bei den Herstellern dieses Typs um eher kleinere Unternehmen handelt, die im Vertrieb vorwiegend Handelsvertreter einsetzen. Die kürzere Zeit der Zusammenarbeit mit Handelsvertretern erklärt sich aus dem niedrigeren Alter der vertretenen Unternehmen. Die Beziehungen $z u$ den Handelsvertretern zeichnen sich durch überdurchschnittliche Kontakthäufigkeiten aus; dies zeigt besonders ein Vergleich mit den verkaufsorientierten Herstellern aus Segment I.

In Verbindung mit den inhaltlichen Resultaten zur Einschätzung von Rollenerfüllung und Rollenerwartung des Herstellertyps III läßt sich das beschriebene Segment zusammenfassend als klassische Domäne der Handelsvertretertätigkeit bezeichnen. Hersteller mit den beschriebenen demographischen Merkmalen (vgl. Abbildung 63) stellen demnach die Kernzielgruppe eines Handelsvertreter-Marketing dar.

Der Maßnahmenplanung und Gestaltung stehen dabei von seiten der vertretenen Unternehmen dieses Typs weitaus weniger Hemmisse entgegen als in den beiden anderen Segmenten, wo eine einseitige Verkaufsorientierung (Segment I) oder Restriktionen bei bestimmten Leistungen (Segment II) der Entfaltung eines eigenständigen Handelsvertreter-Marketing 
entgegenstehen. Hersteller des betrachteten Segmentes weisen vielmehr eine umfassende Dienstleistungsorientierung mit weitgehender Eigenverantwortlichkeit der Handelsvertretungen auf.

Eine Orientierung des Handelsvertreter-Marketing an den homogenen Erwartungen dieses Herstellertyps stellt somit ein hersteller- bzw. zielgruppengerechtes Leistungsangebot sicher und bewahrt zugleich einen Freiraum für die Durchsetzung handelsvertreterspezifischer Zielvorstellungen. Gelingt den Handelsvertretungen eine Profilierung ihres Marketing gegenüber den vertretenen Unternehmen, können sie andererseits auf die Wünsche und Bedürfnisse der Abnehmer in höherem Maße eingehen.

Zur Einbindung der kundengerichteten Aktivitäten in das herstellerorientierte Marketing von Handelsvertretungen ist die Frage zu prüfen, ob sich auf der Ebene der Abnehmer in ähnlicher Weise Segmente abgrenzen lassen, die sich durch homogene Erwartungen an das Leistungsangebot von Handelsvertretungen auszeichnen.

\subsection{Typologisierung der Abnehmer}

Die Abgrenzung von Segmenten auf Kundenebene, die in ihren Erwartungen an die Rolle von Handelsvertretungen eine homogene Struktur aufweisen, erfolgte analog zu den vertretenen Unternehmen auf clusteranalytischer Basis. Dementsprechend ist vor der inhaltlichen und demographischen Beschreibung der gebildeten Gruppierungen eine für die vorliegende Problemstellung adäquate Anzahl von Segmenten $z u$ bestimmen ${ }^{1}$.

1 Die methodische Vorgehensweise sowie die inhaltiche Umsetzung orientierten sich an der in Capitel C. 5.1 beschriebenen struktur. 


\subsection{Festlegung der Gruppenanzah 1}

Für den Kundenmarkt wurden zur Ermittlung einer geeigneten Clusterzahl ebenfalls eine 2er-,3er- und 4er-Gruppierung erzeugt ${ }^{1}$. Aus diesen Alternativen ist entsprechend der Anforderungen an die Kriterien zur Segmentierung des Kundenmarktes die für das Handelsvertreter-Marketing geeignete Gruppenanzahl zu bestimmen.

Die auf der Grundlage der Homogenität von Erwartungen an die Rolle der Handelsvertretungen im Absatzkanal vorgenommene Aufteilung des Kundenmarktes in zwei Gruppen führt zu einer ähnlich unbefriedigenden segmentstruktur wie auf der Herstellerebene. Bei der 2er-Lösung umfaßt ein Segment 83 \& aller Abnehmer; das zweite die verbleibenden $17 \%$. Als bemerkenswert ist herauszustellen, daß auch beim Kundenmarkt das größere Segment ein höheres Maß an Homogenität aufweist als das kleinere. Im Vergleich $z u$ den vertretenen Unternehmen sind ferner beide Kundensegmente jeweils homogener.

Eine Verdoppelung der Gruppenzahl weist für die Segmentstruktur Effekte auf, die bereits auf Herstellerebene zu beobachten waren. Bei der 4er-Lösung vereint das größte Segment mit der zugleich höchsten Homogenität 53 \& der Abnehmer auf sich. Zwei weitere Gruppen mittlerer Homogenität umfassen 18 \& bzw. $21 \%$ der Abnehmer. Eine vierte Gruppe von Kunden $(8 \%)$ bleibt vergleichsweise heterogen.

Ein Vergleich der 4er-Lösung mit der 2er-Lösung deckt auf, daß primär das größte Kundensegment $\left(\begin{array}{ll}83 & \text { \& }\end{array}\right)$ eine weitere Unterteilung erfahren hat, wobei ein Teil dieser umfassenden Gruppe als eigenes Segment herausgefiltert wird

1 Zur umfassenden Strukturierung dieser Gruppen vgl. Tab. 12, Anhang I. 
(Segment 3 mit 21 \& bei der 4er-Lösung). Damit führt die Aufteilung des Kundenmarktes in vier Segmente zu einem Ergebnis, das den Anforderungen an eine für Bearbeitungsmaßnahmen lohnende Segmentgröße zunächst genügt.

Der Versuch einer inhaltlichen Beschreibung der gebildeten vier Gruppen spricht jedoch nicht für eine Beibehaltung dieser Marktaufteilung. Als hauptsächliches Hemmnis erweist sich die beschriebene Abspaltung des Segmentes mit 15 \& der Kunden. Eine Gegenüberstellung der inhaltlichen und demographischen Segmentmerkmale mit dem Segment größter Homogenität (53 \&) ließ vielmehr keine eindeutige Abgrenzung dieser beiden Gruppierungen zu. Die Unterschiede in der Beurteilung der gegenwärtigen Rollenerfüllung sowie bei den Rollenerwartungen waren uneinheitlich; auch eine demographische Fixierung der Segmentschwerpunkte war nicht möglich. Aus diesem Grunde wird auch der Kundenmarkt von Handelsvertretungen in drei Segmente untergliedert. Abbildung 64 veranschaulicht die Marktaufteilung der 3er-Lösung.

\begin{tabular}{|c|c|c|}
\hline SEGMENT & $\begin{array}{c}\text { ANTEIL DER ABNEHMER } \\
\text { (IN \%) }\end{array}$ & HOMOGENITÄTSMASS \\
\hline 1 & 67,3 & 0,8 \\
2 & 23,7 & 1,9 \\
3 & 9,0 & 2,9 \\
\hline
\end{tabular}

Aob. 64: Segmentaufteilung des Kundenmarktes 
Zwei Drittel der Abnehmer von Handelsvertretungen zeichnen sich nach den vorliegenden empirischen Befunden durch ein außerordentlich hohes $M a B$ an Homogenität in Bezug auf die Erwartungen an die Rolle von Handelsvertretungen im Absatzkanal aus. Eine Gruppe mittlerer Homogenität umfaßt 24 \& der Abnehmer. Die verbleibenden 9 \& der Kunden sind im Vergleich zu den beiden anderen Gruppen in ihren Erwartungen an das Dienstleistungsangebot von Handelsvertretungen weniger homogen.

Die Maßnahmenplanung im Handelsvertreter-Marketing benötigt zur Entwicklung segmentspezifischer Aktivitäten detaillierte Informationen über die inhaltlichen Unterschiede der Segmente, d.h. über die möglicherweise divergierenden Erwartungen. Zur gezielten Ansprache der Segmente ist darüber hinaus eine Kenntnis der demographischen Segmentstrukturen erforderlich.

Die Analyse einzelner Rollenkomponenten auf Kundenebene hat bei den betrachteten demographischen Merkmalen teilweise einen EinfluB der Betriebsform - und in geringerem MaBe auch der Branche - herausgestellt. Zur Uberprüfung der grundlegenden Frage, ob sich die Kundensegmente allein auf Unterschiede der Betriebsformen begründen (z.B. Industriebetriebe versus Handelsbetriebe), wurde die entsprechende Verteilung der in die Untersuchung einbezogenen Betriebsformen und Branchen auf die segmente ermittelt ${ }^{1}$.

Die Resultate erbrachten jedoch weder für die Betriebsformen noch für die Wirtschaftsbereiche signifikante Unterschiede zwischen den drei gebildeten Segmenten. Demnach

1 Zur Aufteilung der einbezogenen Betriebsformen und Branchen vgl. Tab. 8 , Anhang I. 
hängen homogene Erwartungen an das Leistungsangebot von Handelsvertretungen von seiten der Abnehmer weniger von dem generellen Unternehmenstyp ab. Aufgabe der nachfolgenden Segmenttypologisierung muß es daher sein, die spezifischen segmentprägenden Unterschiede herauszuarbeiten, die eine hinreichende Ansprache der identifizierten $\mathrm{Ab}-$ nehmersegmente gewährleisten.

\subsection{Inhaltliche und demographische Segmentstruktur auf Kundenebene}

\subsection{Segment I: Dienstleistungsorientierte Abnehmer mittlerer Größe}

Das größte Segment von Kunden, das sich durch eine weitgehend homogene Erwartung an das Leistungsangebot von Handelsvertretungen auszeichnet, umfaßt mit 67 \& die Mehrzahl der Abnehmer von Handelsvertretungen. Bereits dieses Resultat hat für das Handelsvertreter-Marketing eine unmittelbare Bedeutung.

Die außerordentlich hohe Homogenität dieser Kundengruppe und die Größe dieses Segmentes implizieren für die strategische Planung von Maßnahmen gegenüber dem Kundenmarkt ein relativ einheitliches Vorgehen. Ein auf die Bedürfnisse des vorliegenden Kundensegmentes abgestelltes Dienstleistungsangebot bildet den Kern der Handelsvertretertätigkeit, der für die beiden anderen noch $z u$ diskutierenden Gruppierungen einer segmentspezifischen Anpassung bedarf.

Dieses Segment, dem das Hauptinteresse von Handelsvertretungen gelten muß, zeichnet sich durch die in Abbildung 65 aufgezeigten Beurteilungen der gegenwärtigen Rollenerfül- 
HOCH

NIEDRLG

DOE I SGF STAL TUNG

DPMFS SFV SOIELOAIM FIIES KJA:O:T TENEN

HANDLUNGTSDISLDGUM FUEO DNEISNSEHLAESSE

CE TDUKT ANDE GUNCEN

DVDEGUNGEV FUE= MIEUE DPINUKTE

7FE?:IFL.UK: SUNG YEニSTFLLESSOETTMENT

VERTEETUNGSPPDGRMMM NACH MAEKTFOLSCHUNG

VERWF PTIJMG KUNDEVI IFR = WA T T TNEV

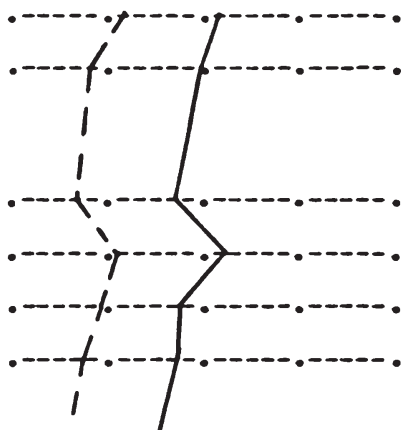

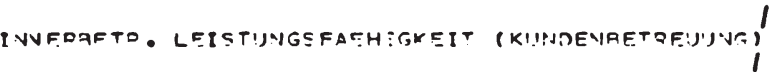

SUEOJ HAT YECHN. HYLFGMITTTL

PUEJO OFOSTNELL AESETZT

DUALIFIZTEOTE NIT Q.PAEITSO

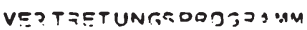

HEPTTELLER EPGAENZEN EIT.H GUT

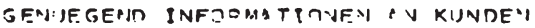

LANGF PT ET thEO KUNDENKJNITKT

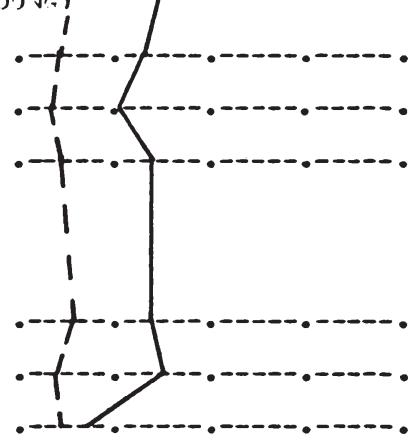

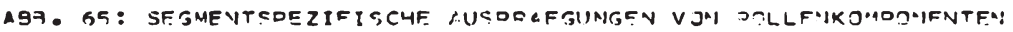

AUF KUNDENESENE -

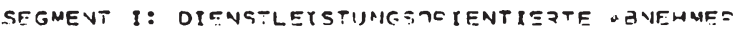

NITTLECFP GDSFSSE

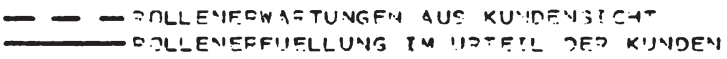


lung sowie Rollenerwartungen an Handelsvertretungen aus. Charakteristika dieses Kundentyps arbeitet ein Vergleich zum Durchschnitt aller befragten Abnehmer heraus ${ }^{1}$.

Die Kundengruppe vom Typ I beurteilt die Rollenerfüllung von Handelsvertretungen in allen Dienstleistungsbereichen höher als die Abnehmer im Durchschnitt. Gleichzeitig liegen die Erwartungen an die Rolle der Handelsvertretungen auf einem höheren Niveau. Beide Resultate bringen in ihrem Zusammenwirken eine positive Grundhaltung dieses Kundentyps gegenüber Handelsvertretungen zum Ausdruck.

Betrachtet man die Schwerpunkte bei den einzelnen Leistungsbereichen, müssen Handelsvertretungen insbesondere der innerbetrieblichen Leistungsfähigkeit Aufmerksamkeit widmen, weil hier die Erwartungen dieser Kundengruppe am größten sind. Eine hinreichende innerbetriebliche Leistungsfähigkeit besitzt dabei allein wenig akquisitorisches Potential ${ }^{2}$, sondern ist als Voraussetzung des gesamten organisatorischen Ablaufs unabdingbar ${ }^{3}$.

Im Bereich der Produktanregungen sinkt der Grad der Rollenerfüllung von Handelsvertretungen vor allem hinsichtlich der Einflußnahme auf das Herstellersortiment sowie der Verwertung von Kundeninformationen. Mit Priorität bei der Verarbeitung der von den Kunden erhaltenen Informationen sind die genannten Schwachpunkte abzubauen. Da auch die Resultate auf Herstellerebene eine ähnliche Tendenz aufzeigten, ist insgesamt anzuregen, beiden Marktpartnern die Funktion von Handelsvertretungen als neutralem In-

1 Basis der Profile in Abbildung 65 sowie Vergleichsmaßstab zum Befragungsdurchschnitt sind die Daten in Tab. 13, Anhang $I$.

$2 \mathrm{Vgl}$. Gutenberg, E., a.a.0., S. $237 \mathrm{f} .$.

3 Vgl. Meffert, H., Kimmeskamp, G., a.a.O., S. 24. 
formationsverarbeiter und Meinungsbildner in weitaus stärkerem Maße als bisher $\mathrm{zu}$ verdeutlichen. Die empirischen Befunde belegen, daß auf Hersteller- und Kundenebene insbesondere bei dem betrachteten Abnehmersegment - ein hoher Bedarf für eine Informationsumsetzung im Rahmen der Produkt- und Sortimentsentwicklung entsteht.

Eine weitere Unterscheidung des Kundentyps I vom Durchschnitt betrifft die Preisgestaltung. Der angesprochene Effekt einer positiveren Einschätzung der Rollenerfüllung und höheren Rollenerwartungen gilt auch für diesen Leistungsbereich. Die Verlagerung der empirischen Resultate führt jedoch für das betrachtete Kundensegment zu einer Vergrößerung des Abstandes zwischen beiden Profilen, d.h. den Grad der Rollenerfüllung von Handelsvertretungen beurteilen die Abnehmer dieses Segmentes weniger positiv.

Gemessen an den Erwartungen dieser umfangreichen Kundengruppe und den gegenläufigen Interessen der drei Herstellertypen belegen diese Resultate ein Dilemma für die Planung von Maßnahmen im Handelsvertreter-Marketing. Während alle Gruppierungen von vertretenen Unternehmen einer Ausweitung der preis- und konditionenpolitischen Verantwortung von Handelsvertretungen eher abweisend gegenüberstehen, erwartet der größte Teil der Abnehmer eine höhere Flexibilität von den Handelsvertretungen.

Eine Beilegung dieser konträren Einschätzungen erfordert von den Handelsvertretern eine BewuBtmachung dieser Problematik bei den vertretenen Unternehmen. Dabei kann das Interesse von Handelsvertretungen weniger auf eine Erhöhung ihrer Kompetenz generell abzielen, vielmehr ist den Herstellern eine verantwortungsbewußte Handhabung preis- und konditionenpolitischer Ermessensspielräume nachzuweisen. 
Mit Blick auf die Abnehmer des beschriebenen Segmentes stärkt ein derartiges Vorgehen die Position von Handelsvertretung und Hersteller am Markt gleichermaßen.

Die Ausrichtung der Maßnahmenplanung bzw. die gezielte Ansprache der im vorliegenden segment zusammengefaßten Kunden erfordert eine geeignete demographische Identifikation dieser Abnehmergruppe. Da sich Branche bzw. Betriebsform als nicht trennscharfe Kriterien zwischen den gebildeten Kundentypen erwiesen haben ${ }^{1}$, wurden zur Abgrenzung der Kundentypen die bekannten einkaufsbezogenen Faktoren, allgemeine Unternehmensmerkmale sowie personale Variablen der Kontaktpartner von Handelsvertretern bei ihren Abnehmern herangezogen ${ }^{2}$. Abbildung 66 stellt die Ausprägungen dieser Merkmale für den Kundentyp I vor und belegt die Abweichungen vom Durchschnitt aller Abnehmer.

Bei globaler Betrachtung der Ausprägungen der demographischen Variablen des Kundentyps I belegen die empirischen Befunde bei einer Reihe von Merkmalen nur geringe Abweichungen vom Durchschnitt. Dennoch lassen sich - unter Einbeziehung der Resultate für die beiden anderen noch zu diskutierenden Segmente - einige Besonderheiten dieses Kundentyps erkennen, die eine detailliertere Kennzeichnung und damit zielgruppengerechte Ansprache erlauben ${ }^{3}$. Als segmentprägende Merkmale sind hervorzuheben:

- durchschnittliche Größenordnungen allgemeiner Unternehmensmerkmale

1 Vgl. Kapitel C. 5.31 dieser Arbeit.

2 Vgl. hierzu im Detail die Ubersicht in Abbildung 8 .

$3 \mathrm{Vgl}$. Tabelle 14, Anhang I. 


\begin{tabular}{|c|c|c|}
\hline MERKMALE DER KUNDEN & $\begin{array}{l}\text { SEGMENT- } \\
\text { AUSPRÄGUNG }\end{array}$ & $\begin{array}{l}\text { ABWE ICHUNG } \\
\text { VOM DURCH- } \\
\text { SCHNITT }(\%)\end{array}$ \\
\hline \multicolumn{3}{|l|}{ ALLGEMEINE_UNIERNEUMENSMEBKMALE } \\
\hline ZAHL DER WARENGRUPPEN & 16.0 & \\
\hline ZAHL DER BESCHÄFTIGTEN & 1.729 & +5 \\
\hline UMSATZ（MIO.DM） & 143.0 & +3 \\
\hline ALTER DES UNTERNEHMENS（JAHRE） & 57 & \pm 0 \\
\hline \multicolumn{3}{|l|}{ EINKAUESBEZQGENE__MEBKUALE } \\
\hline ZAHL DER EINKÄUFER & 8.5 & \pm 0 \\
\hline $\begin{array}{l}\text { AUFTRAGSABWICKLUNG MIT TELEFON/ } \\
\text { TELEX (\%) }\end{array}$ & 32.8 & + \\
\hline EINKAUFSANTEIL VON REISENDEN (\%) & 19.3 & + \\
\hline EINKAUFSANTEIL VON VERTRETERN (\%) & 23.5 & +1 \\
\hline $\begin{array}{l}\text { EINKAUFSANTEIL VON HERSTELLERN } \\
\text { DIREKT }(\%)\end{array}$ & 53.2 & -3 \\
\hline EINKAUFSANTEIL ÜBER ZENTRALE（\%） & 37.5 & -2 \\
\hline \multicolumn{3}{|l|}{ PEBSONENBEZOGENE_MEBKMALE } \\
\hline ALTER & 45.6 & -1 \\
\hline $\begin{array}{l}\text { DAUER PER UNTERNEHMENSZUGEHÖRIGKEIT } \\
(\text { JAHRE })\end{array}$ & 18.1 & +2 \\
\hline $\begin{array}{l}\text { DAUER DER AUSGEÜBTEN TÄTIGKEIT } \\
(\text { JAHRE) }\end{array}$ & 12.5 & \pm \\
\hline $\begin{array}{l}\text { ZAHL DER PERSÖNLICHEN KONTAKTE } \\
\text { MONATLICH) }\end{array}$ & 44.3 & +2 \\
\hline $\begin{array}{l}\text { ANTEIL DER ARBEITSZEIT MIT HANDELS- } \\
\text { VERTRETERN }(\%)\end{array}$ & 11,1 & \pm 0 \\
\hline
\end{tabular}

ABB, 66: DEMOGRAPHISCHE STRUKTUR VON KUNDENSEGMENT I 
- relativ höhere Einkaufsanteile von Reisenden und Handelsvertretern ${ }^{1}$

- relativ niedrigere Einkaufsanteile beim Direktbezug bzw. einer Einkaufszentrale ${ }^{1}$.

Die Abnehmer des betrachteten Kundensegmentes lassen sich demnach als Unternehmen mittlerer Größe kennzeichnen (1.800 Beschäftigte, 143 Mio. DM Umsatz), deren Einkauf durch ca. acht Einkäufer abgewickelt wird. Die Auftragsabwicklung mit den Handelsvertretungen erfolgt zu knapp einem Drittel nicht durch persönliche Besuche; dieser Wert liegt über dem Durchschnitt aller Abnehmer.

Hinsichtlich der Bezugsquellen des Kundentyps I ist eine leichte Bevorzugung des persönlichen Verkaufs zu erkennen. Die Einkaufsanteile von Handelsvertretern - und auch von Reisenden - liegen sowohl im Vergleich zum Durchschnitt als auch zu den beiden anderen Segmenten auf einem höheren Niveau. Der Direktbezug von Herstellern sowie der Bezug über eine Einkaufszentrale dominieren zwar den gesamten Einkauf wertmäßig, das beschriebene Kundensegment weist aber gerade bei diesen Bezugsquellen unterdurchschnittliche Werte auf. Für das tendenziell größere Gewicht des persönlichen Verkaufs spricht ferner die höhere $\mathrm{Zahl}$ der persönlichen Kontakte, die die Einkäufer monatlich mit den Handelsvertretern haben.

1 Diese beiden Entscheidungen betreffen weniger die absolute Höhe der jeweiligen Einkaufsanteile, als vielmehr die Verschiebung in Relation $\mathrm{zu}$ den beiden anderen Segmenten, vgl. Tabelle 14, Anhang I. 
Inhaltliche und demographische Kennzeichnung des Kundentyps I lassen zusammenfassend Unternehmen mittlerer Größenordnungen mit einer Verschiebung bei den Bezugsquellen zugunsten persönlicher Kontakte als Hauptzielgruppe eines abnehmerorientierten Handelsvertreter-Marketing hervortreten, wobei eine tendenziell positive Grundhaltung gegenüber Handelsvertretungen deutlich wurde. Als charakteristisches Merkmal dieses Kundentyps ist die außerordentlich hohe Homogenität der Erwartungen an die Rolle von Handelsvertretungen $\mathrm{zu}$ betonen.

Für das Handelsvertreter-Marketing belegen diese Resultate die Chance einer weitgehend einheitlichen Gestaltung des gesamten Dienstleistungsangebotes gemä $\beta$ den Anforderungen der beschriebenen Hauptzielgruppe. Eine Profilierung gegenüber dieser Hauptzielgruppe - und damit auch gegenüber den Reisenden als unmittelbaren Konkurrenten - kann insofern relativ einheitlich erfolgen.

Die duale Einbindung von Handelsvertretungen in den $\mathrm{Ab}-$ satzkanal erfordert darüber hinaus eine Abstimmung mit den als konfliktär herausgearbeiteten Dienstleistungsbereichen, die auf seiten der Hersteller von gegenläufigem Interesse sind. Eine positive Umsetzung dieser Konfliktbereiche festigt die Marktstellung einer Handelsvertretung und fördert zugleich ihre Eigenständigkeit als Absatzmittler.

Die demographische Identifikation der Hauptzielgruppe eines abnehmerorientierten Handelsvertreter-Marketing konnte fur diese dienstleistungsorientierten Kunden mittlerer Größe nur relativ global erfolgen, weil sich kaum gravierende Abweichungen vom Durchschnitt der Abnehmer herausgestellt haben. Die Ansprache der Hauptzielgruppe wird jedoch erleichtert, zieht man - quasi als Negativabgrenzung - die inhaltlichen und insbesondere die demogra- 
phischen Besonderheiten der beiden anderen Kundensegmente ergänzend heran.

\subsection{Segment II: Kleinkunden mit geringer Handelsver- treterorientierung}

Das zweite auf der Kundenebene abgegrenzte segment umfaßt 24 \& der Abnehmer von Handelsvertretungen. Verglichen mit der Hauptzielgruppe zeichnet sich dieses Segment durch ein geringeres $M a B$ an Homogenität aus, das dennoch eine segmentorientierte Anpassung des Dienstleistungsangebotes rechtfertigt.

Abbildung 67 veranschaulicht die segmentspezifischen Ausprägungen der Rollenerfüllung und Rollenerwartung aus der Sicht der Abnehmer. Im Vergleich zum Durchschnitt der befragten Kunden und insbesondere zur Hauptzielgruppe zeichnet sich das vorliegende Kundensegment durch eine niedrigere Einschätzung beider Rollenkomponenten aus. Das gegenwärtige Leistungsangebot von Handelsvertretungen wird weniger positiv eingeschätzt; gleichzeitig liegen die Erwartungen auf einem niedrigeren Niveau. Trotz dieser Verlagerung in den Beurteilungen ändert sich der Grad der Rollenerfüllung - d.h. der Abstand zwischen den beiden Profilen - nur unwesentlich.

Konsequenz dieses Resultates für das Handelsvertreter-Marketing gegenüber dieser Kundengruppe ist ein insgesamt niedrigeres Aktivitätsniveau in allen Leistungsbereichen. Gleichzeitig ist zu fragen, warum die Abnehmer dieses Segmentes die aktuellen Leistungen der Handelsvertreter niedriger einschätzen als die Hauptzielgruppe. Obwohl die vorliegende Studie diese Frage nicht unmittelbar beantworten kann, läßt sich vermuten, daß beispielsweise eine 
DRF. I SGE STAL TUNS

ERAESSEVSjOIELAAUM FUE? KOAITTIOAEN

HANOLUNGS SPIELRA!JM FUF. DPFISNACHL AESSF.

PRJDUKT ANDE GUNGEN

A NRECIJVGFY FUEP NE!JE PITDUKTE

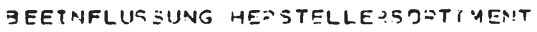

VFRTRETUNGSPODSSAMM NGCH MAFKTFOESCHUNG

VEDINEQTUNIG KU IDENS NFTOM.TIONFN

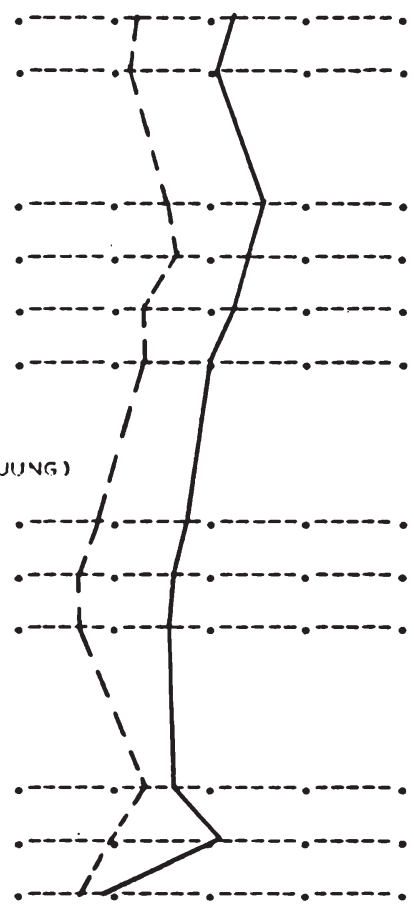

- ANGFQISTIGEO KUNOENKONTAKT

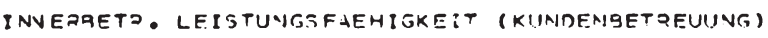

BUEPO HAT TESHV. HILFSMTTTFL

BUEPO DFOSONFLL PESFTZT

QUALIFI ZIEGTE M!T + PGEITE?

VEDT RFTUNGCDDOSDAMM

HERSTELLER EPGAENZEN כTCH GUT

GENUEGEND INFDZMAT?ONEN IN KUNDFN 
schwächere Interaktion zwischen Handelsvertretung und Abnehmer dieses Segmentes zu der niedrigen Beurteilung geführt hat. Sollte diese Annahme bei der demographischen Segmentbeschreibung (z.B. bei der Kontakthäufigkeit zwischen Handelsvertreter und Einkäufer) eine Unterstützung erhalten, leitet sich für die Maßnahmenplanung daraus das Erfordernis $a b$, dem hier beschriebenen Kundentyp das Leistungsangebot von Handelsvertretungen transparenter zu machen. Schwerpunkt der Aktivitäten gegenüber dieser Kundengruppe ist damit weniger eine Leistungsausweitung, sondern die Bewußtmachung der bisherigen Leistungsfähigkeit bei Kunden des betrachteten Typs II.

Ansatzpunkte der genannten Art sind bei der innerbetrieblichen Leistungsfähigkeit erkennbar. So werden insbesondere die technische Ausstattung der Handelsvertretung sowie die ständige Erreichbarkeit im Vergleich zum Durchschnitt und zur Hauptzielgruppe schlechter eingeschätzt. Möglicherweise fehlen den Kunden lediglich detaillierte Informationen über die eingesetzten Maßnahmen zur Kundenbetreuung.

In ähnlicher Weise sind den Abnehmern dieses Segmentes wegen der weit unterdurchschnittlichen Beurteilung die Aktivitäten der Handelsvertretung bei der Einflußnahme auf die Produktentwicklung der vertretenen Unternehmen transparent zu machen. Für diese Notwendigkeit spricht zugleich das von den betrachteten Kunden wahrgenommene Informationsdefizit aus der zusammenarbeit mit den Handelsvertretungen.

Als wesentliches Resultat der Beurteilungen dieses Kundentyps bleibt festzuhalten, daB die Abnehmer des betrachteten 
Segmentes eine wesentlich geringere Affinität zum Dienstleistungsangebot von Handelsvertretungen aufweisen. Eine ergänzende demographische Beschreibung dieses Kundentyps unterstützt diese Interpretation und stellt zugleich die notwendigen Informationen zur Identifikation dieser Abnehmergruppe bereit.

Aus der in Abbildung 68 dargestellten Gruppenstruktur sind vor allem nachfolgende Merkmale als dominant segmentprägend hervorzuheben.

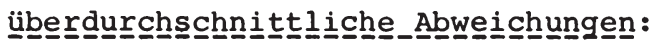

- mehr Warengruppen

- höherer Einkaufsanteil über eine Zentrale

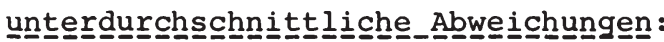

o weniger Beschäftigte

- erheblich weniger Umsatz

- weniger Einkäufer

- geringere Einkaufsanteile von Handelsvertretern

- niedrigere Kontaktintensität mit Handelsvertretern

Der Kundentyp II umfaßt nach diesen Resultaten in erster Linie kleinere Unternehmen, deren Zusammenarbeit mit Handelsvertretungen weniger stark ausgeprägt ist als bei der beschriebenen Hauptzielgruppe. Dies zeigt sich vor allem bei den Bezugsquellen sowie dem Ausmaß der persönlichen Kontakte zwischen Einkäufern und Handelsvertretern. Während der wertmäßige Einkaufsanteil von Handelsvertretungen erheblich unterdurchschnittliche Größenordnungen aufweist, liegt der Anteil des Einkaufs, der über eine Einkaufszentrale abgewickelt wird, auf einem überdurchschnittlichen Niveau. 


\begin{tabular}{|c|c|c|}
\hline MERKMALE DER KUNDEN & $\begin{array}{l}\text { SEGMENT- } \\
\text { AUSPRÃGUNG }\end{array}$ & $\begin{array}{l}\text { ABWEICHUNG } \\
\text { VOM DURCH } \\
\text { SCHNITT }(\%)\end{array}$ \\
\hline \multicolumn{3}{|l|}{ ALLGEMEINE_UNIEBNEUMENSMEBKMALE } \\
\hline ZAHL DER WARENGRUPPEN & 23,2 & +33 \\
\hline ZAHL DER BESCHÄFTIGTEN & 1.222 & -26 \\
\hline UMSATZ（MIO.DM） & 84.5 & -40 \\
\hline ALTER DES UNTERNEHMENS（JAHRE） & 55 & -3 \\
\hline \multicolumn{3}{|l|}{ EINKAUESBEZOGENE_MEBKMALE } \\
\hline ZAHL DER EINKÄUFER & 6.4 & -25 \\
\hline $\begin{array}{l}\text { AUFTRAGSABWICKLUNG MIT TELEFON/ } \\
\text { TELEX }(\%)\end{array}$ & 30,3 & -5 \\
\hline EINKAUFSANTEIL VON REISENDEN（\%） & 17,9 & -5 \\
\hline EINKAUFSANTEIL VON VERTRETERN（\%） & 19,6 & -16 \\
\hline $\begin{array}{l}\text { EINKAUFSANTEIL VON HERSTELLERN } \\
\text { DIREKT }(\%)\end{array}$ & 55,9 & +2 \\
\hline EINKAUFSANTEIL ÜBER ZENTRALE（\%) & 40,8 & +7 \\
\hline \multicolumn{3}{|l|}{ PEBSONENBEZOGENE_MEBKMALE } \\
\hline ALTER & 42,4 & -6 \\
\hline $\begin{array}{l}\text { DAUER DER UNTERNEHMENSZUGEHÖRIG- } \\
\text { KEIT (JAHRE) }\end{array}$ & 15,4 & -12 \\
\hline $\begin{array}{l}\text { DAUER PER AUSGEÜBTEN TÄTIGKEIT } \\
\text { JAHRE }\end{array}$ & 11,4 & - \\
\hline $\begin{array}{l}\text { ZAHL DER P.ERSÖNLICHEN KONTAKTE } \\
\text { MONATLICH) }\end{array}$ & 43.1 & \pm 0 \\
\hline $\begin{array}{l}\text { ANTEIL DER ARBEITSZEIT MIT } \\
\text { HANDELSVERTRETERN }(\%)\end{array}$ & 10,5 & -5 \\
\hline
\end{tabular}

ABB, 68: DEMOGRAPHISCHE STRUKTUR VON KUNDENSEGMENT II 
Die Verschiebungen in der Bezugsquellenstruktur bestärken damit die Aussage, daß es sich bei dem beschriebenen Kundentyp um eher kleinere Unternehmen handelt. Die höhere Bedeutung von Einkaufszentralen läßt z.B. auf Einzelhandelsunternehmungen schließen, die Mitglieder von Einkaufsgenossenschaften sind. Die niedrigere Kontaktintensität mit den Handelsvertretungen dokumentiert sich vorwiegend in dem geringeren Anteil der Arbeitszeit der Einkäufer, der auf die Zusammenarbeit mit den Handelsvertretern entfällt, während die absolute Anzahl der persönlichen Kontakte nur leicht rückläufig ist.

Das beschriebene Kundensegment vom Typ II zeichnet sich nach den Resultaten der empirischen Untersuchung zusammenfassend durch erheblich niedrigere Erwartungen an die Rolle von Handelsvertretungen aus. Die demographische Aufschlüsselung des Segmentes führte zu dem Ergebnis, daß diese Abnehmergruppe vorwiegend kleinere Unternehmen beinhaltet.

Die Kennzeichnung dieser Kundengruppe impliziert für das Handelsvertreter-Marketing eine relativ problemlose Segmentbearbeitung. Ausgehend von dem zu realisierenden höheren Aktivitätsniveau in allen Leistungsbereichen bei der Hauptzielgruppe, ist es ohne größere Anpassungsmaßnahmen möglich, den Schwachpunkten im Leistungsangebot wirksam zu begegnen, die von den kleineren Abnehmern besonders negativ vermerkt wurden (z.B. im Bereich der hersteller- und kundengerichteten Informationen).

Eine alleinige Konzentrierung auf diesen Abnehmertyp ist nach den vorliegenden Resultaten nur bedingt angezeigt. Die Stellung der Handelsvertretung als selbständiger $\mathrm{Ab}-$ satzmittler und damit die Entwicklung eines eigenständigen Dienstleistungsangebotes werden durch dieses Kundensegment weder gefordert noch entscheidend begünstigt. 
Der quantitative Umfang dieser Kundengruppe spricht grundsätzlich für eine Bearbeitung dieses Segmentes. Als zusätzliche Entscheidungskriterien müssen jedoch die Umsatzbeiträge dieser Kundengruppe sowie mögliche Herstellerforderungen nach umfassender Marktabdeckung ergänzend berücksichtigt werden.

\subsection{Segment III: Preisorientierte Großabnehmer}

Das dritte auf der Kundenebene nach Maßgabe der Rollenerwartungen abgegrenzte segment umfaßt mit 9 \% der Abnehmer die kleinste Gruppe. Im Vergleich $\mathrm{zu}$ beiden anderen Segmenten ist diese zugleich am wenigsten homogen.

Die in Abbildung 69 dokumentierte inhaltliche Segmentstruktur bringt einige gravierende Unterschiede zum Durchschnitt aller Kunden und $\mathrm{zu}$ den beiden anderen Gruppierungen zum Ausdruck; insbesondere im Leistungsbereich der Preisgestaltung erweisen sich die Befunde als atypisch.

Die Kundengruppe vom Typ III beurteilt die Aktivitäten von Handelsvertretungen hinsichtlich ihrer Ermessensspielräume für die Preis- und Konditionengestaltung weitaus negativer als die beiden anderen Gruppierungen. Umso erstaunlicher sind die Resultate für die Erwartungen an die Rolle von Handelsvertretungen in diesem Leistungsbereich. Abnehmer dieses Segmentes verneinen nahezu vollständig eine anzustrebende Verantwortung von Handelsvertretungen bei der Preis- und Konditionenfestlegung.

Denkbar ist, daß die Aversion dieser Kundengruppe sich gegen einen langwierigen Proze $B$ der Preisverhandlungen richtet. Im Vorgriff auf die demographische Segmentbeschreibung erfährt diese Annahme eine Bestätigung dadurch, 


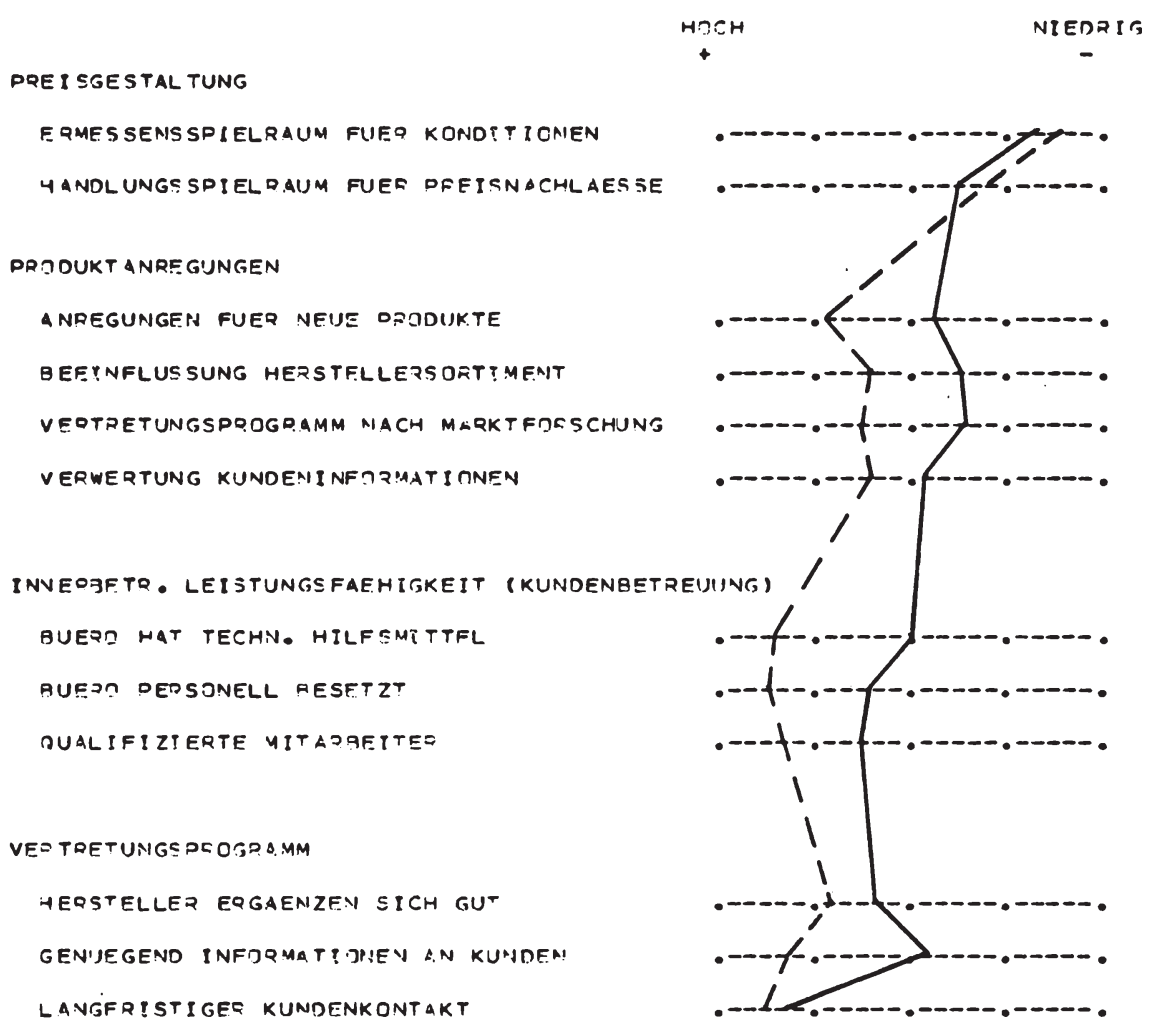

AB9. 69: SEGMEगTSPEZIFISCHE AUSPOAEGUNGEN VJV UILLEVKOMOQNENTFN DUF KUNDENEGFNF -

SEGMENT III: DQEISORIENTIERTE GOOSSA QNEHMF?

- - DOLLFNERW ARTUNGEN aUS KIJNOENSYCht

- ODLLEAEFFUFLLUNG IM UPTF'L DEZ KUNDEN 
daß es sich bei der betrachteten Kundengruppe um Großabnehmer handelt. Ihr Einkaufsvolumen und damit letztlich eine gewisse Nachfragemacht erlauben es diesem Abnehmerkreis, den Verhandlungsproze $B$ über Preise und Konditionen $z u$ verkürzen und unmittelbar das äußerste Angebot zu verlangen.

Die übrigen Leistungsbereiche von Handelsvertretungen weisen sämtlich unterdurchschnittliche Einschätzungen auf. Insbesondere die Beurteilung der gegenwärtigen Rollenerfüllung fällt wenig positiv aus; die Resultate liegen teilweise noch niedriger als bei den Abnehmern aus Segment II. In ähnlicher Weise bewegen sich die Erwartungen an das Dienstleistungsangebot von Handelsvertretungen auf einem niedrigeren Niveau.

Diese Verschiebungen führen bei einigen Leistungsmerkmalen zu einer erheblichen Vergrößerung des Abstandes zwischen den Profilen, d.h. der Grad der Rollenerfüllung von Handelsvertretungen wird niedriger eingeschätzt. Dies gilt vor allem für eine marktgerechte zusammensetzung des Vertretungsprogramms von Handelsvertretungen sowie für die kundengerichteten Informationen und die innerbetriebliche Leistungsfähigkeit in technischer Hinsicht. Den genannten Bereichen muB daher bei einer Anpassung des Leistungsniveaus das primäre Interesse von Handelsvertretern zukommen.

Die inhaltliche Beschreibung dieses Kundentyps läßt sich nach diesen Resultaten als mittlere Dienstleistungsorientierung mit vollständiger Ablehnung einer Preis- und Konditionenverantwortung von Handelsvertretungen zusammenfassen. Den demographischen Merkmalen der beschriebenen 
Kundengruppe kommt demnach eine besondere Bedeutung $z u$, da eine möglichst exakte Identifikation dieser Abnehmer potentielle Konflikte im Bereich der Preisgestaltung vermeiden kann.

Die demographischen Ausprägungen der Abnehmer vom Typ III faßt Abbildung 7o zusammen. Als segmentprägende Besonderheiten sind nachfolgende Merkmale hervorzuheben:

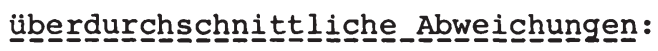
- ein Drittel mehr Beschäftigte
- 80 \& höhere Umsätze
- zwei Drittel mehr Einkaufspersonal
- ein Drittel höhere Einkaufsanteile von Handelsvertretern
- höhere Einkaufsanteile beim Direktbezug von Herstellern
- mehr Zeitaufwand für die Kontakte mit Handelsvertretern

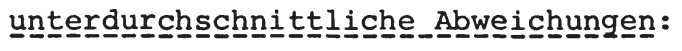

- weniger Warengruppen
- geringere Einkaufsanteile von Reisenden
- weniger Kontakte (absolut) mit Handelsvertretern.

Die demographische Struktur des beschriebenen Kundentyps weist vorwiegend Merkmale eines typischen Großabnehmers auf. Bereits die ailgemeinen unterdemographischen Faktoren stellen diese Interpretation eindrucksvoll unter Beweis. Bemerkenswert ist auch bei diesem Kundensegment, daß eine Differenzierung nach Betriebsformen nicht anzutreffen ist. Die als 'Großabnehmer' bezeichnete Kundengruppe umfaßt demnach sowohl Abnehmer aus dem industriellen Bereich (z.B. in der Investitionsgüterbranche) als auch Kunden auf Handelsebene (z.B. Einkaufsverbände, Warenhäuser). 


\begin{tabular}{|c|c|c|}
\hline MERKMALE DER KUNDEN & $\begin{array}{l}\text { SEGMENT- } \\
\text { AUSPRÄGUNG }\end{array}$ & $\begin{array}{l}\text { ABWEICHUNG } \\
\text { VOM DURCH } \\
\text { SCHNITT }(\overline{\%})\end{array}$ \\
\hline \multicolumn{3}{|l|}{ ALLGEMEINE_UNIEBNEUMENSMEBKMALE } \\
\hline ZAHL DER WARENGRUPPEN & 12,2 & -29 \\
\hline ZAHL DER BESCHÄFTIGTEN & 2.183 & +32 \\
\hline UMSATZ（MIO.DM） & 251,9 & +81 \\
\hline ALTER DES UNTERNEHMENS（JAHRE） & 55 & -3 \\
\hline \multicolumn{3}{|l|}{ EINKAUESBEZZOGENE_MERKMALE } \\
\hline ZAHL DER EINKÄUFER & 14.2 & +67 \\
\hline $\begin{array}{l}\text { AUFTRAGSABWICKLUNG MIT TELEFON/ } \\
\text { TELEX }(\%)\end{array}$ & 29,8 & +7 \\
\hline EINKAUFSANTEIL VON REISENDEN（\%) & 16,9 & -10 \\
\hline EINKAUFSANTEIL VON VERTRETERN（\%） & 31,4 & +35 \\
\hline $\begin{array}{l}\text { EINKAUFSANTEIL VON HERSTELLERN } \\
\text { DIREKT }(\%)\end{array}$ & 61,7 & +13 \\
\hline EINKAUFSANTEIL ÜBER ZENTRALE（\%) & 37,6 & -2 \\
\hline \multicolumn{3}{|l|}{ PERSONENBEZOGENE_MERKMALE } \\
\hline ALTER & 48,6 & +8 \\
\hline $\begin{array}{l}\text { DAUER DER UNTERNEHMENSZUGEHÖRIG- } \\
\text { KEIT (JAHRE) }\end{array}$ & 20,3 & +15 \\
\hline $\begin{array}{l}\text { DAUER PER AUSGEÜBTEN TÄTIGKEIT } \\
(J A H R E)\end{array}$ & 16,5 & +31 \\
\hline $\begin{array}{l}\text { ZAHL DER PERSÖNLICHEN KONTAKTE } \\
\text { MONATLICH) }\end{array}$ & 37,6 & -13 \\
\hline $\begin{array}{l}\text { ANTEIL DER ARBEITSZEIT MIT HANDELS- } \\
\text { VERTRETERN }(\%)\end{array}$ & 12,1 & +10 \\
\hline
\end{tabular}

ABB, 70: DEMOGRAPHISCHE STRUKTUR VON KUNDENSEGMENT III 
Die Bezugsquellenstruktur dieser Abnehmer verzeichnet eine sehr große Verschiebung zugunsten von Handelsvertretungen. Gleichzeitig sinkt der Einkaufsanteil von Reisenden, während der Herstellerdirektbezug ansteigt. Für das Handelsvertreter-Marketing sind diese Resultate unmittelbar relevant, da eine derartige Chance zur Erzielung hoher Auftragsvolumen bei keinem der beiden anderen Segmente gegeben war.

Als Problembereich der Bearbeitung dieser Großabnehmer erweist sich deren Kontaktverhalten gegenüber Handelsvertretern. Der Anteil der Arbeitszeit der Einkäufer, der auf die zusammenarbeit mit den Handelsvertretern entfällt, ist überdurchschnittlich hoch. Auf der anderen seite haben die Einkäufer bei absoluter Betrachtung erheblich weniger Kontakte mit Handelsvertretern. Die durchschnittliche Kontaktdauer (bei einem Besuch oder Telefongespräch) ist damit in dieser Kundengruppe besonders hoch.

Dieser Befund beinhaltet positive Aspekte fủr das einzelne Gespräch, bei dem z.B. umfassende Angebote vorgelegt werden können. Problematisch ist dagegen die geringere absolute Zahl der Kontakte zwischen Einkäufern und Handelsvertretungen. Offensichtlich wird es für Handelsvertreter zunehmend schwierig, einen Gesprächstermin bei den Einkäufern der GroBabnehmer zu erhalten. Die verminderte Kontaktchance belegt damit zugleich die Notwendigkeit eines Leistungsangebotes, das den spezifischen Bedürfnissen dieses Kundentyps entspricht.

Ein zielgruppengerechtes Handelsvertreter-Marketing dient dabei nicht allein der Profilierung gegenüber den Reisenden anderer Hersteller. Ein kundengerechtes Leistungsangebot fördert - angesichts der hohen Einkaufsanteile über Handelsvertreter - darüber hinaus eine wirksame Abgrenzung von anderen Handelsvertretungen. 
Für die Zusammenarbeit mit den vertretenen Unternehmen beinhaltet die inhaltliche und demographische Kennzeichnung der Großabnehmer vor allem die Konsequenz, im Bereich der Preisgestaltung die Kompetenzen klar abzugrenzen. In jedem Falle sind nach den vorliegenden Einschätzungen der Kunden kurzfristige Entscheidungen - auch mit direktem Kontakt zwischen vertretenem Unternehmen und Abnehmer langwierigen Verhandlungsprozessen (z.B. mit zeitaufwendigen Rückfragen) vorzuziehen.

\subsection{Zusammenfassende Hypothesenbeurteilung der rollenbe- zogenen Segmentierung}

Ausgangspunkt einer Segmentierung der Marktpartner von Handelsvertretungen war die Uberlegung, durch eine Abgrenzung homogener Subgruppen auf beiden Marktebenen Ansatzpunkte für ein differenziertes Handelsvertreter-Marketing aufzudecken. Als Segmentierungskriterium wurden dazu die an die Rolle von Handelsvertretungen im Absatzkanal gestellten Erwartungen der vertretenen Unternehmen und Kunden herangezogen.

Die Gruppenbildung erfolgte methodisch durch das Verfahren der Clusteranalyse. Unter Abwägung inhaltlicher Aspekte hat sich sowohl für den Hersteller- als auch für den Kundenmarkt eine Aufteilung in jeweils drei Segmente als sinnvoll erwiesen.

Die empirischen Resultate folgen mit diesen Gruppierungen den theoriegeleiteten Erwartungen. Die Hypothese der Segmentabgrenzung kann nach den vorliegenden Befunden für Hersteller- und Kundenmarkt gleichermaßen akzeptiert werden. 
Auf beiden Marktebenen ist es gelungen, homogene Subgruppen zu identifizieren, die sich jeweils durch unterschiedliche Erwartungen an die Rolle von Handelsvertretungen auszeichnen. Die Beschreibung der einzelnen Segmente konnte zur Präzisierung der Konsequenzen für das Handelsvertreter-Marketing durch eimesegmentspezifische Gegenüberstellung der Beurteilungen des aktuellen Leistungsangebotes (Rollenerfüllung) mit den Erwartungen in den einzelnen Dienstleistungsbereichen (Rollenerwartung) wirksam ergänzt werden.

Die Möglichkeit der inhaltichen Segmentabgrenzung gewährleistet allein keine hinreichende Basis zur gezielten Ansprache der Gruppierungen. Aus diesem Grunde verfolgte die Hypothese der Segmentbeschreibung die Annahme, daß sich die gebildeten Gruppierungen durch unternehmens- und personenbezogene Merkmale voneinander unterscheiden.

Die empirische Untersuchung bestätigt grundsätzlich auch diese Hypothese. Die entsprechende Identifikation der Segmente konnte sich dabei nicht allein auf die absoluten Ausprägungen demographischer Merkmale in den einzelnen Segmenten stützen. Schwerpunkte einzelner Herstellerund Kundentypen zeigten sich dagegen deutlich in den relativen Veränderungen einzelner Merkmale im Vergleich zum Durchschnitt der Befragten sowie insbesondere in einem Vergleich der Ausprägungen demographischer Faktoren der Segmente untereinander.

Als Fazit der rollenbezogenen Segmentierung ist herauszustellen, daß sich Handelsvertretungen auf beiden Marktseiten jeweils einer Hauptzielgruppe gegenübersehen. Die Erwartungen an das Dienstleistungsangebot von Handelsver- 
tretungen bzw. ihre Rolle im Absatzkanal deuten dabei insgesamt die Möglichkeit einer relativ einheitlichen Profilierung gegenüber beiden Marktpartnern an. Aktuelle Schwachstellen belegen alle Segmente im Bereich der Informationsaktivitäten. Potentielle Konfliktfelder in der zusammenarbeit mit den Herstellern bzw. Kunden sind die Preis- und Konditionenverantwortung sowie die Leistungsvergütung.

Ein an den grundlegenden Erwartungen der Marktpartner orientiertes Dienstleistungsangebot bildet nach den Befunden der empirischen Studie den Kern des HandelsvertreterMarketing gegenüber den Hauptzielgruppen auf beiden Marktseiten. Periphere Anpassungen an die Bedürfnisse der übrigen Segmente können darüber hinaus die Position von Handelsvertretungen im vertikalen Marketing stärken und damit letztlich eine langfristige Absicherung ihrer Eigenständigkeit als Dienstleistungsunternehmen sicherstellen. 


\section{Implikationen der Untersuchung zum Marketing von Han- delsvertretungen}

Ausgehend von einem wenig befriedigendem Stand der theoretischen und empirischen Forschung zum HandelsvertreterMarketing wurde in der vorliegenden Arbeit versucht, unter Heranziehung eines verhaltenswissenschaftlichen Bezugsrahmens die Stellung von Handelsvertretungen im $\mathrm{Ab}$ satzkanal marketingorientiert aufzuarbeiten. Die Untersuchungsergebnisse sind daher sowohl für die weitere theoriegestützte Forschung als auch für das Marketing von Handelsvertretungen unmittelbar relevant.

\section{Auswirkungen für die weitere Forschung}

Das theoretische Gerüst der vorliegenden Arbeit stellten die in jüngerer zeit in der distributionspolitischen Forschung diskutierten Ansätze der Rollentheorie bereit. Auf der Basis allgemeiner rollentheoretischer Ubberlegungen sowie vorliegender Ansätze im Bereich des vertikalen Marketing wurde ein handelsvertreterspezifisches Rollenmodell entwickelt, das die duale Einbindung der Handelsvertretung in den Absatzkanal wiederzugeben vermochte. Dieses Modell bildete die Grundlage zur Ableitung und Konkretisierung einer Reihe theoriegeleiteter Hypothesen, die einer empirischen Uberprüfung unterzogen werden konnten.

In Beantwortung der eingangs formulierten Programmfragen läßt sich die Eignung des rollentheoretischen Untersuchungsansatzes beurteilen.

(1) Die Operationalisierung der Rollenkonstrukte durch die Beurteilung des Dienstleistungsangebotes von Handelsvertretungen hat sich grundsätzlich als geeignet 
erwiesen, zentrale Rollen von Handelsvertretungen abzugrenzen.

(2) Im Hinblick auf die Gestaltung von Maßnahmen im Rahmen eines Handelsvertreter-Marketing konnte ferner eine Reihe von Bestimmungsfaktoren herausgearbeitet werden, die sich sowohl für die Rollenkomponenten einzelner Marktpartner als auch für die Rollenbeziehungen als erklärungswirksam erwiesen haben.

(3) Die Kenntnis dieser Einflußfaktoren versetzte in die Lage, konkrete Maßnahmenempfehlungen in einzelnen Dienstleistungsbereichen $\mathrm{zu}$ formulieren.

(4) Die Heterogenität der rollenbezogenen Beurteilung des Marketing von Handelsvertretungen machte eine rollenorientierte Segmentierung des Hersteller- und Kundenmarktes erforderlich. Mittels dieser psychographischen Marktstrukturierung ließen sich zentrale Zielgruppen eines Handelsvertreter-Marketing auf beiden Marktseiten identifizieren und sowohl inhaltlich - d.h. dienstleistungsbezogen - als auch demographisch voneinander abgrenzen.

In einer zusammenfassenden Beurteilung des Untersuchungsansatzes hat sich damit das Rollenkonzept in seiner operationalisierten Form für das Handelsvertreter-Marketing als tragfähiger Forschungsansatz erwiesen. Die empirischen Resultate belegen zugleich Anregungen weiterführender Forschungen in methodischer Hinsicht sowie im Hinblick auf das Handelsvertreter-Marketing.

Einen ersten Ansatzpunkt bieten die Resultate zur Strukturierung des Dienstleistungsangebotes. Die auf der Basis 
der Rollenausuibung von Handelsvertretungen sowie der Rollenerfüllung und -erwartung aus der Sicht der Marktpartner vorgenommene Systematisierung ließ das gesamte Leistungsangebot von Handelsvertretungen als heterogenes Maßnahmenbündel erscheinen ${ }^{1}$. Die hohe Eigenständigkeit einer Reihe von Dienstleistungen regt für weitere Untersuchungen die Verwendung einer reduzierten Anzahl von Einzelmerkmalen an. Eine gezielte Auswahl zentraler Leistungsmerkmale oder eine Beschränkung auf einen Leistungsbereich - der dabei weiter $z u$ differenzieren wäre - verspricht detaillierte Aussagen, die auch in methodischer Hinsicht höheren Anforderungen genügen.

Einen zweiten Ansatzpunkt zukünftiger Forschung belegt die Analyse der Bestimmungsfaktoren auf allen diskutierten Modellebenen. Die multiplen Zusammenhänge zwischen den aggregierten Dienstleistungsbereichen und den unternehmens- und personendemographischen Merkmalen der befragten Handelsvertretungen, Hersteller und Kunden konnten sich teilweise nur auf relativ geringe Erklärungsbeiträge stützen.

Für die im Rahmen der vorliegenden Untersuchung angestrebten Erkenntnisse über grundlegende Beeinflussungsrichtungen konnten diese Befunde die benötigten Tendenzaussagen bereitstellen. Zur Absicherung dieser Resultate ist in weiterführenden Studien ein besonderer Schwerpunkt auf die

1 Die Heterogenität zeigt sich beispielsweise in den erklärten Varianzbeiträgen im Rahmen der Faktorenanalyse. Durchschnittlich 40 \& erklärte Varianz deuten auf die Existenz hoher Einzelrestfaktoren d.h. auf eine weitgehende Unabhängigkeit der zugrundeliegenden Dienstleistungsmerkmale; vgl. Kapitel C. 2. dieser Arbeit. 
Heranziehung weiterer Einflußgrößen $z u$ legen, die für das Handelsvertreter-Marketing relevant sind. Hier sind beispielhaft psychographische Kriterien oder Kennziffern über die Erfahrung und Zufriedenheit mit Handelsvertretern $\mathrm{zu}$ nennen.

Als grundlegendes methodisches Problem ist schließlich das unterschiedliche Datenniveau der erforderlichen Bestimmungsfaktoren $z u$ nennen (z.B. Umsätze vs. Kriterien der Einkaufsorganisation). Bisherige Methoden sind nur wenig befriedigend in der Lage, Skalierungsunterschiede wirksam auszugleichen bzw. geeignete Interpretationshilfen bereitzustellen.

Theoretischer und empirischer Anlage der vorliegenden Untersuchung kommt zusammenfassend der Charakter einer Basisstudie $z u$, die den grundlegenden Rahmen eines Handelsvertreter-Marketing aufspannte. Die Beantwortung differenzierterer Fragestellungen in Dienstleistungsbereichen muß weiteren darauf aufbauenden Detailstudien vorbehalten bleiben.

Entsprechend dieser Einordnung lassen sich einige grundlegende Implikationen der Untersuchungsergebnisse für die strategische Orientierung eines Handelsvertreter-Marketing aufzeigen.

2. Auswirkungen für das strategische HandelsvertreterMarketing

Die Rollenanalyse des Handelsvertreter-Marketing konnte für das Dienstleistungsangebot von Handelsvertretungen in allen Teilbereichen wertvolle Anhaltspunkte für die konkrete Gestaltung einzelner Aktivitäten bereitstellen. 
Insbesondere den Erwartungen der Marktpartner kam aufgrund ihres projektiven Charakters eine besondere Bedeutung zu.

Für eine strategische Ausrichtung des HandelsvertreterMarketing sind in diesem Zusammenhang die Befunde der rollenbezogenen (erwartungsorientierten) Segmentierung der Marktpartner herauszustellen. Ausgehend von der dualen Einbindung einer Handelsvertretung zwischen Herstellerund Kundenmarkt ergeben sich unmittelbar die zentralen strategischen Stoßrichtungen eines HandelsvertreterMarketing ${ }^{1}$.

Abbildung 71 führt die dualen Marketingkonzeptionen im Rahmen des Handelsvertreter-Marketing mit den Zielgruppen auf beiden Marktseiten zusammen. Die Abbildung stellt damit zugleich die Notwendigkeit eines dualistischen Handelsvertreter-Marketing anschaulich unter Beweis.

Die abnehmergerichtete Marketingkonzeption muß sich primär auf die Hauptzielgruppe des Kundenmarktes konzentrieren. Die Beurteilungen und insbesondere Erwartungen dieser Kundengruppe bilden den Ausgangspunkt der kundengerichteten Maßnahmenplanung.

Die herstellergerichtete Marketingkonzeption hat in erster Linie die Bedürfnisse des auf seiten der vertretenen Unternehmen abgegrenzten Hauptsegmentes zu berücksichtigen.

1 Vgl. Meffert, H., Marketing, a.a.0., s. $223 \mathrm{ff.;}$ derselbe, Die Handelsvertretung im Spannungsfeld des Marketing-Systems, a.a.0., S. $138 \mathrm{f}$.. 


\section{SEGMENT I:}

VERKAUT SURIENTIERUNG MIT DIREKTVERTRIEB

\section{SEGMENT 11:}

EINGESCHRÄNKTE DIENSTLEISTUNGSORIENTIERUNG MIT REISENDENVERTRIEB

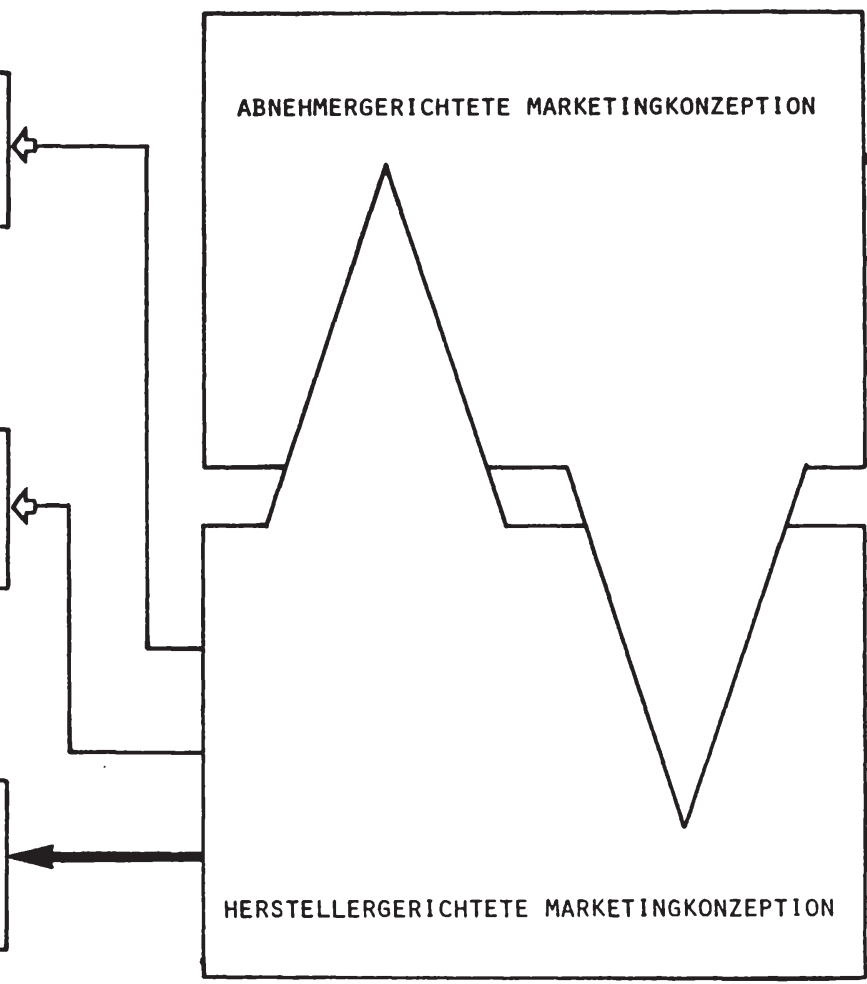

SEGMENT II:

KLE INKUNDEN MIT GERINGER HANDELSVERTRETERORIENTIERUNG 
Die Konzeptionen gegenüber vertretenen Unternehmen und Kunden sind dabei nicht als separate Komponenten $\mathrm{zu}$ verstehen. Aufgabe der Handelsvertreter ist es vielmehr, beide Konzeptionen so aufeinander abzustimmen, daß Synergieeffekte bestmöglich genutzt werden. Dieses integrierte Handelsvertreter-Marketing erfordert einen einheitlichen Kern im Leistungsangebot, der gegenüber beiden Hauptzielgruppen zum Tragen kommt. Die grundsätzliche Möglichkeit einer einheitlichen Gestaltung des Dienstleistungskerns wurde durch die empirischen Resultate mehrfach belegt, da die Erwartungen der Marktpartner in den Hauptzielgruppen tendenziell gleichgerichtet waren.

Das beschriebene Grundgerüst eines Dienstleistungsangebotes kann in einem weiteren Schritt Ausgangspunkt segmentspezifischer Anpassungsmaßnahmen sein. Diese Anpassungsmaßnahmen werden bei zusätzlicher Bearbeitung der übrigen Segmente erforderlich. In jedem Falle ist eine a-priori Einengung auf diese Randzielgruppen $z u$ vermeiden (Segmente I und II auf Hersteller- sowie II und III auf Kundenebene). Ein konzentriertes Marketing gegenüber Randsegmenten engt auf seiten der genannten Herstellergruppen die Entfaltungsmöglichkeiten eines umfassenden und insbesondere eigenständigen Dienstleistungsangebotes ein. Die alleinige Bearbeitung der Randgruppen auf Kundenebene ist in einem Fall wegen mangelnder Segmentgröße, im anderen Fall wegen der drohenden Abhängigkeit von Großabnehmern wenig sinnvoll.

Nach den vorliegenden Resultaten eignen sich beide Randgruppen auf Hersteller und Kundenseite als ergänzende Marktsegmente, deren Bearbeitung eine sorgfältige Abwägung potentieller Chancen und Risiken in stärkerem Maße als bei den Hauptzielgruppen erforderlich macht. 
Ein solchermaßen integriertes Handelsvertreter-Marketing formt die strategische Basis zur langfristigen Absicherung der Position von Handelsvertretungen zwischen Herstellern und Abnehmern. Ein zielgruppengerechtes Dienstleistungsangebot trägt darüber hinaus zur wirksamen Abgrenzung von den unmittelbaren Konkurrenten bei. Dies gilt in besonderem Maße gegenüber den Reisenden, die i.d.R. über kein derart umfassendes Angebot an Dienstleistungen verfügen.

Eine Profilierung ist ferner gegenüber anderen Handelsvertretungen angezeigt. Diejenigen Handelsvertretungen können Wettbewerbsvorteile für sich verbuchen, die es verstehen, auf die individuellen Wünsche und Bedürfnisse unterschiedlicher Zielgruppen einzugehen ${ }^{1}$. Dies gilt sowohl für den zunehmenden Wettbewerbsdruck auf der Kundenseite als auch für die Akquisition geeigneter Vertretungen auf Seiten der Hersteller.

Letztendlich muB das integrierte Handelsvertreter-Marketing die vertragliche Verantwortung gegenüber den Herstellern und die informelle verantwortung gegenüber den Abnehmern gleichermaßen berücksichtigen. Diese dualistische Ausrichtung mündet in die Forderung an Handelsvertreter, "beim Kunden mit Nachdruck für die Interessen der vertretenen Firmen und bei den vertretenen Firmen mit Nachdruck für die Kunden einzutreten" ${ }^{2}$.

1 Eine starke Zunahme derartigen Marketingdenkens auf seiten der Handelsvertretungen betont Tietz, B., a.a.0., S.118.

2 Voss, H., Wandel durch Nachdenken, a.a.O., S. 10 . 


\section{Anhang I}

\section{Tabellen}

Tab. 1: Ausprägungen der Bestimmungsfaktoren der Rollenausübung von Handelsvertretungen

Tab. 2: Ausprägungen der Rollenausübung von Handelsvertretungen in allen Dienstleistungsbereichen

Tab. 3: Ausprägungen der Bestimmungsfaktoren von Rollenerfüllung und -erwartung bei den vertretenen Unternehmen

Tab. 4: Ausprägungen der Rollenerfüllung von Handelsvertretungen im Urteil der vertretenen Unternehmen

Tab. 5: Ausprägungen der Rollenerwartungen an Handelsvertretungen im Urteil der vertretenen Unternehmen

Tab. 6: Ausprägungen der Rollenerfüllung von Handelsvertretungen im Urteil der Abnehmer

Tab. 7: Ausprägungen der Rollenerwartungen an Handelsvertretungen im Urteil der Abnehmer

Tab. 8: Ausprägungen der Bestimmungsfaktoren von Rollenerfüllung und Rollenerwartung auf Kundenebene

Tab. 9: Segmentierung des Herstellermarktes (2er, 3er und 4er-Lösung)

Tab. 10: Dienstleistungsbezogene Segmentstruktur auf Herstellerebene

Tab. 11: Demographische Segmentstruktur auf Herstellerebene

Tab. 12: Segmentierung des Kundenmarktes (2er, 3er und 4 er-Lösung)

Tab. 13: Dienstleistungsbezogene Segmentstruktur auf Kundenebene

Tab. 14: Demographische Segmentstruktur auf Kundenebene 


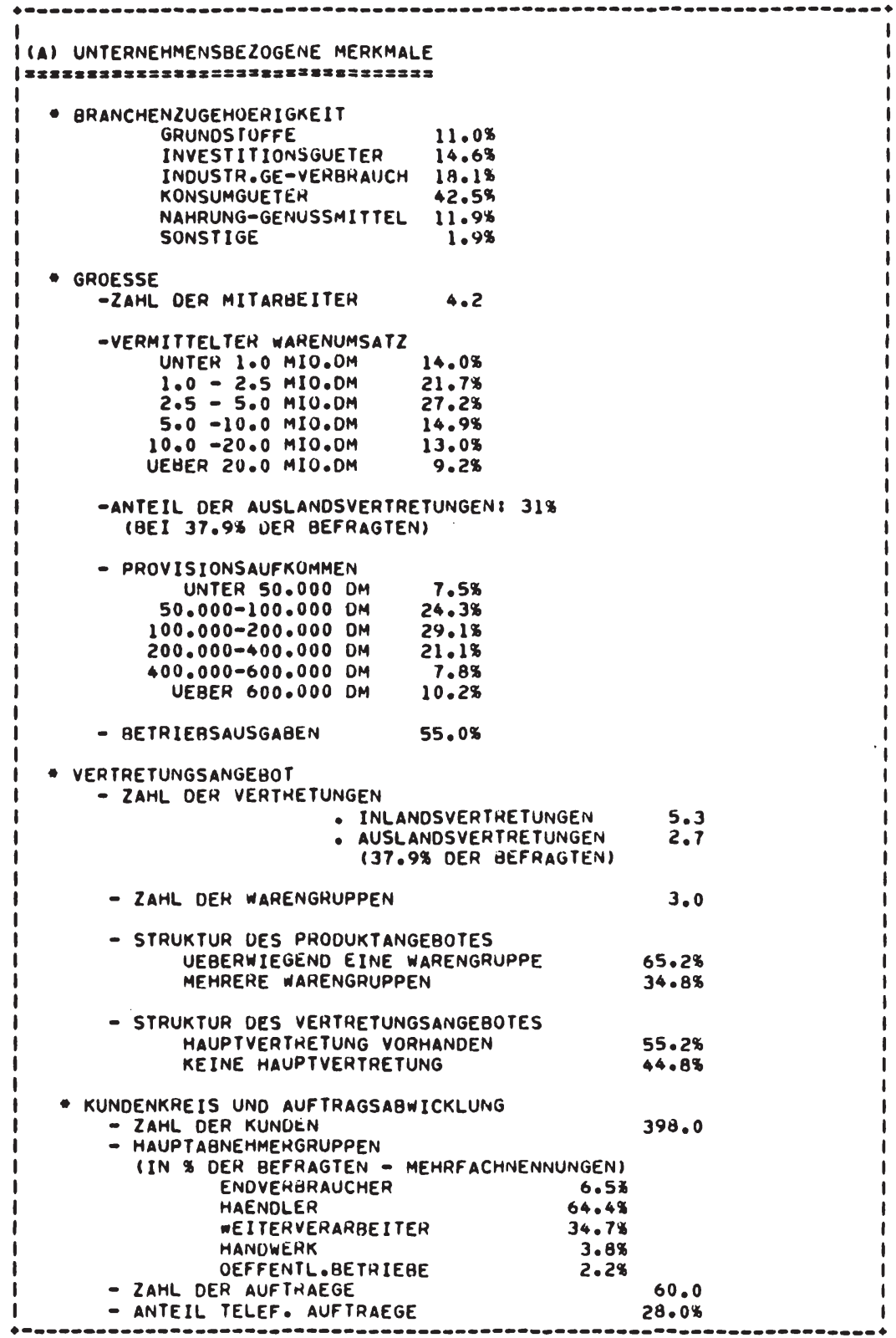

TAB. I: AUSPRAEGUNGEN DER BESTIMMUNGSFAKTOREN UER ROLLENAUSUEBUNG VON HANDELSVERTRETUNGEN (TEILI) 
- stanourt

- bUNDEsLANU

BERL IN

HAMBUNG

BREMEN

SCHLESWIG-HOLSTEIN

NIEOEHSACHSEN

NORORHE IN-WESTFALEN

HESSEN

RHE INLANU-PFALZ

SAARLANO

GADEN-WUERTTEMBERG

BAYERN

$3.6 \%$

$11.1 \%$

1.98

$3.1 \%$

$11.3 \%$

$32.0 \%$

$8.7 \%$

$2.2 \%$

$0.2 \%$

$13.9 \%$

- GeBIETSSTHUKTUR

12.08

(IN $\%$ DER BEFHAGTEN - MEHRFACHNENNUNGEN)

GROSSFLAECHIG

$49.0 \%$

MITTEL

BALLUNGSZENTREN

$44.4 \%$

$26.5 \%$

- allgemeine merkmale

- alter des UNTERneHMens (Jahre) 30.0

- ORTSGROESSE DES FIRIAENSITZES

$\begin{array}{rr}\text { UNTER } 10.000 & 13.1 \% \\ 10.000-50.000 & 21.5 \% \\ 50.000-100.000 & 7.4 \% \\ 100.000-250.000 & 12.2 \% \\ 250.000-500.000 & 8.1 \% \\ 500.000-\quad 1 M 10 & 19.6 \% \\ \text { UEBER IMIO } & 17.9 \%\end{array}$

(B) PERSONENHEZOGEIVE MERKMALE

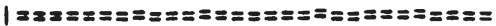

- geschlecht

MAENIVLICH $98.6 \%$

WEIBLICH $1.4 \%$

- alter 50.2

- ausailloung

KAUFM. LEHRE $67.6 \%$

TECHN. LEHRE 10.6\%

FACHHOCHSCHULE 15.7\%

UNIVERSITAETSSTUDIUM 6.1\%

- daver der berufstaetigkeit (Jahre) 20.0

- stellung im uivterivehmen
ALLE IHINHABEK
81.38
MITIINHABER
13.48
GESCHAEF ISFUEHRER
$5.0 \%$

TAB. 1: AUSPRAEgUnGEN UER hest IMMUNGGFAKTOREN DER ROLLENAUSUEBUng VON HANDELSVERTHETUNGEN (TEILZ) 
HOCH

$\bullet$

NIEOHIG

INF OKMATIONSAUSTAUSCH

VERWERTUNG HERSTELLER INFURMATIONEN VERWERTUNG KUNDENINFORMATIONEN GENUEGEND INFORMATIONEN AN KUINDEN KENNITNIS DER UMSATZKUNUENSTRUKTUR AUSREICHEND BERICHTE AN HERSTELLER AKTUALISIERTE KUNUENKARTEI HERSTELLERINFORMATION UEGER PLANUNGEN ERWEITERUNG UES KUNDENKREISES

INNERAETRIEBLICHE LEISTUNGSFAEHIGKEIT

QUERO PERSUNELL GESETLT

BUERO HAT TECHN. HILFSMITTEL

QUALIFIZIERTE MITAKBEITER

LANGFRISTIGER KUNUENKONTAKT

PREISGESTALTUNG

HANDLUNGSSPIELRAUM FUER PREISNACHLAESSE ERMESSENSSPIELRAUM FUER KONOITIONEN

\section{PROOUKTANREGUNGEN}

BEE INFLUSSUNG HERSTELLERSORTIMENT ANREGUNGEN FUER NEUE PRODUKTE

\section{LEISTUNGSVERGUETUING}

KALKULATION PROVISIONSSAETZE

PROVISIONSVORSCHLAG

GESONOERTE VERGUETUNG ZUSAETZL. LEISTUNG

\section{VERTRETUNGSPHOGRAMM}

HERSTELLER ERGAENZEN SICH GUT VERTRETUNGSPROGRAMM NACH MARKTFORSCHUNG

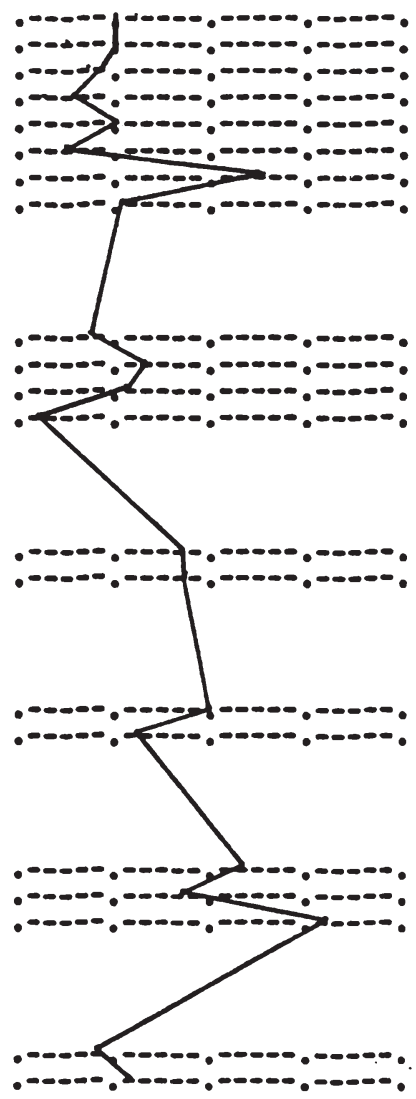

TAB. 2: AUSPRAEGUNGEN UEH ROLLENAUSUEBUNG VON HANDELSVERTRETUNGEN IN ALLEN DIENSTLEISTUNGSBERE ICHEIN 


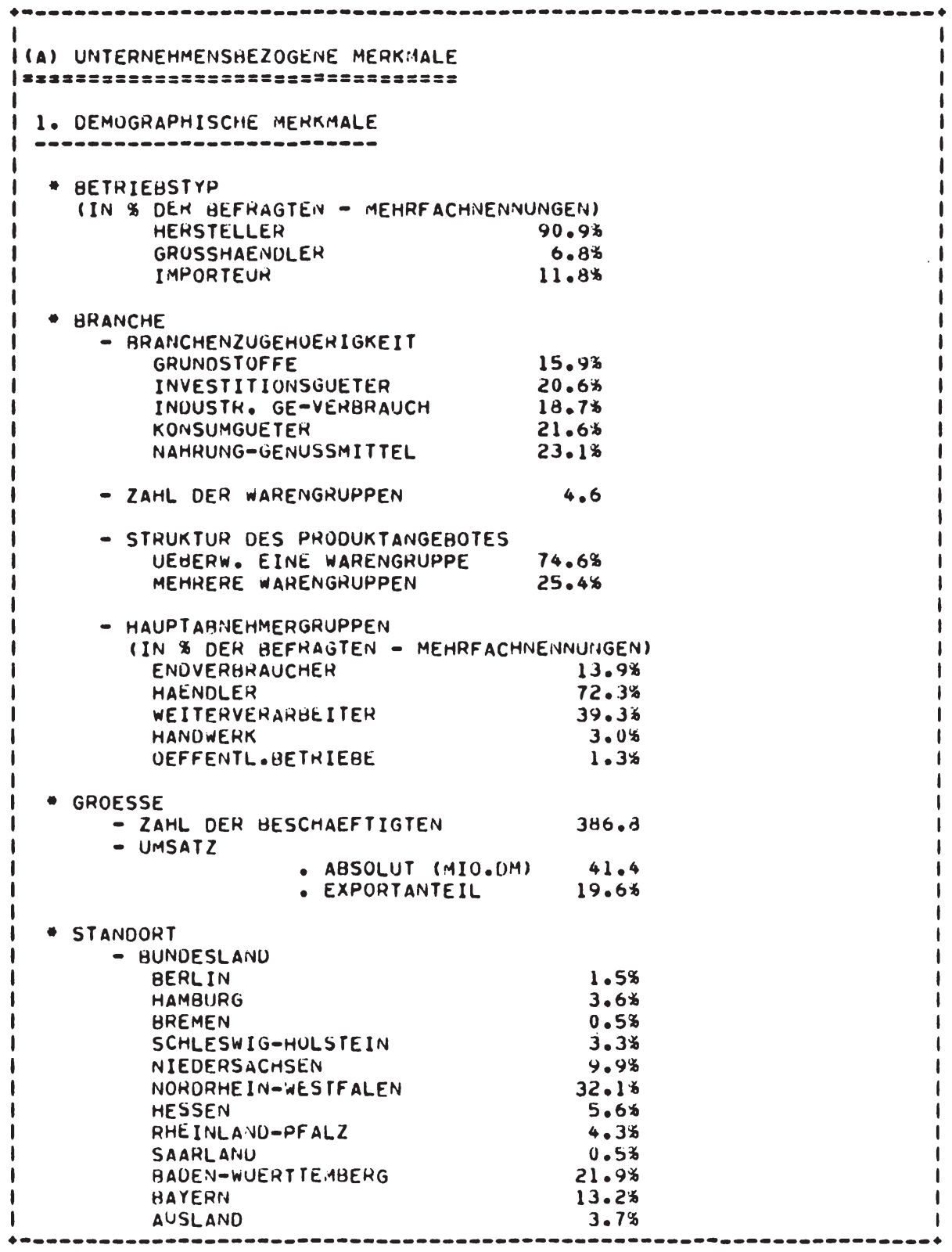

TA8. 3 : AUSPRAEGUNGEN UER BESTIMMUUGSFAKTUREN VON ROLLENERFUELLUNG UND -ERWARTUNG GEI UEN VERTRETEREN UNTERINEHMEN (TEILI) 


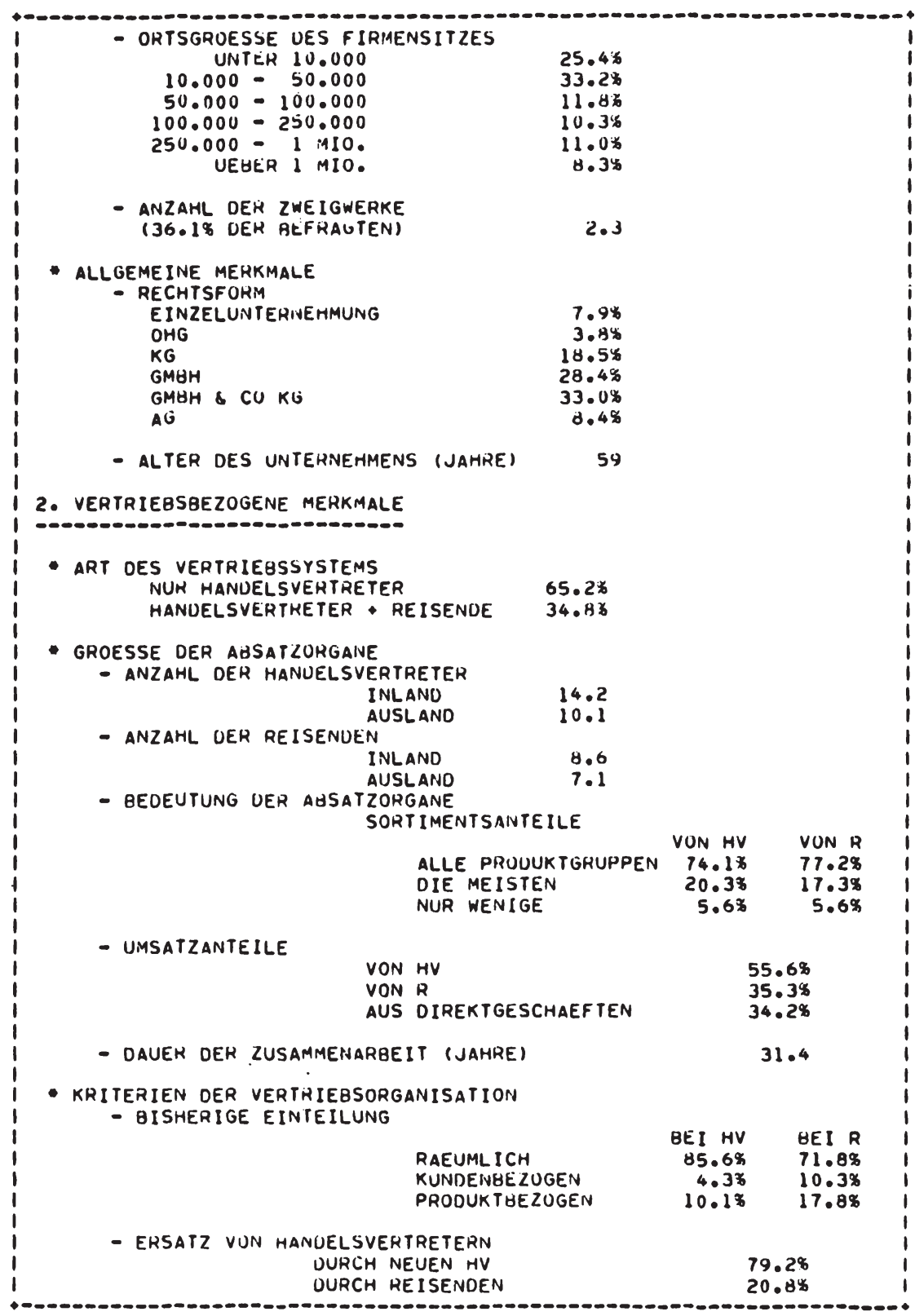

TAB. 3: AUSPRAEGUNÜEN UER BESTIMMUNGSFAKTOREN VUN ROLLENERFUELLUNG UND -ER IARTUNG UEI DEN VERTRETENEN UNTERNEHMEN (TEILZ) 
- rontaktaufnamme mit neUem hanuelsvertreter EMPFEHLUNG BISHERIGEH HV EIGENE ANZEIGEN BERUF SVERBAND KUNUENEMPFEHLUNG

- logistische merkmale

- ZAHL DER LAEGER

(GO\% DER BEFRAGTEN) 9.2

- lagerhalter

UNTERINEHMEN SELAST $19.5 \%$

SPEDITIONEN $14.5 \%$

HANUELSVEKTRETER $\quad 66.0 \%$

- zahl der hu-laeger 5.1

(B) PERSONENGEZOGEINE MERKMALE

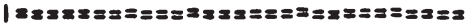

I

1. DEMOGRAPHISCHE MERKMALE

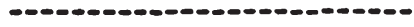

- geschlecht

MAENNLICH

WEI ILICH

$98.5 \%$

$1.5 \%$

- alter (Jamre)

45.1

- ausaildoung

KAUFM. LEHRE

TECHN. LEHRE

FACHHUCHSCHULE

49.96

UNIVERSITAETSSTUDIUM $22.9 \%$

- stellung im uivternehmen

ALLEIN-/MITINHABER 25.2\%

GESCHAEFTSFUEHRER $27.5 \%$

VERKAUFSLEITER $\quad 47.3 \%$

- dauer oer unternehiensZUGEHOERIGKEIT (JAHRE)

14.8

- oauer der positionsausuebung 11.2

2. PERSOENLICHE ZUSAMMENARBEIT MIT HV

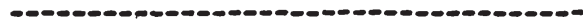

- anzahl oer pehsuenlichen kontakte (PRO MONAT)

23.8

- anteil der arueitszeit

$19.0 \%$

TAB. 3: AUSPRAEGUNGEN UEH HESTIMMUNGSFAKTUREIN VUN ROLLENERFUELLUNG UND -ERWARTUNG BEI DEN VERTRETENEN JIJTERNEHMEIN (TEIL3) 
INF ORMATIONSVERARBE ITUING

VERWERTUNG HERSTELLERIINF ORMATIONEN VER WERTUNG KUNDENIINF ORMATIONEN GENIJEGEND INFORMATIUNEN AN KUNDEN

KUNDENBE TREUUNG

QUAL IFIZIERTE MITARBEITER AKTUALISIERTE KUINUEIIVKAKTEI LANGFRISTIGER KUNDENKONTAKT

ERWEITERUNG DES KUNDENKREISES KENNTNIS DER UMSATZKUNUENSTRUKTUR MERSTELLER ERGAENZEN SICH GUT

\section{PRODUKTANREGUNGEN}

ANREGUNGEN FUER NEUE PROOUKTE BEEINFLUSSUNG HERSTELLERSORTIMENT VERTRETUNGSPROGRAMM NACH MARKTFORSCHUNG

PREISGESTALTUNG UNO LEISTUNGSVERGUETUNG ERMESSENSSPIELRAUM FUER KONOITIONEN HANDLUNGSSPIELRAUM FUER PREISNACHLAESSE GESONDERTE VENGUETUNG LUSAETZL. LEISTUHG PROVISIONSVORSCHLAG

KALKULATION PROVISIUNSSAETZE

AUSSTATTUNG DER HAINOELSVERTRETUNG

BUERO PERSONELL GESETZT

BUERO HAT TECHN. HILFSMITTEL

INFORMATION AN DEN HERSTELLER

AUSREICHEND BERICHTE AN HERSTELLER HERSTELLERINFORMATIUN UEBER PLAINUNGEN

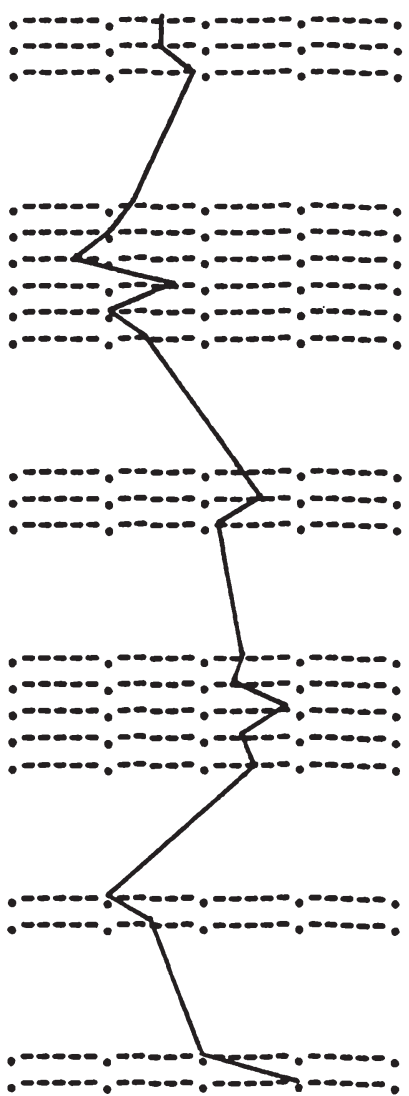

TAB. 4 : AUSPRAEGUNGEN DER ROLLENERFUELLUNG VON HANDELSVERTRETUNGEN IM URTEIL UER VERTRETENEN UNTERINEHMEN 


\section{KUNOENBETHEUUNG}

QUALIFIZIERTE MITARAEITER AKTUALISIERTE KIJPJEIVKAKTEI KENNTNIS DER UMSATZKUNDENSTRUKTUR LANGFRISTIGER KUNDENKONTAKT ERWE ITERUNG DES KUIVOENKREISES HERSTELLER ERGAENZEN SICH GUT

\section{INF ORMATIONSAUSTAUSCH}

VERWERTUNG HERSTELLERINF ORMATIONEN VERWEHTUNG KUNDENINFORMATIUNEN GENUEGEND INFORMATIONEIN AN KUNOEN MERSTELLERINFORMATIUN UEOER PLANUNGEN AUSREICHEND BERICHTE AN HERSTELLER

PRODUKTANREgUINGEN

BEEINFLUSSUNG HERSTELLERSORTIMENT ANREGUNGEN FUER NEUE PKOUUKTE VERTRETUNGSPROGRAMIY NACH MARKTFORSCHUNG

\section{PREISGESTALTUNG}

ERMESSENSSPIELRAUM FUER KONDITIONEN HANOLUNGSSPIELRAUM FUER PREISNACHLAESSE GESONOERTE VERGUETUNG ZUSAETZL. LEISTUNG

\section{AUSSTATTUNG DER HANUELSVERTRETUNG}

BUERO PERSONELL BESETZT

BUERO HAT TECHN. HILFSIMITTEL

\section{LEISTUNGSVERGUETUNG}

KALKULATION PROVISIONSSAETZE PROVISIONSVORSCHLAG

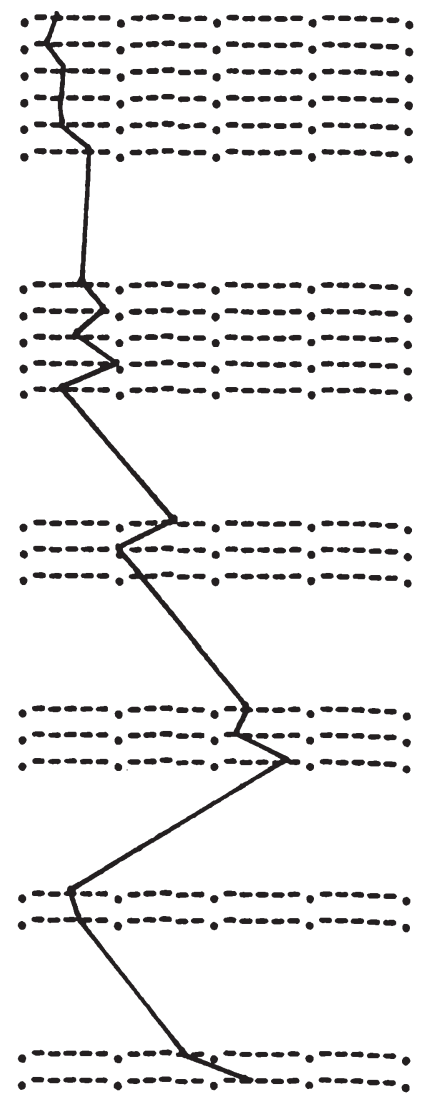

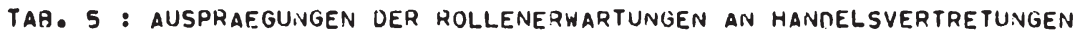
IM URTEIL DER VERTRETENEN UNITERNEHMEN 


\section{PRODUKTANREGUNGEN}

ANREgUNGEN FUER NEUE PRODUKTE BEE INFLUSSUNG HERS TELLERSORTIMENT

VEFWERTUNG KUNDENINFORMATIONEN VERTRETUNGSPROGRAMM NACH MARKTFORSCHUNG

\section{PREISGESTALTUNG}

ERMESSENSSPIELRAUM FUER KONDITIONEN HANDLUNGSSPIELRAUM FUER PREISNACHLAESSE

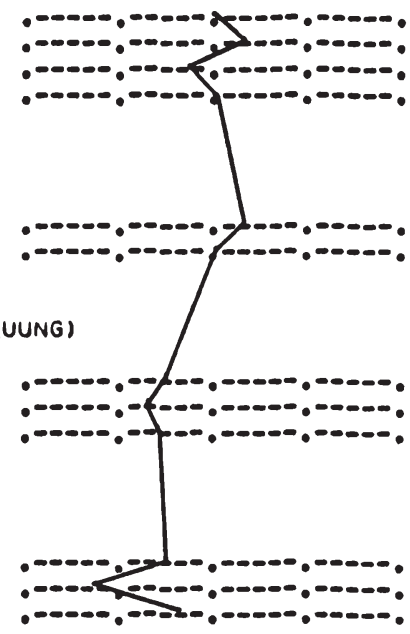

INNERBETR • LEISTUNGSFAEHIGKEIT (KUNDENEETREUUNG)

BUERO HAT TECHN. HILFSMITTEL

BUERO PERSONELL BESETZT

QUALIF IZIERTE MITARBE ITER

\section{VERTRETUNGSPROGRAMM}

HERSTELLER ERGAENZEN SICH GUT LANGFRISTIGER KUNDENKONTAKT GENUEGENO INFORMATIONEN AN KUNDEN

TAB. 6: AUSPRAEGUNGEN DER RULLENERFUELLUNG VON HANOELSVERTRETUNGEN IM URTEIL DER ABIVEHMER

\section{PRE ISGESTALTUNG}

ERMESSENSSPIELRAUM FUER KONOITIONEN HANOLUNGSSPIELRAUM FUER PREISNACHLAESJE

\section{PROOUKTANREGUNGEN}

ANREGUNGEN FUER NEUE PROOUKTE BEE INFLUSSUNG HERSTELLERSORTIMENT VEHTRETUNGSPROGRAMM NACH MARKTFORSCHUIVG VERWERTUNG KUNDENINFORMATIONEN

INNERBETR. LEISTUNGSFAEHIGKEIT (KUNDENGETREUUNG)

BUERO HAT TECHN. HILFSMITTEL BUERO PERSUINELL BESETZT

OUALIFIZIEHTE MITARUEITER

VERTHETUNGSPRUGRATM

HERSTELLER ERGAENZEIV SICH GUT GENUEGEND INFORMATIONEN AN KUNDEN LANGFRISTIGER KUIVISENIKUNTAKT

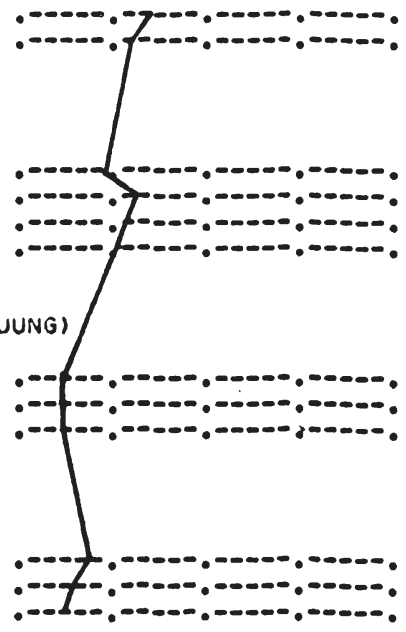

TAE. 7: AUSPRAEGUNGEN DER ROLLENERWARTUNGEN AN HANOELSVERTRETUNGEN IA UKTEIL DER ABNEHMER 


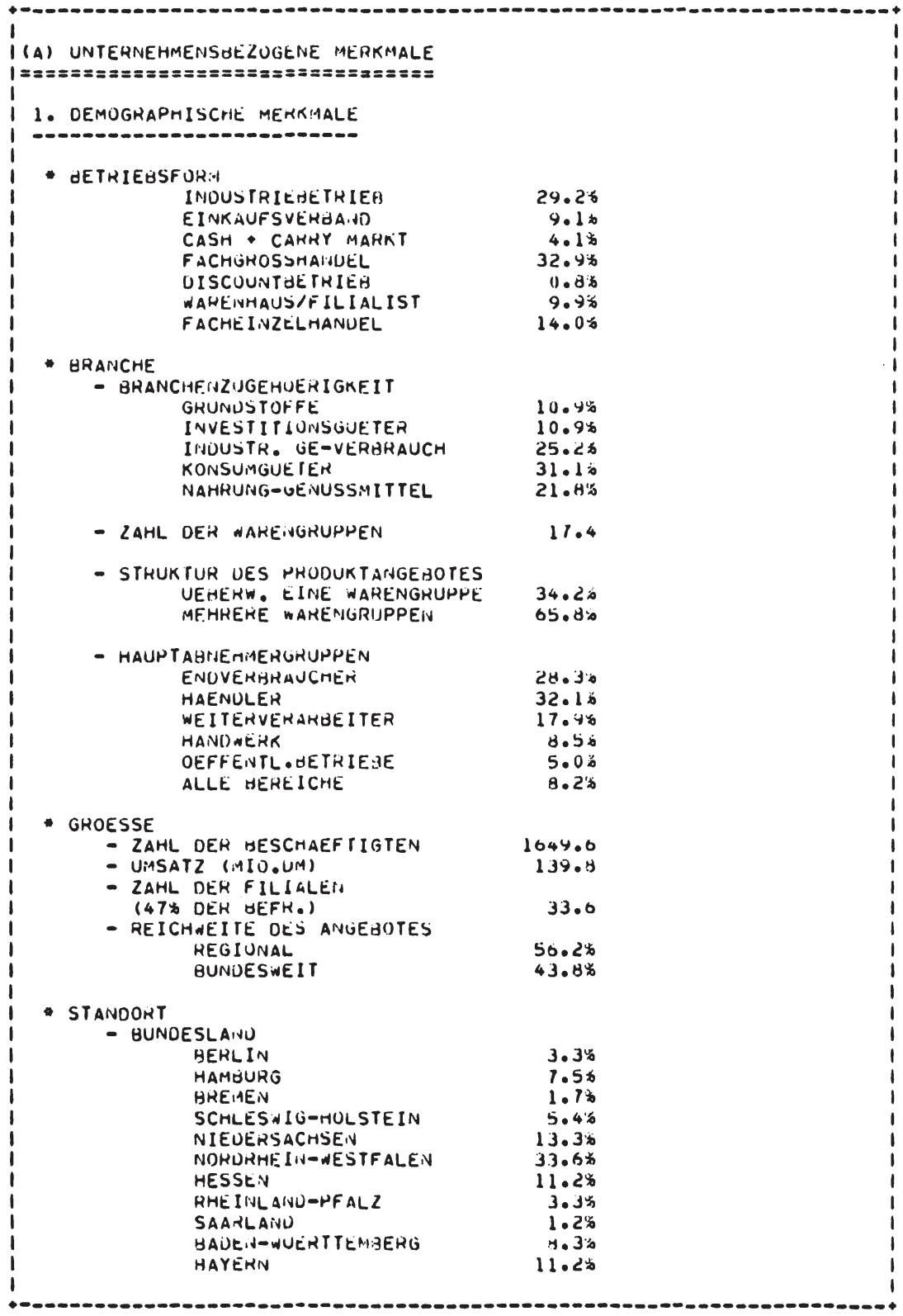

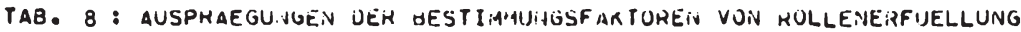
UIAI) RULLE.VERTARTUNG AUF KUNII)E'VCOEENE (TEIL l) 
- ORTSGHUESSE DES FIR:AENSITZES

$\begin{array}{rr}\text { UNTEH } 10.000 & 7.5 \% \\ 10.000-50.000 & 25.5 \% \\ 50.000-100.000 & 8.8 \% \\ 100.000-250.000 & 13.4 \% \\ 250.001-500.000 & 11.3 \% \\ 500.000-1.1100 & 16.7 \% \\ \text { UEBEH } 1110 . & 15.7 \%\end{array}$

- allgemejine mékKimale

- RECHTSFORIM

$\begin{array}{lr}\text { EINZELUNIERNEHMUNG } & 16.9 \% \\ \text { OHG } & 3.8 \% \\ \text { KG } & 19.2 \% \\ \text { GMGH } & 23.2 \% \\ \text { GMOH U. CO KG } & 24.9 \% \\ A G & 9.3 \%\end{array}$

- Alter des unternetMens (JAHRE) 50.0

2. EINKAUFSHEZOGENE MERKMALE

1

- einkaufsurganisatiun

- oRgaNISATIUNSKRITENIEN

NACH BELUGSOUELLEN $40.5 \%$

NACH EIGEINEM SORTIMEITT $48.1 \%$

NACH SPEL.PHODUKTMERKMALE'S $2.1 \%$

MEHRERE OER KRITERIEIN 9.3\%

- ANZAHL DER EINMAEUfER 8.6

- art der einkaufseintscheinung

$\begin{array}{lr}\text { EINKAEUFER ALLEIN } & 27.9 \% \\ \text { EINKAUFSLEITER ALLEIN } & 18.3 \% \\ \text { GESCHAEFTSFUEHHER ALLEIN } & 20.7 \% \\ \text { MEHRERE PERSUNEN } & 27.2 \% \\ \text { SUNSTIGE } & 5.9 \%\end{array}$

- auftragsauwickLUng PER telefuin 32.0\%

- oezugsouellen

- SORTIMENTSANTEILE

GESAMTES SORTIMENT

WESENILICHE TEILE

ERGAENZENUE TEILE

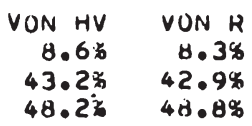

- einkaufsaivte ILE

HANOELSVERTRETER 18.0\%

NEISENUE 23.3\%

HERSTELLEH-DIREKTHEZUIj 54.0\%

ZEITTHALE 20.9\%

TAB. 8 : AUSPRAEGUNGEN DER BESTIMNUNGSFAKTOREN VON RULLENERFUELLUNG UND ROLLENERWAHTUNG AUF KUNOENEHENE (TEIL 2) 


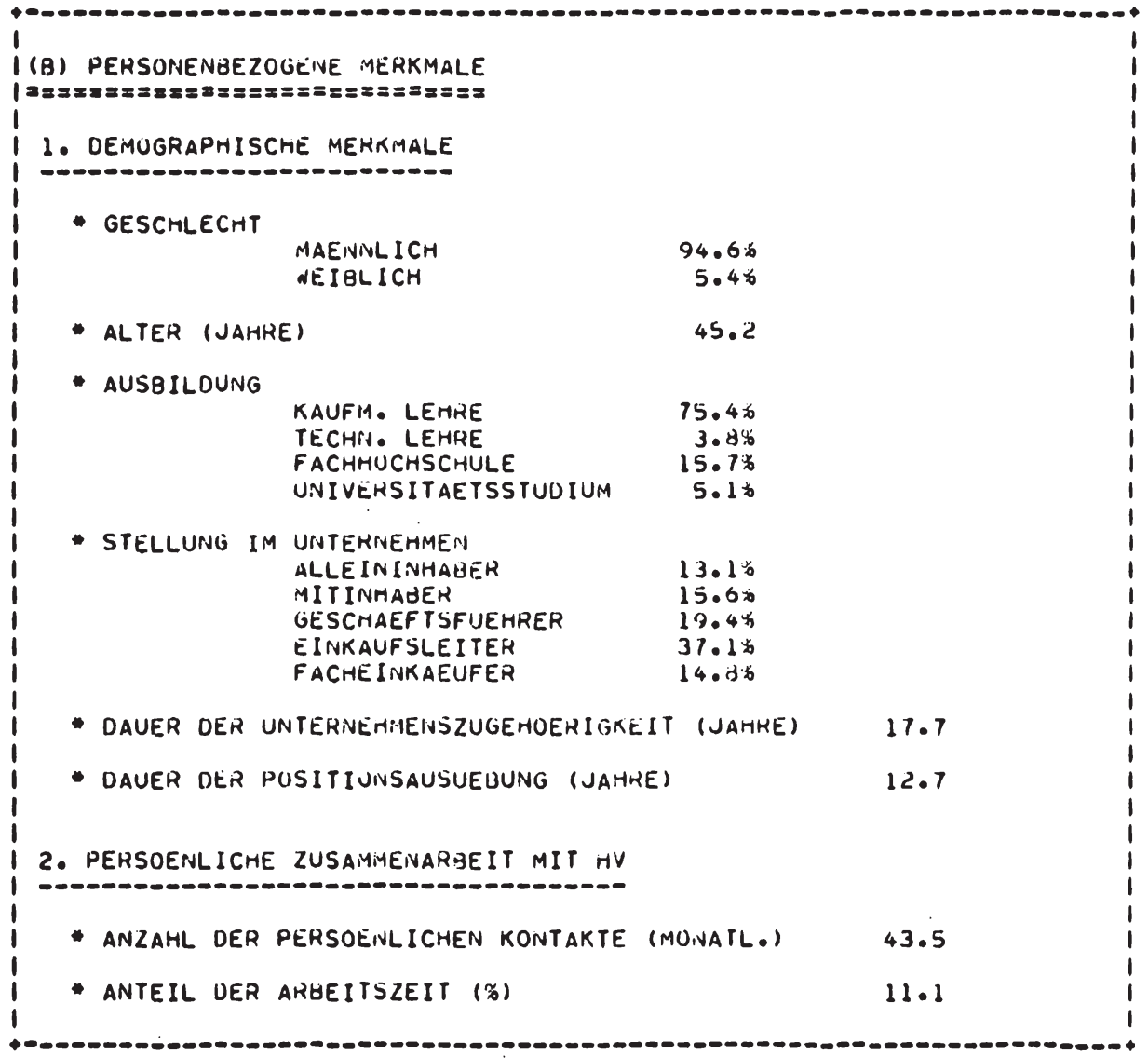

TAB. 8 : AUSPRAEGUIVGEN UER GESTIMMUNGSFAKTUREN VON RULLENERFUELLUNG UNO RULLEIVERWARTUNG AUF KUNOENEGEIVE (TEIL 3) 
CLUSTER-ANALYSIS

IHILL - CLIMAING - METHOUI

no of CASES In ANaLYSIS

402.

MO OF CLUSteas SPECHFIEUU

2

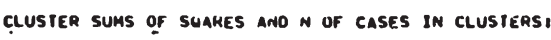

(1.00

1.6709

VARIANCE-CAITERION=

928.455

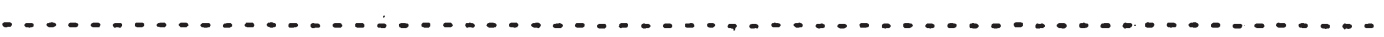

nO OF CASES IN ANALYSISI 402 .

NO OF CLUSTERS SPECIFIEDI 3

Cluster sUmS OF SOAHES AND $N$ OF CASES IN CLUSTERS,

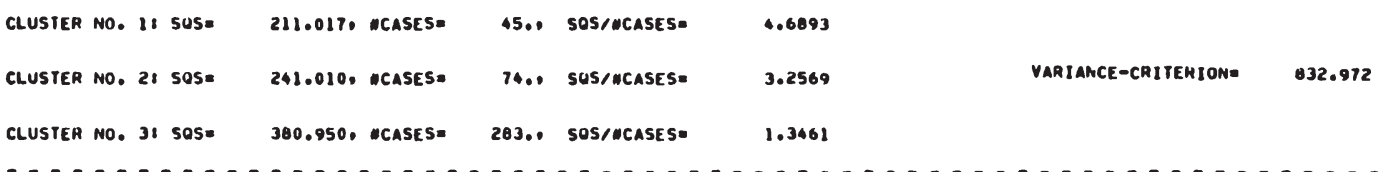

NO OF CASES IN ANALYSISI 402.

NO OF CLUSTERS SPECIFIEOI

CLUSTER SUMS UF SOARES AND N OF CASES IN CLUSTEKS:

\begin{tabular}{|c|c|c|c|c|c|c|c|c|}
\hline CLUSTER & NO. 11 SOS $=$ & 116.4590 & mCASES= & 46.0 & SUS/WCASES= & 2.5317 & & \\
\hline CLUSTER & NO. 21 SUS $=$ & 149.522 & WCASES= & 02.1 & SUS/WCASES= & 2.4116 & VARI ANCE - CR I TERION= & 753.364 \\
\hline CLUSTER & NO. 31 SOS= & 163.865 & MCASES = & 3н., & SOS/MCASES = & 4.3122 & & \\
\hline CLUSTER & NO. 4 SOS= & 323.581 , & "CASES" & 256.. & SUS/MCASES = & 1.2640 & & \\
\hline
\end{tabular}




\begin{tabular}{|c|c|c|c|c|c|}
\hline HVISTI & OUERU PERSUTELLL DESETZT & 2.01 & 2.70 & 1.88 & 1.93 \\
\hline MVIST2 & AUERO HAT TECHN. MILFSHITTEL & 2.40 & 3.27 & 2.36 & 2.27 \\
\hline HVIST3 & AKTUALISIEATE RUNUEHKAATEI & 2.03 & 2.33 & 1.92 & 2.01 \\
\hline HVIST4 & DUALIFIZIENTE MITAHEEITER & 2.21 & 2.42 & 2.22 & 2.17 \\
\hline HVISTS & LANGFHISTIGEN KUNUENKONTAKT & 1.71 & 1.82 & 1.76 & 1.60 \\
\hline MVISTOO & ERWEITEHUINL DES KUNUENKREISES & 2.74 & $2.9 n$ & 2.75 & 2.70 \\
\hline HVISTI & KENNINIS OEH UYSATLKUNOENSTAUKTUA & 2.05 & $2.1 \mathrm{~A}$ & 2.03 & 2.03 \\
\hline nVISTA & VERTRETUNOSPPUGGAMM NACH MARKTFURSCHUING & 3.13 & 3.3月 & 2.96 & 3.13 \\
\hline MVISTg & AMAEGUNGEIN FUEM NEUE PROUUKTE & 3.29 & 3.62 & 3.2 A & 3.24 \\
\hline HVISTIO & BEE INFLIJSSU'JU MERSTELLERSORTIMEIVT & 3.56 & 3.80 & 3.50 & 3.32 \\
\hline HVISTII & MERSTELLEN ERUAENZEN SICH GUT & 2.35 & 2.51 & 2.23 & 2.30 \\
\hline HVISTI2 & PRUVISIONSVORSCHLAU & 3.37 & 3.62 & $3.5 B$ & 3.27 \\
\hline nVIST13 & KALKULATIUN PHUVISIONSSAETZE & 3.50 & 3.80 & 3.45 & 3.46 \\
\hline HVISTI4 & GESONOERTE VERGUETUNG ZUSAETZL. LEISTUNG & 3.88 & 4.00 &. .30 & 3.74 \\
\hline MVISTIS & HANOLUNGSSPIELAAUM FUEM PREISPIACHLAESSE & 3.30 & $3.4 ?$ & 3.96 & 3.11 \\
\hline MVISTI6 & ERMESSENSSPIELARAUM FUEH KOVUITIUNEN & 3.40 & 3.27 & 4.55 & 3.12 \\
\hline HVISTIT & AUSHEICHENU BENICHTE AN HENSTELLER & 2.90 & 3.22 & 3.09 & 2.93 \\
\hline HVISTI8 & MERSTELLERINFUMAATION UEBER PLAIVUNGEN & 3.95 & 4.04 & 3.96 & 3.93 \\
\hline HVISII9 & GENUEGENO INFURMATIONEN AN KUNUEN & 2.88 & 3.29 & 2.74 & 2.85 \\
\hline HVIST2O & VERWERTUNG MENSTELLER INFORMATIUNEN & 2.63 & 2.90 & 2.49 & 2.62 \\
\hline MVIST2I & VERWERTUNO KUNUENNINFORMATIONEN & 2.60 & 3.00 & 2.46 & 2.57 \\
\hline HVSOLLI & GUEAO PERSUNELL OESEIZT & 1.47 & 2.36 & 1.31 & 1.37 \\
\hline HVSOLLZ & BUEHU HAT TECNN. MILFSAITTEL & 1.59 & 2.44 & 1.42 & 1.40 \\
\hline HVSOLL:3 & AKTUALISIERTE KUNOENIKARTEI & 1.21 & 1.64 & 1.00 & 1.17 \\
\hline HVSOLL & OUALIFIZIEFTE MITAKIEITER & 1.31 & 1.60 & $1.1 \mathrm{~A}$ & 1.30 \\
\hline HVSOLLS & LANGFRISTIGEA KUNDENKUNTAKT & 1.36 & 1.51 & $1.3 n$ & 1.36 \\
\hline HVSOLL6 & ERAEITEHUIJÜ OES KUNOENKREISES & 1.39 & 1.71 & 1.32 & 1.36 \\
\hline HVSOLLT & KENNTNIS UER UMSATZKUNOENSTRUKTUR & 1.42 & 1.62 & 1.41 & 1.30 \\
\hline HVSOLLB & VERTAE TUNGSPROGRAMM NACH MARKTFOHSCHUHG & 2.11 & 2.27 & 2.12 & 2.08 \\
\hline HVSOLL & ANHEGUNGEN FUER NEUE PRODUKTE & 1.98 & 2.27 & 1.89 & 1.96 \\
\hline HVSOLLLO & BEEINFLUSSUNG MEHSTELLERSONTIMENT & 2.63 & 2.60 & 2.73 & 2.61 \\
\hline MUSOLLII & HERSTELLER ERGAENZEN SICH GUT & 1.74 & 2.04 & 1.55 & 1.14 \\
\hline MVSOLL12 & SCHLAGE PHUVISIUNSSAETZE VDR & 3.20 & 3. h4 & 3.50 & 3.17 \\
\hline HVSOLL13 & KALKULIERE PROVISIUNSSAETZE & 2.71 & 3.36 & 2.62 & 2.03 \\
\hline HVSOLLLA & GESUNOERTE VEHGUETUNG ZUSAETZL. LEISIUNG & 3.81 & 3.98 & 4.30 & 3.65 \\
\hline MVSOLLLIS & MANOLUNGSSPIELRAUM FUER PREISNACHLAESSE & 3.22 & 3.44 & 3.47 & 2.48 \\
\hline HVSOLL16 & ERMESSENSSPIELHAUM FUER KONOITIONEN & 3.31 & 3.18 & 4.70 & 2.96 \\
\hline MVSOLL17 & AUSREICMENID GEHICHTE AN MERSTELLEER & 1.45 & 1.76 & 1.39 & $1 . \bullet 2$ \\
\hline HVSOLLIA & INFORMIERE MERSTELLER UEAER PLMNUNGEN & 1.95 & 2.33 & 2.07 & 1.00 \\
\hline HVSOLL19 & GENUESENO INFUAMATIONEN AN RUNUEN & 1.56 & 2.18 & 1.51 & 1.47 \\
\hline HVSOLL2O & VERMERTE AËHSTELLEMINFOAMATIUNENA & 1.66 & 2.20 & 1.51 & 1.01 \\
\hline HYSOLL2I & VERMERTE KUNOER INFURMATIONEN & 1.90 & 2.46 & 1.05 & 1.80 \\
\hline
\end{tabular}




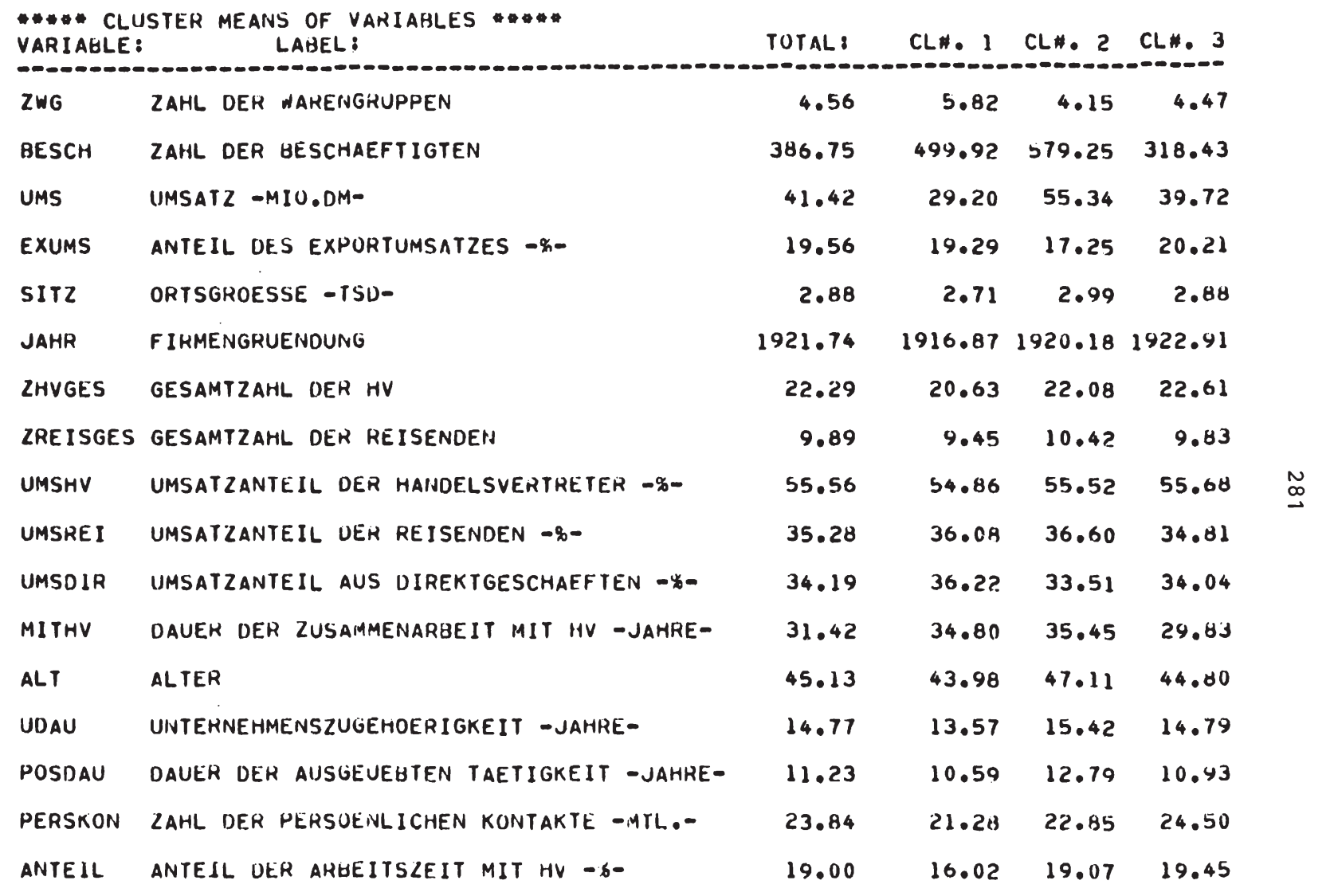

TAB. 11 : DEMOGRAPHISCHE SEGMENTSTRUKTUR AUF HERSTELLEREBEINE: 
NO OF CASES IN ANALYSISI

NU OF CLUSIEHS SPECIFIEUI

2

Cluster sUmS of SUAMES ANo $N$ OF CASES IN CLUSTERS,

CLUSTER NO. 11 SOSE 223.905 , ICASES= 203.1 SOS/MCASES= 1.1030 VARIANCE-CPITERION= 34B.4T7
CLUSTER NO. 21 SOSE

A2., SOS/WCASES: $2.966 \mathrm{~A}$

NO OF CASES IN ANALYSIS! 245.

No OF CLUSTERS SPECIFIEO

3

Cluster SUMS OF SOAHES AND $N$ OF CASES IN CLUSTERS,

\begin{tabular}{|c|c|c|c|c|c|c|c|c|}
\hline CLUSTER & NO. II SOS= & 125.411 , & mCAses= & 165.0 & SOS/WCASES" & 0.7601 & & \\
\hline CLUSIER & NO. 21 SUS $=$ & 107.913. & mCASES & $58 .$, & SOS/MCASES= & 1.8606 & VARIANCE-CRIIERION= & 296.786 \\
\hline CLUSIER & NO. 31 SOS= & 63.509 , & WCASES= & 22.0 & SOS/MCASES= & $2.886 \mathrm{~A}$ & & \\
\hline
\end{tabular}

NO OF CASES IN ANALYSIS:

245.

NO OF CLUSTEAS SPECIFIEU

4

Cluster SUMS OF SOAKES AND $N$ OF CASES IN CLUSTERS,

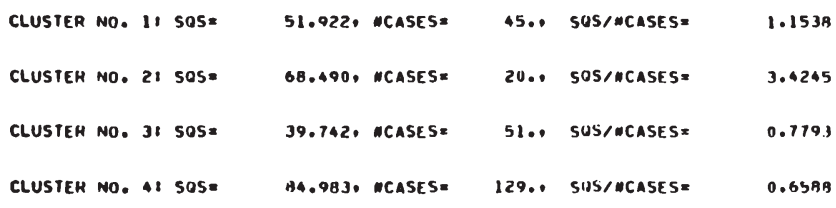

Tab. 121 SEGHENTIERUNG DES KUNDEMMARKTES (2ER-D JEH- UNU AEH-LOE SUNB) 


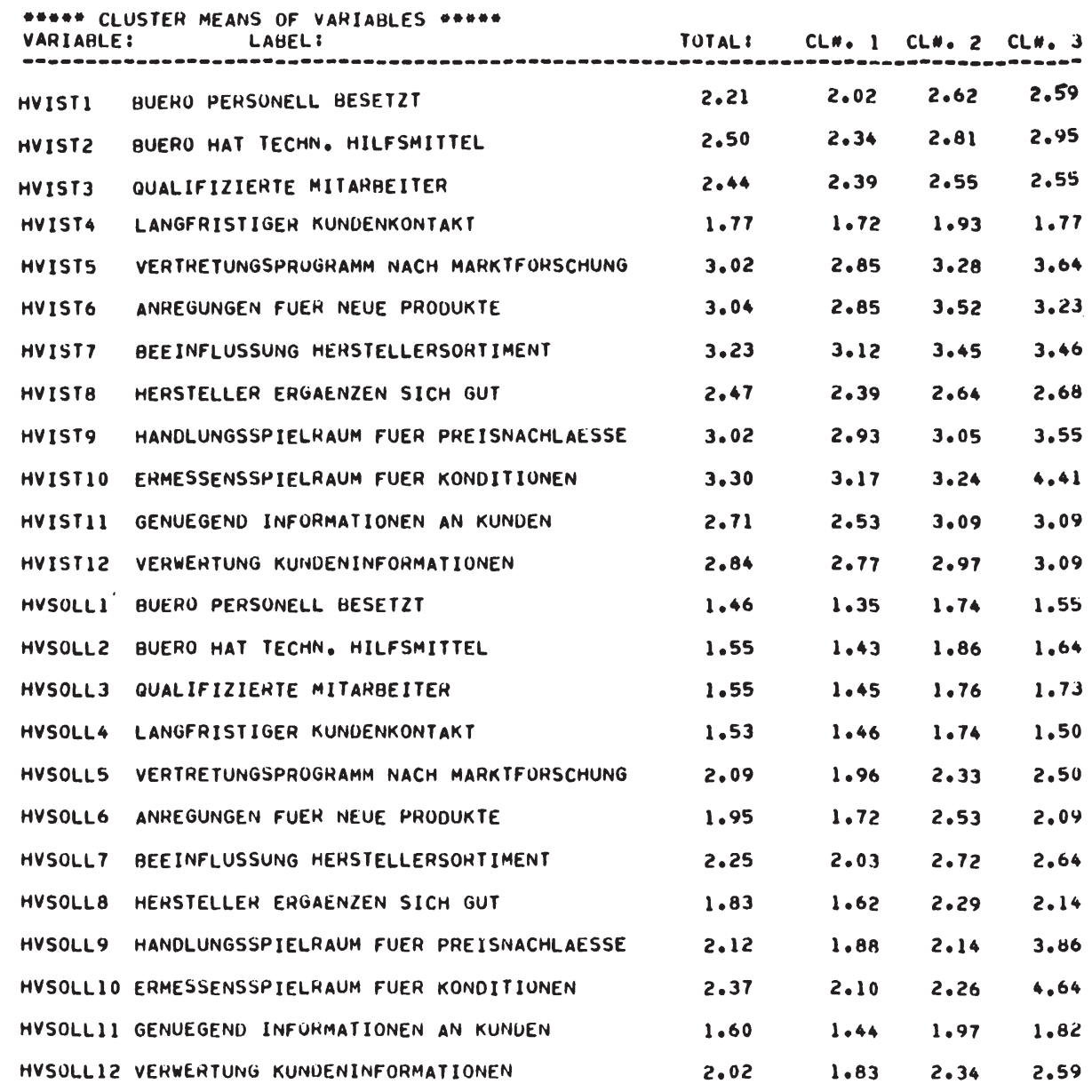

Tab. 13: DIENSTLEISTUNGSBEZOGENE SEGMENTSTRUKTUH AUF KUNDENEGENE 


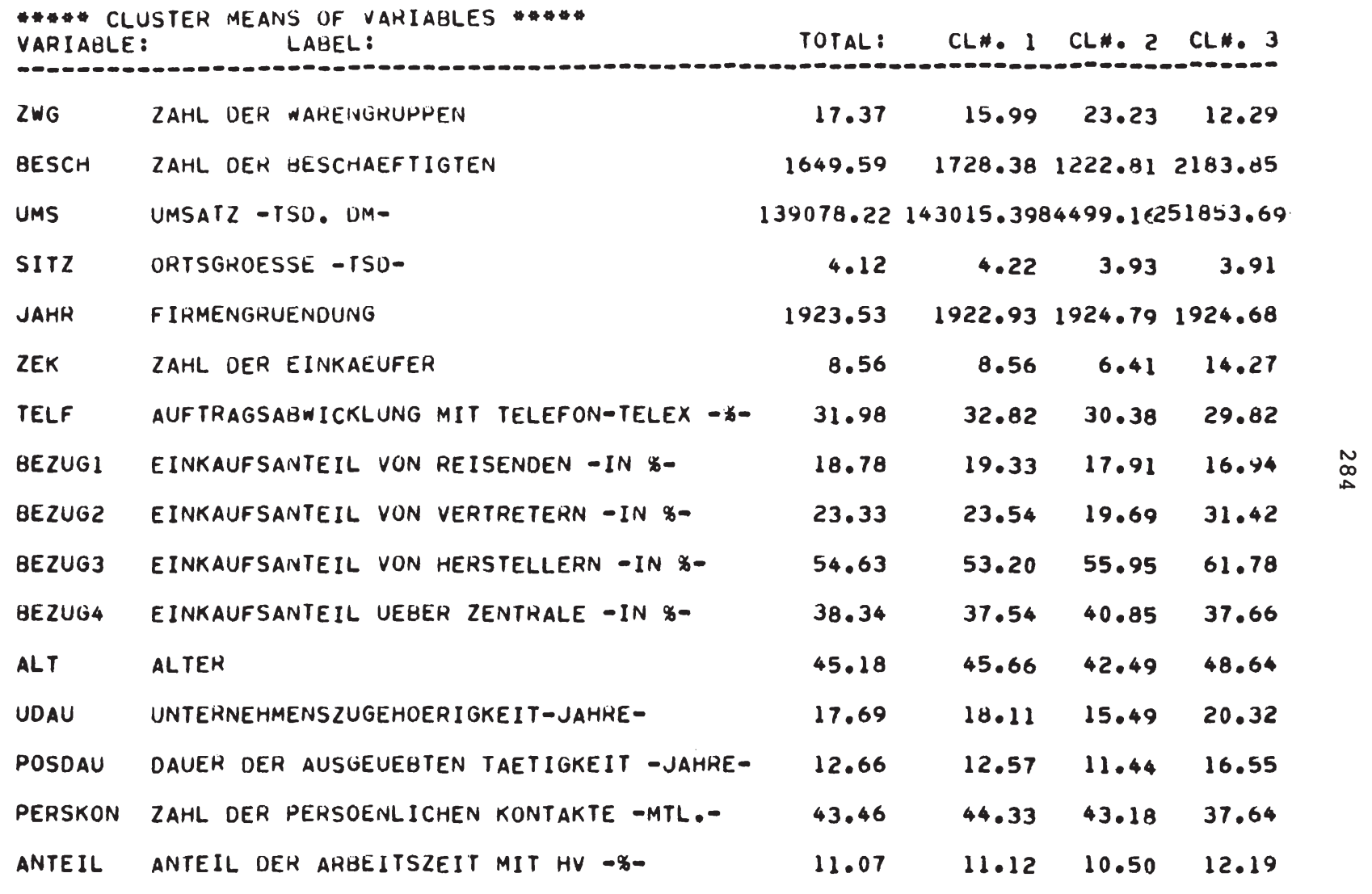


A $n \mathrm{~h}$ a $\mathrm{n} \mathrm{g}$

II

Fragebogen der Untersuchung 
Fragebogen

- Handelsvertreter ' 
In welchem Geschaftsbereich (Branche) sind sie Uberwlegend tatig?

Hauptgeschaffsbereich:

W1r nennen Ihnen $1 \mathrm{~m}$ folgenden elne Reihe von Tatigkeiten, die bel Ihrer tăglichen Arbeit als Handelsvertreter anfallen können. Bitte sagen Sie uns, wie nach Ihrer Einschatzung diese Aussagen im Durchschnitt für sie persönlich zutreffen (bitte den entsprechenden Punkt der Skala ankreuzen).

Mein Büro ist ständig personell besetzt.

Mein Buro 1st mit allen erforderlichen techn. Hilfsitteln ausgestattet (Telex, Anrufbeantw.).

Meine Handelsvertretung verfügt uber elne stăndg aktualisierte Kundenkarte1.

Die Qualifikation der Mitarbelter in meiner Handelsvertretung entspricht den Anforderungen.

Ich habe einen langfristigen Rontakt zu melnen Runden.

Ich erweitere melnen Kundenkrels ständig.

Ich we1B, wie sich mein gesamter Umsatz auf die verschledenen Kunden vertellt.

Be1 der zusammensetzung meines Vertretungssort1mentes stutze $1 \mathrm{ch} \mathrm{mich}$ auf elgene Marktuntersuchungen.

Ich gebe melnen vertretenen Unternehmen Anregungen fur die Entwicklung neuer Produkte.

Ich kann die Gestaltung des Sortimentes meiner vertretenen Unternehmen mit beeinflussen.

Die Produkte der verschiedenen Hersteller erganzen sich gut.

Vor VertragsabschluB werden die branchenublichen Provisionsautze von mir vorgeschlagen.

Vor der Ubernahme einer Vertretung fuhre $1 \mathrm{ch}$ elne Kalkulation meiner Provisionssatze durch.

zusatzliche Leistungen (z.B. Auslieferung, Regalpflege) stelle ich meinen vertretenen Unternehmen gesondert in Rechnung.

Be1 Verhandlungen mit Kunden habe 1 ch elnen Handlungsspielraum fur Preisnachlasse.

Be1 Verhandlungen mit Runden kann $1 \mathrm{ch}$ die Rond1tionen (z.B. Rabatte, Zahlungszlele) Innerhalb vorgegebener Bandbreiten nach elgenem Ermessen festlegen.

D1e vertretenen Unternehmen erhalten von mir in ausrelchendem MaBe aktuelle Berichte.

Ich informiere die vertretenen Unternehmen über melne eigenen Planungen (z.B. Umsatzzlele eines neuen Jahres).

Meinen Kunden blete $1 \mathrm{ch}$ genugend aktuelle Informationen.

Die Informationen der vertretenen Unternehmen verwerte ich für elgene Planungen und MaBnahmen.

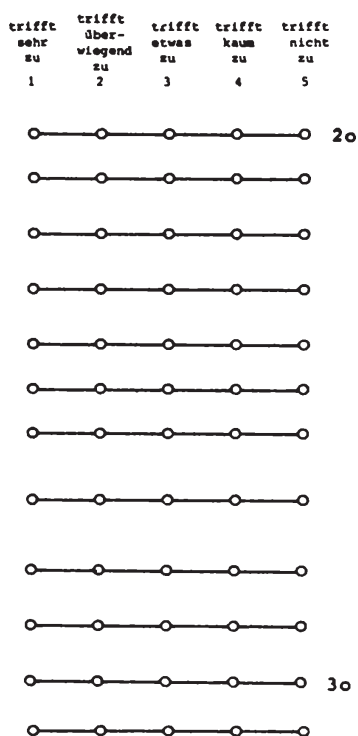

Die Informationen der Kunden verwerte 1ch fur elgene Planungen und Maßnahmen.
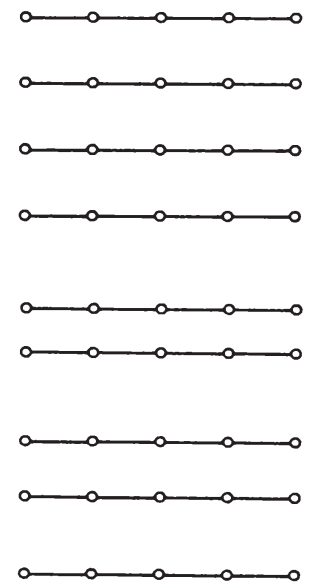
Abschließend bitten wir sie um elnige Informationen uber Ihre Handelsvertretung. Die Angaben werden - wie alle übrigen Antworten - selbstverständlich vertraulich behandelt.

Wie viele Vertretungen haben Sie?
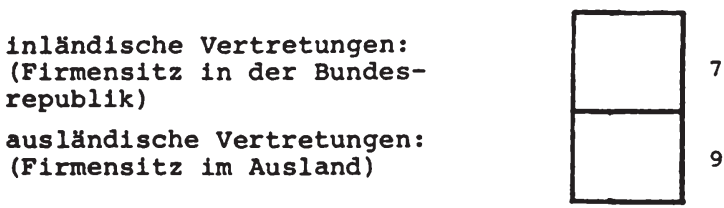

Betrachten sie bitte einmal Ihr gesamtes Vertretungssortiment. Konzentriert sich Ihr Vertretungsprogramm auf elne bestimmte warengruppe oder verkaufen sie eine Reihe von Waren, die sehr verschieden voneinander sind (wie z. B. Wein oder Subwaren/Schränke oder Tische)? (B1tte ankreuzen)

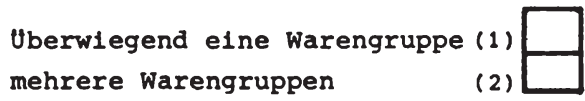

Wieviele solcher Warengruppen bieten Sie insgesamt an?

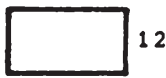

Wieviele dieser Warengruppen stammen aus ausländischer Produktion?

Anzah 1:

Wieviele Kunden betreut Ihre Handelsvertretung insgesamt? (evtl. Schätzung)

Kundenzahl :
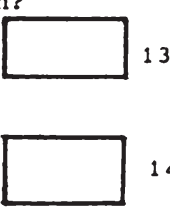

Betrachten Sie bitte elnmal Ihren gesamten Auftragseingang. Wie groB 1st der Antell der Auftrăge, die Sle uber Telefon (und Telex) mit Ihren Kunden abwickeln?

(evtl. Schätzung)

Auftragsabwicklung durch Telefon/Telex:

Wieviele Aufträge vermitteln Sie insgesamt im Durchschnitt während einer Woche?

(evtl. Schätzung) 
Wieviele Personen sind in Ihrer Handelsvertretung insgesamt tätig? (einschl. tatigen Inhabern und mitarbeitenden Familienangehörigen)

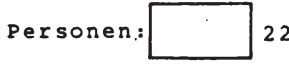

In welchem Jahr wurde Ihre Handelsvertretung gegründet?

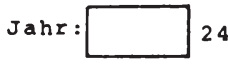

Wie groB ist der ort, in dem Ihre Handelsvertretung ihren Stammsitz hat?

\begin{tabular}{|ll|l|}
\hline unter 10.000 \\
\hline 10.000 - unter 50.000 & $(1)$ & \\
\hline 50.000 - unter 100.000 & $(3)$ & \\
\hline 100.000 - unter 250.000 & $(4)$ & \\
\hline $250.000-$ unter 500.000 & $(5)$ & \\
\hline 500.000 - unter 1000.000 & $(6)$ & \\
\hline uber 1.000 .000 & $(7)$ & \\
\hline
\end{tabular}

Welche Struktur hat das von Ihrer Handelsvertretung betreute Geblet?

\begin{tabular}{|l|r|r|}
\hline grobflachig, Kunden liegen weit auseinander (1) & \\
\hline mittlere Grobe, mehrere Kunden an einem Ort (2) & \\
\hline Ballungszentren & (3) \\
\hline
\end{tabular}

Haben Sie eine Hauptvertretung, wir meinen damit eine Vertretung, die mehr als 508 Ihrer gesamten frovisionseinnahmen erbringt?

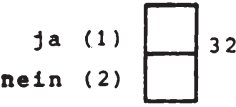

Wer sind Ihre Hauptabnehmergruppen?

\begin{tabular}{|lr|r|}
\hline Endverbraucher & (1) & \\
\hline Wiederverkäufer (Händler) & (2) & \\
\hline Welterverarbelter (Industriebetriebe) & (3) & \\
\hline Handwerksbetriebe & (4) & \\
\hline Offentliche Betrlebe & (5) & \\
\hline
\end{tabular}

Wie hoch war 1978 der von Ihrer Handelsvertretung vermittelte Warenumsatz? (nicht Provision, einschlieBlich Eigengeschafte, ohne MwST)

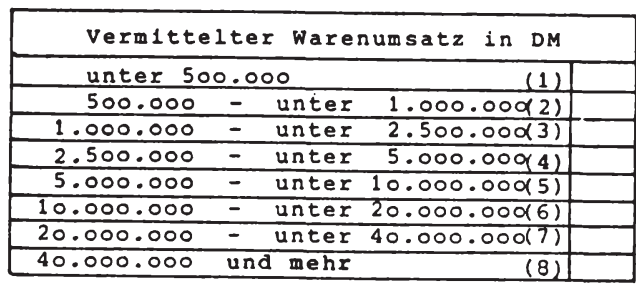

Welcher Umsatzanteil entfällt dabel auf die ausländischen Vertretungen? 
Wie hoch waren Ihre gesamten Bruttoeinnahmen in 1978? (Provisionen, zusatzliche vergutungen, Bruttoertrag aus Eigengeschaften, ohne MWST)

\begin{tabular}{|l|l|l|}
\hline \multicolumn{3}{|l|}{ Bruttoeinnahmen in DM } \\
\hline unter 25.000 & $(1)$ & \\
\hline $25.000-50.000(2)$ & \\
\hline $50.000-100.000(3)$ & \\
\hline $100.000-200.000(4)$ & \\
\hline $200.000-400.000(5)$ & \\
\hline $400.000-600.000(6)$ & \\
\hline $600.000-1000.000(7)$ & \\
\hline 1.000 .000 und mehr(8) & \\
\hline
\end{tabular}

Wie werden die Provisionen ausländischer Vertretungen abgerechnet? (b1tte ankreuzen)

mit der im Ausland ansässigen Firma direkt mit einer in der Bundesrepublik ansässigen Tochtergesellschaft/Niederlassung

Wie hoch 1st der Anteil Ihrer Betriebsausgaben an den gesamten Provisionseinnahmen?

(bitte in Prozent angeben)

In welchem Bundesland ist Ihr Unternehmen angesledelt?

$$
\text { Bundesland }
$$

Zum AbschluB gestatten Sie uns bitte einige Fragen zu Ihrer person.

sie sind

wie alt sind sie?

$$
\begin{aligned}
& \text { mannlich } \\
& \text { weiblich }
\end{aligned}
$$

Jahre

\begin{tabular}{|c|}
\hline kaufm. Lehre \\
\hline techn. Lehre \\
\hline $\begin{array}{l}\text { Fachhochschule } \\
\text { (2.B. Ing.grad.) }\end{array}$ \\
\hline $\begin{array}{l}\text { abgeschlossenes (4) } \\
\text { Universitatsstudium }\end{array}$ \\
\hline
\end{tabular}

Welche Berufsausbildung haben Sie?

Wie lange sind Sie bereits als Handelsvertreter tätig?

$$
\text { zahl der Jahre }
$$

Welche Stellung haben Sie in Ihrem Unternehmen? (bitte ankreuzen)

\begin{tabular}{|l|}
\hline Alleininhaber (1) \\
\hline Mitinhaber \\
\hline Geschäftsfuhrer(3) \\
\hline
\end{tabular}


Fragebogen

' Hersteller ' 
WESTF. WILHELMS.UNIVERSITATT

\section{INSTITUT FUR MARKETING \\ DIREKTOR: PROF. DR. H. MEFFERT}

\section{F R A G E B O G E N}

In welchem der nachfolgend genannten Geschäftsbereiche ist Ihr Unternehmen uberwiegend tätig?

(bitte eine Möglichkeit ankreuzen)

\begin{tabular}{|lr|l|}
\hline Stahl, Eisen, Halbfabrikate & $(11)$ & \\
\hline Papier und Pappen & $(12)$ & \\
\hline Garne & $(13)$ & \\
\hline Bauwesen & $(14)$ & \\
\hline Elektrotechnik & $(21)$ & \\
\hline Maschinen (einschl. Zubehör) & $(22)$ & \\
\hline Fahrzeuge & $(23)$ & \\
\hline Techn. Bedarf, Eisenwaren, Werkzeuge & $(31)$ & \\
\hline chemlsche Erzeugnisse, Kunststoffe, Lacke (32) & \\
\hline Sanitärsbedarf & $(33)$ & \\
\hline Verpackungsmittel & $(34)$ & \\
\hline Haushaltswaren (Glas, Keramik, Schmuck & $(41)$ & \\
\hline Textil, Bekleidung, Lederwaren & $(42)$ & \\
\hline Möbel & $(43)$ & \\
\hline Lebensmittel, SüBwaren & $(51)$ & \\
\hline Weine, Spirituosen & $(52)$ & \\
\hline
\end{tabular}

$\mathrm{Zu}$ welcher der nachfolgenden Gruppen zählt Ihr Unternehmen?

\begin{tabular}{|ll|l|}
\hline Hersteller & 11 & \\
\hline GroBhändler & 12 & \\
\hline Importeur von Handelswaren & (3) & \\
\hline
\end{tabular}

Welche der genannten Moglichkeiten unterhält Ihr Unternehmen in Vertrieb und Kundenbetreuung?

(bitte ankreuzen)

\begin{tabular}{|l|l|}
\hline Relsende im Verkauf & (1) \\
\hline Handelsvertreter & \\
\hline Innendienstsachbearbeiter ' & \\
die auch Kunden besuchen (3) & \\
\hline Techniker im AuBendienst (4) & \\
\hline sonstiges ........ (5) & \\
\hline
\end{tabular}


Denken sle bltte elnmal an die Handelsvertreter, mit denen sie Vertrage abgeschlossen haben. Im Laufe der 2usamenarbeit kunnen dabel sowohl positive als auch weniger positive Eindrucke entstanden seln. Manche Bereiche laufen relbungelos, in anderen sind mbglicherweise Anderungen winschenswert. w1r mbchen von Ihnen wissen, wie sie die gegenwartige zusamnenarbeit mit Ihren Handelsvertretern $1 \mathrm{~m}$ Durchschnitt beurtelien, und ob sie in den genannten Bereichen Anderungen als wilnschenswert ansehen.

Die Buros der Handelsvertreter sind stindig personell besetzt.

Die Buros sind mit allen erforderlichen techniscten Hilfsndtteln arsgestattet (2.B. Telex, Annufbeantworter).

Meine Handelsvertreter verflugen iber eine stindig aktalisierte Kundenkartel.

Die Qualifikation der Mitarbelter in me1nen Handelsvertretungen entspricht den Anforderungen.

D1. Handelsvertreter haben etnen langfristigen Kontakt zu ihren Kunden.

D1e Handelsvertreter erweltarn Inren loundenkreis stundig.

D1e Handelsvertreter wissen, wile sich thr genamter Unsatz auf die verschiedenen Kunder derte1lt. cortiments stiltzen sich die Handelsver treter aff elgene Marktuntersuchungen.

Die Handelsvertrater geben Anregungen fur die intwloklung nover Produkte unseres Unterrehmens.

D1. Handelovertreter beeinfluseen die Co staltung des Sortiments unseres inter nehinens.

Die Prochukte der verschiledenen Hersteller ergunzen sich gut beil unseren Handelsver tretern.

Die Handelsvertreter schlagen uns vor Vertragsabachlub die brancheriblichen Provisionsaltze vor.

Vor der tbernalme elner Vertretung fuhren die Handelevertreter eine Kalkulation ren die Handelovertreter eln

wastziliche Leistumgen (2.B. Auslleferumg, Regalpflege) stellen uns die Handelsvertreter gescandert in pechrung.

Be1 Verchandlungen mit inden haben die Handal overtreter einen Handlungsepielraum tir Preisnachlisea.

Bat Verhandlungan mit kunden können die Handelovertreter die Konditionen (2.B. Rabatte, 2ahlumgsziele) Innerhalb vargegabener Bandbreitan nach elgenem Bxmessen festlegan.

Wir exhalten van den Handelsvertretem in aurreichendem MaBe aktuelle Berichte.

Die Handelevertreter informieren unger Untarnehmen liber litre aigenen Planungen (2.B. Unsatzoilele eines neven Jahres) D1e Handelovertreter b1eten thren Kunden genilgend aktuelle Informationen.

D1e Handelevertroter verwerten unsere Infocmaticnen flir Ihre elgenan Planumgen und Masnatmen.

Die Handelsvertreter ververten die Infor maticinen der kunden fir ihre algenen Planumgen und Masnatmen.

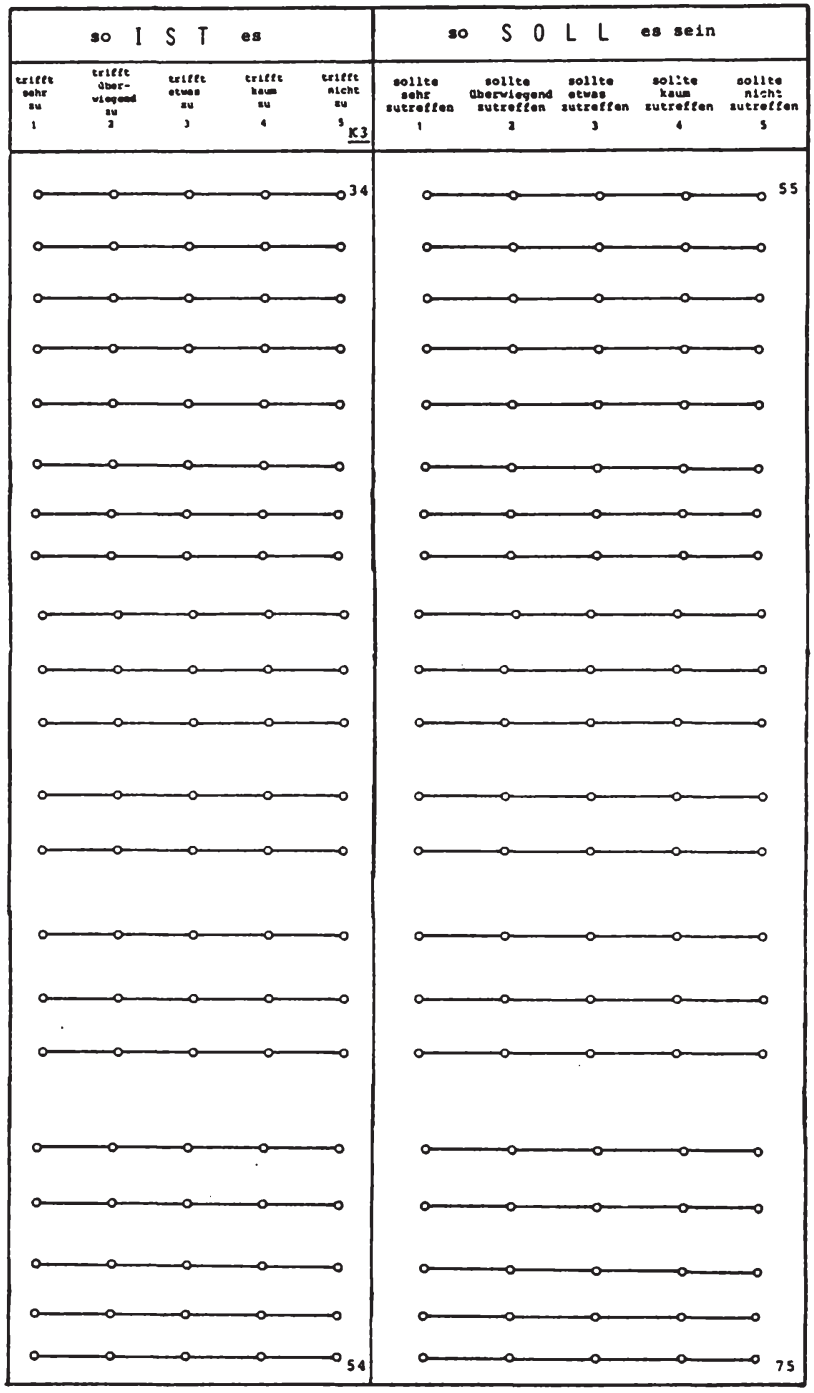


Betrachten sie bitte elnmal Ihr gesamtes Produktangebot. Konzentriert sich Ihre Produktpalette auf eine bestimmte Warengruppe oder verkaufen sie eine Reihe von Waren, die sehr verschleden voneinander sind (wie $z$. B. Wein oder sußwaren/Schrănke oder T1sche)?

(bitte ankreuzen)

Uberwiegend eine warengruppe

mehrer warengruppen

(1)

(2)

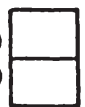

Wieviele solcher Warengruppen bieten Sie insgesamt an?

Anzahl :

Wleviele dieser warengruppen stammen aus ausländischer Produktion (Handelswaren)?

Anzah 1:

Wie hoch war Ihr gesamter Umsatz 1978?

(bitte auf volle Mio.DM runden; ohne MWST)

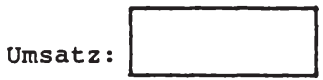

Welche Unsatzanteile entfallen dabei auf importierte bzw. exportierte Produkte (belm Import nur Handelswaren angeben;keine Rohstoffe, d.h. Vorprodukte)?

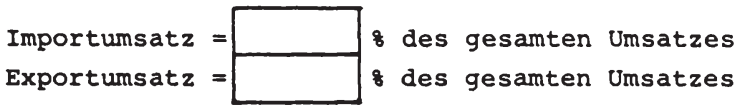

Wer sind Ihre Hauptabnehmergruppen? (bitte ankreuzen)

\begin{tabular}{|lr|r|}
\hline Endverbraucher & (1) & \\
\hline Wiederverkäufer (Händler) & (2) & \\
\hline Weiterverarbelter (Industriebetriebe)(3) & \\
\hline Handwerksbetriebe & (4) & \\
\hline Offentliche Betriebe & (5) & \\
\hline
\end{tabular}

Wievile Beschäfigte hat Ihr Unternehmen insgesamt? Zahl der Beschäftigten:

Wann wurde Ihr Unternehmen gegründet?

Gründungsjahr : 
Welche Rechtsform hat Ihr Unternehmen?

(bitte ankreuzen)

\begin{tabular}{|lr|l|}
\hline Einzelunternehmung & (1) & \\
\hline OHG & $(2)$ & \\
\hline KG & $(3)$ & \\
\hline GmbH & $(4)$ & \\
\hline GmbH \& Co KG & $(5)$ & \\
\hline AG & $(6)$ & \\
\hline
\end{tabular}

In welchem Bundesland ist Ihr Unternehmen angesiedelt?

Bundesland :

Wie groB ist der Ort, in dem Ihr Unternehmen seinen Sitz hat?

\begin{tabular}{|llll|l|}
\hline unter 10.000 & & (1) & \\
\hline 10.000 - unter & 50.000 & $(2)$ & \\
\hline 50.000 - unter & 100.000 & $(3)$ & \\
\hline 100.000 - unter & 250.000 & $(4)$ & \\
\hline 250.000 - unter & 500.000 & $(5)$ & \\
\hline 500.000 - unter 1000.000 & $(6)$ & \\
\hline Uber 1.000 .000 & & $(7)$ & \\
\hline
\end{tabular}

Wieviele Zwelgwerke hat Ihr Unternehmen(Produktionsstätten)?

$($ KeIne $=0)$

Zahl der Zweigwerke:

Wieviele AuBenläger unterhält Ihr Unternehmen?

$($ Keline $=0)$

Zahl der AuBenläger:

Wer unterhält die AuBenläger?

(bitte ankreuzen)

\begin{tabular}{|ll|l|}
\hline Unsere Unternehmung selbst (1) & \\
\hline Speditionsfirmen & $(2)$ & \\
\hline Handelsvertreter & (3) & \\
\hline
\end{tabular}

Wieviele Ihrer Handelsvertreter unterhalten ein eigenes Lager?

Zahl der Handelsvertreterlägèr:

Wieviele Reisende bzw. Handelsvertreter beschäftigt Ihr Unternehmen in der Bundesrepublik?

Zahl der Relsenden

Zahl der Handelsvertreter

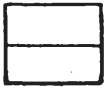

Falls Sie Ihre Erzeugnisse exportieren: Wieviele Reisende bzw. Handelsvertreter sind im Ausland für Ihr Unternehmen tätig?

Zahl der Reisenden im Ausland

$\mathrm{Zahl}$ der Handelsvertreter im Ausland

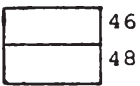


Nach welchen Gesichtspunkten haben Sie Ihren Vertrieb organisiert? (bitte ankreuzen)

Bei den Handelsvertretern

Bei den Reisenden

\begin{tabular}{|c|c|c|c|}
\hline $\begin{array}{l}50 \\
(1)\end{array}$ & $\begin{array}{l}\text { räumlich (z.B. nach } \\
\text { Postleitzonen) }\end{array}$ & h Bundesländern, & $\begin{array}{l}51 \\
\text { (1) }\end{array}$ \\
\hline (2) & $\begin{array}{l}\text { nach Kundengruppen } \\
\text { Warenhäuser) }\end{array}$ & (z.B. Großhandel & $'(2)$ \\
\hline (3) & nach Produktgruppen & & (3) \\
\hline
\end{tabular}

Bitte betrachten Sie einmal die Zusammensetzung Ihres gesamten Umsatzes. Welchen Umsatzantell erzielen Ihre Handelsvertreter, welchen Ihre Reisenden und wie hoch ist der Anteil aus den Geschäften, die mit Kunden direkt abgewickelt wurden? (bitte schätzen Sie Ihre Angaben in Prozent)

$$
\begin{aligned}
& \text { Umsatzanteil der Handelsvertreter } \\
& \text { Umsatzanteil der Reisenden } \\
& \text { Umsatzanteil aus Direktgeschäften }
\end{aligned}
$$

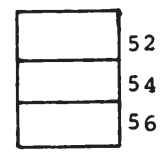

Seit wann arbeitet Ihr Unternehmen mit Handelsvertretern zusammen?

Zahl der Jahre

Angenommen, Sie möchten mit einem neuen bzw. zusätzlichen Handelsvertreter einen Vertrag abschließen, wie nehmen sie den Kontakt zu diesem auf?

\begin{tabular}{|l|l|l|}
\hline Empfehlung bisheriger Handelsvertreter (1) & \\
\hline elgene Anzelgen (Fach-/Tageszeitung) (2) & \\
\hline Erkundigung beim Berufsverband & (3) & \\
\hline Erkundigung/Empfehlung von Kunden & (4) & \\
\hline
\end{tabular}

Welchen Anteil Ihrer gesamten Erzeugnisse vertreiben Sie mit Handelsvertretern bzw. Reisenden? (bitte ankreuzen)

Handel svertreter

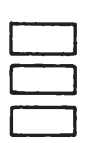
61

(1) alle Produktgruppen die meisten Produktgruppen nur wenige Produktgruppen

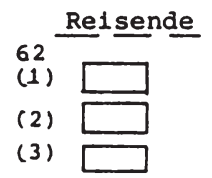

Wenn elner Ihrer Handelsvertreter seine Tätigkeit für Ihr Unternehmen beendet, wodurch werden sie ihn ersetzen?

(1) durch einen neuen Handelsvertreter

(2) durch einen eigenen Reisenden 
Für wieviele verschiedene Unternehmungen arbeiten Ihre Handelsvertreter im Durchschnitt?

Wleviele Kunden betreut jeder Ihrer Handelsvertreter im Durchschnitt?

Wie hoch ist nach Ihrer Meinung der Anteil der Betriebsausgaben Ihrer Handelsvertreter an den Provisionseinnahmen im Durchschnitt?

(bitte in Prozent angeben)

2um Abschlus gestatten sie uns bitte einige Fragen zu Ihrer Person.

wie alt sind sie?

Jahre

Welche Berufsausb1ldung haben Sie?

\begin{tabular}{|c|c|}
\hline kaufm. Lehre & 1) \\
\hline techn. Lehre & 2) \\
\hline Fachhochschule (3) & \\
\hline abgeschlossenes (4) & \\
\hline
\end{tabular}

Welche Stellung haben sie in Ihrem Unternehmen? (bitte ankreuzen)

\begin{tabular}{|c|c|}
\hline Alleinlnhaber & (1) \\
\hline M1t1nhaber & (2) \\
\hline Geschaftsfuhrer & (3) \\
\hline Verkaufsleiter (Innendienst) & (4) \\
\hline Verkaufsleiter (AuBendienst) & (5) \\
\hline
\end{tabular}
w1e lange and sie in Ihrer letzigen Position tat1g? (b1tte in Jahren angeben)

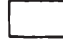

W1. lange sind S1e Inagesamt in Ihram Jetzigen Unternehmen tatig?

w1 oft haben sie mit Ihren Handelsvertretern perounlichen Kontakt (elnschl. Telefon)? 
Fragebogen

' Kunden ' 
WESTF. WILHELMS-UNIVERSITÄT

\section{INSTITUT FUR MARKETING}

DIREKTOR: PROF. DR. H. MEFFERT

\section{F R A G E B O G E N}

In welchem Geschätsbereich (Branche) ist Ihr Unternehmen liberwiegend tätig?

Hauptgeschäftsbereich:

Zu welcher der im folgenden genannten Unternehmensarten zählt Ihr Unternehmen?

(b1tte eine Möglichkeit ankreuzen)

\begin{tabular}{|c|c|}
\hline Industriebetrieb & (1) \\
\hline $\begin{array}{l}\text { Einkaufsverband/ } \\
\text { Genossenschaft }\end{array}$ & (2) \\
\hline Cash und Carry Markt & (3) \\
\hline FachgroBhandel & $(4)$ \\
\hline Discountbetrieb & $(5)$ \\
\hline $\begin{array}{l}\text { Warenhaus oder } \\
\text { anderer Filialbetrieb }\end{array}$ & \\
\hline Facheinzelhandel & $(7)$ \\
\hline
\end{tabular}

Bitte betrachten Sie elnmal Ihren gesamten Elnkauf. In welchem Umfang beziehen Sie Ihre Produkte von den Im folgenden genannten Bezugsquellen? Bitte schätzen Sie Ihre Angaben nach den gesamten Einkaufswerten und notieren die entsprechende Prozentzahl.

\begin{tabular}{|l|c|}
\hline Einkaufsanteil von Reisenden & 8 \\
\hline Einkaufsanteil von Handelsvertretern & 8 \\
\hline Direktbezug von Herstellern & 8 \\
\hline Bezug über eine Einkaufszentrale & 8 \\
\hline
\end{tabular}

Welche Telle Ihres Sortiments kaufen Sie von Handelsvertretern, welche von Reisenden? (bitte ankreuzen)

von Handelsvertretern

$18 \square$

(1) gesamtes Sortiment von Rei senden

(2) wesentliche Teile
$\square$ (3) ergänzende Teile


Denken Sle bitte einmal an die Handelsvertreter, von denen Sie Waren beziehen. Im Laufe der zusamenarbeit können dabei sowohl positive als auch weniger positive Eindrucke entstanden sein. Manche Bereiche laufen reibungslos, in anderen sind moglicherwelse Xinderungen walnschenswert.

Wir mochten von Ihnen wissen, wie sie die gegenwartige zusammenarbelt mit den Handelsvertretern $1 m$ Durchschnitt beurtellen, und ob Sie in den genannten Bereichen Anderungen als wünschenswert ansehen.

Die Büns der Handelsvertreter sind ständig personell besetzt.

Die Buros sind utt ailen erforderlichen technischen Hilfsnitteln ausgestattet (2.B. Telex, Anrufbeantworter).

Die Qualifikation der Mitarbelter in den Handelsvertretungen entspricht den Anforderungen.

Die Handelsvertreter haben einen langfristigen Kontakt zu Ihren Kunden.

Bel der Zusamensetzung inres Vertretungssortiments stiltzen sich die Handelsvertreter auf elgene Marktuntersuchungen.

Die Hancielsvertreter geben Anregungen fur die Entwicklung neuer Produkte der Hersteller.

Die Handelsvertreter beeinflussen die Sortimentsgestaltung der Hersteller.

Die Produkte der verschiledenen Hersteller ergänzen sich gut bei den Handelsvertretern.

Die Handelsvertreter haben einen Handlungssplelraum für Preisnachlässe.

Die Handelsvertreter kơnnen die Kanditionen (z.B. Rabatte, zahlungsziele) innerhalb vorgegebener Bandbrel ten nach elgenem Eromes sen festlegen.

Die Handelsvertreter bieten uns genilgend aktuelle Inforiationen.

Die Handelsvertreter verwerten unsere Informationen für thre eigenen Plamungen und Masnarmen.

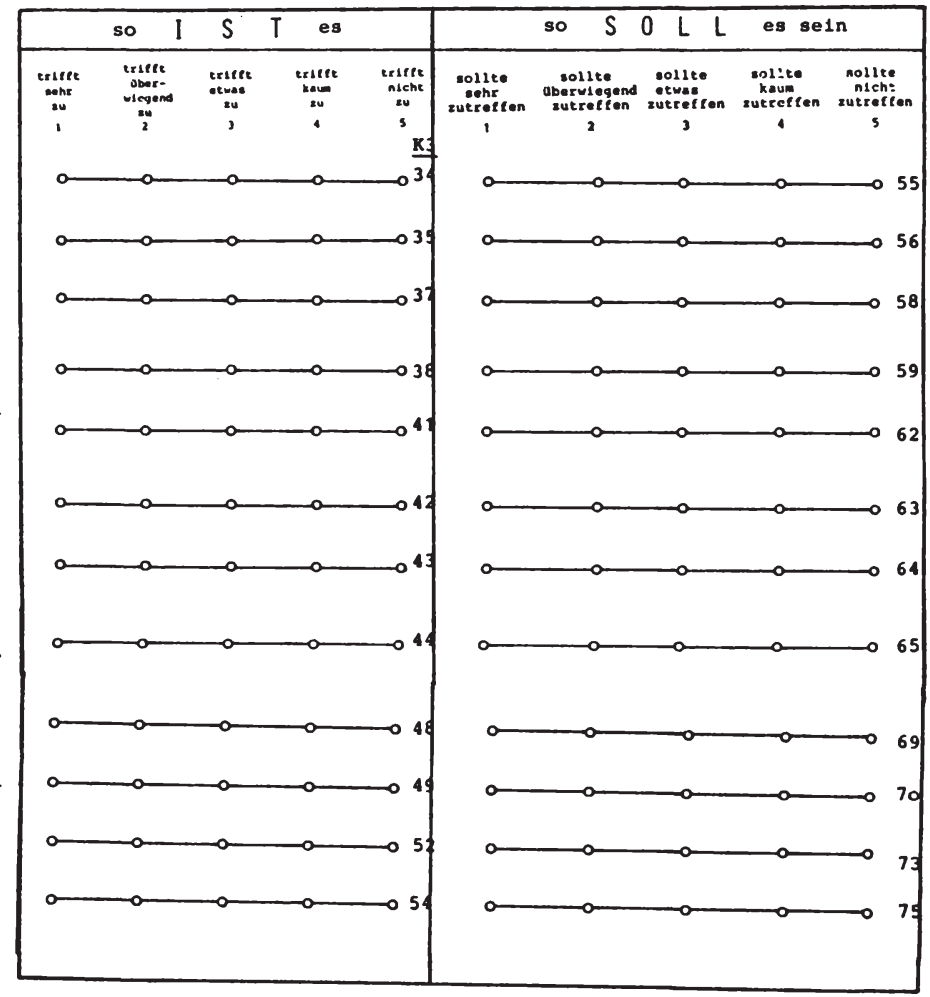


Gestatten sie uns bitte abschließend noch einige Fragen zu Ihrem Unternehmen. Die Angaben werden - wie alle ubrigen Antworten - selbstverständlich vertraulich behandelt.

Betrachten ste bitte einmal Ihr gesamtes produktangebot. Konzentriert sich Ihre Produktpalette auf eine bestimmte Warengruppe oder verkaufen sie eine Relhe von Waren, die sehr verschieden voneinander sind (z.B. Wein oder süBwaren, Schrănke oder Tische)?

(bitte ankreuzen)

überwiegend eine warengruppe mehrere warengruppen

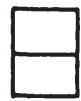

Wieviele solcher Warengruppen bieten Sie insgesamt an?

Zahl der Warengruppen:

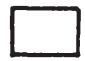

Wie hoch war Ihr gesamter Umsatz 1978?

(In DM ohne MWST)

Umsatz:

Wer sind Ihre Hauptabnehmergruppen?

(bitte ankreuzen)

\begin{tabular}{|lr|r|}
\hline Endverbraucher & (1) & \\
\hline Wiederverkăufer (Hăndler) & (2) & \\
\hline Weiterverarbeiter (Industriebetriebe)(3) & \\
\hline Handwerksbetriebe & (4) & \\
\hline Öffentliche Betriebe & (5) & \\
\hline
\end{tabular}

Wenn Sie Großhändler sind: Wie groß 1st Ihre Lagerfläche?

$$
\text { Lagerfläche: }
$$

Wenn Sie Elnzelhändler sind: Wie groB ist Ihre Verkaufsfläche?

$$
\text { Verkaufsfläche: }
$$

Wenn Sie in einem Unternehmen mit mehreren Geschäftsstellen arbeiten, wo sind sie beschaftigt? (bitte ankreuzen)

$$
\begin{aligned}
& \text { in einer der Niederlassungen/Filialen } \\
& \text { in der Zentrale/Hauptverwaltung }
\end{aligned}
$$

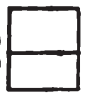

wieviele Filialen hat Ihr Unternehmen insgesamt? 
Wleviele Beschäfigte hat Ihr Unternehmen Insgesamt?

Zahl der Beschaftigten:

Wann wurde Ihr Unternehmen gegründet?

Gründungsjahr:

Welche Rechtsform hat Ihr Unternehmen?

\begin{tabular}{|l|l|l|}
\hline EInzelunternehmung $(1)$ & \\
\hline OHG & $(2)$ & \\
\hline KG & $(3)$ & \\
\hline GmbH & $(4)$ & \\
\hline GIIBH \& CO KG & $(5)$ & \\
\hline AG & $(6)$ & \\
\hline
\end{tabular}

In welchem Bundesland ist Ihr Unternehmen angesiedelt (Stammsitz)? Bundesland:

Wie weit erstreckt sich Ihr Angebot? (bitte ankreuzen)

reglonal begrenzt (1) bundeswe1t (2)

Wie groB ist der Ort, in dem Ihr Unternehmen seinen Sitz hat?

\begin{tabular}{|llll|l|}
\hline unter 10.000 & & $(1)$ & \\
\hline 10.000 - unter & 50.000 & $(2)$ & \\
\hline 50.000 - unter & 100.000 & $(3)$ & \\
\hline 100.000 - unter & 250.000 & $(4)$ & \\
\hline 250.000 - unter & 500.000 & $(5)$ & \\
\hline 500.000 - unter & 1.000 .000 & $(6)$ & \\
\hline über 1.000 .000 & & $(7)$ & \\
\hline
\end{tabular}

Nach welchen Gesichtspunkten haben Sie Ihren Einkauf organisiert? (bitte ankreuzen)

(1) nach Bezugsquellen (Lieferanten)

(2) nach eigenen Sortimentsgruppen

nach speziellen Produktmerkmalen

(3) (z.B. Neuheitsgrad, Erklärungsbedurftigkeit) 
Wieviele Personen in Ihrem Unternehmen sind mit dem Einkauf beschäftigt?

Zahl der Elnkăufer:

Welche anderen Aufgaben (auBer dem Elnkauf und damit verbundene Tätigkelten) haben diese Personen?

Wer entscheidet uber den endgulticien Kauf einer Ware?

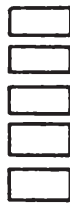

(1)Einkäufor allein

(2)Einkaufsleiter allein

(3)Inhaber (Geschaftsführer) allein

(4)mehrere Personen gemeinsam

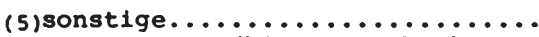

(bitte angeben)

Für wieviele verschiedene Hersteller arbeiten die Handelsvertreter im Durchschnitt, die Ihr Unternehmen besuchen?

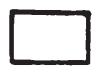

Betrachten Sie bitte elnmal Ihre gesamte Autragsabwicklung mit den Handelsvertretern. Wie groB ist der Antell der Aufträge, die Sie über Telefon (und Telex) mit den Handelsvertretern abwickeln? (evtl. Schätzung)

Auftragsabwicklung über Telefon/Telex: $\%$

Wie hoch 1st nach Ihrer Melnung der Anteil der Betrlebsausgaben eines Handelsvertreters an seinen Provisionseinnahmen im Durchschnitt? (bitte in Prozent angeben)

Zum AbschluB gestatten Sie uns bitte einige Fragen zu Ihrer Person. sie sind

mănnlich weiblich 
wie alt sind sie?

Welche Berufsausbildung haben sie

\begin{tabular}{|ll|l|}
\hline kaufm. Lehre & (1) & \\
\hline techn. Lehre & (2) & \\
\hline Fachhochschule & (3) & \\
(z.B. Ing.grad.) & & \\
\hline $\begin{array}{ll}\text { abgeschbssenes } \\
\text { Universitătsstudium }\end{array}$ & \\
\hline
\end{tabular}

Welche Stellung haben Sie in Ihrem Unternehmen? (bitte ankreuzen)

\begin{tabular}{|ll|l|}
\hline Alleininhaber & (1) & \\
\hline Mitinhaber & (2) & \\
\hline Geschäftsfuhrer & (3) & \\
\hline Elnkaufsleiter & (4) & \\
\hline Facheinkăufer & (5) & \\
\hline
\end{tabular}

wie lange sind sie in Ihrer jetzigen Position tătig?

(bitte in Jahren angeben)

Jahre

Wie lange sind sie Insgesamt in Ihrem jetzigen Unternehmen tätig?

Wie oft haben sie mit Handelsvertretern persónlichen Kontakt (einschl. Telefon)?

$$
\text { Anzahl der Kontakte pro Monat: }
$$

Welcher Anteil Ihrer Arbeitszeit entfallt auf die zusammenarbeit mit den Handelsvertretern?

(bitte in Prozent schatzen) 


\section{Literaturverzeichnis}

Ahlert, D.,

Althans, J.

Andritzky, $\mathrm{K}$.

Bachmann, R.

Bachmann, W.

Bales, R.F.

Banton, M.

Batzer, E., Meyerhöfer, w.

Batzer, E., Meyerhöfer, W.

Baumann, J.K.
Grundzüge des Marketing, Düsseldorf 1980

Standardisierung von Marketingkonzeptionen in internationalen Unternehmen aus der Sicht von Tochtergesellschaften - Eine explorative Studie auf einem überseeischen Markt, Arbeitspapiere des Instituts für Marketing der Universität Münster, Hrsg.: H. Meffert, Nr. 20, Münster 1980

Die Operationalisierbarkeit von Theorien zum Konsumentenverhalten, Berlin 1976

Das neue Recht des Handelsvertreters, Kissing 1978

Welche Information braucht der Fabrikant von den Handelsvertretern?, in: HV + HM 1963, S. $864 \mathrm{ff}$.

Task roles and social roles in problem solving groups, in: Current studies in social psychology, Hrsg.: I.D. Steiner, M. Fishbein, New York 1965 , s. $321 \mathrm{ff}$.

Roles - An Introduction to the Study of Social Relations, London 1965

Der Handelsvertreter - individueller Absatzmittler im modernen Markt, in: $\mathrm{HV}+\mathrm{HM}$ 1969, S. $241 \mathrm{ff}$.

Aufgaben, Stellung und Entwicklungsperspektiven der Handelsvertretung in der Bundesrepublik Deutschland, Berlin 1970

Der Handelsvertreter - eine absatzpolitische Entscheidungsalternative, Diss. Wien 1966 
Behling, 0. ,

Schriesheim, C.

Beier, F.J.,

Stern, L.W.

Belonax, J.A., Mittelstaedt, R.A.

Berekoven, L.,

Eckert, W.,

Ellenrieder, P.

Bethge, K.H.

Beutel, P. u.a.

Biddle, B.J.

Biddle, B.J.

Biddle, B.J.,

Thomas, E.J. (Hrsg.)

Bless, H.J.
Organizational Behavior, Boston 1976

Power in the Channel of Distribution, in: Distribution Channels: Behavioral Dimensions, Hrsg.: L.W. Stern, Boston 1969, S. 92 ff.

Evoked set size as a function of number of choice criteria and information variability, in: Advances in Consumer Research, Hrsg.: H.K. Hunt, Provo 1978, S. $48 \mathrm{ff}$.

Marktforschung. Methodische Grundlagen und praktische Anwendung, Wiesbaden 1977

Mit Handelsvertretern erfolgreich verkaufen, in: MA 1973, Heft 2, S. $67 \mathrm{ff}$.

SPSS - Statistik Programmsystem für die Sozialwissenschaften, 2. Aufl., Stuttgart 1978

The Present Status of Role Theory, Columbia 1961

Roles, Goals and Value Structures in Organizations, in: New Perspectives in Organization Research, Hrsg.: W.W. Cooper, New York usw. 1964, S. $150 \mathrm{ff}$.

Role Theory, Concepts and Research, New York usw. 1966

Wieviel Freiraum hat der freie Handelsvertreter, in: VuM 1974, Heft 12, S. $3 \mathrm{ff}$. 
Bless, H.J.

Bleymuiller, J., Gehlert, $G$.

Bleymüller, J., Gülicher, H. , Gehlert, G.

Bobrow, E.E.

Böhler, H.

Briechle, J.

Bruhn, M.

Claessens, D.

Cohen, J.B. (Hrsg.)

Cohen, R., Richmond, S., Stock, J.S., Semon, T.T.

Collins, R.H.

Czaia, U.
Unwirtschaftlichkeit wider Willen, in: HV + HM 1976, S. $451 \mathrm{f}$.

Statistik für Wirtschaftswissenschaften, in: Wist 1977, S. $23 \mathrm{ff}$.

Statistik für Wirtschaftswissenschaften, in: Wist 1978, S. 369 ff.

Marketing through Manufacturers' Agents, New York 1976

Methoden und Modelle der Marktsegmentierung, Stuttgart 1977

Ưberprüfung des Vertretungssortiments, in: HV + HM 1965, S. $1058 \mathrm{f}$.

Das soziale Bewußtsein von Konsumenten, Wiesbaden 1978

Rolle und Macht, 2. Aufl., München 1970

Behavioral Science Foundations of Consumer Behavior, New York usw. 1972

Sampling in Marketing Research, in: AMA 'Marketing Research Techniques' Series No. 3, 0.0. 1970

Manufacturer's Representatives for Small Firms, in: JoSBM 1978, S. $13 \mathrm{ff}$.

Schriftliche Befragung, in: IUA 1978, S. $174 \mathrm{ff}$. 
Dahlhoff, H.D.

Dahrendorf, R.

Dahrendorf, R.

Dichtl., E.,

Bauer, H.H.

Dichtl., E.,

Raffée, H.,

Niedetzky, H.M.,

Engel, J.F.,

Kollat, D.T.,

Blackwell, K.D.

Evan, W.M.

Falk, B.

Finkenrath, R.

Forschungsverband für den Handelsvertreter- und Handelsmaklerberuf (Hrsg.), CDH-Statistik 1978, Köln 1979 (unveröffentlicht)
Individuelle Wertorientierungen Analyse und Aussagewert personenspezifischer Werthierarchien im Marketing, Arbeitspapiere des Instituts für Marketing der Universität Münster, Hrsg.: H. Meffert, Nr. 23, Münster 1980

Homo Sociologicus, 13. Aufl., Opladen 1974

Rolle und Rollentheorie, in: Wörterbuch der Soziologie, Hrsg.: W. Bernsdorf, Stuttgart 1969, S. 902 ff.

Die Hersteller - Handels- Kommunikation bei neuen Produkten auf dem Prüfstand, Nr. 6 der Information zur Rationalisierung der Kommunikation, Frankfurt 1978

Reisende oder Handelsvertreter - Eine . Anleitung zur Lösung eines Entscheidungsproblems mit praktischen Vorschlägen, München 1981

Consumer Behavior, Hinsdale 1978

Toward a Theory of Inter-Organizational Relations, in: MS 1965, S. $217 \mathrm{ff}$.

Zur Bedeutung des DienstleistungsMarketing, in: Dienstleistungsmarketing, Hrsg.: B. Falk, München 1980, S. $10 \mathrm{ff}$.

Mehr Gewinn durch gewinnorientierte Entlohnung im Vertrieb, München 1978 
Frank, R.E., Green, P.E.

Frank, E.R., Massy, W.F., Wind, $Y$.

$$
\text { Gill, C.E., }
$$
Stern, L.W.

Goode, W.J.

Grabner, J.R., Rosenberg, L.J.

Grace, D. ,

Pointon, T.

Green, P.E.

Gröne, A.

Grochla, E., Welge, M.K.

Gross, H.
Numerical Taxonomy in Marketing Analysis: A Review Article, in: D.A. Aaker (Hrsg.), Multivariate Analysis in Marketing: Theory \& Application, Belmont 1971, S. $300 \mathrm{ff}$.

Market Segmentation, Englewood Cliffs 1972

Roles and Role Theory in Distribution Channel Systems, in: Distribution Channels: Behavioral Dimensions, Hrsg.: L.W. Stern, New York usw. 1969, S. $22 \mathrm{ff}$.

A Theory of Role Strain, in: Behavioral Science Foundations of Consumer Behavior, Hrsg.: J.B. Cohen, New York usw. 1972, S. $407 \mathrm{ff}$.

Communication in Distribution Channel Systems, in: Distribution Channels: Behavioral Dimensions, Hrsg.: L. W. Stern, Boston 1969, S. $227 \mathrm{ff}$.

Marketing Research Through the Salesforce, in: IMM 1980, S. $53 \mathrm{ff}$.

Analyzing Multivariate Data, Hinsdale 1978

Marktsegmentierung bei Investitionsgüter Wiesbaden 1977

Einfuhrung in die verhaltenstheoretisch orientierten Ansätze, in: Organisationstheorie, Hrsg.: E. Grochla, 1. Teilband, Stuttgart 1975, S. $90 \mathrm{ff}$.

Dienstleistung - Chance des Handelsvertreters, in: HV + HM 1969, S. 580 ff. 
Gross, H.

Gross, N. , Mason, S.W., McEachern, W.A.

Gülicher, H., Gehlert, G.

Gutenberg, E.

Hafermalz, 0 . Hartan, M.

Harvey, D.F., Brown, D.R.

Herbert, T.T.

Hirsig, B.W.

Höfner, $K$.
Der Handelsvertreter im Wandel der Märkte, Düsseldorf - Wien 1966

Explorations in Role Analysis, New York 1958

Statistik für Wirtschaftswissenschaftler, in: Wist 1978, S. $420 \mathrm{ff}$.

Grundlagen der Betriebswirtschaftslehre, zweiter Band, Der Absatz, 15. Aufl., Berlin-Heidelberg-New York 1976

Schriftliche Befragung - Möglichkeiten und Grenzen, Wiesbaden 1976

Marketing- und Vertriebsorganisation in mittelständischen Unternehmen, Teil 1, Eingliederung des Marketing in die Aufbauorganisation der befragten Unternehmen, RKW-Schriftenreihe marktorientierte Unternehmensführung Absatzwirtschaft, Frankfurt 1974

An Experimental Approach to Organization Development, Englewood Cliffs 1976

Dimensions of Organizational Behavior, New York-London 1976

Agents show their effectiveness in the Automative Aftermarket, in: ASM 1979, Heft 10, S. 31

Leitfaden für das praktische Marketing in mittleren und kleinen Unternehmen der Investitions- und Gebrauchsgüterindustrie, Frankfurt 1978 
Hoffmann, F.

Hüttner, $M$.

Hughes, D.G.,

Ray, M.L. (Hrsg.)

Hull, C.M.,

Nie, N.H.

Hunsinger, $\mathrm{H}$.

Irgel, L.

Jeske, J.J.

Kahn, R.L. u.a.

Kamitz, R.

Kast, F.E.,

Rosenzweig, J.E.

Katz, D. ,

Kahn, R.L.

Kemna, M.
Entwicklung der Organisationsforschung, Wiesbaden 1973

Informationen für Marketing-Entscheidungen, München 1979

Buyer/Consumer Information Processing, Chapel Hill 1974

SPSS Update, New York usw. 1979

Durch bessere Information erfolgreicher an den Handel verkaufen, Nr. 7 der Information zur Rationalisierung der Kommunikation, Frankfurt 1979

Wieder im Kommen: der Handelsvertreter, in: MJ 1976, S. $323 \mathrm{ff}$.

Der Musterkoffer reicht schon längst nicht mehr, in: FAZ NN. 281 vom 3.12 .1977 , S. 11

Organizational Stress: Studies in Role Conflict and Ambiguity, New York usw. 1964

Wissenschaftslogische Probleme beim Aufbau einer Theorie der Konflikte, in: Unternehmensbezogene Konfliktforschung, Hrsg.: G. Dlugos, Stuttgart $1979, \mathrm{~S} .33 \mathrm{ff}$.

Organization and Management - A

Systems Approach, New York usw. 1970

The Social Psychology of Organizations, New York 1966

Key Account Management, München 1979 
Kim, J.O.

Kim, J.G. ,

Kohout, F.J.

Kim, J.O.,

Kohout, F.J.

Kimmeskamp, G.
Factor Analysis, in: SPSS, Hrsg.: N.H. Nie u.a. 2. Aufl., New York usw. 1975, S. $468 \mathrm{ff}$.

Special Topics in Linear Models, in: SPSS, Hrsg. N.H. Nie U.a., 2. Aufl., New York usw. 1975, S. 373 ff.

Multiple Regression Analysis:

Subprogram Regression, in: SPSS,

Hrsg. N.H. Nie u.a., 2, Aufl., New

York usw. 1975, S. $320 \mathrm{ff}$.

Tätigkeitsbereiche und Dienstleistungsangebot von Handelsvertretungen Ergebnisse einer empirischen Untersuchung, Arbeitspapiere des Instituts für Marketing der Universität Münster, Mrsg.: H. Meffert, Nr. 18, Münster 1979

Kimmeskamp, G.

Die Handelsvertretung im Meinungsbild von Industrie und Handel, in: FfH-Mitteilungen, Heft 11, 1980

Entscheidungsprozesse, 1. Band, Wiesbaden 1970

Entscheidungsprozesse, 3. Band, Wiesbaden 1971

Marketing-Praxis mit Handelsvertretern, Gernsbach 1973

Handelsvertreter und Marketing, in: Marketing-Enzyklopädie, Band 1, München 1974, S. $931 \mathrm{ff}$.

Marketing mit Handelsvertretern im härteren Markt, in: HV + HM 1976, S. $191 \mathrm{ff}$. 
Kook, W.

Kopp, M.

Kotler, P.

Krautter, J.

Kroeber-Riel, W.

Krüger, W.

Kuzela, L.

Lampe, A.

Lebensmittel-Zeitung (Hrsg.), Der Markenartikel-Reisende, o.0. 1978

Leicher, R.

Linton, $\mathrm{R}$.
Einstellungen zur Universität - Operationalisierung und empirische Prüfung verhaltenswissenschaftlicher Hypothesen am Beispiel der Stadtbevölkerung von Münster, Frankfurt 1981

Hypothesenformulierung in der $\mathrm{Ab}-$ satzforschung, Betriebswirtschaftliche Schriften, Heft 20, Berlin 1972

Marketing Management - Analyse, Planung und Kontrolle, Wiesbaden 1974

Zum Problem der optimalen Marktsegmentierung, in: ZfB 1975, S. $109 \mathrm{ff}$.

Konsumentenverhalten, 2. Aufl., München 1980

Zur methodischen und inhaltlichen Problematik der Analyse von Zielbildungsprozessen, in: Unternehmensbezogene Konfliktforschung, Hrsg.: G. Dlugos, Stuttgart 1979, S. $277 \mathrm{ff}$.

How to work with a Manufacturer's Rep, in: Industry Week 1977, Heft 2, S. $40 \mathrm{ff}$.

Stellung und Aufgabe des Handelsvertreters in der Gesamtwirtschaft, aus dem Nachlaß herausgegeben von J. Tiburtius, Berlin 1962
Telefon-Lektion für Handelsvertreter, in: VuM, Heft 10, 1980, S. $64 \mathrm{ff}$.

The Study of Man, New York 1936 
Linton, R.

Levinson, D.J.

Luhmann, $\mathrm{N}$.

Maas, R.M.

March, J.G., Simon, H.A.

Mayntz, R.

Meffert, $H$.

Meffert, $H$.

Meffert, $H$.

Meffert, $H$.
The Cultural Background of Personality, London 1945

Role, Personality and Social structure in the organizational setting, in: Sociology, Hrsg.: S.M. Lipset, N.J. Smelser, Englewood Cliffs 1961, S. $299 \mathrm{ff}$.

Funktionen und Folgen formaler Organisation, Berlin 1964

Absatzwege - Konzeptionen und Modelle, Wiesbaden 1980

Organisation und Individuum, Wiesbaden 1976

Soziologie der Organisation, Reinbek 1963

Die Leistungsfähigkeit der entscheidungs- und systemorientierten Marketing-Theorie, in: Wissenschaftsprogramm und Ausbildungsziele der Betriebswirtschaftslehre, Tagungsberichte des Verbandes der Hochschullehrer für Betriebswirtschaft e.V. , Hrsg.: G.v. Kortzfleisch, Berlin 1971

Modelle des Käuferverhaltens und ihr Aussagewert für das Marketing, in: ZfgSt 1971, S. $326 \mathrm{ff}$.

Absatztheorie, systemorientierte, in: HdA, Hrsg.: B. Tietz, Stuttgart 1974, Sp. $138 \mathrm{ff}$.

Produktivgüter - Marketingforschung im System des Marketing, in: Der Markt 1974, S. $6 \mathrm{ff}$. 
Meffert, $\mathrm{H}$.

Meffert, H. (Hrsg.)

Meffert, $H$.

Meffert, $\mathrm{H}$.

Meffert, $\mathrm{H}$.

Meffert, $\mathrm{H}$.

Meffert, $\mathrm{H}$.

Meffert, $H$.

Meffert, $\mathrm{H}$.

Meffert, $H$.
Vertikales Marketing und Marketingtheorie, in: H. Steffenhagen, Konflikt und Kooperation in Absatzkanälen, Wiesbaden 1975, S. 15 ff.

Marketing heute und morgen. Entwicklungstendenzen des Marketings in Theorie und Praxis, Wiesbaden 1975

Die Handelsvertretung im Spannungsfeld des Marketing-systems, in: HV + HM 1976, S. 83 ff. und $135 \mathrm{ff}$.

Die Handelsvertretung in den $80 e r$ Jahren, in: HV + HM 1977, S. $786 \mathrm{ff}$.

Die Information ist verbesserungsfähig, in: HV + HM 1979, S. $1436 \mathrm{ff}$.

Die Einführung des Kundenmanagements als Problem des geplanten organisatorischen Wandels, Arbeitspapiere des Instituts für Marketing der Universität Münster, Nr. 16, Münster 1979

Perspektiven des Marketing in den 8oer Jahren, in: DBW 1980, S. 59 ff.

Perspektiven des Marketing in den 8oer Jahren - ein Uberblick des Herausgebers, in: Marketing im Wandel, Hrsg.: H. Meffert, Wiesbaden 1980, S. $3 \mathrm{ff}$.

Marketing. Einführung in die Absatzpolitik, 5. Aufl., Wiesbaden 1980

Verhaltenswissenschaftliche Aspekte vertraglicher Vertriebssysteme, in: Vertragliche Vertriebssysteme zwischen Industrie und Handel, Hrsg.:

D. Ahlert, Wiesbaden 1981, S. $99 \mathrm{ff}$. 
Meffert, H. , Kimmeskamp G.

Meffert, H., Steffenhagen, $\mathrm{H}$.

Meffert, H., Steffenhagen, H. , Freter, H. (Hrsg.)

Merton, R.K.

Merton, R.K.

Merton, R.K.

Meyer, P.W.,

Tostmann, T.

Meyerhöfer, W.

Mills, T.M.

Newton, D.J.
Die Handelsvertretung im Meinungsbild von Industrie und Handel Eine empirische Untersuchung unter Berücksichtigung verschiedener Branchen, Arbeitspapiere des Instituts für Marketing der Universität Münster, Hrsg.: H. Meffert, Nr. 22, Münster 1980

Konflikte zwischen Industrie und Handel - Empirische Untersuchungen im Lebensmittelsektor der BRD, Wiesbaden 1976

Konsumentenverhalten und Information, Wiesbaden 1979

Social Theory and Social Structure, Glencoe 1957

The Role-set: Problems in Sociological Theory, in: BJS 1957, S. $110 \mathrm{ff}$.

Instability and Articulation in the Role-Set, in: Role Theory: Concepts and Research, Hrsg.: B.J. Biddle, E.J. Thomas, New York usw. 1966, S. $282 \mathrm{ff}$.

Dienstleistungsmarketing, in: JAVF 1978 , S. 286 ff.

Die Handelsvermittlung - wichtiges Bindeglied in der Wirtschaft, in: ifo-Schnelldienst vom 14.5.1976, S. $23 \mathrm{ff}$.

Soziologie der Gruppe, München 1974

Role of Premium Rep more than a Salesman, in: Advertising Age, Nr. 42 v. 18. Okt. 1978, s. $18 \mathrm{f}$. 
Nie, N.H. u.a.

Oeser, O.A., Harary, F.

o.V.

o.V.

o.v.

O.V.

o.V.

Parsons, T.

Parsons, T., Shils, E.A.

Pohl, F.

Popitz, H.

Porter, L.W., Lawler, E.E.
SPSS - Statistical Package for the Social Sciences, 2. Aufl., New York usw. 1975

A mathematical model for structure role theory, in: Human relations 1962, S. $89 \mathrm{ff}$.

Handelsvertreter setzen viel um, in: Handelsblatt $\mathrm{Nr} .243$ vom 19.12.77, S. 11

Verkaufsleiter: Resultat einer Umfrage, in: VuM, Heft $3,1978, \mathrm{~S}$. $44 \mathrm{ff}$.

Ein neues Berufsbild angestrebt, in: Handelsblatt $\mathrm{Nr} .114$ vom 10.7.1978, Nr. 19

Welche Auswirkungen haben Kooperation und Konzentration, in: Lz Nr. 41, vom 13.10.1978, S. 8

Konflikte erkennen und vermeiden lernen, in: LZ $\mathrm{Nr} .9$ vom 2.3.1979, S. 104

The Social System, Glencoe 1964

The Content of Roles, in: Role Theory, Concepts and Research, Hrsg.: Biddle, B.J., Thomas, E.J., New York usw. 1966, S. $239 \mathrm{ff}$.

Marktsegmentierung mit multivariaten Verfahren, Diss. Münster 1977

Der Begriff der sozialen Rolle als Element der soziologischen Theorie, Tübingen 1967

Managerial Attitudes and Performance, Homewood 1968 
Quitzau, M.

Rathme11, J.M.

Ridgeway, V.

RKW (Hrsg.)

$\mathrm{RKW}$ (Hrsg.)

Rohlmann, P.

Rommetveit, R.R.

Roscoe, A.M. ,

Lang, D.,

Sheth, J.N.

Rosenbloom, B.

Rosenstiel, L.v.
Wer kann's besser, in: Lz Nr. 47 vom 24.11 .1978 , S. 86

Marketing in the Service Sector, Cambridge Mass. 1974

Administration of Manufacturer-DealerSystems, in: ASQ 1957, S. $464 \mathrm{ff}$.; wiederabgedruckt in: Distribution Channels: Behavioral Dimensions, Hrsg.: L.W. Stern, Boston 1969, S. $117 \mathrm{ff}$.

Marketing in mittleren und kleinen Industrieunternehmen dargestellt am Beispiel der Bekleidungsindustrie, Frankfurt 1976

Marketing in mittleren und kleinen Industrieunternehmen dargestellt am Beispiel der Wohnmöbel-Industrie, Frankfurt 1979

Marketing in der Rezession. Ein Beitrag zur antizyklischen Produktund Programmolitik bei langlebigen Konsumgütern, Wiesbaden 1977

Social Norms and Roles, Explorations in the Psychology of Enduring Social Pressures, Oslo-Minneapolis 1955

Follow-up Methods, Questionaire Lenth and Market Differences in Mail Surveys, in: JoM 1975, S. 20 ff.

Marketing Channels. A Management View, Hinsdale 1978

Grundlagen der Organisationspsychologie, Stuttgart 1980 
Sader, M.

Sarbin, T.R., Allen, V.L.

Sarbin, T.R., Turner, R.H.

Sherif, C.W.

Sibley, S.D., Teas, R.K.

Siekamp, W.

Sims, J.T., Foster, J.R., Woodside, A.G.

Schenk, H.G., Spannagel, R., Wölk, A.

Schildmann, G.

Schmidt-Bräkling, R. Zielgruppenbestimmung mit Hilfe von

Schröder, H. Einstellungsskalen - Ein Beitrag zur Marktsegmentierung, Diss. Münster 1973

Rollentheorie, in: Handbuch der Psychologie, 1. Halbband, Göttingen 1975

Role-Theory, in: The Handbook of Social Psychology, Hrsg.: G. Lindzey, E. Aronson, Reading 1968, S. $488 \mathrm{ff}$.

Role, in: International Encyclopedia of the Social Sciences, Hrsg.:

D.L. Sills, Vol. 13, New York 1972, S. 546 ff.

Orientation in Social Psychology, New York usw. 1976

The Manufacturer's Agent in Industrial Distributions, in: IMM 1979, S. $286 \mathrm{ff}$.

Die Leistung des Handelsvertreters in der modernen Marktwirtschaft, in: asw 1953, Heft 6, S. $4 \mathrm{ff}$.

Marketing Channels - Systems and Strategies, New York usw. 1977

Funktionen und Leistungen der Handelsvertretung im Wettbewerb der Vertriebssysteme, Berlin 1974

Der Handelsvertreter in der Marketing-Konzeption, in: HV + HM 1969, S. $302 \mathrm{ff}$.

Der Handelsvertreter im Marketing, Handelsvertreter-Praxis Heft 10, Hrsg.: Forschungsverband für den Handelsvertreter- und Handelsmaklerberuf, Braunschweig 1979 


\begin{abstract}
Statistisches Bundesamt (Hrsg.), Fachserie F, Groß- und Einzelhandel-Gastgewerbe-Fremdenverkehr, Handels- und Gaststättenzählung 1968, III. Handelsvermittlung, Stuttgart-Mainz 1968
\end{abstract}

Steffenhagen, $\mathrm{H}$.

Vertikales Marketing, in: MarketingEnzyklopädie, Band 2, Mưnchen 1974, S. $675 \mathrm{ff}$.

Steffenhagen, $\mathrm{H}$.

Steinhausen, D.

Steinhausen, D., Langer, $\mathrm{K}$.

Stern, L.W., Brown, J.W.

Stern, L.W., El-Ansary, A.I.

Stern, I.W., Heskett, J.L.

Stewart, D.W.,

Sturm, M., Vajna, T.

Tenbruck, F.H.
Konflikt und Kooperation in Absatzkanälen, Wiesbaden 1975

Neue Prozedur Cluster im SPSS -8 als lokale Erweiterung, in: inforum 1980, Heft 2, S. $27 \mathrm{ff}$.

Clusteranalyse, Berlin-New York 1977

Distribution Channels: A Social Systems Approach, in: Distribution Channels: Behavioral Dimensions, Hrsg.: L.W. Stern, Boston 1969, S. $6 \mathrm{ff}$.

Marketing Channels, Englewood Cliffs 1977

Conflict Management in Interorganization Relations, in: Distribution Channels: Behavioral Dimensions, Hrsg.: L.W. Stern, Boston 1969, S. $288 \mathrm{ff}$.

The Application and Misapplication of Factor Analysis in Marketing Research, in: JoMR 1981 , s. 51 ff.

Grundzüge des Faktorenanalyse, in: Techniken der empirischen Sozialforschung, Hrsg.: J.v. Koolwijk, M. Wieken-Mayser, Bd. 5, Testen und Messen, München 1976

Zur deutschen Rezeption der Rollentheorie, in: KzfSS 1961, S. $1 \mathrm{ff}$. 
Thompson, J.D.

Thomas, E.J., Biddle, B.J.

Thomas, E.J., Biddle, B.J.

Tietz, B.

Thornton, R., Nardi, P.M.

Tosi, H.L.

Treptow, W.

Trommsdorff, V.

Turner, R.H.

Uberla, $\mathrm{K}$.

Voss, H.
Organizations in Action, New York usw. 1967

Basic Concepts for Classifying the Phenomena of Role, in: Role Theory, Concepts and Research, Hrsg.: Biddle, B.J., Thomas, E.J., New York usw. 1966 , S. 23 ff.

The Nature and History of Role Theory, in: Role Theory: Concepts and Research, Hrsg.: B.J. Biddle, E.J. Thomas, New York usw. 1966, S. 3 ff.

Die Haupttendenzen für das Handelsmarketing in den 8oer Jahren, in: Marketing im Wandel, Hrsg.: $\mathrm{H}$. Meffert, Wiesbaden 1980, S. $87 \mathrm{ff}$.

The Dynamics of Role Aquisition, in: AJS 1975, S. 870 ff.

The Effects of Expectation Levels and Role Consensus on the Buyer Seller Dyad, in: JoB 1966, s. 517 ff.

Das Problem der zusätzlichen Dienstleistungen, in: HV + HM 1972, S. $1155 \mathrm{ff}$.

Die Messung von Produktimages für das Marketing. Grundlagen und Operationalisierung, Köln usw. 1975

Role-Taking, role standpoint and reference-group behavior, in: AJS 1956 , S. 316 ff.

Faktorenanalyse, 2. Aufl., Berlin 1971

Art.: Handelsvertreter, in: Management-Enzyklopädie, 3. Band, München 1970 , S. 415 ff. 
Voss, H.

Voss, H.

Voss, $\mathrm{H}$.

Voss, $\mathrm{H}$.

Voss, H. (Hrsg.)

Wallendorf, M. , Zaltmann, G. (Hrsg.)

Weber, A.B.

Webster, F.E., Wind, $Y$.

Webster, F.E., Wind, $Y$.

Wettschureck, G.

Willas, J.
Wandel durch Nachdenken, Handelsvertreter-Praxis, Heft 11, Braunschweig 1978

Handelsvertreter oder Reisende eine Renaissance für Handelsvertreter?, in: Handbuch Marketing Band 2, Hrsg.: J. Koinecke, Gernsbach 1978, S. $671 \mathrm{ff}$.

Handelsvertreter-Marketing, in: Handbuch Marketing, Band 2, Hrsg.: J. Koinecke, Gernsbach 1978, S. $1433 \mathrm{ff}$.

Spezialprobleme des Verkaufs über Handelsvertreter, in: Arbeitshandbuch Absatzförderung, Hrsg.: J. Koinecke, München 1979, S. $295 \mathrm{ff}$.

Handelsvertreter-Handbuch, München 1979

Readings in Consumer Behavior: Individuals, Groups and Organizations, New York usw. 1979

Absatz durch Handelsvertreter oder Reisende, Ludwigshafen 1973

Organizational Buying Behavior, Englewood Cliffs 1972

A General Model for Unterstanding Organizational Buying Behavior, in: JoM 1972, S. 12 ff.

Meßtechnisches Praktikum für Marktforscher, Hamburg 1977

Die Wahl zwischen Handelsvertretern und Reisenden als absatzpolitisches Problem, in: FfH-Mitteilungen, Heft 5, 1975 
Winer, L.,

Schiff, J.S.

Wiswede, G.

Yamane, T.

Zaltman, G. , Burger, P.C.

Zarth, H.R.

Zinser, $w$.
Industrial Salespeople's Views on Motivation, in: IMM 1980, S. $319 \mathrm{ff}$.

Rollentheorie, Stuttgart 1977

Statistik, Frankfurt 1976

Marketing Research, Hinsdale 1975

Setzen Sie Ihre Handelsvertreter erfolgreicher ein, in: MJ 1976, S. $437 \mathrm{ff}$.

Der Absatz von Investitionsgütern Ein Beitrag zur Bestimmung von Marktsegmenten mit Hilfe von Einstellungsdaten, Berlin 1978 



\section{SCHRIFTEN ZUM MARKETING}

Band 1 Friedrich Wehrle: Strategische Marketingplanung in Warenhäusern. Anwendung der Portfolio-Methode. 1981.

Band 2 Jürgen Althans: Die Übertragbarkeit von Werbekonzeptionen auf internationale Märkte. Analyse und Exploration auf der Grundlage einer Befragung bei europaweit tätigen Werbeagenturen. 1982.

Band 3 Günter Kimmeskamp: Die Rollenbeurteilung von Handelsvertretungen. Eine empirische Untersuchung zur Einschätzung des Dienstleistungsangebotes durch Industrie und Handel. 1982.

Band 4 Manfred Bruhn: Konsumentenzufriedenheit und Beschwerden. Erklärungsansätze und Ergebnisse einer empirischen Untersuchung in ausgewählten Konsumbereichen. 1982. 


\author{
KIENAST, ROLF \\ AKTIENANALYSE \\ Möglichkeiten rationaler Anlageentscheidungen
}

Frankfurt/M., Bern, Las Vegas, 1977. $258 \mathrm{~S}$. EUROPÄISCHE HOCHSCHULSCHRIFTEN: Reihe 5, Volks- und Betriebswirtschaft. Bd. 156

ISBN 3-261-02945-5

$$
\text { br. sFr. 43.-- *) }
$$

Der Kauf einer kotierten Aktie stellt den Anleger vor das Entscheidungsproblem, aus einer Vielzahl möglicher Kaufalternativen jene auszuwăhlen, die seinen Nutzenerwartungen am ehesten entspricht. Die Aktienanalyse versucht, för solche Wahlsituationen Entscheidungsgrundlagen zu erarbeiten, um die Aktienanlage einer möglichst rationalen Beurteilung zugänglich zu machen. Die vorliegende Untersuchung bezweckt, die grundsătzlichen Möglichkeiten rationaler Aktienanlageentscheidungen aufzuzeigen und kritisch zu beurteilen.

Aus dem Inhalt: Der Aktienkauf als Entscheidungsproblem - Inhaltliche Deutung der Entscheidungselemente: Anlageziele, Kaufalternativen, relevante Umweltkonstellation für den Anleger, Möglichkeiten der Aktienbewertung.

HOFFMANN, WOLF-DIETRICH

DIE INTERNATIONAL BUSINESS MACHINES CORPORATION (IBM)

IM AMERIKANISCHEN DATENVERARBEITUNGSMARKT -

Eine Fallstudie

Frankfurt/M., Bern, 1975. 300 S. EUROPÄISCHE HOCHSCHULSCHRIFTEN: Reihe 5, Volks- und Betriebswirtschaft. Bd. 112

ISBN 3-261-01848-8

br. sFr. $48.20 *)$

Die Arbeit untersucht die Bestimmungsgründe für IBMs Dominanz im DV-Markt. Die Analyse bedient sich des traditionellen preistheoretischen ebenso wie des neueren verhaltenstheoretischen Instrumentariums. Neben den Determinanten von IBMs Marktmacht (Management: flexible Organisation, Absatzstrategie, Arbeitspolitik/F\&E-Verhalten/Preispolitik/etc.) werden Charakteristika des DV-Marktes aufgezeigt (Struktur von Anbieter und Nachfrager / Typische Verkaufspraktiken wie Leasing, Bündelung etc. / DV-Technologie). Die Arbeit enthălt Beitrăge zur angewandten Markttheorie (Inventions- und Innovationstheorien, organizational slack, Theorien des Unternehmenswachstums, etc.).

Aus dem Inhalt: Konzeption der Studie - Daten zur Entstehung und Entwicklung der IBM und zum DV-Markt - Unternehmensspezifische Einflußfaktoren: Persőnlichkeiten, Organisationsmerkmale, Arbeitspolitik, Absatzstrategie - F\&E-Verhalten (incl. technologische Entwicklung der DV) - Preispolitik - IBM und Antitrust.

*) unverbindliche Preisempfehlung

Auslieferung: Verlag Peter Lang AG, Jupiterstr. 15, CH-3015 Bern 